\title{
Access to Different Isomeric Dibenzoxazepinones through Copper-catalyzed C-H Etherification and C-N Bond Construction with Controllable Smiles Rearrangement
}

Yunfei Zhou, ${ }^{\dagger}$ Jianming Zhu, ${ }^{\dagger}$ Bo Li, ${ }^{*}{ }^{\dagger}$ Yong Zhang, ${ }^{\dagger}$ Jia Feng, ${ }^{\dagger}$ Adrian Hall, ${ }^{\ddagger}$ Jiye Shi, ${ }^{*, \$}$ Weiliang Zhu ${ }^{* \dagger}$

${ }^{\dagger}$ CAS Key Laboratory of Receptor Research, Drug Discovery and Design Center, Shanghai Institute of Materia Medica, Chinese Academy of Sciences, Shanghai, 201203, P. R. China

${ }^{\ddagger}$ UCB Biopharma SPRL, Chemin du Foriest, Braine-l'Alleud, Belgium

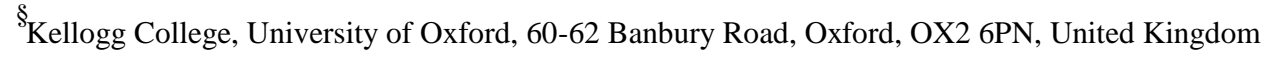

\section{Table of Contents}

(1) General Methods

(2) Optimization of the Reaction Conditions

(3) Experimental Procedures

(4) Analytical Characterization Data of Products

(6) Reference.

(7) Copies of ${ }^{1} \mathrm{H}$ NMR and ${ }^{13} \mathrm{C}$ NMR Spectra for the Products S39 


\section{(1) General Methods}

The reagents (chemicals) were purchased from commercial suppliers, and used without further purification. The reactions were monitored by thin layer chromatography (TLC) of silica gel (HSGF254) at $254 \mathrm{~nm}$ wavelength. All products were characterized by their NMR and MS spectra. ${ }^{1} \mathrm{H}$ and ${ }^{13} \mathrm{C}$ NMR spectra were recorded on a $400 \mathrm{MHz}, 500 \mathrm{MHz}$, or 600 $\mathrm{MHz}$ instrument. Chemical shifts were reported in parts per million (ppm, $\delta$ ) downfield from tetramethylsilane. Proton coupling patterns were described as singlet (s), doublet (d), triplet (t), quartet (q), multiplet (m), doublet of doublets (dd) and broad (br). Low- and high-resolution mass spectra (LRMS and HRMS) were measured on spectrometer. 


\section{(2) Optimization of the Reaction Conditions}

\subsection{Screening of ligands to optimize the non-rearrangement reaction}

Table S1. Optimization of the non-rearrangement reaction conditions with different $\operatorname{ligands}^{a}$

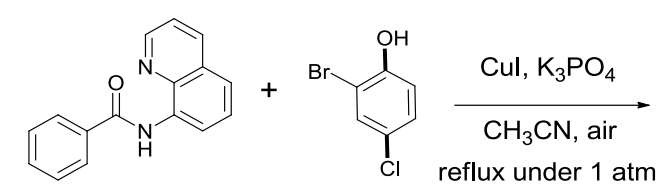

$5 \mathbf{a}$ 6a

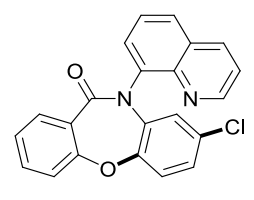

$7 \mathbf{a}$

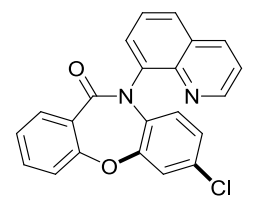

$8 \mathbf{a}$

\begin{tabular}{clcc} 
& & $\begin{array}{c}\text { Non-rearranged } \\
\text { product }\end{array}$ & \multicolumn{2}{c}{$\begin{array}{c}\text { Rearranged } \\
\text { product }\end{array}$} \\
\hline \multirow{2}{*}{ entry } & \multicolumn{1}{c}{ ligands } & \multicolumn{2}{c}{ yield $^{b}(\%)$} \\
\hline $\mathbf{1}$ & - & $\mathbf{7 2}$ & $\mathbf{8 a}$ \\
\hline 2 & HMTA (Hexamethylenetetramine) & 70 & $\mathbf{0}$ \\
3 & DMAP (4-(dimethylamino)-pyridin) & 80 & 0 \\
4 & DABCO (1,4-Diaza[2.2.2]bicyclooctane) & 84 & 0 \\
5 & 2,2'-bipyridine & 80 & 0 \\
6 & pyridine & 78 & 0 \\
7 & {$\left[1,1^{\prime}\right.$-biphenyl]-2,2'-diamine } & 20 & 0 \\
8 & dbm (1,3-diphenylpropane-1,3-dione) & 76 & 0 \\
9 & picolinic acid & 0 & 0 \\
10 & DMEDA (N,N'-Dimethylethylenediamine) & 0 & 0 \\
\hline
\end{tabular}

${ }^{a}$ Reaction conditions: 5 a $(0.2 \mathrm{mmol}), 6 a(0.2 \mathrm{mmol}), \mathrm{CuI}(20 \mathrm{~mol} \%), \mathrm{K}_{3} \mathrm{PO}_{4}(0.6$ mmol), open air, which was reacted at $74{ }^{\circ} \mathrm{C}$ for $1 \mathrm{~h}$ and then refluxed under 1 atm for 4-8 h. ${ }^{b}$ isolated yield.

Based on the previous optimal condition, different ligands were scaned (Table S1). Among these (Table S1, entries 2-10), DABCO gave the best result (Table S1, entry 4). But none of them could improve the yield of $\mathbf{7 a}$ obviously. It is noteworthy that picolinic acid and DMEDA ( $N, N$ '-Dimethyl-1,2-ethanediamine) (Table S1, entry 9, 10), the classic ligands used in amidation reaction, failed to give any products entirely. It indicated that they might capture the copper from the directing group of 8-aminoquinoline interrupting the process of aerobic oxidative $\mathrm{C}-\mathrm{H}$ etherification. Therefore, we didn't use any ligands in the further optimization. 


\subsection{Rearrangement reaction optimization}

As discussed in the controlled experiments, M1 could be transformed into 7a via Goldberg reaction or access to $\mathbf{8 a}$ through a Smiles rearrangement followed by the cyclization. Both of the transformations are in a quantity yield. So if these two regio-isomeric dibenzoxazepinones were accessed in high selectivity, efforts should be paid on choice of the reaction conditions to differentiate the divergent pathways (Scheme S1, Path A2 and Path B2). Based on the optimized non-rearrangement reaction conditions, we want to develop a new way to switch from Path A2 to Path B2. So we begin to scope the reaction conditions to diminish the transformation of M1 into7a.

Scheme S1. Controlled experiments to determine how the temperature and catalyst affecting Path (AB) 1 and Path $\mathrm{A} 2^{a}$

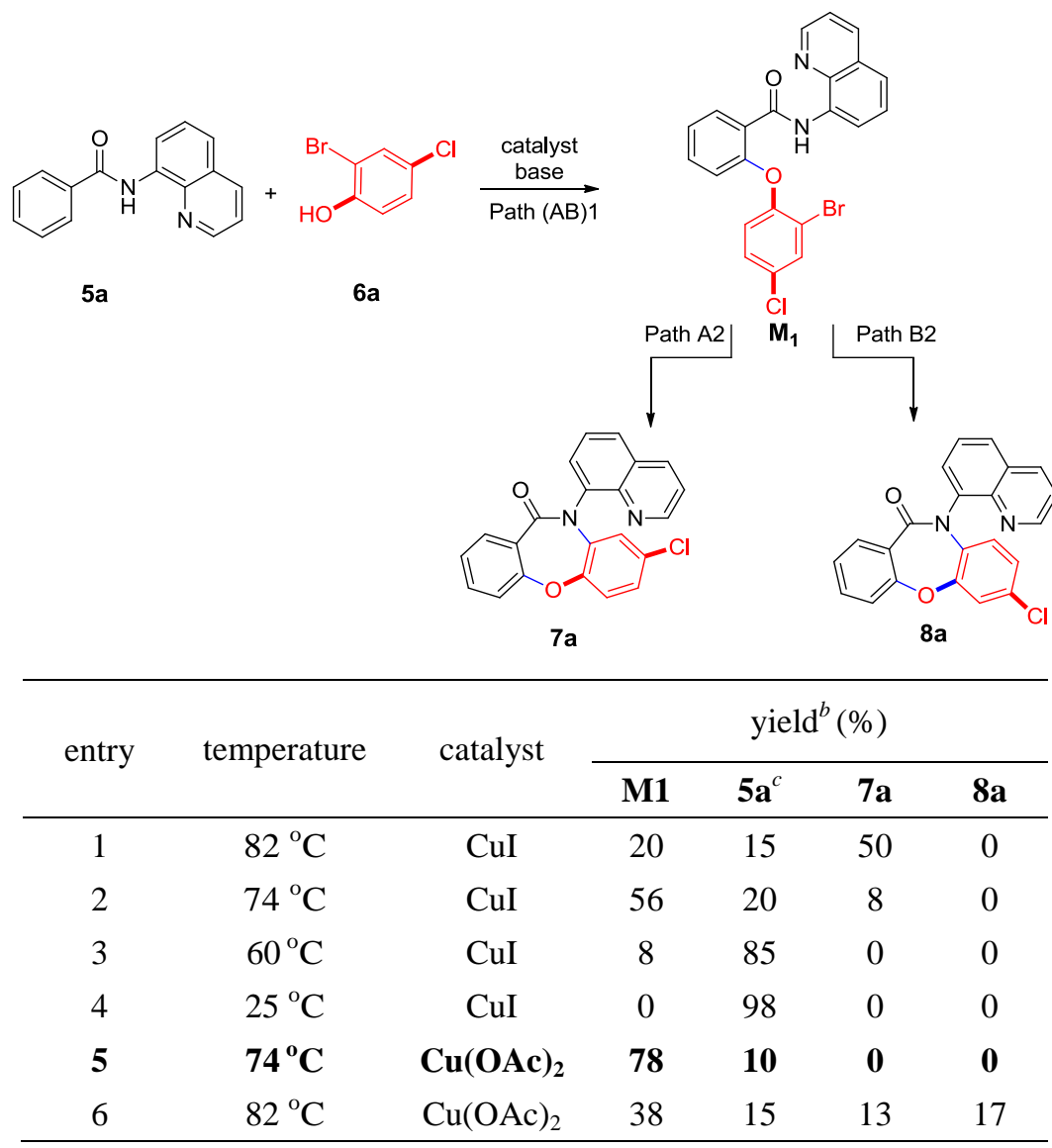

${ }^{a}$ Reaction conditions: $5 \mathbf{a}(0.2 \mathrm{mmol}), \mathbf{6 a}(0.2 \mathrm{mmol})$, copper salts $(20 \mathrm{~mol} \%), \mathrm{K}_{3} \mathrm{PO}_{4}$

(0.4 mmol), open air, $\mathrm{CH}_{3} \mathrm{CN}$ as solvent, 6 h. ${ }^{b}$ isolated yield. ${ }^{c}$ recycled material.

And we found that the temperature played an important role in Path A2. When the 
reaction undergo at $82{ }^{\circ} \mathrm{C}$ using $\mathrm{CuI}$ as the catalyst, 7a is the major product in $50 \%$ yield with $\mathbf{M 1}$ in $20 \%$ yield (Scheme S1, entry 1). As the temperature fell to $74{ }^{\circ} \mathrm{C}$, M1 became the major product in $56 \%$ yield while 7 a reduced to $8 \%$ yield (Scheme S1, entry 2). The reaction could not take place mostly when the temperature fell to $60^{\circ} \mathrm{C}$ or below (Scheme S1, entries $3 \& 4$ ). So $74{ }^{\circ} \mathrm{C}$ is the optimized temperature to guarantee the yield of $\mathbf{M 1}$ and to reduce the yield of $\mathbf{7 a}$. Using $\mathrm{Cu}(\mathrm{II})$ as the catalyst further increased the selectivity of the two pathyways (Scheme S1, entry 5). It suggested that the copper-catalyzed aerobic oxidative $\mathrm{C}-\mathrm{H}$ etherification (Path (AB)1) would be an catalytic circle between $\mathrm{Cu}(\mathrm{II})$ and $\mathrm{Cu}(\mathrm{III})$, meanwhile the copper-catalyzed Goldberg coupling (Path A2) might be an catalytic circle between $\mathrm{Cu}(\mathrm{I})$ and $\mathrm{Cu}(\mathrm{III})$. So a moderate temperature $\left(74^{\circ} \mathrm{C}\right)$ and $\mathrm{Cu}(\mathrm{II})$ guarantee the yield of $\mathbf{M 1}$ (Path (AB)1) and reduce the yield of $\mathbf{7 a}$ (Path A2).

Next step we want to enhance the transformation of M1 into 8a (Path B2). So we screened solvent and base as the followings.

Table S2. Optimization of the rearrangement reaction conditions ${ }^{a}$

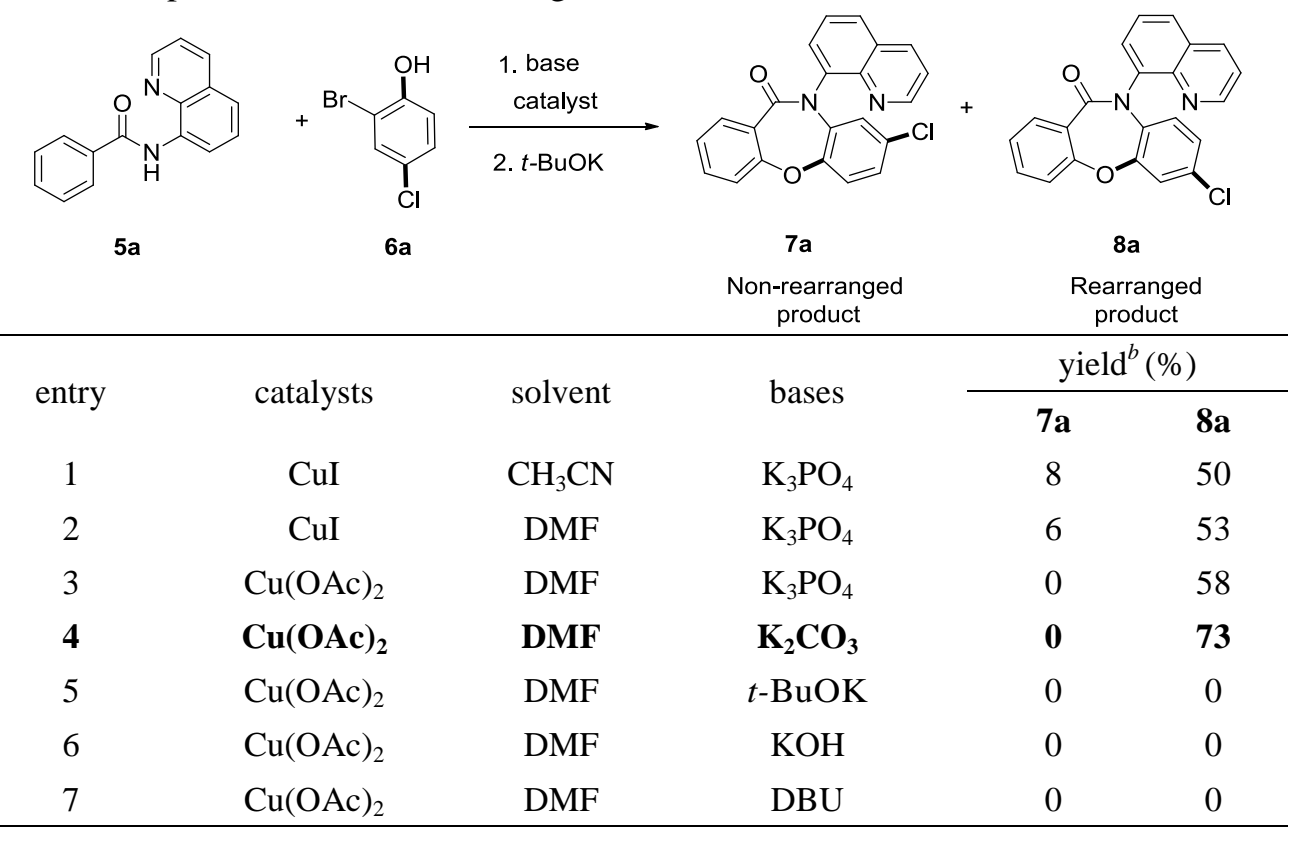

${ }^{a}$ Reaction conditions: $5 \mathbf{a}(0.2 \mathrm{mmol}), \mathbf{6 a}(0.2 \mathrm{mmol})$, copper salts $(20 \mathrm{~mol} \%)$, base $(0.4 \mathrm{mmol})$, open air, $74{ }^{\circ} \mathrm{C}$. After $\mathbf{5 a}$ was consumed entirely, $t$-BuOK $(0.2 \mathrm{mmol})$ was added to the mixture at room temperature. ${ }^{b}$ isolated yield. 
In the process of $\mathrm{C}-\mathrm{H}$ etherification, $\mathrm{CuI}$ was used as catalyst, $\mathrm{K}_{3} \mathrm{PO}_{4}$ as base, and $\mathrm{CH}_{3} \mathrm{CN}$ as solvent. After $\mathbf{5 a}$ was consumed entirely, $t$-BuOK was added to the reaction mixture. Impressively, we found it afforded 7a in $8 \%$ yield and $\mathbf{8 a}$ in $50 \%$ yield (Table S2, entry 1). Changing the solvent $\mathrm{CH}_{3} \mathrm{CN}$ to DMF, reduce the yield of $7 \mathbf{a}$ to $6 \%$ and improve the yield of $8 \mathbf{a}$ to $53 \%$ (Table S2, entry 2). The use of $\mathrm{Cu}(\mathrm{OAc})_{2}$ as catalyst gave 8a exclusively and inhibited 7a completely (Table S2, entry 3). Weak base $\mathrm{K}_{2} \mathrm{CO}_{3}$ gave the best yield (Table S2, entry 4). However, if strong base such as $t$-BuOK, $\mathrm{KOH}$, DBU (1,8-Diazabicyclo[5.4.0]undec-7-ene) was used at the first step, none of products could be detected (Table S2, entry $5,6,7$ ). So the optimized reaction condition involved the use of DMF as solvent, $\mathrm{K}_{2} \mathrm{CO}_{3}$ as base, cupric acetate as catalyst, and after the benzmaides was consumed up, $t$-BuOK was consequently added to the reaction mixture to afford the rearranged product $\mathbf{8 a}$.

\section{(3) Experimental Procedures}

\subsection{General procedure for the synthesis of amides $5^{1}$}

Synthesis of the representative amide $\mathbf{5 a}$.

8-Aminoquinoline (1.86 g, $12.9 \mathrm{mmol})$ was dissolved in dichloromethane $(25 \mathrm{~mL})$, after that, $\mathrm{Et}_{3} \mathrm{~N}(2.4 \mathrm{~mL}, 16.64 \mathrm{mmol})$ was added. The resulting solution was cooled to $0{ }^{\circ} \mathrm{C}$ and then benzoyl chloride ( $2 \mathrm{~g}, 14.23 \mathrm{mmol}$ ) was added dropwise. The resulting mixture was stirred at room temperature overnight. The mixture was quenched by water and extracted with dichloromethane $(50 \mathrm{~mL} \times 3)$. The combined organic layer was dried with $\mathrm{Na}_{2} \mathrm{SO}_{4}$ and concentrated. Purification through column chromatography on silica gel PE:EA (20:1 to 10:1) gave $5 \mathbf{a}(2.8 \mathrm{~g}, 87 \%)$ as a white solid.

\subsection{Copper-catalyzed non-rearrangement reaction between 8 -aminoquinoline benzamides and 2-bromophenols}

The representative reaction of benzamide 5a and 2-bromophenol $6 \mathbf{a}$. 
<smiles>O=C(Nc1cccc2cccnc12)c1ccccc1</smiles>

$5 a$<smiles>Oc1ccc(Cl)cc1Br</smiles>

$6 a$

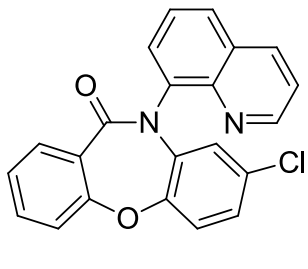

$7 a$

A $10 \mathrm{~mL}$ round-bottom flask was equipped with a magnetic stir bar and charged with $\mathrm{N}$-(quinolin-8-yl)-benzamide (0.100 g, $0.402 \mathrm{mmol}), \mathrm{CuI}(0.015 \mathrm{~g}, 0.080 \mathrm{mmol})$, $\mathrm{K}_{3} \mathrm{PO}_{4}(0.256 \mathrm{~g}, 1.21 \mathrm{mmol}), 2$-bromo-4-chlorophenol $(83.56 \mathrm{mg}, 0.402 \mathrm{mmol})$ and acetonitrile $(5 \mathrm{~mL})$. The flask was then equipped with a reflux condenser, warmed within an oil bath and opened to the air. The resulting mixture was stirred at $74{ }^{\circ} \mathrm{C}$ for $0.5 \mathrm{~h}$ and then refluxed until the full consumption of starting material of the amide, after that, the oil bath was removed. The resulting dark-brown mixture was cooled to room temperature and dissolved in water $(10 \mathrm{~mL})$. The diluted solution was extracted with dichloromethane $(10 \mathrm{~mL} \times 3)$. The combined organic phase was washed with brine (15 mL), dried over sodium sulfate, filtered, and concentrated under reduced pressure. Purification by column chromatography eluting with PE:EA $(6: 1)$ gave 7a $(0.125$ g, $82 \%$ yield) as a yellow solid. $\mathrm{R}_{\mathrm{f}}=0.3\left(\mathrm{SiO}_{2}\right.$, hexanes/ethyl acetate, $\left.3: 1\right)$.

\subsection{Copper-catalyzed rearrangement reaction between 8 -aminoquinoline benzamides} and 2-bromophenols

The representative reaction of benzamide 5a and 2-bromophenol $\mathbf{6 a}$.

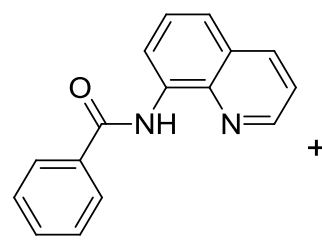

$5 a$

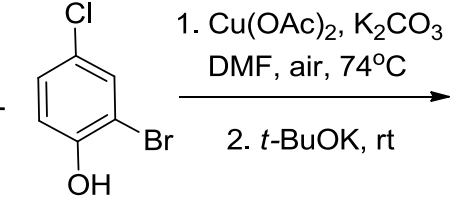

$6 a$<smiles>O=C1c2ccccc2Oc2cc(Cl)ccc2N1c1cccc2cccnc12</smiles>

$8 a$

A $10 \mathrm{~mL}$ round-bottom flask was equipped with a magnetic stir bar and charged with $\mathrm{N}$-(quinolin-8-yl) benzamide $(0.100 \mathrm{~g}, 0.402 \mathrm{mmol}), \mathrm{Cu}(\mathrm{OAc})_{2}(0.014 \mathrm{~g}, 0.08 \mathrm{mmol})$, $\mathrm{K}_{2} \mathrm{CO}_{3}(0.111 \mathrm{~g}, 0.805 \mathrm{mmol})$, 2-bromo-4-chlorophenol (83.56 mg, $\left.0.402 \mathrm{mmol}\right)$ and 
DMF (5 mL). The flask was then equipped with a reflux condenser, placed in an oil bath and opened to the air. The resulting mixture was stirred at $74{ }^{\circ} \mathrm{C}$ monitored by TLC analysis at regular intervals. After the amide starting material was fully consumed (5-10 hours), oil bath was removed. When the mixture was cooled to room temperature, $t$-BuOK $(0.045 \mathrm{~g}, 0.402 \mathrm{mmol})$ was added to the mixture. As the full consumption of M1, the resulting dark-brown mixture was dissolved in water $(10 \mathrm{~mL})$. The diluted solution was extracted with ethyl acetate $(10 \mathrm{~mL} \times 3)$. The organic phase was washed with brine $(15 \mathrm{~mL})$, dried over sodium sulfate, filtered, and concentrated under reduced pressure. Purification by column chromatography eluting with PE:EA (6:1) gave 8a $(0.108 \mathrm{~g}, 73 \%$ yield $)$ as a yellow solid. $\mathrm{R}_{\mathrm{f}}=0.25\left(\mathrm{SiO}_{2}\right.$, hexanes/ethyl acetate, 3:1).

\subsection{Synthesis of the intermediate M1}<smiles>O=C(O)c1ccccc1I</smiles><smiles>Oc1ccc(Cl)cc1Br</smiles>

$6 a$<smiles>O=C(O)c1ccccc1Oc1ccc(Cl)cc1Br</smiles>

10

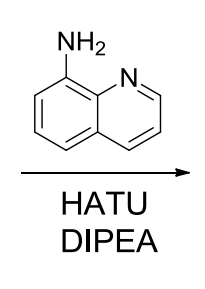<smiles>O=C(Nc1cccc2cccnc12)c1ccccc1Oc1ccc(Cl)cc1Br</smiles>

M1

\subsubsection{Synthesis of $\mathbf{1 0}$}

To a round-bottom flask was added 2-bromo-4-chlorophenol (460mg, $2.22 \mathrm{mmol}$ ), 2-iodobenzoic acid (500 mg, $2.02 \mathrm{mmol})$, CuI (95.99 mg, $0.5 \mathrm{mmol})$, cesium carbonate (1.31g, $4.03 \mathrm{mmol})$ and DMF (10 mL). The flask was purged with nitrogen and the reaction mixture heated to $110{ }^{\circ} \mathrm{C}$ overnight. The reaction was cooled and quenched with $\mathrm{H}_{2} \mathrm{O}$. The diluted solution was extracted with ethyl acetate $(15 \mathrm{~mL} \times 3)$. The organic phase was washed with brine $(30 \mathrm{~mL})$, dried over sodium sulfate, filtered, and concentrated under reduced pressure. Purification by column chromatography eluting with PE:EA (10:1 to1:1) gave 2-(2-bromo-4-chlorophenoxy)benzoic acid (300 mg, 45\%) as a brown solid. 
A solution of 2-(2-bromo-4-chlorophenoxy)benzoic acid (40 mg, $0.12 \mathrm{mmol}$ ), HATU (2-(7-Aza-1H-benzotriazole-1-yl)-1,1,3,3-tetramethyluronium hexafluorophosphate) (93 $\mathrm{mg}, 0.24 \mathrm{mmol}$ ) and DIPEA (Ethyldiisopropylamine) $(31.6 \mathrm{mg}, 0.24 \mathrm{mmol})$ in acetonitrile $(6 \mathrm{~mL})$ was stirred for $15 \mathrm{~min}$ at room temperature. Quinolin-8-amine (18 $\mathrm{mg}, 0.12 \mathrm{mmol}$ ) was added and stirred overnight at room temperature. The reaction was quenched with $\mathrm{H}_{2} \mathrm{O}$. The diluted solution was extracted with ethyl acetate (5 $\mathrm{mL} \times 3)$. The organic phase was washed with brine $(15 \mathrm{~mL})$, dried over sodium sulfate, filtered, and concentrated under reduced pressure. Purification by column chromatography eluting with PE:EA (20:1) gave M1 (25 mg, 45\%) as a yellow solid.

\subsection{Controled experiments about M1 to support the proposed mechanism}

\subsubsection{Synthesis of 7a from M1}

A $10 \mathrm{~mL}$ sealable tube was charged with $\mathbf{M 1}(40 \mathrm{mg}, 0.088 \mathrm{mmol}), \mathrm{K}_{3} \mathrm{PO}_{4}(37.43 \mathrm{mg}$, $0.176 \mathrm{mmol}), \mathrm{CuI}(4.2 \mathrm{mg}, 0.022 \mathrm{mmol})$. Acetonitrile $(5 \mathrm{~mL})$ was added and the tube was sealed. The heterogeneous reaction mixture was heated to $80^{\circ} \mathrm{C}$ for $6 \mathrm{~h}$, cooled to room temperature and dissolved in water $(10 \mathrm{~mL})$. The diluted solution was extracted with dichloromethane $(5 \mathrm{~mL} \times 3)$. The organic phase was washed with brine $(10 \mathrm{~mL})$, dried over sodium sulfate, filtered, and concentrated under reduced pressure. The resulting residue was absorbed with $\mathrm{SiO}_{2}(4 \mathrm{~mL})$ and purified by column chromatography on silica gel (100 mL), eluting with PE:EA (6:1) to obtain $7 \mathbf{a}(31 \mathrm{mg}$, $95 \%)$ as a yellow solid. $\mathrm{R}_{\mathrm{f}}=0.3\left(\mathrm{SiO}_{2}\right.$, hexanes/ethyl acetate, $\left.3: 1\right)$.

\subsubsection{Synthesis of 8a from M1 using $t$-BuOK}

A $10 \mathrm{~mL}$ round-bottom flask was equipped with a magnetic stir bar and charged with M1 (40mg, $0.088 \mathrm{mmol}), t$-BuOK (11.87 mg, $0.105 \mathrm{mmol})$, and DMF (5 mL). The resulting mixture was stirred at room temperature. After the start material was consumed, the mixture was dissolved in water $(10 \mathrm{~mL})$. The diluted solution was extracted with dichloromethane $(10 \mathrm{~mL} \times 3)$. The organic phase was washed with brine $(20 \mathrm{~mL})$, dried over with sodium sulfate, filtered, and concentrated under reduced pressure. The resulting residue was absorbed with $\mathrm{SiO}_{2}(4 \mathrm{~mL})$ purified by column 
chromatography on silica gel $(100 \mathrm{~mL})$, eluting with PE:EA $(6: 1)$ to obtain the pure product $\mathbf{8 a}(31 \mathrm{mg}, 95 \%)$ as a white solid. $\mathrm{R}_{\mathrm{f}}=0.25\left(\mathrm{SiO}_{2}\right.$, hexanes/ethyl acetate, 3:1).

\subsubsection{Preparation of $\mathbf{3}^{2}$}

To a suspension of 2-iodobenzoic acid $(1.24 \mathrm{~g}, 5.00 \mathrm{mmol})$ in DCM $(15 \mathrm{~mL})$ was added DMF (2 drops) followed by oxalyl chloride $(0.530 \mathrm{~mL}, 6.25 \mathrm{mmol})$. The reaction mixture was stirred at $\mathrm{rt}$ for $4 \mathrm{~h}$, and then evaporated to dryness. The acid chloride thus prepared was dissolved in DCM $(15 \mathrm{~mL})$ at $0^{\circ} \mathrm{C}$. Triethylamine $(2.10 \mathrm{~mL}$, $15.0 \mathrm{mmol})$ was added, followed by ethylamine hydrochloride $(0.49 \mathrm{~g}, 6 \mathrm{mmol})$. The reaction mixture was allowed to warm to $\mathrm{rt}$ and stirred for a further $15 \mathrm{~h}$. Water $(50 \mathrm{~mL})$ was added, and the aqueous phase was extracted with DCM $(3 \times 50 \mathrm{~mL})$. The combined organic phase was washed with saturated $\mathrm{Na}_{2} \mathrm{CO}_{3}(20 \mathrm{~mL})$, dried over $\mathrm{Na}_{2} \mathrm{SO}_{4}$, subjected to filtration, and concentrated in vacuo. The crude product was purified by flash chromatography on silica gel, eluting with PE:EA (3:1), to afford 3 (1.0g, 70\% yield).

\subsubsection{Preparation of $\mathbf{M 2}^{2}$}

A sealable tube was charged with copper(I) iodide $(0.033 \mathrm{~g}, 0.173 \mathrm{mmol})$, dibenzoylmethane (0.078 g, $0.346 \mathrm{mmol})$, cesium carbonate $(2.71 \mathrm{~g}, 8.30 \mathrm{mmol})$, N-ethyl-2-iodobenzamide 3 (1.00 g, $3.64 \mathrm{mmol})$ and 2-bromo-4-chlorophenol (0.861 g, $4.15 \mathrm{mmol})$. Toluene $(15 \mathrm{~mL})$ was added and the tube was sealed. The heterogeneous reaction mixture was heated to $120{ }^{\circ} \mathrm{C}$ for $6 \mathrm{~h}$, cooled to room temperature and partitioned between $2 \mathrm{M} \mathrm{HCl}(30 \mathrm{~mL})$ and EtOAc $(3 \times 30 \mathrm{~mL})$. The combined organics were washed with saturated brine $(30 \mathrm{~mL})$, dried over $\mathrm{Na}_{2} \mathrm{SO}_{4}$, subjected to filtration, and concentrated in vacuo. The crude product was purified by flash chromatography on silica gel, eluting with PE:EA (9:1 to 2:1), to deliver the title compound M2 as a colourless oil $(0.788 \mathrm{~g}, 62 \%)$.

\subsubsection{Synthesis of $\mathbf{4}$ from $\mathbf{M} \mathbf{2}^{2}$}

A sealable tube $(10 \mathrm{~mL})$ was charged with tripotassium phosphate $(0.25 \mathrm{~g}, 1.2 \mathrm{mmol})$ 
and M2 $(0.34 \mathrm{~g}, 1 \mathrm{mmol})$. DMF $(5 \mathrm{~mL})$ was added with the tube sealed. The heterogeneous reaction mixture was heated to $120{ }^{\circ} \mathrm{C}$ for $6 \mathrm{~h}$, cooled to $\mathrm{rt}$ and partitioned between water $(25 \mathrm{~mL})$ and EtOAc $(3 \times 25 \mathrm{~mL})$. The combined organic phase was washed with saturated brine $(25 \mathrm{~mL})$, dried over $\mathrm{Na}_{2} \mathrm{SO}_{4}$, subjected to filtration, and concentrated in vacuo. The crude product was purified by flash chromatography on silica gel, eluting with PE:EA (10:1 to $3: 1)$ to deliver the title compound 4 as a yellow oil (0.26g, $95 \%$ yield).

\subsection{Removal of the directing group ${ }^{3}$}

The representative transformation from 7lc into $9 \mathbf{c}$.

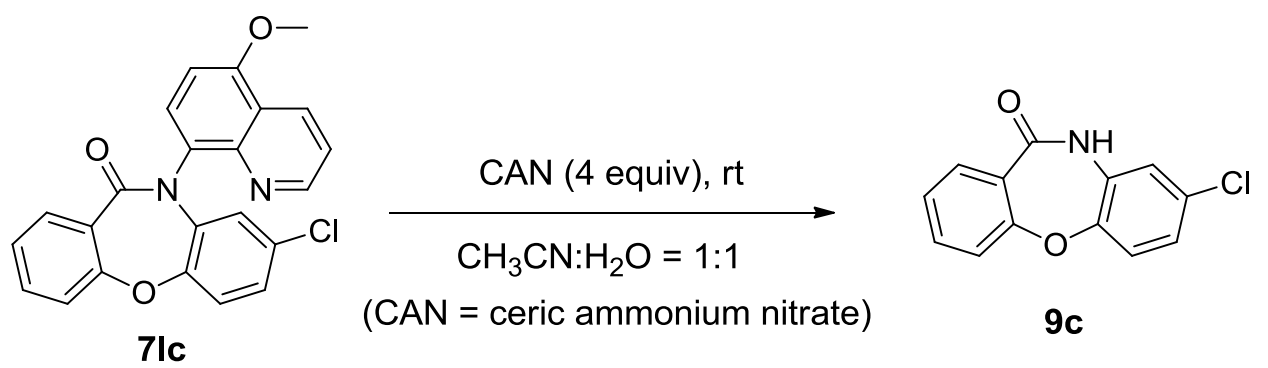

A $10 \mathrm{~mL}$ round-bottom flask was equipped with a magnetic stir bar and charged with 7lc $(35 \mathrm{mg}, 0.08 \mathrm{mmol})$ in $\mathrm{MeCN}(2.5 \mathrm{~mL})$ at RT. A solution of CAN (ceric ammonium nitrate) (175 mg, $0.32 \mathrm{mmol}, 4$ equiv) in $\mathrm{H}_{2} \mathrm{O}(2.5 \mathrm{~mL})$ was added to the substance above. The resulting solution was stirred at $\mathrm{rt}$ for $16 \mathrm{~h} . \mathrm{H}_{2} \mathrm{O}(15 \mathrm{~mL})$ was added and the reaction mixture was extracted with EtOAc $(3 \times 15 \mathrm{~mL})$. Combined organic phase was washed with $10 \%$ $\mathrm{Na}_{2} \mathrm{SO}_{3}$ solution $(2 \times 15 \mathrm{~mL})$ and brine $(15 \mathrm{~mL})$, dried over $\mathrm{Na}_{2} \mathrm{SO}_{4}$, filtered, and evaporated. After column chromatography PE:EA (5:1) $11 \mathrm{mg}(50 \%)$ of $9 \mathrm{c}$ was obtained. $\mathrm{R}_{\mathrm{f}}=0.3\left(\mathrm{SiO}_{2}\right.$, hexanes/ethyl acetate, $3: 1)$. 


\section{(4) Analytical Characterization Data of Products}<smiles>O=C1c2ccccc2Oc2ccc(Cl)cc2N1c1cccc2cccnc12</smiles>

$7 \mathbf{a}$

\section{8-chloro-10-(quinolin-8-yl)dibenzo[b,f][1,4]oxazepin-11(10H)-one 7a}

7a was obtained as a yellow solid by column chromatography (PE/EA $=10 / 1$ to $5 / 1$ ), yield $82 \%(61 \mathrm{mg})$.

${ }^{1} \mathrm{H}$ NMR $\left(400 \mathrm{MHz}, \mathrm{CDCl}_{3}\right) \delta_{\mathrm{H}} 8.96(1 \mathrm{H}, \mathrm{dd}, J=4.2,1.7 \mathrm{~Hz}), 8.27(1 \mathrm{H}, \mathrm{dd}, \mathrm{J}=8.3,1.7$ Hz), $7.99-7.90(2 \mathrm{H}, \mathrm{m}), 7.79$ (1 H, dd, $J=7.3,1.4 \mathrm{~Hz}), 7.66(1 \mathrm{H}, \mathrm{dd}, J=8.2,7.3 \mathrm{~Hz})$, $7.55-7.44(2 \mathrm{H}, \mathrm{m}), 7.31-7.22(3 \mathrm{H}, \mathrm{m}), 7.01(1 \mathrm{H}, \mathrm{dd}, J=8.6,2.5 \mathrm{~Hz}), 6.60(1 \mathrm{H}, \mathrm{d}, J$ $=2.4 \mathrm{~Hz}) .{ }^{13} \mathrm{C}$ NMR $\left(125 \mathrm{MHz}, \mathrm{CDCl}_{3}\right) \delta_{\mathrm{C}} 166.5,160.2,151.8,151.5,144.7,139.4$, $137.0,136.5,133.8,132.5,130.5,130.1,129.5,129.2,126.9,126.8,125.8,125.6,124.1$, 122.5, 122.0, 119.8. LRMS (ESI) $[\mathrm{M}+\mathrm{H}]^{+}$found m/z 373.2. HRMS (ESI) $[\mathrm{M}+\mathrm{H}]^{+}$found $\mathrm{m} / \mathrm{z} 373.0734$, calcd for $\mathrm{C}_{22} \mathrm{H}_{14} \mathrm{ClN}_{2} \mathrm{O}_{2} 373.0744$.

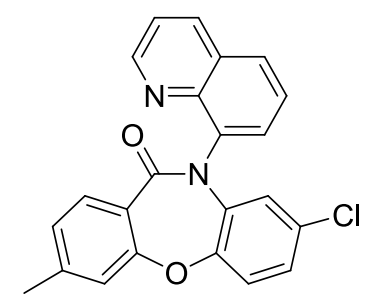

7b

\section{8-chloro-3-methyl-10-(quinolin-8-yl)dibenzo[b,f][1,4]oxazepin-11(10H)-one 7b}

7b was obtained as a yellow solid purified by column chromatography $(\mathrm{PE} / \mathrm{EA}=10 / 1$ to 5/1), $70 \%$ yield (54 mg).

${ }^{1} \mathrm{H}$ NMR $\left(400 \mathrm{MHz}, \mathrm{CDCl}_{3}\right) \delta_{\mathrm{H}} 8.98(1 \mathrm{H}, \mathrm{dd}, J=4.2,1.7 \mathrm{~Hz}), 8.24(1 \mathrm{H}, \mathrm{dd}, J=8.3,1.7$ Hz), $7.97-7.86(2 \mathrm{H}, \mathrm{m}), 7.82(1 \mathrm{H}, \mathrm{dd}, J=7.3,1.4 \mathrm{~Hz}), 7.66(1 \mathrm{H}, \mathrm{dd}, J=8.2,7.3 \mathrm{~Hz})$, $7.46(1 \mathrm{H}, \mathrm{dd}, J=8.3,4.2 \mathrm{~Hz}), 7.34-7.22(1 \mathrm{H}, \mathrm{m}), 7.11(2 \mathrm{H}, \mathrm{d}, J=7.8 \mathrm{~Hz}), 7.03(1 \mathrm{H}$, $\mathrm{dd}, J=8.6,2.5 \mathrm{~Hz}), 6.67(1 \mathrm{H}, \mathrm{d}, J=2.5 \mathrm{~Hz}) .{ }^{13} \mathrm{C} \mathrm{NMR}\left(125 \mathrm{MHz}, \mathrm{CDCl}_{3}\right) \delta_{\mathrm{C}} 166.0$, 
$159.6,151.3,151.0,144.5,144.3,139.0,136.6,136.0,131.8,129.9,129.7,129.0,128.7$, $126.3,126.0,125.2,123.6,123.4,122.1,121.5,119.6,21.0$. LRMS (ESI) $[\mathrm{M}+\mathrm{H}]^{+}$found $\mathrm{m} / \mathrm{z}$ 387.3. HRMS (ESI) $[\mathrm{M}+\mathrm{H}]^{+}$found $\mathrm{m} / \mathrm{z} 387.0884$, calcd for $\mathrm{C}_{23} \mathrm{H}_{16} \mathrm{ClN}_{2} \mathrm{O}_{2} 387.0895$.

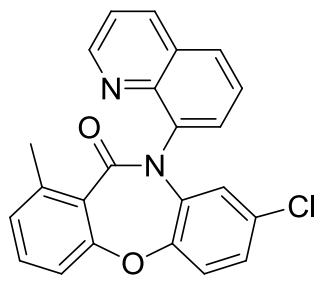

7c

\section{8-chloro-1-methyl-10-(quinolin-8-yl)dibenzo[b,f][1,4]oxazepin-11(10H)-one 7c}

7c was obtained as a white solid through column chromatography $(\mathrm{PE} / \mathrm{EA}=10 / 1$ to $5 / 1)$, yield $62 \%$ (48 mg).

${ }^{1} \mathrm{H}$ NMR $\left(600 \mathrm{MHz}, \mathrm{CDCl}_{3}\right) \delta_{\mathrm{H}} 9.03(1 \mathrm{H}, \mathrm{d}, J=4.7 \mathrm{~Hz}), 8.34(1 \mathrm{H}, \mathrm{d}, J=7.9 \mathrm{~Hz}), 7.99$ $(1 \mathrm{H}, \mathrm{dd}, J=8.1,1.4 \mathrm{~Hz}), 7.84-7.64(2 \mathrm{H}, \mathrm{m}), 7.57(1 \mathrm{H}, \mathrm{dd}, J=7.8,4.2 \mathrm{~Hz}), 7.36(1 \mathrm{H}$, $\mathrm{t}, J=7.8 \mathrm{~Hz}), 7.28(1 \mathrm{H}, \mathrm{d}, J=8.6 \mathrm{~Hz}), 7.15(2 \mathrm{H}, \mathrm{t}, J=7.4 \mathrm{~Hz}), 7.04(1 \mathrm{H}, \mathrm{dd}, J=8.7$, $2.5 \mathrm{~Hz}), 6.49(1 \mathrm{H}, \mathrm{s}), 2.61(3 \mathrm{H}, \mathrm{s}) .{ }^{13} \mathrm{C} \mathrm{NMR}\left(150 \mathrm{MHz}, \mathrm{CDCl}_{3}\right) \delta_{\mathrm{C}} 167.2,161.0,152.2$, $151.4,144.5,142.2,140.0,137.3,136.7,131.7,130.6,129.9,129.4,129.1,128.5,127.2$, 126.5, 125.4, 123.7, 122.3, 122.2, 117.0, 20.5. LRMS (ESI) $[\mathrm{M}+\mathrm{H}]^{+}$found $\mathrm{m} / \mathrm{z} 387.3$. HRMS (ESI) $[\mathrm{M}+\mathrm{Na}]^{+}$found $\mathrm{m} / \mathrm{z}$ 409.0705, calcd. for $\mathrm{C}_{23} \mathrm{H}_{15} \mathrm{O}_{2} \mathrm{~N}_{2} \mathrm{ClNa} 409.0714$.

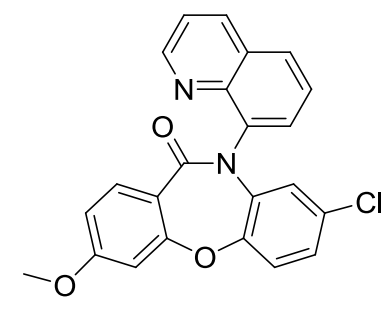

$7 d$

\section{8-chloro-3-methoxy-10-(quinolin-8-yl)dibenzo[b,f][1,4]oxazepin-11(10H)-one 7d}

7d was obtained as a yellow solid through column chromatography (PE/EA $=10 / 1$ to 3/1), yield 57\% (47 mg).

${ }^{1} \mathrm{H}$ NMR $\left(400 \mathrm{MHz}, \mathrm{CDCl}_{3}\right) \delta_{\mathrm{H}} 8.98(1 \mathrm{H}, \mathrm{dd}, J=4.2,1.7 \mathrm{~Hz}), 8.29(1 \mathrm{H}, \mathrm{dd}, J=8.4,1.7$ Hz), $7.97(1 \mathrm{H}, \mathrm{dd}, J=8.2,1.4 \mathrm{~Hz}), 7.90(1 \mathrm{H}, \mathrm{d}, J=8.6 \mathrm{~Hz}), 7.81(1 \mathrm{H}, \mathrm{dd}, J=7.4,1.4$ 
Hz), $7.68(1 \mathrm{H}, \mathrm{dd}, J=8.1,7.4 \mathrm{~Hz}), 7.51(1 \mathrm{H}, \mathrm{dd}, J=8.3,4.2 \mathrm{~Hz}), 7.27(1 \mathrm{H}, \mathrm{d}, J=8.7$ Hz), $7.04(1 \mathrm{H}, \mathrm{dd}, J=8.6,2.5 \mathrm{~Hz}), 6.83(1 \mathrm{H}, \mathrm{dd}, J=8.7,2.5 \mathrm{~Hz}), 6.79(1 \mathrm{H}, \mathrm{d}, J=2.4$ $\mathrm{Hz}), 6.63(1 \mathrm{H}, \mathrm{d}, J=2.5 \mathrm{~Hz}), 3.90(3 \mathrm{H}, \mathrm{s}) .{ }^{13} \mathrm{C} \mathrm{NMR}\left(125 \mathrm{MHz}, \mathrm{CDCl}_{3}\right) \delta_{\mathrm{C}} 166.1,164.1$, $161.5,151.5,151.4,144.9,139.4,137.1,136.4,133.8,130.5,130.2,129.5,129.2,126.8$, 125.6, 124.1, 122.5, 121.9, 118.9, 111.7, 104.7, 55.7. LRMS (ESI) $[\mathrm{M}+\mathrm{H}]^{+}$found $\mathrm{m} / \mathrm{z} 403.3$. HRMS (ESI) $[\mathrm{M}+\mathrm{H}]^{+}$found $\mathrm{m} / \mathrm{z}$ 403.0833, calcd for $\mathrm{C}_{23} \mathrm{H}_{16} \mathrm{O}_{3} \mathrm{~N}_{2} \mathrm{Cl} 403.0844$.

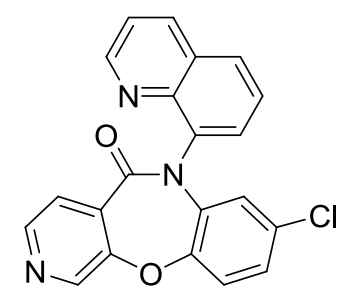

$7 e$

\section{8-chloro-6-(quinolin-8-yl)benzo[b]pyrido[4,3-f][1,4]oxazepin-5(6H)-one 7e}

7e was obtained as a yellow solid through column chromatography (PE/EA $=6 / 1$ to $3 / 1$ ), yield $79 \%$ (60 mg).

${ }^{1} \mathrm{H}$ NMR $\left(400 \mathrm{MHz}, \mathrm{CDCl}_{3}\right) \delta_{\mathrm{H}} 8.97(1 \mathrm{H}, \mathrm{dd}, J=4.2,1.7 \mathrm{~Hz}), 8.71(1 \mathrm{H}, \mathrm{d}, J=0.7 \mathrm{~Hz})$, $8.60(1 \mathrm{H}, \mathrm{d}, J=4.9 \mathrm{~Hz}), 8.30(1 \mathrm{H}, \mathrm{dd}, J=8.4,1.7 \mathrm{~Hz}), 8.00(1 \mathrm{H}, \mathrm{dd}, J=8.3,1.4 \mathrm{~Hz})$, 7.88 - $7.77(2 \mathrm{H}, \mathrm{m}), 7.69(1 \mathrm{H}, \mathrm{dd}, J=8.2,7.3 \mathrm{~Hz}), 7.53(1 \mathrm{H}, \mathrm{dd}, J=8.3,4.2 \mathrm{~Hz}), 7.34$ $(1 \mathrm{H}, \mathrm{d}, J=8.7 \mathrm{~Hz}), 7.09(1 \mathrm{H}, \mathrm{dd}, J=8.7,2.5 \mathrm{~Hz}), 6.65(1 \mathrm{H}, \mathrm{d}, J=2.4 \mathrm{~Hz}) .{ }^{13} \mathrm{C} \mathrm{NMR}$ $\left(125 \mathrm{MHz}, \mathrm{CDCl}_{3}\right) \delta_{\mathrm{C}} 164.5,155.4,151.5,151.5,146.9,144.2,142.3,138.6,136.4$, 136.0, 133.2, 131.0, 129.8, 129.4, 129.3, 126.7, 126.2, 124.8, 124.4, 122.5, 122.0. LRMS (ESI) $[\mathrm{M}+\mathrm{H}]^{+}$found $\mathrm{m} / \mathrm{z}$ 374.2. HRMS (ESI) $[\mathrm{M}+\mathrm{H}]^{+}$found $\mathrm{m} / \mathrm{z}$ 374.0690, calcd for $\mathrm{C}_{21} \mathrm{H}_{13} \mathrm{~N}_{3} \mathrm{O}_{2} \mathrm{Cl} 374.0691$.<smiles>O=C1c2cc(C(F)(F)F)ccc2Oc2ccc(Cl)cc2N1c1cccc2cccnc12</smiles>

$7 f$

8-chloro-10-(quinolin-8-yl)-2-(trifluoromethyl)dibenzo[b,f][1,4]oxazepin-11(10H)-on 
e $7 \mathbf{f}$

7f was obtained as a yellow solid through column chromatography (PE/EA $=10 / 1$ to 5/1), yield $85 \%$ (75 mg).

${ }^{1} \mathrm{H}$ NMR $\left(400 \mathrm{MHz}, \mathrm{CDCl}_{3}\right) \delta_{\mathrm{H}} 8.99(1 \mathrm{H}, \mathrm{dd}, J=4.2,1.7 \mathrm{~Hz}), 8.32(1 \mathrm{H}, \mathrm{d}, J=1.7 \mathrm{~Hz})$, $8.29(1 \mathrm{H}, \mathrm{d}, J=2.4 \mathrm{~Hz}), 8.00(1 \mathrm{H}, \mathrm{dd}, J=8.2,1.4 \mathrm{~Hz}), 7.85-7.77(2 \mathrm{H}, \mathrm{m}), 7.70(1 \mathrm{H}$, $\mathrm{dd}, J=8.2,7.3 \mathrm{~Hz}), 7.53(1 \mathrm{H}, \mathrm{dd}, J=8.3,4.2 \mathrm{~Hz}), 7.42(1 \mathrm{H}, \mathrm{d}, J=8.5 \mathrm{~Hz}), 7.30(1 \mathrm{H}, \mathrm{d}$, $J=8.7 \mathrm{~Hz}), 7.07(1 \mathrm{H}, \mathrm{dd}, J=8.7,2.5 \mathrm{~Hz}), 6.65(1 \mathrm{H}, \mathrm{d}, J=2.4 \mathrm{~Hz}) \cdot{ }^{13} \mathrm{C}$ NMR $(125$ $\left.\mathrm{MHz}, \mathrm{CDCl}_{3}\right) \delta_{\mathrm{C}} 165.2,162.3,151.6,151.1,144.5,138.9,136.5,131.1,130.6\left(\mathrm{q},{ }^{3} J_{\mathrm{C}-\mathrm{F}}=\right.$ $3.5 \mathrm{~Hz}), 130.3\left(\mathrm{q},{ }^{3} J_{\mathrm{C}-\mathrm{F}}=3.9 \mathrm{~Hz}\right), 130.0,129.5,128.2\left(\mathrm{q},{ }^{2} J_{\mathrm{C}-\mathrm{F}}=33.6 \mathrm{~Hz}\right), 127.5,126.8$, $126.1,124.6,123.5\left(\mathrm{q},{ }^{1} J_{\mathrm{C}-\mathrm{F}}=272.4 \mathrm{~Hz}\right), 122.6,122.1,120.7$. LRMS $(\mathrm{ESI})[\mathrm{M}+\mathrm{H}]^{+}$ found $\mathrm{m} / \mathrm{z}$ 441.1. HRMS (ESI) $[\mathrm{M}+\mathrm{H}]^{+}$found $\mathrm{m} / \mathrm{z} 441.0618$, calcd for $\mathrm{C}_{23} \mathrm{H}_{13} \mathrm{O}_{2} \mathrm{~N}_{2} \mathrm{ClF}_{3}$ 441.0612.

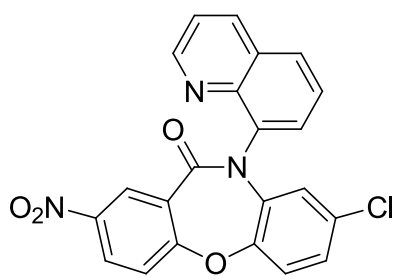

$7 g$

\section{8-chloro-2-nitro-10-(quinolin-8-yl)dibenzo[b,f][1,4]oxazepin-11(10H)-one $7 \mathrm{~g}$}

7 g was obtained as a brown solid through column chromatography (PE/EA = 10/1 to 4/1), yield $79 \%(67 \mathrm{mg})$.

${ }^{1} \mathrm{H}$ NMR $\left(400 \mathrm{MHz}, \mathrm{CDCl}_{3}\right) \delta_{\mathrm{H}} 9.00(1 \mathrm{H}, \mathrm{dd}, \mathrm{J}=4.2,1.7 \mathrm{~Hz}), 8.88(1 \mathrm{H}, \mathrm{d}, J=2.8 \mathrm{~Hz})$, $8.40(1 \mathrm{H}, \mathrm{dd}, J=8.9,2.8 \mathrm{~Hz}), 8.02(1 \mathrm{H}, \mathrm{dd}, J=8.3,1.4 \mathrm{~Hz}), 7.82(1 \mathrm{H}, \mathrm{dd}, J=7.3,1.4$ Hz), $7.71(1 \mathrm{H}$, dd, $J=8.2,7.3 \mathrm{~Hz}), 7.56(1 \mathrm{H}, \mathrm{dd}, J=8.3,4.2 \mathrm{~Hz}), 7.45(1 \mathrm{H}, \mathrm{d}, J=8.9$ $\mathrm{Hz}), 7.34-7.27(2 \mathrm{H}, \mathrm{m}), 7.09(1 \mathrm{H}, \mathrm{dd}, J=8.6,2.4 \mathrm{~Hz}), 6.65(1 \mathrm{H}, \mathrm{d}, J=2.4 \mathrm{~Hz}) .{ }^{13} \mathrm{C}$ $\operatorname{NMR}\left(125 \mathrm{MHz}, \mathrm{CDCl}_{3}\right) \delta_{\mathrm{C}} 163.8,163.7,151.2,150.2,144.8,143.9,138.1,136.1,135.8$, 131.0, 129.4, 129.2, 129.0, 128.4, 128.1, 127.6, 126.3, 125.8, 124.0, 122.1, 121.7, 120.7. LRMS (ESI) $[\mathrm{M}+\mathrm{H}]^{+}$found $\mathrm{m} / \mathrm{z}$ 418.2. HRMS (ESI) $[\mathrm{M}+\mathrm{H}]^{+}$found $\mathrm{m} / \mathrm{z}$ 418.0579, calcd for $\mathrm{C}_{22} \mathrm{H}_{13} \mathrm{O}_{4} \mathrm{~N}_{3} \mathrm{Cl} 418.0589$. 
<smiles>O=C1c2ccc(OC(F)(F)F)cc2Oc2ccc(Cl)cc2N1c1cccc2cccnc12</smiles>

$7 h$

\section{8-chloro-10-(quinolin-8-yl)-3-(trifluoromethoxy)dibenzo[b,f] $[1,4]$ oxazepin-11(10H)-o} ne $7 \mathrm{~h}$

7h was obtained as a yellow solid through column chromatography $(\mathrm{PE} / \mathrm{EA}=10 / 1$ to 3/1), yield $75 \%(67 \mathrm{mg})$.

${ }^{1} \mathrm{H}$ NMR $\left(400 \mathrm{MHz}, \mathrm{CDCl}_{3}\right) \delta_{\mathrm{H}} 8.98(1 \mathrm{H}, \mathrm{dd}, J=4.2,1.7 \mathrm{~Hz}), 8.30(1 \mathrm{H}, \mathrm{dd}, J=8.3,1.7$ Hz), $8.08-7.93(2 \mathrm{H}, \mathrm{m}), 7.81(1 \mathrm{H}, \mathrm{dd}, J=7.3,1.4 \mathrm{~Hz}), 7.69(1 \mathrm{H}, \mathrm{dd}, J=8.2,7.3 \mathrm{~Hz})$, $7.52(1 \mathrm{H}, \mathrm{dd}, J=8.3,4.2 \mathrm{~Hz}), 7.31(1 \mathrm{H}, \mathrm{s}), 7.20-7.13(2 \mathrm{H}, \mathrm{m}), 7.07(1 \mathrm{H}, \mathrm{dd}, J=8.6$, $2.4 \mathrm{~Hz}), 6.64(1 \mathrm{H}, \mathrm{d}, J=2.4 \mathrm{~Hz}) .{ }^{13} \mathrm{C} \mathrm{NMR}\left(125 \mathrm{MHz}, \mathrm{CDCl}_{3}\right) \delta_{\mathrm{C}} 164.9,160.3,152.2$, $152.2,151.1,150.8,144.2,138.6,136.1,136.1,133.6,130.6,129.6,129.1,129.0,126.4$ 125.6, 124.8, 123.8, 122.1, $121.8\left(\mathrm{q},{ }^{1} J_{\mathrm{C}-\mathrm{F}}=259.3 \mathrm{~Hz}\right), 121.6,111.7$. LRMS (ESI) $[\mathrm{M}+\mathrm{H}]^{+}$found $\mathrm{m} / \mathrm{z}$ 457.2. HRMS (ESI) $[\mathrm{M}+\mathrm{H}]^{+}$found $\mathrm{m} / \mathrm{z}$ 457.0566, calcd for $\mathrm{C}_{23} \mathrm{H}_{13} \mathrm{O}_{3} \mathrm{~N}_{2} \mathrm{ClF}_{3} 457.0561$.<smiles>O=C1c2ccc(F)cc2Oc2ccc(Cl)cc2N1c1cccc2cccnc12</smiles>

$7 i$

\section{8-chloro-3-fluoro-10-(quinolin-8-yl)dibenzo[b,f][1,4]oxazepin-11(10H)-one $7 \mathrm{i}$}

7i was obtained as a white solid through column chromatography (PE/EA = 10/1 to 5/1), yield $83 \%$ (65 mg).

${ }^{1} \mathrm{H} \mathrm{NMR}\left(400 \mathrm{MHz}, \mathrm{CDCl}_{3}\right) \delta_{\mathrm{H}} 8.98(1 \mathrm{H}, \mathrm{dd}, J=4.2,1.6 \mathrm{~Hz}), 8.30(1 \mathrm{H}, \mathrm{dd}, J=8.4,1.7$ Hz), $8.02-7.92(2 \mathrm{H}, \mathrm{m}), 7.81(1 \mathrm{H}, \mathrm{dd}, J=7.4,1.3 \mathrm{~Hz}), 7.69(1 \mathrm{H}, \mathrm{dd}, J=8.2,7.3 \mathrm{~Hz})$, $7.52(1 \mathrm{H}, \mathrm{dd}, J=8.3,4.2 \mathrm{~Hz}), 7.29(1 \mathrm{H}, \mathrm{d}, J=0.7 \mathrm{~Hz}), 7.12-6.97(3 \mathrm{H}, \mathrm{m}), 6.64(1 \mathrm{H}$, $\mathrm{d}, J=2.5 \mathrm{~Hz}) .{ }^{13} \mathrm{C}$ NMR $(125 \mathrm{MHz}$, Acetone $) \delta_{\mathrm{C}} 165.4\left(\mathrm{~d},{ }^{1} J_{\mathrm{C}-\mathrm{F}}=253.0 \mathrm{~Hz}\right), 164.7,161.2$ 
$\left(\mathrm{d},{ }^{3} J_{\mathrm{C}-\mathrm{F}}=11.6 \mathrm{~Hz}\right), 151.5,151.3,144.6,139.2,137.2,136.5,134.2\left(\mathrm{~d},{ }^{3} J_{\mathrm{C}-\mathrm{F}}=10.5 \mathrm{~Hz}\right)$, $130.6,130.2,129.6,129.3,126.7,125.6,123.7,123.6\left(\mathrm{~d},{ }^{4} J_{\mathrm{C}-\mathrm{F}}=3.5 \mathrm{~Hz}\right), 123.0,122.1$, $113.1\left(\mathrm{~d},{ }^{2} J_{\mathrm{C}-\mathrm{F}}=22.0 \mathrm{~Hz}\right), 107.5\left(\mathrm{~d},{ }^{2} J_{\mathrm{C}-\mathrm{F}}=24.1 \mathrm{~Hz}\right)$. LRMS $(\mathrm{ESI})[\mathrm{M}+\mathrm{H}]^{+}$found $\mathrm{m} / \mathrm{z}$ 391.4. HRMS (ESI) $[\mathrm{M}+\mathrm{H}]^{+}$found $\mathrm{m} / \mathrm{z} 391.0633$, calcd for $\mathrm{C}_{22} \mathrm{H}_{13} \mathrm{O}_{2} \mathrm{~N}_{2} \mathrm{ClF} 391.0644$.<smiles>O=C1c2cc(Cl)ccc2Oc2ccc(Cl)cc2N1c1cccc2cccnc12</smiles>

$7 \mathrm{j}$

\section{2,8-dichloro-10-(quinolin-8-yl)dibenzo[b,f][1,4]oxazepin-11(10H)-one 7j}

$7 \mathbf{j}$ was obtained as a yellow solid through column chromatography (PE/EA = 10/1 to 5/1), yield $73 \%$ (59 mg).

${ }^{1} \mathrm{H}$ NMR $\left(400 \mathrm{MHz}, \mathrm{CDCl}_{3}\right) \delta_{\mathrm{H}} 8.98(1 \mathrm{H}, \mathrm{dd}, J=4.2,1.7 \mathrm{~Hz}), 8.30(1 \mathrm{H}, \mathrm{dd}, J=8.3,1.7$ Hz), $7.99(1 \mathrm{H}, \mathrm{dd}, J=8.2,1.4 \mathrm{~Hz}), 7.93(1 \mathrm{H}, \mathrm{d}, J=2.7 \mathrm{~Hz}), 7.80(1 \mathrm{H}, \mathrm{dd}, J=7.4,1.4$ Hz), $7.69(1 \mathrm{H}, \mathrm{dd}, J=8.2,7.3 \mathrm{~Hz}), 7.53(1 \mathrm{H}, \mathrm{dd}, J=8.3,4.2 \mathrm{~Hz}), 7.48(1 \mathrm{H}, \mathrm{dd}, J=8.6$, $2.7 \mathrm{~Hz}), 7.30-7.22(2 \mathrm{H}, \mathrm{m}), 7.06(1 \mathrm{H}, \mathrm{dd}, J=8.6,2.5 \mathrm{~Hz}), 6.62(1 \mathrm{H}, \mathrm{d}, J=2.4 \mathrm{~Hz})$. ${ }^{13} \mathrm{C}$ NMR $\left(125 \mathrm{MHz}, \mathrm{CDCl}_{3}\right) \delta_{\mathrm{C}} 165.2,158.6,151.6,144.6,139.1,136.7,136.5,133.5$, 132.1, 131.1, 130.8, 130.0, 129.5, 129.4, 128.2, 126.8, 126.0, 124.3, 122.4, 122.1, 121.3. LRMS (ESI) $[\mathrm{M}+\mathrm{H}]^{+}$found $\mathrm{m} / \mathrm{z}$ 407.3. HRMS (ESI) $[\mathrm{M}+\mathrm{H}]^{+}$found $\mathrm{m} / \mathrm{z}$ 407.0365, calcd for $\mathrm{C}_{22} \mathrm{H}_{13} \mathrm{Cl}_{2} \mathrm{~N}_{2} \mathrm{O}_{2} 407.0354$.<smiles>O=C1c2ccc(Br)cc2Oc2ccc(Cl)cc2N1c1cccc2cccnc12</smiles>

\section{3-bromo-8-chloro-10-(quinolin-8-yl)dibenzo[b,f][1,4] oxazepin-11(10H)-one 7k}

$7 \mathbf{k}$ was obtained as a yellow solid through column chromatography $(\mathrm{PE} / \mathrm{EA}=10 / 1$ to 5/1), yield 40\% (36 mg). 
${ }^{1} \mathrm{H}$ NMR $\left(400 \mathrm{MHz}, \mathrm{CDCl}_{3}\right) \delta_{\mathrm{H}} 8.97(1 \mathrm{H}, \mathrm{dd}, J=4.2,1.7 \mathrm{~Hz}), 8.30(1 \mathrm{H}, \mathrm{dd}, J=8.3,1.7$ Hz), $7.99(1 \mathrm{H}, \mathrm{dd}, J=8.3,1.4 \mathrm{~Hz}), 7.84(1 \mathrm{H}, \mathrm{d}, J=8.3 \mathrm{~Hz}), 7.80(1 \mathrm{H}, \mathrm{dd}, J=7.3,1.4$ Hz), $7.68(1 \mathrm{H}, \mathrm{dd}, J=8.2,7.3 \mathrm{~Hz}), 7.56-7.50(1 \mathrm{H}, \mathrm{m}), 7.50(1 \mathrm{H}, \mathrm{d}, J=2.0 \mathrm{~Hz}), 7.45$ $(1 \mathrm{H}, \mathrm{dd}, J=8.3,1.9 \mathrm{~Hz}), 7.30-7.25(1 \mathrm{H}, \mathrm{m}), 7.06(1 \mathrm{H}, \mathrm{dd}, J=8.6,2.4 \mathrm{~Hz}), 6.64(1 \mathrm{H}$, d, $J=2.4 \mathrm{~Hz}) .{ }^{13} \mathrm{C}$ NMR $\left(125 \mathrm{MHz}, \mathrm{CDCl}_{3}\right) \delta_{\mathrm{C}} 165.7,160.2,151.5,151.3,144.6,139.1$, $136.7,136.5,133.6,130.9,130.1,129.5,129.4,129.0,127.2$, 126.8, 126.0, 125.8, 124.3, 123.3, 122.6, 122.1. LRMS (EI) $[\mathrm{M}]^{+}$found $\mathrm{m} / \mathrm{z}$ 449. HRMS (EI) $[\mathrm{M}]^{+}$found $\mathrm{m} / \mathrm{z}$ 449.9773, calcd for $\mathrm{C}_{22} \mathrm{H}_{12} \mathrm{O}_{2} \mathrm{BrClN}_{2} 449.9770$.<smiles></smiles>

8-chloro-3-methoxy-10-(5-methoxyquinolin-8-yl)dibenzo[b,f][1,4]oxazepin-11(10H)one 7la

7la was obtained as a yellow solid through column chromatography $(\mathrm{PE} / \mathrm{EA}=10 / 1$ to 1/1), yield $57 \%$ (49 mg).

${ }^{1} \mathrm{H}$ NMR $\left(400 \mathrm{MHz}, \mathrm{CDCl}_{3}\right) \delta_{\mathrm{H}} 8.94(1 \mathrm{H}, \mathrm{dd}, J=4.3,1.8 \mathrm{~Hz}), 8.65(1 \mathrm{H}, \mathrm{dd}, J=8.5,1.8$ Hz), $7.88(1 \mathrm{H}, \mathrm{d}, J=8.7 \mathrm{~Hz}), 7.68(1 \mathrm{H}, \mathrm{d}, J=8.2 \mathrm{~Hz}), 7.45(1 \mathrm{H}, \mathrm{dd}, J=8.5,4.2 \mathrm{~Hz})$, $7.23(1 \mathrm{H}, \mathrm{d}, J=8.6 \mathrm{~Hz}), 7.00(1 \mathrm{H}, \mathrm{dd}, J=8.6,2.4 \mathrm{~Hz}), 6.92(1 \mathrm{H}, \mathrm{d}, J=8.3 \mathrm{~Hz}), 6.80$ $(1 \mathrm{H}, \mathrm{dd}, J=8.7,2.5 \mathrm{~Hz}), 6.76(1 \mathrm{H}, \mathrm{d}, J=2.4 \mathrm{~Hz}), 6.65(1 \mathrm{H}, \mathrm{d}, J=2.4 \mathrm{~Hz}), 4.05(3 \mathrm{H}$, s), $3.86(3 \mathrm{H}, \mathrm{s}) .{ }^{13} \mathrm{C} \mathrm{NMR}\left(125 \mathrm{MHz}, \mathrm{CDCl}_{3}\right) \delta_{\mathrm{C}} 165.9,163.6,161.0,155.2,151.1,150.8$, $144.8,136.9,133.3,131.5,130.9,130.0,129.7,124.9,123.6,121.9,121.2,120.5,118.6$, 111.2, 104.2, 103.7, 55.5, 55.2. $[\mathrm{M}+\mathrm{H}]^{+}$found $\mathrm{m} / \mathrm{z}$ 433.2. HRMS (ESI) $[\mathrm{M}+\mathrm{H}]^{+}$found $\mathrm{m} / \mathrm{z} 433.0950$, calcd for $\mathrm{C}_{24} \mathrm{H}_{18} \mathrm{O}_{4} \mathrm{~N}_{2} \mathrm{Cl} 433.0950$.

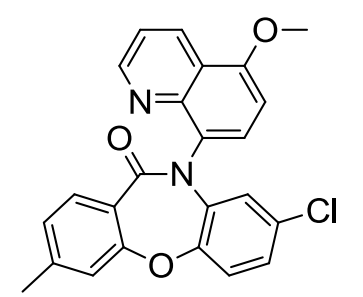

$7 \mathrm{lb}$ 
8-chloro-10-(5-methoxyquinolin-8-yl)-3-methyldibenzo[b,f][1,4]oxazepin-11(10H)-on e $7 \mathbf{l b}$

7lb was obtained as a yellow soild through column chromatography $(\mathrm{PE} / \mathrm{EA}=10 / 1$ to $3 / 1)$, yield $62 \%(51 \mathrm{mg})$.

${ }^{1} \mathrm{H}$ NMR $\left(400 \mathrm{MHz}, \mathrm{CDCl}_{3}\right) \delta_{\mathrm{H}}(1 \mathrm{H}, \mathrm{dd}, J=4.2,1.7 \mathrm{~Hz}), 8.68(1 \mathrm{H}, \mathrm{dd}, J=8.5,1.8 \mathrm{~Hz})$, $7.84(1 \mathrm{H}, \mathrm{d}, J=8.3 \mathrm{~Hz}), 7.70(1 \mathrm{H}, \mathrm{d}, J=8.2 \mathrm{~Hz}), 7.47(1 \mathrm{H}, \mathrm{dd}, J=8.5,4.2 \mathrm{~Hz}), 7.25$ $(1 \mathrm{H}, \mathrm{d}, J=8.6 \mathrm{~Hz}), 7.12-7.07(2 \mathrm{H}, \mathrm{m}), 7.02(1 \mathrm{H}, \mathrm{dd}, J=8.6,2.4 \mathrm{~Hz}), 6.95(1 \mathrm{H}, \mathrm{d}, J$ $=8.2 \mathrm{~Hz}), 6.67(1 \mathrm{H}, \mathrm{d}, J=2.5 \mathrm{~Hz}), 4.08(3 \mathrm{H}, \mathrm{s}), 2.42(3 \mathrm{H}, \mathrm{s}) .{ }^{13} \mathrm{C} \mathrm{NMR}(125 \mathrm{MHz}$, $\left.\mathrm{CDCl}_{3}\right) \quad \delta_{\mathrm{C}} 166.3,159.6,155.2,151.1,144.7,144.3,136.9,131.8,131.6,130.8,129.9$, $129.6,125.9,124.9,123.6,123.4,121.9,121.2,120.5,119.5,103.7,55.5,20.9 .[\mathrm{M}+\mathrm{H}]^{+}$ found $\mathrm{m} / \mathrm{z}$ 417.2 HRMS (ESI) $[\mathrm{M}+\mathrm{H}]^{+}$found $\mathrm{m} / \mathrm{z} 417.0998$, calcd for $\mathrm{C}_{24} \mathrm{H}_{18} \mathrm{O}_{3} \mathrm{~N}_{2} \mathrm{Cl}$ 417.1000.

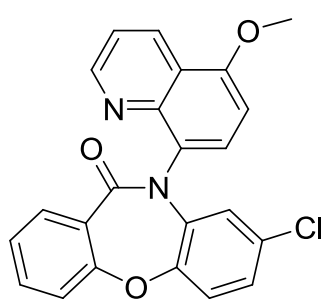

7Ic

\section{8-chloro-10-(5-methoxyquinolin-8-yl)dibenzo[b,f][1,4]oxazepin-11(10H)-one 7lc}

7lc as obtained as a yellow solid through column chromatography (PE/EA = 10/1 to 3/1), yield $75 \%(61 \mathrm{mg})$.

${ }^{1} \mathrm{H}$ NMR $\left(400 \mathrm{MHz}, \mathrm{CDCl}_{3}\right) \delta_{\mathrm{H}} 8.97(1 \mathrm{H}, \mathrm{dd}, J=4.2,1.8 \mathrm{~Hz}), 8.68(1 \mathrm{H}, \mathrm{dd}, J=8.5$, $1.8 \mathrm{~Hz}), 7.96(1 \mathrm{H}, \mathrm{ddd}, J=7.6,1.8,0.8 \mathrm{~Hz}), 7.71(1 \mathrm{H}, \mathrm{d}, J=8.2 \mathrm{~Hz}), 7.55-7.50(1 \mathrm{H}$, m), $7.48(1 \mathrm{H}, \mathrm{dd}, J=8.5,4.2 \mathrm{~Hz}), 7.29(1 \mathrm{H}, \mathrm{d}, J=1.0 \mathrm{~Hz}), 7.28-7.25(2 \mathrm{H}, \mathrm{m}), 7.03$ $(1 \mathrm{H}, \mathrm{dd}, J=8.6,2.5 \mathrm{~Hz}), 6.95(1 \mathrm{H}, \mathrm{d}, J=8.3 \mathrm{~Hz}), 6.67(1 \mathrm{H}, \mathrm{d}, J=2.4 \mathrm{~Hz}), 4.09(3 \mathrm{H}$, s). ${ }^{13} \mathrm{C} \mathrm{NMR}\left(125 \mathrm{MHz}, \mathrm{CDCl}_{3}\right) \delta_{\mathrm{C}} 166.4,159.7,155.3,151.2,151.2,144.7,136.8,133.2$, $132.1,131.5,131.0,130.0,129.6,126.5,125.2,125.1,123.6,122.0,121.3,120.6,119.3$, 103.8, 55.6. LRMS (ESI) $[\mathrm{M}+\mathrm{H}]^{+}$found $\mathrm{m} / \mathrm{z}$ 403.3. HRMS (ESI) $[\mathrm{M}+\mathrm{H}]^{+}$found $\mathrm{m} / \mathrm{z}$ 403.0847, calcd for $\mathrm{C}_{23} \mathrm{H}_{16} \mathrm{O}_{3} \mathrm{~N}_{2} \mathrm{Cl} 403.0844$. 


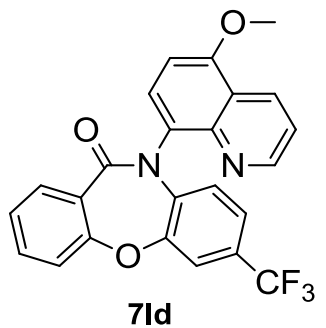

10-(5-methoxyquinolin-8-yl)-7-(trifluoromethyl)dibenzo[b,f][1,4]oxazepin-11(10H)-o ne 7ld

7lc was obtained as a yellow oil through column chromatography (PE/EA = 10/1 to 3/1), yield $79 \%$ (68 mg).

${ }^{1} \mathrm{H}$ NMR $\left(400 \mathrm{MHz}, \mathrm{CDCl}_{3}\right) \quad \delta_{\mathrm{H}} 8.95(1 \mathrm{H}, \mathrm{dd}, J=4.2,1.8 \mathrm{~Hz}), 8.69(1 \mathrm{H}, \mathrm{dd}, J=8.5$, $1.8 \mathrm{~Hz}), 7.98(1 \mathrm{H}, \mathrm{dd}, J=7.7,1.7 \mathrm{~Hz}), 7.71(1 \mathrm{H}, \mathrm{d}, J=8.2 \mathrm{~Hz}), 7.61(1 \mathrm{H}, \mathrm{d}, J=1.5$ Hz), 7.56 (1 H, ddd, $J=8.1,7.3,1.7 \mathrm{~Hz}), 7.48(1 \mathrm{H}, \mathrm{dd}, J=8.5,4.2 \mathrm{~Hz}), 7.37$ - 7.29 (2 H, m), $7.14(1 \mathrm{H}, \mathrm{ddd}, J=8.6,2.2,0.8 \mathrm{~Hz}), 6.95(1 \mathrm{H}, \mathrm{d}, J=8.3 \mathrm{~Hz}), 6.78(1 \mathrm{H}, \mathrm{dd}, J=$ 8.6, 0.9 Hz), 4.08 (3 H, s). ${ }^{13} \mathrm{C}$ NMR (125 MHz, $\left.\mathrm{CDCl}_{3}\right) \quad \delta_{\mathrm{C}} 166.2,159.4,155.3,152.0$, $151.2,144.7,139.0,133.4,132.1,131.5,130.9,129.6,127.0\left(\mathrm{q},{ }^{2} J_{\mathrm{C}-\mathrm{F}}=33.4 \mathrm{~Hz}\right), 126.3$, $125.3,124.0,122.7\left(\mathrm{q},{ }^{1} J_{\mathrm{C}-\mathrm{F}}=272.1 \mathrm{~Hz}\right), 121.8\left(\mathrm{q},{ }^{3} J_{\mathrm{C}-\mathrm{F}}=3.9 \mathrm{~Hz}\right), 121.2,120.6,119.3$, $118.5\left(\mathrm{q},{ }^{2} J_{\mathrm{C}-\mathrm{F}}=3.9 \mathrm{~Hz}\right), 103.7,55.5$. LRMS (ESI) $[\mathrm{M}+\mathrm{H}]^{+}$found $\mathrm{m} / \mathrm{z}$ 437.2. HRMS (ESI) $[\mathrm{M}+\mathrm{H}]^{+}$found $\mathrm{m} / \mathrm{z}$ 437.1107, calcd for $\mathrm{C}_{24} \mathrm{H}_{16} \mathrm{O}_{3} \mathrm{~N}_{2} \mathrm{~F}_{3} 403.1108$.

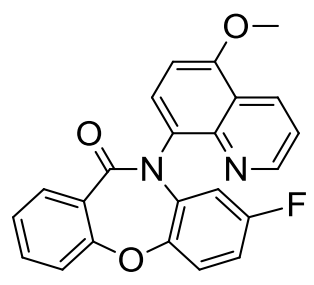

7le

\section{8-fluoro-10-(5-methoxyquinolin-8-yl)dibenzo[b,f] $[1,4]$ oxazepin-11(10H)-one 7le}

7le was obtained as a yellow soild through column chromatography $(\mathrm{PE} / \mathrm{EA}=10 / 1$ to 3/1), yield $80 \%$ (62 mg).

${ }^{1} \mathrm{H}$ NMR $\left(400 \mathrm{MHz}, \mathrm{CDCl}_{3}\right) \quad \delta_{\mathrm{H}} 8.96(1 \mathrm{H}, \mathrm{dd}, J=4.2,1.7 \mathrm{~Hz}), 8.68(1 \mathrm{H}, \mathrm{dd}, J=8.5$, $1.8 \mathrm{~Hz}), 7.97(1 \mathrm{H}, \mathrm{dd}, J=8.1,1.7 \mathrm{~Hz}), 7.71(1 \mathrm{H}, \mathrm{d}, J=8.2 \mathrm{~Hz}), 7.53(1 \mathrm{H}, \mathrm{ddd}, J=8.2$, 7.4, 1.8 Hz), $7.48(1 \mathrm{H}, \mathrm{dd}, J=8.5,4.2 \mathrm{~Hz}), 7.29(2 \mathrm{H}, \mathrm{d}, J=1.0 \mathrm{~Hz}), 7.28-7.24(1 \mathrm{H}$, m), $6.95(1 \mathrm{H}, \mathrm{d}, J=8.2 \mathrm{~Hz}), 6.76(1 \mathrm{H}, \mathrm{ddd}, J=8.9,7.4,3.0 \mathrm{~Hz}), 6.39(1 \mathrm{H}, \mathrm{dd}, J=$ 
10.0, $3.0 \mathrm{~Hz}), 4.09(3 \mathrm{H}, \mathrm{s}) .{ }^{13} \mathrm{C} \mathrm{NMR}\left(125 \mathrm{MHz}, \mathrm{CDCl}_{3}\right) \quad \delta_{\mathrm{C}} 166.8,160.4,159.5(\mathrm{~d}$, $\left.{ }^{1} J_{\mathrm{C}-\mathrm{F}}=243.1 \mathrm{~Hz}\right), 155.7,151.7,149.1\left(\mathrm{~d},{ }^{4} J_{\mathrm{C}-\mathrm{F}}=2.6 \mathrm{~Hz}\right), 145.2,137.3\left(\mathrm{~d},{ }^{3} J_{\mathrm{C}-\mathrm{F}}=10.4\right.$ $\mathrm{Hz}), 133.6,132.6,132.1,131.4,130.0,127.0,125.5,122.1\left(\mathrm{~d},{ }^{3} J_{\mathrm{C}-\mathrm{F}}=9.6 \mathrm{~Hz}\right), 121.7$, 121.0, 119.7, $112.0\left(\mathrm{~d},{ }^{2} J_{\mathrm{C}-\mathrm{F}}=23.4 \mathrm{~Hz}\right), 111.1\left(\mathrm{~d},{ }^{2} J_{\mathrm{C}-\mathrm{F}}=27.0 \mathrm{~Hz}\right), 104.2,56.0 . \mathrm{LRMS}$ (ESI) $[\mathrm{M}+\mathrm{H}]^{+}$found $\mathrm{m} / \mathrm{z}$ 387.2. HRMS (ESI) $[\mathrm{M}+\mathrm{H}]^{+}$found $\mathrm{m} / \mathrm{z}$ 387.1134, calcd for $\mathrm{C}_{23} \mathrm{H}_{16} \mathrm{O}_{3} \mathrm{~N}_{2} \mathrm{~F} 387.1139$.

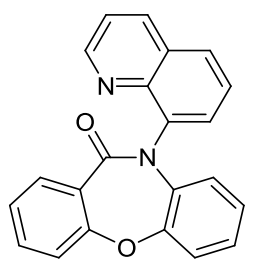

70

\section{0-(quinolin-8-yl)dibenzo[b,f][1,4]oxazepin-11(10H)-one 7o}

7o was obtained as a yellow solid through column chromatography (PE/EA = 10/1 to 5/1), yield 60\% (40 mg).

${ }^{1} \mathrm{H}$ NMR $\left(400 \mathrm{MHz}, \mathrm{CDCl}_{3}\right) \delta_{\mathrm{H}} 8.99(1 \mathrm{H}, \mathrm{dd}, J=4.2,1.7 \mathrm{~Hz}), 8.28(1 \mathrm{H}, \mathrm{dd}, J=8.3,1.7$ Hz), $7.97(2 \mathrm{H}, \mathrm{ddd}, J=16.4,8.0,1.6 \mathrm{~Hz}), 7.82(1 \mathrm{H}, \mathrm{dd}, J=7.4,1.4 \mathrm{~Hz}), 7.73-7.62(1$ H, m), $7.57-7.44(2 \mathrm{H}, \mathrm{m}), 7.42-7.23(3 \mathrm{H}, \mathrm{m}), 7.13-6.99(1 \mathrm{H}, \mathrm{m}), 6.89$ (1 H, ddd, $J$ $=8.6,7.5,1.5 \mathrm{~Hz}), 6.66(1 \mathrm{H}, \mathrm{dd}, J=8.2,1.5 \mathrm{~Hz}) .{ }^{13} \mathrm{C} \mathrm{NMR}\left(125 \mathrm{MHz}, \mathrm{CDCl}_{3}\right) \delta_{\mathrm{C}} 166.3$, $160.1,152.8,150.9,144.6,139.6,135.9,135.5,133.1,132.0,129.7,128.9,128.4,126.7$, $126.4,125.4,125.0,124.9,123.8,121.4,121.0,119.4 . \mathrm{LRMS}(\mathrm{ESI})[\mathrm{M}+\mathrm{H}]^{+}$found $\mathrm{m} / \mathrm{z}$ 339.3. HRMS (ESI) $[\mathrm{M}+\mathrm{H}]^{+}$found $\mathrm{m} / \mathrm{z} 339.1128$, calcd for $\mathrm{C}_{22} \mathrm{H}_{15} \mathrm{O}_{2} \mathrm{~N}_{2} 339.1128$.

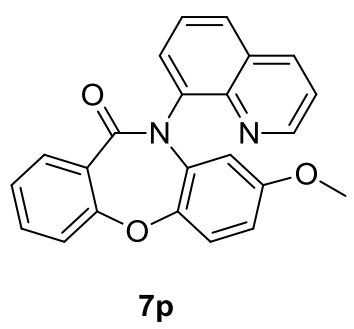

\section{8-methoxy-10-(quinolin-8-yl)dibenzo[b,f][1,4]oxazepin-11(10H)-one 7p}

$7 \mathbf{p}$ was obtained as a yellow solid through column chromatography $(\mathrm{PE} / \mathrm{EA}=10 / 1$ to 3/1), yield 48\% (35 mg). 
${ }^{1} \mathrm{H}$ NMR $\left(400 \mathrm{MHz}, \mathrm{CDCl}_{3}\right) \delta_{\mathrm{H}} 8.99(1 \mathrm{H}, \mathrm{dd}, J=4.4,1.7 \mathrm{~Hz}), 8.36-8.13(1 \mathrm{H}, \mathrm{m}), 8.08$ $-7.89(2 \mathrm{H}, \mathrm{m}), 7.82(1 \mathrm{H}, \mathrm{dd}, J=7.4,1.4 \mathrm{~Hz}), 7.67(1 \mathrm{H}, \mathrm{d}, J=7.7 \mathrm{~Hz}), 7.59-7.41(2$ H, m), $7.30(1 \mathrm{H}, \mathrm{s}), 7.27(2 \mathrm{H}, \mathrm{d}, J=5.8 \mathrm{~Hz}), 6.60(1 \mathrm{H}, \mathrm{dd}, J=8.9,2.9 \mathrm{~Hz}), 6.20(1 \mathrm{H}$, d, $J=2.9 \mathrm{~Hz}), 3.49(3 \mathrm{H}, \mathrm{s}) .{ }^{13} \mathrm{C}$ NMR $\left(125 \mathrm{MHz}, \mathrm{CDCl}_{3}\right) \delta_{\mathrm{C}} 166.9,160.7,156.7,151.4$, $147.3,144.9,139.9,136.5,136.4,133.6,132.4,130.1,129.4,128.9,127.2,126.9,125.3$, 121.9, 121.7, 119.7, 110.6, 110.0, 55.5. LRMS $[\mathrm{M}+\mathrm{H}]^{+}$found $\mathrm{m} / \mathrm{z} 369.2$ HRMS (ESI) $[\mathrm{M}+\mathrm{H}]^{+}$found $\mathrm{m} / \mathrm{z} 369.1245$, calcd for $\mathrm{C}_{23} \mathrm{H}_{17} \mathrm{O}_{3} \mathrm{~N}_{2} 369.1234$.

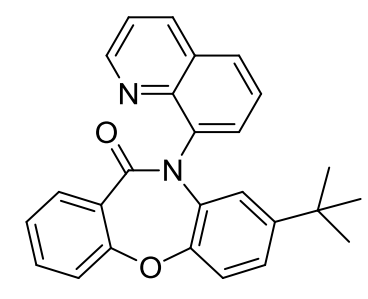

$7 q$

\section{8-(tert-butyl)-10-(quinolin-8-yl)dibenzo[b,f][1,4]oxazepin-11(10H)-one 7q}

7q was obtained as a yellow solid through column chromatography $(\mathrm{PE} / \mathrm{EA}=10 / 1$ to $5 / 1)$, yield 50\% (39 mg).

${ }^{1} \mathrm{H}$ NMR $\left(400 \mathrm{MHz}, \mathrm{CDCl}_{3}\right) \delta_{\mathrm{H}} 9.01(1 \mathrm{H}, \mathrm{dd}, J=4.2,1.7 \mathrm{~Hz}), 8.28(1 \mathrm{H}, \mathrm{dd}, J=8.3,1.7$ $\mathrm{Hz}), 8.00(1 \mathrm{H}, \mathrm{dd}, J=7.7,1.7 \mathrm{~Hz}), 7.95(1 \mathrm{H}, \mathrm{dd}, J=8.2,1.4 \mathrm{~Hz}), 7.83(1 \mathrm{H}, \mathrm{dd}, J=7.3$, $1.4 \mathrm{~Hz}), 7.73-7.63(1 \mathrm{H}, \mathrm{m}), 7.56$ - $7.44(2 \mathrm{H}, \mathrm{m}), 7.33-7.29(2 \mathrm{H}, \mathrm{m}), 7.28-7.24$ (1 $\mathrm{H}, \mathrm{m}), 7.10(1 \mathrm{H}, \mathrm{dd}, J=8.5,2.3 \mathrm{~Hz}), 6.69(1 \mathrm{H}, \mathrm{d}, J=2.3 \mathrm{~Hz}), 0.97(9 \mathrm{H}, \mathrm{s}) .{ }^{13} \mathrm{C} \mathrm{NMR}$ $\left(125 \mathrm{MHz}, \mathrm{CDCl}_{3}\right) \delta_{\mathrm{C}} 166.9,160.6,151.3,150.9,148.4,145.1,140.2,136.4,135.0$, $133.5,132.5,130.1,129.2,128.8,127.2,126.9,125.2,122.9,121.7,121.3,120.7,119.8$, 34.2, 31.0. LRMS (ESI) $[\mathrm{M}+\mathrm{H}]^{+}$found $\mathrm{m} / \mathrm{z}$ 395.1. HRMS (ESI) $[\mathrm{M}+\mathrm{H}]^{+}$found $\mathrm{m} / \mathrm{z}$ 395.1755, calcd for $\mathrm{C}_{26} \mathrm{H}_{23} \mathrm{O}_{2} \mathrm{~N}_{2} 395.1754$.

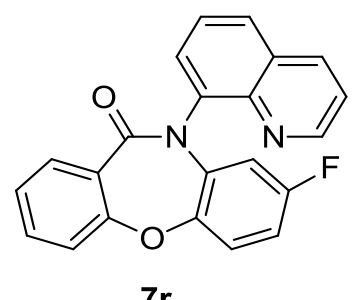

$7 r$

8-fluoro-10-(quinolin-8-yl)dibenzo[b,f][1,4] oxazepin-11(10H)-one 7r 
$7 \mathbf{r}$ was obtained as a yellow solid through column chromatography $(\mathrm{PE} / \mathrm{EA}=10 / 1$ to $5 / 1)$, yield $82 \%$ (58 mg).

${ }^{1} \mathrm{H}$ NMR $\left(400 \mathrm{MHz}, \mathrm{CDCl}_{3}\right) \delta_{\mathrm{H}} 8.99(1 \mathrm{H}, \mathrm{dd}, J=4.2,1.7 \mathrm{~Hz}), 8.28(1 \mathrm{H}, \mathrm{dd}, J=8.3,1.7$ Hz), $7.98(2 \mathrm{H}$, ddd, $J=12.3,8.1,1.6 \mathrm{~Hz}), 7.82(1 \mathrm{H}, \mathrm{dd}, J=7.4,1.4 \mathrm{~Hz}), 7.67(1 \mathrm{H}, \mathrm{t}, J$ $=7.8 \mathrm{~Hz}), 7.58-7.42(2 \mathrm{H}, \mathrm{m}), 7.34-7.22(3 \mathrm{H}, \mathrm{m}), 6.77(1 \mathrm{H}, \mathrm{ddd}, J=8.9,7.4,3.0 \mathrm{~Hz})$, $6.37(1 \mathrm{H}, \mathrm{dd}, J=9.9,3.0 \mathrm{~Hz}) .{ }^{13} \mathrm{C}$ NMR $\left(125 \mathrm{MHz}, \mathrm{CDCl}_{3}\right) \delta_{\mathrm{C}} 166.6,160.4,159.5(\mathrm{~d}$, $\left.{ }^{1} J_{\mathrm{C}-\mathrm{F}}=253.0 \mathrm{~Hz}\right), 151.5,149.2\left(\mathrm{~d},{ }^{4} J_{\mathrm{C}-\mathrm{F}}=2.9 \mathrm{~Hz}\right), 144.8,139.5,137.0\left(\mathrm{~d},{ }^{3} J_{\mathrm{C}-\mathrm{F}}=10.4\right.$ $\mathrm{Hz}), 136.5,133.8,132.5,130.1,129.5,129.2,126.9,126.9,125.6,122.3\left(\mathrm{~d},{ }^{3} J_{\mathrm{C}-\mathrm{F}}=9.6\right.$ $\mathrm{Hz}), 122.0,119.8,112.24\left(\mathrm{~d},{ }^{2} J_{\mathrm{C}-\mathrm{F}}=23.3 \mathrm{~Hz}\right), 111.20\left(\mathrm{~d},{ }^{2} J_{\mathrm{C}-\mathrm{F}}=27.0 \mathrm{~Hz}\right)$. LRMS (ESI) $[\mathrm{M}+\mathrm{H}]^{+}$found $\mathrm{m} / \mathrm{z} 357.2$ HRMS (ESI) $[\mathrm{M}+\mathrm{H}]^{+}$found $\mathrm{m} / \mathrm{z}$ 357.1036, calcd for $\mathrm{C}_{22} \mathrm{H}_{14} \mathrm{O}_{2} \mathrm{~N}_{2} \mathrm{~F} 357.1034$.

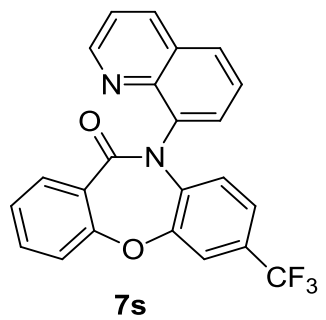

\section{0-(quinolin-8-yl)-7-(trifluoromethyl)dibenzo[b,f][1,4]oxazepin-11(10H)-one 7s}

$7 \mathbf{s}$ was obtained as a yellow solid through column chromatography (PE/EA $=10 / 1$ to $5 / 1$ ), yield $80 \%$ ( $65 \mathrm{mg}$ ).

${ }^{1} \mathrm{H}$ NMR $\left(400 \mathrm{MHz}, \mathrm{CDCl}_{3}\right) \delta_{\mathrm{H}} 8.97(1 \mathrm{H}, \mathrm{dd}, J=4.2,1.7 \mathrm{~Hz}), 8.30(1 \mathrm{H}, \mathrm{dd}, J=8.4,1.7$ Hz), $7.99(2 \mathrm{H}, \mathrm{dd}, J=8.0,1.5 \mathrm{~Hz}), 7.82(1 \mathrm{H}, \mathrm{dd}, J=7.3,1.4 \mathrm{~Hz}), 7.69(1 \mathrm{H}, \mathrm{dd}, J=8.2$, $7.3 \mathrm{~Hz}), 7.63(1 \mathrm{H}, \mathrm{d}, J=2.1 \mathrm{~Hz}), 7.57(1 \mathrm{H}, \mathrm{ddd}, J=8.1,7.3,1.8 \mathrm{~Hz}), 7.51(1 \mathrm{H}, \mathrm{dd}, J=$ 8.3, 4.2 Hz), $7.40-7.30(2 \mathrm{H}, \mathrm{m}), 7.18-7.11(1 \mathrm{H}, \mathrm{m}), 6.73(1 \mathrm{H}, \mathrm{dd}, J=8.4,1.1 \mathrm{~Hz})$. ${ }^{13} \mathrm{C}$ NMR $\left(125 \mathrm{MHz}, \mathrm{CDCl}_{3}\right) \delta_{\mathrm{C}} 166.5,159.9,152.6,151.5,144.7,139.4,139.1,136.4$, 134.0, 132.6, 130.1, 129.5, 129.3, $127.7\left(\mathrm{q},{ }^{2} J_{\mathrm{C}-\mathrm{F}}=33.4 \mathrm{~Hz}\right), 126.8,126.7,125.8,124.5$, $123.4\left(\mathrm{q},{ }^{1} J_{\mathrm{C}-\mathrm{F}}=272.1 \mathrm{~Hz}\right), 122.4\left(\mathrm{q},{ }^{3} J_{\mathrm{C}-\mathrm{F}}=3.9 \mathrm{~Hz}\right), 122.0,119.9,119.1\left(\mathrm{q},{ }^{3} J_{\mathrm{C}-\mathrm{F}}=3.9\right.$ Hz). LRMS (ESI) $[\mathrm{M}+\mathrm{H}]^{+}$found $\mathrm{m} / \mathrm{z}$ 407.3 HRMS (ESI) $[\mathrm{M}+\mathrm{H}]^{+}$found $\mathrm{m} / \mathrm{z}$ 407.0990, calcd for $\mathrm{C}_{23} \mathrm{H}_{14} \mathrm{O}_{2} \mathrm{~N}_{2} \mathrm{~F}_{3} 407.1002$. 
<smiles>O=C1c2ccccc2Oc2cc(F)c(F)cc2N1c1cccc2cccnc12</smiles>

\section{7,8-difluoro-10-(quinolin-8-yl)dibenzo[b,f][1,4]oxazepin-11(10H)-one $7 t$}

$7 \mathbf{t}$ was obtained as a brown solid through column chromatography ( $\mathrm{PE} / \mathrm{EA}=10 / 1$ to $5 / 1$ ), yield $84 \%(63 \mathrm{mg})$.

${ }^{1} \mathrm{H}$ NMR $\left(400 \mathrm{MHz}, \mathrm{CDCl}_{3}\right) \delta_{\mathrm{H}} 8.98(1 \mathrm{H}, \mathrm{dd}, J=4.2,1.7 \mathrm{~Hz}), 8.30(1 \mathrm{H}, \mathrm{dd}, J=8.4,1.7$ Hz), $7.98(2 \mathrm{H}, \mathrm{dt}, J=7.7,1.6 \mathrm{~Hz}), 7.82(1 \mathrm{H}, \mathrm{dd}, J=7.3,1.4 \mathrm{~Hz}), 7.69(1 \mathrm{H}, \mathrm{dd}, J=8.2$, $7.3 \mathrm{~Hz}), 7.61-7.47(2 \mathrm{H}, \mathrm{m}), 7.36-7.29(2 \mathrm{H}, \mathrm{m}), 7.28(0 \mathrm{H}, \mathrm{d}, J=1.2 \mathrm{~Hz}), 7.20(1 \mathrm{H}$, dd, $J=10.0,7.5 \mathrm{~Hz}), 6.51(1 \mathrm{H}, \mathrm{dd}, J=11.5,8.0 \mathrm{~Hz}) .{ }^{13} \mathrm{C} \mathrm{NMR}\left(125 \mathrm{MHz}, \mathrm{CDCl}_{3}\right) \delta_{\mathrm{C}}$ 166.3, 160.1, 151.6, $148.8\left(\mathrm{dd}, J_{\mathrm{C}-\mathrm{F}}=2.6 \mathrm{~Hz}, 8.2 \mathrm{~Hz}\right), 147.3\left(\mathrm{dd}, J_{\mathrm{C}-\mathrm{F}}=245.3 \mathrm{~Hz}, 13.3\right.$ $\mathrm{Hz}), 147.1\left(\mathrm{dd}, J_{\mathrm{C}-\mathrm{F}}=249.7 \mathrm{~Hz}, 13.9 \mathrm{~Hz}\right), 144.6,139.4,136.5,133.8,132.6,132.4(\mathrm{dd}$, $\left.J_{\mathrm{C}-\mathrm{F}}=3.4 \mathrm{~Hz}, 7.6 \mathrm{~Hz}\right), 130.1,129.5,129.3,126.9,126.6,125.8,122.0,119.7,112.7(\mathrm{~d}$, $\left.J_{\mathrm{C}-\mathrm{F}}=21.83 \mathrm{~Hz}\right), 110.4\left(\mathrm{~d}, J_{\mathrm{C}-\mathrm{F}}=19.4 \mathrm{~Hz}\right) \mathrm{LRMS}(\mathrm{ESI})[\mathrm{M}+\mathrm{H}]^{+}$found $\mathrm{m} / \mathrm{z} 375.7 \mathrm{HRMS}$ (ESI) $[\mathrm{M}+\mathrm{H}]^{+}$found $\mathrm{m} / \mathrm{z}$ 375.0929, calcd for $\mathrm{C}_{22} \mathrm{H}_{13} \mathrm{O}_{2} \mathrm{~N}_{2} \mathrm{~F}_{2} 375.0940$.

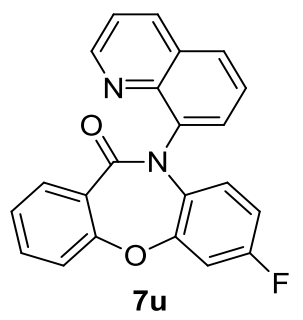

\section{7-fluoro-10-(quinolin-8-yl)dibenzo[b,f][1,4]oxazepin-11(10H)-one 7u}

$7 \mathbf{u}$ was obtained as a yellow solid through column chromatography (PE/EA $=10 / 1$ to 5/1), yield 73\% (52 mg).

${ }^{1} \mathrm{H}$ NMR $\left(400 \mathrm{MHz}, \mathrm{CDCl}_{3}\right) \delta_{\mathrm{H}} 8.99(1 \mathrm{H}, \mathrm{dd}, J=4.2,1.7 \mathrm{~Hz}), 8.28(1 \mathrm{H}, \mathrm{dd}, J=8.3,1.7$ Hz), $7.97(2 \mathrm{H}$, ddd, $J=13.8,7.9,1.5 \mathrm{~Hz}), 7.81(1 \mathrm{H}, \mathrm{dd}, J=7.3,1.4 \mathrm{~Hz}), 7.67(1 \mathrm{H}, \mathrm{dd}$, $J=8.2,7.3 \mathrm{~Hz}), 7.60-7.44(2 \mathrm{H}, \mathrm{m}), 7.41-7.24(2 \mathrm{H}, \mathrm{m}), 7.10(1 \mathrm{H}, \mathrm{dd}, J=8.5,2.6$ $\mathrm{Hz}), 6.74-6.53(2 \mathrm{H}, \mathrm{m}) .{ }^{13} \mathrm{C} \mathrm{NMR}\left(125 \mathrm{MHz}, \mathrm{CDCl}_{3}\right) \delta_{\mathrm{C}} 165.9,159.7,159.3\left(\mathrm{~d},{ }^{1} J_{\mathrm{C}-\mathrm{F}}=\right.$ $247.2 \mathrm{~Hz}), 153.5\left(\mathrm{~d},{ }^{3} J_{\mathrm{C}-\mathrm{F}}=10.6 \mathrm{~Hz}\right), 151.0,144.4,139.5,136.0,133.2,132.1,131.9(\mathrm{~d}$, 
$\left.{ }^{4} J_{\mathrm{C}-\mathrm{F}}=3.7 \mathrm{~Hz}\right), 129.7,129.0,128.5,126.4,125.3,124.7\left(\mathrm{~d},{ }^{3} J_{\mathrm{C}-\mathrm{F}}=9.6 \mathrm{~Hz}\right), 121.5,119.4$, $112.0\left(\mathrm{~d},{ }^{2} J_{\mathrm{C}-\mathrm{F}}=22.4 \mathrm{~Hz}\right), 118.5\left(\mathrm{~d},{ }^{2} J_{\mathrm{C}-\mathrm{F}}=24.0 \mathrm{~Hz}\right)$. LRMS $(\mathrm{ESI})[\mathrm{M}+\mathrm{H}]^{+}$found $\mathrm{m} / \mathrm{z}$ 357.2 HRMS (ESI) $[\mathrm{M}+\mathrm{H}]^{+}$found m/z 357.1034, calcd for $\mathrm{C}_{22} \mathrm{H}_{14} \mathrm{O}_{2} \mathrm{~N}_{2} \mathrm{~F} 357.1034$.

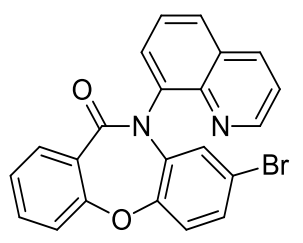

$7 w$

\section{8-bromo-10-(quinolin-8-yl)dibenzo[b,f][1,4]oxazepin-11(10H)-one 7w}

$7 \mathbf{w}$ was obtained as a yellow solid through column chromatography (PE/EA $=10 / 1$ to $5 / 1)$, yield $69 \%(58 \mathrm{mg})$.

${ }^{1} \mathrm{H}$ NMR $\left(400 \mathrm{MHz}, \mathrm{CDCl}_{3}\right) \delta_{\mathrm{H}} 8.99(1 \mathrm{H}, \mathrm{dd}, J=4.2,1.7 \mathrm{~Hz}), 8.29(1 \mathrm{H}, \mathrm{dd}, J=8.4,1.7$ Hz), $7.97(2 \mathrm{H}, \mathrm{ddd}, J=7.9,3.6,1.4 \mathrm{~Hz}), 7.82(1 \mathrm{H}, \mathrm{dd}, J=7.3,1.4 \mathrm{~Hz}), 7.69(1 \mathrm{H}, \mathrm{dd}, J$ $=8.2,7.3 \mathrm{~Hz}), 7.60-7.44(2 \mathrm{H}, \mathrm{m}), 7.34-7.28(2 \mathrm{H}, \mathrm{m}), 7.24(1 \mathrm{H}, \mathrm{d}, J=8.6 \mathrm{~Hz}), 7.19$ $(1 \mathrm{H}, \mathrm{dd}, J=8.6,2.2 \mathrm{~Hz}), 6.78(1 \mathrm{H}, \mathrm{d}, J=2.2 \mathrm{~Hz}) .{ }^{13} \mathrm{C}$ NMR $\left(125 \mathrm{MHz}, \mathrm{CDCl}_{3}\right) \delta_{\mathrm{C}}$ $166.5,160.2,152.4,151.5,144.7,139.4,137.3,136.5,133.8,132.5,130.2,129.5,129.3$, $128.8,127.0,126.8,126.8,125.7,122.9,122.0,119.8,118.0$. LRMS $(\mathrm{ESI})[\mathrm{M}+\mathrm{H}]^{+}$found $\mathrm{m} / \mathrm{z}$ 417.0 HRMS (ESI) $[\mathrm{M}+\mathrm{H}]^{+}$found $\mathrm{m} / \mathrm{z}$ 417.0238, calcd for $\mathrm{C}_{22} \mathrm{H}_{14} \mathrm{O}_{2} \mathrm{~N}_{2} \mathrm{Br} 417.0233$.<smiles>O=C1c2ccccc2Oc2cc(Cl)ccc2N1c1cccc2cccnc12</smiles>

$8 a$

\section{7-chloro-10-(quinolin-8-yl)dibenzo[b,f][1,4]oxazepin-11(10H)-one 8a}

8a was obtained as a yellow solid through column chromatography (PE/EA = 10/1 to 5/1), yield $70 \%$ (52 mg).

${ }^{1} \mathrm{H}$ NMR $\left(400 \mathrm{MHz}, \mathrm{CDCl}_{3}\right) \delta_{\mathrm{H}} 9.19-8.79(1 \mathrm{H}, \mathrm{m}), 8.32-8.23(1 \mathrm{H}, \mathrm{m}), 8.05-7.87(2$ H, m), $7.81(1 \mathrm{H}, \mathrm{dt}, J=7.3,1.1 \mathrm{~Hz}), 7.67(1 \mathrm{H}, \mathrm{t}, J=7.8 \mathrm{~Hz}), 7.61-7.44(2 \mathrm{H}, \mathrm{m}), 7.40$ - 7.29 (3 H, m), $6.94-6.80(1 \mathrm{H}, \mathrm{m}), 6.59(1 \mathrm{H}, \mathrm{dd}, J=8.8,0.9 \mathrm{~Hz}) .{ }^{13} \mathrm{C}$ NMR $(125$ 
$\left.\mathrm{MHz}, \mathrm{CDCl}_{3}\right) \delta_{\mathrm{C}} \quad 166.4,160.1,153.4,151.4,144.8,139.7,136.4,134.8,133.7,132.5$, $130.5,130.1,129.4,129.1,126.8,125.7,125.6,125.1,121.9,121.8,119.9$. LRMS (ESI) $[\mathrm{M}+\mathrm{H}]^{+}$found $\mathrm{m} / \mathrm{z} 373.3$ HRMS (ESI) $[\mathrm{M}+\mathrm{H}]^{+}$found $\mathrm{m} / \mathrm{z}$ 373.0741, calcd for $\mathrm{C}_{22} \mathrm{H}_{14} \mathrm{O}_{2} \mathrm{~N}_{2} \mathrm{Cl} 373.0738$.<smiles>COc1ccc2c(c1)Oc1cc(Cl)ccc1N(c1cccc3cccnc13)C2=O</smiles>

$8 b$

7-chloro-3-methoxy-10-(quinolin-8-yl)dibenzo[b,f][1,4] oxazepin-11(10H)-one 8b

8b was obtained as a yellow solid through column chromatography $(\mathrm{PE} / \mathrm{EA}=10 / 1$ to 5/1), yield 58\% (47 mg).

${ }^{1} \mathrm{H}$ NMR $\left(400 \mathrm{MHz}, \mathrm{CDCl}_{3}\right) \delta_{\mathrm{H}} 8.97(1 \mathrm{H}, \mathrm{dd}, J=4.2,1.7 \mathrm{~Hz}), 8.27(1 \mathrm{H}, \mathrm{dd}, J=8.3,1.7$ Hz), $7.95(1 \mathrm{H}, \mathrm{dd}, J=8.3,1.4 \mathrm{~Hz}), 7.91(1 \mathrm{H}, \mathrm{d}, J=8.7 \mathrm{~Hz}), 7.80(1 \mathrm{H}, \mathrm{dd}, J=7.3,1.4$ $\mathrm{Hz}), 7.66(1 \mathrm{H}, \mathrm{dd}, J=8.2,7.3 \mathrm{~Hz}), 7.49(1 \mathrm{H}, \mathrm{dd}, J=8.3,4.2 \mathrm{~Hz}), 7.35(1 \mathrm{H}, \mathrm{d}, J=2.4$ Hz), $6.85(2 \mathrm{H}, \mathrm{ddd}, J=8.6,5.1,2.4 \mathrm{~Hz}), 6.80(1 \mathrm{H}, \mathrm{d}, J=2.4 \mathrm{~Hz}), 6.59(1 \mathrm{H}, \mathrm{d}, J=8.8$ $\mathrm{Hz}), 3.90(3 \mathrm{H}, \mathrm{s}) .{ }^{13} \mathrm{C}$ NMR $\left(125 \mathrm{MHz}, \mathrm{CDCl}_{3}\right) \delta_{\mathrm{C}} 167.4,165.5,162.7,154.4,152.8$, $146.3,141.1,137.8,136.3,135.2,131.7,131.6,130.8,130.4,128.1,127.0,126.4,123.2$, 123.1, 120.3, 113.3, 106.1, 57.1. LRMS (ESI) $[\mathrm{M}+\mathrm{H}]^{+}$found $\mathrm{m} / \mathrm{z} 403.3$ HRMS (ESI) $[\mathrm{M}+\mathrm{Na}]^{+}$found $\mathrm{m} / \mathrm{z} 425.0663$ calcd for $\mathrm{C}_{23} \mathrm{H}_{15} \mathrm{O}_{3} \mathrm{~N}_{2} \mathrm{ClNa} 425.0669$.

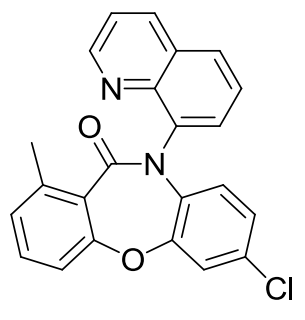

8c

\section{7-chloro-1-methyl-10-(quinolin-8-yl)dibenzo[b,f][1,4]oxazepin-11(10H)-one 8c}

8c was obtained as a yellow solid through column chromatography $(\mathrm{PE} / \mathrm{EA}=10 / 1$ to $5 / 1)$, yield 69\% (53 mg). 
${ }^{1} \mathrm{H}$ NMR $\left(400 \mathrm{MHz}, \mathrm{CDCl}_{3}\right) \delta_{\mathrm{H}} 8.97(1 \mathrm{H}, \mathrm{s}), 8.28(1 \mathrm{H}, \mathrm{d}, J=8.0 \mathrm{~Hz}), 7.93(1 \mathrm{H}, \mathrm{dd}, J=$ 8.1, 1.6 Hz), $7.78-7.59(2 \mathrm{H}, \mathrm{m}), 7.51(1 \mathrm{H}, \mathrm{s}), 7.39-7.28(2 \mathrm{H}, \mathrm{m}), 7.20-7.06(2 \mathrm{H}$, m), $6.83(1 \mathrm{H}, \mathrm{dd}, J=8.8,2.4 \mathrm{~Hz}), 6.42(1 \mathrm{H}, \mathrm{d}, J=8.9 \mathrm{~Hz}), 2.57(3 \mathrm{H}, \mathrm{s}) .{ }^{13} \mathrm{C} \mathrm{NMR}(125$ $\left.\mathrm{MHz}, \mathrm{CDCl}_{3}\right) \delta_{\mathrm{C}} \quad 168.4,162.3,155.2,152.7,146.0,143.6,141.6,137.9,136.5,133.0$, $131.5,131.2,130.7,130.2,129.9,128.5,127.9,127.0,126.0,123.5,122.9,118.4,22.0$. LRMS (ESI) $[\mathrm{M}+\mathrm{H}]^{+}$found m/z 409.1 HRMS (ESI) $[\mathrm{M}+\mathrm{Na}]^{+}$found m/z 409.0717 calcd for $\mathrm{C}_{23} \mathrm{H}_{15} \mathrm{O}_{2} \mathrm{~N}_{2} \mathrm{ClNa} 409.0720$.

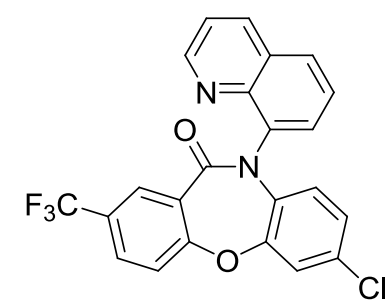

8d

7-chloro-10-(quinolin-8-yl)-2-(trifluoromethyl)dibenzo[b,f][1,4]oxazepin-11(10H)-on e 8d

8d was obtained as a yellow solid through column chromatography $(\mathrm{PE} / \mathrm{EA}=10 / 1$ to 5/1), yield 70\% (62 mg).

${ }^{1} \mathrm{H}$ NMR $\left(400 \mathrm{MHz}, \mathrm{CDCl}_{3}\right) \delta_{\mathrm{H}} 8.98(1 \mathrm{H}, \mathrm{dd}, J=4.2,1.7 \mathrm{~Hz}), 8.35-8.24(2 \mathrm{H}, \mathrm{m}), 7.99$ $(1 \mathrm{H}, \mathrm{dd}, J=8.3,1.4 \mathrm{~Hz}), 7.80(2 \mathrm{H}, \mathrm{dt}, J=8.4,2.1 \mathrm{~Hz}), 7.69(1 \mathrm{H}, \mathrm{dd}, J=8.2,7.3 \mathrm{~Hz})$, $7.52(1 \mathrm{H}, \mathrm{dd}, J=8.3,4.2 \mathrm{~Hz}), 7.46-7.40(1 \mathrm{H}, \mathrm{m}), 7.38(1 \mathrm{H}, \mathrm{d}, J=2.4 \mathrm{~Hz}), 6.90(1 \mathrm{H}$, dd, $J=8.8,2.4 \mathrm{~Hz}), 6.60(1 \mathrm{H}, \mathrm{d}, J=8.8 \mathrm{~Hz}) .{ }^{13} \mathrm{C} \mathrm{NMR}\left(125 \mathrm{MHz}, \mathrm{CDCl}_{3}\right) \delta_{\mathrm{C}} 165.1$, $162.2,152.7,151.6,144.6,139.2,136.5,134.3,130.9,130.6\left(\mathrm{q},{ }^{3} J_{\mathrm{C}-\mathrm{F}}=3.5 \mathrm{~Hz}\right), 130.4(\mathrm{q}$, $\left.{ }^{3} J_{\mathrm{C}-\mathrm{F}}=4.1 \mathrm{~Hz}\right), 130.0,129.5,129.3,128.3\left(\mathrm{q},{ }^{2} J_{\mathrm{C}-\mathrm{F}}=33.5 \mathrm{~Hz}\right), 127.5,126.8,126.2,125.3$, $123.5\left(\mathrm{q},{ }^{1} J_{\mathrm{C}-\mathrm{F}}=272.2 \mathrm{~Hz}\right), 122.1,121.8,120.8$. LRMS $(\mathrm{ESI})[\mathrm{M}+\mathrm{H}]^{+}$found $\mathrm{m} / \mathrm{z} 441.2$ HRMS (ESI) $[\mathrm{M}+\mathrm{H}]^{+}$found $\mathrm{m} / \mathrm{z} 441.0608$ calcd for $\mathrm{C}_{23} \mathrm{H}_{13} \mathrm{O}_{2} \mathrm{~N}_{2} \mathrm{ClF}_{3} 441.0618$. 


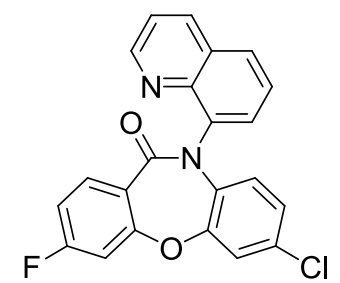

$8 e$

\section{7-chloro-3-fluoro-10-(quinolin-8-yl)dibenzo[b,f][1,4]oxazepin-11(10H)-one 8e}

8e was obtained as a yellow solid through column chromatography (PE/EA = 10/1 to 5/1), yield $75 \%(59 \mathrm{mg})$.

${ }^{1} \mathrm{H}$ NMR $\left(400 \mathrm{MHz}, \mathrm{CDCl}_{3}\right) \delta_{\mathrm{H}} 8.97(1 \mathrm{H}, \mathrm{dd}, J=4.2,1.7 \mathrm{~Hz}), 8.28(1 \mathrm{H}, \mathrm{dd}, J=8.3,1.7$ Hz), $8.06-7.92(2 \mathrm{H}, \mathrm{m}), 7.80(1 \mathrm{H}, \mathrm{dd}, J=7.3,1.4 \mathrm{~Hz}), 7.67(1 \mathrm{H}, \mathrm{dd}, J=8.2,7.3 \mathrm{~Hz})$, $7.50(1 \mathrm{H}, \mathrm{dd}, J=8.3,4.2 \mathrm{~Hz}), 7.36(1 \mathrm{H}, \mathrm{d}, J=2.4 \mathrm{~Hz}), 7.13-6.95(2 \mathrm{H}, \mathrm{m}), 6.88(1 \mathrm{H}$, ddd, $J=8.9,2.4,0.6 \mathrm{~Hz}), 6.60(1 \mathrm{H}, \mathrm{d}, J=8.8 \mathrm{~Hz}) .{ }^{13} \mathrm{C} \mathrm{NMR}\left(100 \mathrm{MHz}, \mathrm{CDCl}_{3}\right) \delta_{\mathrm{C}}$ $165.1\left(\mathrm{~d},{ }^{1} J_{\mathrm{C}-\mathrm{F}}=255.3 \mathrm{~Hz}\right), 165.0,160.6\left(\mathrm{~d},{ }^{3} J_{\mathrm{C}-\mathrm{F}}=11.5 \mathrm{~Hz}\right), 152.4,151.0,144.3,139.0$, 136.0, 134.1, $133.9\left(\mathrm{~d},{ }^{3} J_{\mathrm{C}-\mathrm{F}}=10.4 \mathrm{~Hz}\right), 130.2,129.7,129.0,128.7,126.4,125.5,124.7$, $122.6\left(\mathrm{~d},{ }^{4} J_{\mathrm{C}-\mathrm{F}}=3.5 \mathrm{~Hz}\right), 121.5,121.3,112.8\left(\mathrm{~d},{ }^{2} J_{\mathrm{C}-\mathrm{F}}=21.6 \mathrm{~Hz}\right), 107.1\left(\mathrm{~d},{ }^{2} J_{\mathrm{C}-\mathrm{F}}=23.7\right.$ Hz). LRMS (ESI) $[\mathrm{M}+\mathrm{H}]^{+}$found m/z $391.3 \mathrm{HRMS}(\mathrm{ESI})[\mathrm{M}+\mathrm{H}]^{+}$found $\mathrm{m} / \mathrm{z} 391.0649$ calcd for $\mathrm{C}_{22} \mathrm{H}_{13} \mathrm{O}_{2} \mathrm{~N}_{2} \mathrm{ClF} 391.0650$.

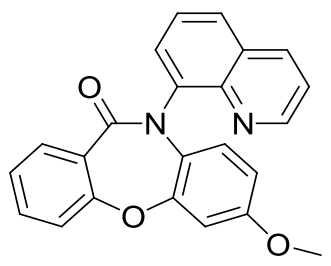

$8 h$

\section{7-methoxy-10-(quinolin-8-yl)dibenzo[b,f][1,4]oxazepin-11(10H)-one $8 \mathrm{~h}$}

8h was obtained as a yellow solid through column chromatography $(\mathrm{PE} / \mathrm{EA}=10 / 1$ to 3/1), yield $61 \%$ (45 mg).

${ }^{1} \mathrm{H}$ NMR $\left(400 \mathrm{MHz}, \mathrm{CDCl}_{3}\right) \delta_{\mathrm{H}} 9.00(1 \mathrm{H}, \mathrm{dd}, J=4.2,1.7 \mathrm{~Hz}), 8.27(1 \mathrm{H}, \mathrm{dd}, J=8.3,1.7$ Hz), $7.98(1 \mathrm{H}, \mathrm{dd}, J=8.0,1.8 \mathrm{~Hz}), 7.94(1 \mathrm{H}, \mathrm{dd}, J=8.3,1.4 \mathrm{~Hz}), 7.80(1 \mathrm{H}, \mathrm{dd}, J=7.3$, $1.4 \mathrm{~Hz}), 7.66(1 \mathrm{H}, \mathrm{dd}, J=8.2,7.3 \mathrm{~Hz}), 7.56-7.50(1 \mathrm{H}, \mathrm{m}), 7.50-7.46(1 \mathrm{H}, \mathrm{m}), 7.32-$ $7.29(2 \mathrm{H}, \mathrm{m}), 7.27(0 \mathrm{H}, \mathrm{d}, J=1.2 \mathrm{~Hz}), 6.90(1 \mathrm{H}, \mathrm{d}, J=2.8 \mathrm{~Hz}), 6.60(1 \mathrm{H}, \mathrm{d}, J=9.0$ $\mathrm{Hz}), 6.44(1 \mathrm{H}, \mathrm{dd}, J=9.0,2.8 \mathrm{~Hz}), 3.76(3 \mathrm{H}, \mathrm{s}) .{ }^{13} \mathrm{C} \mathrm{NMR}\left(125 \mathrm{MHz}, \mathrm{CDCl}_{3}\right) \delta_{\mathrm{C}}$ 
166.6, 160.4, 157.6, 154.2, 151.3, 144.9, 140.2, 136.4, 133.4, 132.5, 130.1, 129.4, 129.0, $128.7,127.2,126.9,125.4,124.9,121.8,119.9,111.3,106.5,55.6$. LRMS (ESI) $[\mathrm{M}+\mathrm{H}]^{+}$ found $\mathrm{m} / \mathrm{z} 403.3 \mathrm{HRMS}$ (ESI) $[\mathrm{M}+\mathrm{Na}]^{+}$found $\mathrm{m} / \mathrm{z} 425.0663$ calcd for $\mathrm{C}_{23} \mathrm{H}_{15} \mathrm{O}_{3} \mathrm{~N}_{2} \mathrm{ClNa}$ 425.0669 .

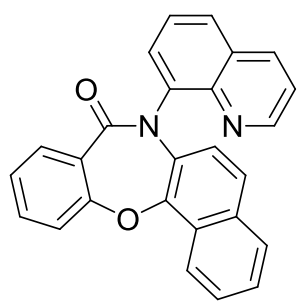

$8 \mathbf{i}$

\section{3-(quinolin-8-yl)benzo[f]naphtho[2,1-b][1,4]oxazepin-12(13H)-one 8i}

$\mathbf{8 i}$ was obtained as a yellow solid through column chromatography $(\mathrm{PE} / \mathrm{EA}=10 / 1$ to $5 / 1$ ), yield $70 \%$ (54 mg).

${ }^{1} \mathrm{H}$ NMR $\left(400 \mathrm{MHz}, \mathrm{CDCl}_{3}\right) \delta_{\mathrm{H}} 9.01(1 \mathrm{H}, \mathrm{dd}, J=4.2,1.7 \mathrm{~Hz}), 8.64(1 \mathrm{H}, \mathrm{dq}, J=8.5,0.9$ Hz), $8.30(1 \mathrm{H}, \mathrm{dd}, J=8.3,1.7 \mathrm{~Hz}), 8.10-8.00(1 \mathrm{H}, \mathrm{m}), 7.97(1 \mathrm{H}, \mathrm{dd}, J=8.3,1.4 \mathrm{~Hz})$, $7.85(1 \mathrm{H}, \mathrm{dd}, J=7.3,1.4 \mathrm{~Hz}), 7.74(1 \mathrm{H}, \mathrm{dt}, J=8.3,0.7 \mathrm{~Hz}), 7.67(2 \mathrm{H}, \mathrm{ddd}, J=8.2,7.1$, $1.2 \mathrm{~Hz}), 7.57-7.46(4 \mathrm{H}, \mathrm{m}), 7.39-7.34(1 \mathrm{H}, \mathrm{m}), 7.33-7.29(1 \mathrm{H}, \mathrm{m}), 6.82(1 \mathrm{H}, \mathrm{d}, J$ $=9.0) .{ }^{13} \mathrm{C} \mathrm{NMR}\left(125 \mathrm{MHz}, \mathrm{CDCl}_{3}\right) \delta_{\mathrm{C}} 166.9,161.0,151.5,147.7,145.1,139.8,136.4$, $133.3,132.6,131.8,131.5,130.5,129.4,129.0,127.7,127.3,127.1,126.8,126.2,125.4$, 125.0, 122.5, 121.9, 121.6, 120.1. LRMS (ESI) $[\mathrm{M}]^{+}$found $\mathrm{m} / \mathrm{z}$ 389.5. HRMS (ESI) $[\mathrm{M}+\mathrm{H}]^{+}$found $\mathrm{m} / \mathrm{z} 389.1274$, calcd for $\mathrm{C}_{26} \mathrm{H}_{17} \mathrm{O}_{2} \mathrm{~N}_{2} 389.1285$.<smiles>O=C1c2ccccc2Oc2ccc(C(F)(F)F)cc2N1c1cccc2cccnc12</smiles>

8j

\section{0-(quinolin-8-yl)-8-(trifluoromethyl)dibenzo[b,f][1,4]oxazepin-11(10H)-one 8j}

8j was obtained as a yellow solid through column chromatography (PE/EA $=10 / 1$ to $5 / 1$ ), yield $80 \%$ (65 mg). 
${ }^{1} \mathrm{H}$ NMR $\left(500 \mathrm{MHz}, \mathrm{CDCl}_{3}\right) \delta_{\mathrm{H}} 8.97(1 \mathrm{H}, \mathrm{dd}, J=4.2,1.7 \mathrm{~Hz}), 8.30(1 \mathrm{H}, \mathrm{dd}, J=8.3,1.7$ Hz), $7.99(2 \mathrm{H}, \mathrm{dt}, J=8.0,1.7 \mathrm{~Hz}), 7.84(1 \mathrm{H}, \mathrm{dd}, J=7.3,1.4 \mathrm{~Hz}), 7.70(1 \mathrm{H}, \mathrm{t}, J=7.8$ Hz), $7.56(1 \mathrm{H}, \mathrm{td}, J=7.7,1.8 \mathrm{~Hz}), 7.51(1 \mathrm{H}, \mathrm{dd}, J=8.3,4.2 \mathrm{~Hz}), 7.47(1 \mathrm{H}, \mathrm{d}, J=8.4$ $\mathrm{Hz}), 7.41-7.30(3 \mathrm{H}, \mathrm{m}), 6.93(1 \mathrm{H}, \mathrm{d}, J=2.1) .{ }^{13} \mathrm{C} \mathrm{NMR}\left(125 \mathrm{MHz}, \mathrm{CDCl}_{3}\right) \delta_{\mathrm{C}} 166.4$, $159.9,155.4,151.5,144.7,139.2,136.6,136.5,133.9,132.6,130.2,129.5,129.4,127.9$ $\left(\mathrm{q},{ }^{2} J_{\mathrm{C}-\mathrm{F}}=33.1 \mathrm{~Hz}\right), 126.8,126.7,125.9,123.3\left(\mathrm{q},{ }^{1} J_{\mathrm{C}-\mathrm{F}}=272.3 \mathrm{~Hz}\right), 122.9\left(\mathrm{q},{ }^{3} J_{\mathrm{C}-\mathrm{F}}=3.7\right.$ $\mathrm{Hz}), 122.3,122.0,121.5\left(\mathrm{q},{ }^{3} \mathrm{~J}_{\mathrm{C}-\mathrm{F}}=4.0 \mathrm{~Hz}\right), 119.8$. LRMS (ESI) $[\mathrm{M}+\mathrm{H}]^{+}$found $\mathrm{m} / \mathrm{z} 407.4$ HRMS (ESI) $[\mathrm{M}+\mathrm{H}]^{+}$found m/z 407.0999 calcd for $\mathrm{C}_{23} \mathrm{H}_{14} \mathrm{O}_{2} \mathrm{~N}_{2} \mathrm{~F}_{3} 407.1007$.

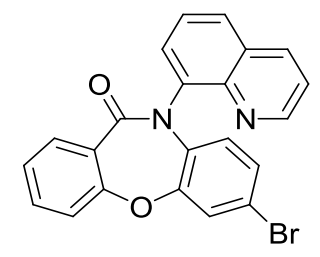

8k

\section{7-bromo-10-(quinolin-8-yl)dibenzo[b,f][1,4]oxazepin-11(10H)-one 8k}

$\mathbf{8 k}$ was obtained as a brown solid through column chromatography (PE/EA $=10 / 1$ to $5 / 1$ ), yield $72 \%(60 \mathrm{mg})$.

${ }^{1} \mathrm{H} \mathrm{NMR}\left(400 \mathrm{MHz}, \mathrm{CDCl}_{3}\right) \delta_{\mathrm{H}} 8.97(1 \mathrm{H}, \mathrm{dd}, J 4.2,1.7 \mathrm{~Hz}), 8.28(1 \mathrm{H}, \mathrm{dd}, J=8.4,1.7$ Hz), $7.97(2 \mathrm{H}, \mathrm{ddd}, J=8.3,7.4,1.6 \mathrm{~Hz}), 7.81(1 \mathrm{H}, \mathrm{dd}, J=7.3,1.4 \mathrm{~Hz}), 7.67(1 \mathrm{H}, \mathrm{dd}, J$ $=8.2,7.3 \mathrm{~Hz}), 7.58-7.54(1 \mathrm{H}, \mathrm{m}), 7.52(1 \mathrm{H}, \mathrm{d}, J=2.3 \mathrm{~Hz}), 7.50(1 \mathrm{H}, \mathrm{dd}, J=8.3,4.2$ $\mathrm{Hz}), 7.36-7.29(2 \mathrm{H}, \mathrm{m}), 7.00(1 \mathrm{H}, \mathrm{dd}, J=8.7,2.3 \mathrm{~Hz}), 6.53(1 \mathrm{H}, \mathrm{d}, J=8.7 \mathrm{~Hz}) .{ }^{13} \mathrm{C}$ NMR $\left(125 \mathrm{MHz}, \mathrm{CDCl}_{3}\right) \delta_{\mathrm{C}} 286.3,280.0,273.5,271.4,264.7,259.6,256.3,255.3,253.7$, 252.5, 250.1, 249.4, 249.0, 248.5, 246.8, 246.7, 245.7, 245.4, 244.6, 241.9, 239.8, 237.7. LRMS (ESI) $[\mathrm{M}+\mathrm{H}]^{+}$found $\mathrm{m} / \mathrm{z} 417.2 \mathrm{HRMS}(\mathrm{ESI})[\mathrm{M}+\mathrm{H}]^{+}$found $\mathrm{m} / \mathrm{z} 417.0246$ calcd for $\mathrm{C}_{22} \mathrm{H}_{14} \mathrm{O}_{2} \mathrm{~N}_{2} \mathrm{Br}$ 417.0239.<smiles>COc1ccc2c(c1)Oc1ccc(Cl)cc1NC2=O</smiles>

\section{8-chloro-3-methoxydibenzo[b,f][1,4]oxazepin-11(10H)-one 9a}


9a was obtained as a white solid through column chromatography (PE/EA = 10/1 to 5/1), yield 45\% (10mg).

${ }^{1} \mathrm{H}$ NMR $\left(400 \mathrm{MHz}, \mathrm{CDCl}_{3}\right) \delta_{\mathrm{H}} 8.71(1 \mathrm{H}, \mathrm{s}), 7.93(1 \mathrm{H}, \mathrm{d}, J=8.8 \mathrm{~Hz}), 7.20(1 \mathrm{H}, \mathrm{d}, J=9.3$ Hz), $7.13-7.06(2 \mathrm{H}, \mathrm{m}), 6.82(1 \mathrm{H}, \mathrm{dd}, J=8.8,2.5 \mathrm{~Hz}), 6.74(1 \mathrm{H}, \mathrm{d}, J=2.5 \mathrm{~Hz}), 3.89(3 \mathrm{H}$, s). ${ }^{13} \mathrm{C}$ NMR $\left(125 \mathrm{MHz}, \mathrm{CDCl}_{3}\right) \delta_{\mathrm{C}} 166.8,164.9,160.6,148.9,133.5,131.9,130.9,125.3$, 122.8, 121.0, 116.8, 111.7, 105.6, 55.8. LRMS (EI) $[\mathrm{M}]^{+}$found $\mathrm{m} / \mathrm{z} 275$. HRMS (EI) $[\mathrm{M}]^{+}$found $\mathrm{m} / \mathrm{z} 275.0355$, calcd for $\mathrm{C}_{14} \mathrm{H}_{10} \mathrm{O}_{3} \mathrm{NCl} 275.0349$.<smiles>Cc1ccc2c(c1)Oc1ccc(Cl)cc1NC2=O</smiles>

\section{8-chloro-3-methyldibenzo[b,f][1,4]oxazepin-11(10H)-one 9b}

9b was obtained as a white solid through column chromatography (PE/EA $=10 / 1$ to $5 / 1)$, yield $49 \%$ (10mg).

${ }^{1} \mathrm{H}$ NMR $\left(400 \mathrm{MHz}, \mathrm{CDCl}_{3}\right) \delta_{\mathrm{H}} 8.35(1 \mathrm{H}, \mathrm{s}), 7.85(1 \mathrm{H}, \mathrm{d}, J=7.9 \mathrm{~Hz}), 7.20(1 \mathrm{H}, \mathrm{d}, J=$ $8.5 \mathrm{~Hz}), 7.12-7.05(3 \mathrm{H}, \mathrm{m}), 2.42(3 \mathrm{H}, \mathrm{d}, J=0.8 \mathrm{~Hz}) .166 .8,159.1,149.4,146.2,132.0$, $131.7,130.8,126.4,125.5,122.8,121.6,121.1,120.9,21.4 .{ }^{13} \mathrm{C}$ NMR $\left(125 \mathrm{MHz}, \mathrm{CDCl}_{3}\right)$ $\delta_{\mathrm{C}} 166.8,159.1,149.4,146.2,132.0,131.7,130.8,126.4,125.5,122.8,121.6,121.1,120.9$, 21.4. LRMS (EI) $[\mathrm{M}]^{+}$found $\mathrm{m} / \mathrm{z}$ 259. HRMS (EI) $[\mathrm{M}]^{+}$found $\mathrm{m} / \mathrm{z} 259.0399$, calcd for $\mathrm{C}_{14} \mathrm{H}_{10} \mathrm{O}_{2} \mathrm{NCl} 259.0400$<smiles>O=C1Nc2cc(Cl)ccc2Oc2ccccc21</smiles>

\section{8-chlorodibenzo[b,f][1,4]oxazepin-11(10H)-one 9c}

9c was obtained as a brown solid through column chromatography $(\mathrm{PE} / \mathrm{EA}=10 / 1$ to 5/1), yield $50 \%(11 \mathrm{mg})$.

${ }^{1} \mathrm{H}$ NMR (400 MHz, DMSO- $\left.d_{6}\right) \delta_{\mathrm{H}} 10.64(1 \mathrm{H}, \mathrm{s}), 7.78(1 \mathrm{H}, \mathrm{dd}, J=7.7,1.8 \mathrm{~Hz}), 7.69-$ $7.58(1 \mathrm{H}, \mathrm{m}), 7.42-7.30(3 \mathrm{H}, \mathrm{m}), 7.24-7.17(2 \mathrm{H}, \mathrm{m}) .{ }^{13} \mathrm{C}\left(125 \mathrm{MHz}, \mathrm{DMSO}-d_{6}\right) \delta_{\mathrm{C}}$ 
$166.0,158.9,149.5,135.2,133.1,131.9,129.9,126.2,125.8,125.2,123.5,121.4,121.1$. LRMS (EI) $[\mathrm{M}]^{+}$found $\mathrm{m} / \mathrm{z}$ 245. HRMS (EI) $[\mathrm{M}]^{+}$found $\mathrm{m} / \mathrm{z}$ 245.0241, calcd for $\mathrm{C}_{13} \mathrm{H}_{8} \mathrm{O}_{2} \mathrm{NCl} 245.0243$.<smiles>O=C1Nc2ccc(C(F)(F)F)cc2Oc2ccccc21</smiles>

$9 d$

\section{7-(trifluoromethyl)dibenzo[b,f][1,4]oxazepin-11(10H)-one 9d}

9c was obtained as a brown solid through column chromatography (PE/EA = 10/1 to 5/1), yield 55\% (12 mg).

${ }^{1} \mathrm{H}$ NMR $\left(400 \mathrm{MHz}, \mathrm{CDCl}_{3}\right) \delta_{\mathrm{H}} 9.08(1 \mathrm{H}, \mathrm{s}), 8.08-7.91(1 \mathrm{H}, \mathrm{m}), 7.60(1 \mathrm{H}, \mathrm{ddd}, J=$ 8.0, 7.4, $1.8 \mathrm{~Hz}), 7.57(1 \mathrm{H}, \mathrm{d}, J=2.0 \mathrm{~Hz}), 7.45(1 \mathrm{H}, \mathrm{ddd}, J=8.3,2.0,0.8 \mathrm{~Hz}), 7.36-$ $7.29(2 \mathrm{H}, \mathrm{m}), 7.24(1 \mathrm{H}, \mathrm{dd}, J=8.3,0.9 \mathrm{~Hz}) .{ }^{13} \mathrm{C} \mathrm{NMR}\left(125 \mathrm{MHz}, \mathrm{CDCl}_{3}\right) \delta_{\mathrm{C}} 167.1$, $159.0,150.3,135.0,133.9,132.2,128.0\left(\mathrm{q},{ }^{2} J_{\mathrm{C}-\mathrm{F}}=33.5 \mathrm{~Hz}\right), 125.7,124.5,123.4\left(\mathrm{q},{ }^{1} J_{\mathrm{C}-\mathrm{F}}\right.$ $=272.1 \mathrm{~Hz}), 123.0\left(\mathrm{q},{ }^{3} J_{\mathrm{C}-\mathrm{F}}=3.9 \mathrm{~Hz}\right), 121.4,120.9,119.4\left(\mathrm{q},{ }^{3} J_{\mathrm{C}-\mathrm{F}}=3.9 \mathrm{~Hz}\right)$. LRMS (EI) $[\mathrm{M}]^{+}$found $\mathrm{m} / \mathrm{z}$ 279. HRMS (EI) $[\mathrm{M}]^{+}$found $\mathrm{m} / \mathrm{z}$ 279.0507, calcd for $\mathrm{C}_{14} \mathrm{H}_{8} \mathrm{O}_{2} \mathrm{NF}_{3}$ 279.0507 .

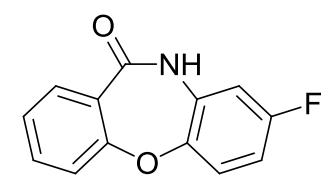

$9 e$

\section{8-fluorodibenzo[b,f][1,4]oxazepin-11(10H)-one 9e}

9e was obtained as a white solid through column chromatography (PE/EA $=10 / 1$ to $5 / 1)$, yield 58\% (11 mg).

${ }^{1} \mathrm{H}$ NMR $\left(400 \mathrm{MHz}, \mathrm{CDCl}_{3}\right) \delta_{\mathrm{H}} 9.1(1 \mathrm{H}, \mathrm{s}), 8.0(1 \mathrm{H}, \mathrm{dd}, J=7.8,1.8 \mathrm{~Hz}), 7.5(1 \mathrm{H}, \mathrm{ddd}, J=$ 8.0, 7.3, 1.8 Hz), $7.3-7.3(1 \mathrm{H}, \mathrm{m}), 7.3-7.2(2 \mathrm{H}, \mathrm{m}), 6.9-6.8(2 \mathrm{H}, \mathrm{m}) .{ }^{13} \mathrm{C}$ NMR $(125$ $\left.\mathrm{MHz}, \mathrm{CDCl}_{3}\right) \delta_{\mathrm{C}} 167.7,159.8\left(\mathrm{~d},{ }^{1} J_{\mathrm{C}-\mathrm{F}}=244.5 \mathrm{~Hz}\right), 159.6,147.0\left(\mathrm{~d},{ }^{4} J_{\mathrm{C}-\mathrm{F}}=2.7 \mathrm{~Hz}\right), 131.7$ $\left(\mathrm{d},{ }^{3} J=10.7 \mathrm{~Hz}\right), 134.8,132.1,125.4,124.9,122.6\left(\mathrm{~d},{ }^{3} J=9.6 \mathrm{~Hz}\right), 120.7,112.1\left(\mathrm{~d},{ }^{2} J=23.1\right.$ $\mathrm{Hz}), 108.3\left(\mathrm{~d},{ }^{2} J=26.3 \mathrm{~Hz}\right.$ ). LRMS (EI) $[\mathrm{M}]^{+}$found $\mathrm{m} / \mathrm{z} 229$. HRMS (EI) $[\mathrm{M}]^{+}$found $\mathrm{m} / \mathrm{z}$ 229.0539, calcd for $\mathrm{C}_{13} \mathrm{H}_{8} \mathrm{O}_{2} \mathrm{NF} 229.0539$. 
<smiles>O=C(O)c1ccccc1Oc1ccc(Cl)cc1Br</smiles>

10

2-(2-bromo-4-chlorophenoxy)benzoic acid 10

10 was obtained as a brown solid through column chromatography $(\mathrm{PE} / \mathrm{EA}=10 / 1$ to1/1), yield 45\% (300 mg).

${ }^{1} \mathrm{H}$ NMR $\left(400 \mathrm{MHz}, \mathrm{CDCl}_{3}\right) \delta_{\mathrm{H}} 8.22-8.06(1 \mathrm{H}, \mathrm{m}), 7.67(1 \mathrm{H}, \mathrm{d}, J=2.5 \mathrm{~Hz}), 7.50(1 \mathrm{H}$, td, $J=8.7,8.0,1.7 \mathrm{~Hz}), 7.30(1 \mathrm{H}, \mathrm{d}, J=2.5 \mathrm{~Hz}), 7.27(0 \mathrm{H}, \mathrm{d}, J=2.5 \mathrm{~Hz}), 7.27-7.21$ $(1 \mathrm{H}, \mathrm{m}), 6.94(1 \mathrm{H}, \mathrm{d}, J=8.7 \mathrm{~Hz}), 6.79(1 \mathrm{H}, \mathrm{dd}, J=8.3,1.0 \mathrm{~Hz}) \cdot{ }^{13} \mathrm{C}$ NMR $(125 \mathrm{MHz}$, $\left.\mathrm{CDCl}_{3}\right) \delta_{\mathrm{C}} 287.8,276.0,271.4,254.5,253.5,253.3,250.4,248.9,244.0,241.5,240.8$, 238.0, 235.5.. LRMS (ESI) $[\mathrm{M}+\mathrm{H}]^{+}$found $\mathrm{m} / \mathrm{z}$ 326.8. HRMS (ESI) $[\mathrm{M}-\mathrm{H}]-$ found $\mathrm{m} / \mathrm{z}$ 324.9275, calcd for $\mathrm{C}_{13} \mathrm{H}_{7} \mathrm{O}_{3} \mathrm{ClBr} 324.9267$.<smiles>[M]Oc1ccc(Oc2ccccc2C(=O)Nc2cccc3cccnc23)c(Br)c1</smiles>

2-(2-bromo-4-chlorophenoxy)-N-(quinolin-8-yl)benzamide M1

M1 was obtained as a yellow oil through column chromatography (PE/EA $=20 / 1$ to10/1), yield $45 \%$ (25 mg).

${ }^{1} \mathrm{H}$ NMR $\left(400 \mathrm{MHz}\right.$, Acetone- $\left.d_{6}\right) \delta_{\mathrm{H}} 12.06(1 \mathrm{H}, \mathrm{s}), 9.00(1 \mathrm{H}, \mathrm{dd}, J=7.2,1.8 \mathrm{~Hz}), 8.62$ $(1 \mathrm{H}, \mathrm{dd}, J=4.2,1.7 \mathrm{~Hz}), 8.36(2 \mathrm{H}, \mathrm{dt}, J=7.8,1.5 \mathrm{~Hz}), 7.92(1 \mathrm{H}, \mathrm{d}, J=2.5 \mathrm{~Hz}), 7.73-$ $7.52(5 \mathrm{H}, \mathrm{m}), 7.43(1 \mathrm{H}, \mathrm{d}, J=8.7 \mathrm{~Hz}), 7.39(1 \mathrm{H}, \mathrm{ddd}, J=7.7,7.2,1.1 \mathrm{~Hz}), 6.94(1 \mathrm{H}$, $\mathrm{dd}, J=8.3,1.0 \mathrm{~Hz}) .{ }^{13} \mathrm{C}$ NMR $\left(125 \mathrm{MHz}\right.$, Acetone- $\left.d_{6}\right) \delta_{\mathrm{C}} 162.7,155.6,151.9,149.0$, $139.3,136.8,136.0,134.0,133.8,132.8,131.1,130.1,128.7,127.7,124.9,124.5,124.0$, 122.5, 122.3, 117.5, 117.0, 116.7. LRMS (EI) $[\mathrm{M}]^{+}$found $\mathrm{m} / \mathrm{z}$ 451. HRMS (EI) $[\mathrm{M}]^{+}$ 
found $\mathrm{m} / \mathrm{z} 451.9933$, calcd for $\mathrm{C}_{22} \mathrm{H}_{14} \mathrm{O}_{2} \mathrm{~N}_{2} \mathrm{ClBr} 451.9927$.<smiles>CCNC(=O)c1ccccc1Oc1ccc(Cl)cc1Br</smiles>

2-(2-bromo-4-chlorophenoxy)-N-ethylbenzamide M2

M2 was obtained as a colourless oil through column chromatography (PE:EA $=9 / 1$ to $2 / 1)$, yield $62 \%(0.79 \mathrm{~g})$.

${ }^{1} \mathrm{H}$ NMR $\left(400 \mathrm{MHz}, \mathrm{CDCl}_{3}\right) \delta_{\mathrm{H}} 7.86(1 \mathrm{H}, \mathrm{dd}, J 7.8,1.7), 7.50-7.41(1 \mathrm{H}, \mathrm{m}), 7.29(1 \mathrm{H}$, t, J 2.5), $7.25(2 \mathrm{H}, \mathrm{dt}, J$ 7.6, 3.1), $7.18(2 \mathrm{H}, \mathrm{dt}, J$ 8.8, 2.0), $4.15(2 \mathrm{H}, \mathrm{q}, J$ 7.5), 1.37 (3 H, t, $J$ 7.1). ${ }^{13} \mathrm{C}$ NMR $\left(125 \mathrm{MHz}, \mathrm{CDCl}_{3}\right) \delta_{\mathrm{C}} 164.3,153.5,151.2,133.5,132.5,132.5,130.3$, $129.1,124.8,124.6,120.6,117.7,114.8,34.9,14.7$. LRMS (ESI) $[\mathrm{M}+\mathrm{Na}]^{+}$found $\mathrm{m} / \mathrm{z}$ 376.0. HRMS (ESI) $[\mathrm{M}+\mathrm{Na}]^{+}$found $\mathrm{m} / \mathrm{z}$ 375.9722, calcd for $\mathrm{C}_{15} \mathrm{H}_{13} \mathrm{NaO}_{2} \mathrm{ClBr} 375.9716$.<smiles>CCNC(=O)c1ccccc1I</smiles>

\section{N-ethyl-2-iodobenzamide 3}

3 was obtained as a white solid through column chromatography (PE/EA $=5 / 1$ to 3/1), yield $70 \%(1 \mathrm{~g})$.

${ }^{1} \mathrm{H}$ NMR $\left(400 \mathrm{MHz}, \mathrm{CDCl}_{3}\right) \delta_{\mathrm{H}} 7.91-7.70(1 \mathrm{H}, \mathrm{m}), 7.37-7.23(2 \mathrm{H}, \mathrm{m}), 7.08-6.98(1 \mathrm{H}$, m), $6.21(1 \mathrm{H}, \mathrm{s}), 3.39\left(2 \mathrm{H}, \mathrm{qd}, J\right.$ 7.2, 5.6), $1.20\left(3 \mathrm{H}, \mathrm{t}, J\right.$ 7.3). LRMS (ESI) $[\mathrm{M}+\mathrm{H}]^{+}$found $\mathrm{m} / \mathrm{z} 276.2$.

Spectral data were consistent with the literature ${ }^{2}$. 


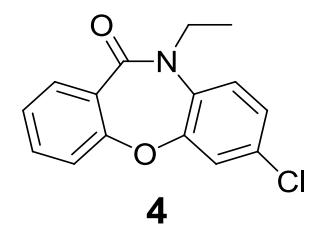

\section{7-chloro-10-ethyldibenzo[b,f][1,4]oxazepin-11(10H)-one 4}

4 was obtained as a yellow oil through column chromatography (PE/EA =10/1 to 4/1), yield $95 \%(0.26 \mathrm{~g})$.

${ }^{1} \mathrm{H}$ NMR $\left(400 \mathrm{MHz}, \mathrm{CDCl}_{3}\right) \delta_{\mathrm{H}} 7.86(1 \mathrm{H}, \mathrm{dd}, J 7.8,1.7), 7.50-7.41(1 \mathrm{H}, \mathrm{m}), 7.29(1 \mathrm{H}, \mathrm{t}$, $J$ 2.5), $7.25(2 \mathrm{H}, \mathrm{dt}, J$ 7.6, 3.1), $7.18(2 \mathrm{H}, \mathrm{dt}, J 8.8,2.0), 4.15(2 \mathrm{H}, \mathrm{q}, J 7.5), 1.37(3 \mathrm{H}, \mathrm{t}, J$ 7.1). LRMS (ESI) $[\mathrm{M}+\mathrm{H}]^{+}$found $\mathrm{m} / \mathrm{z} 274.2$.

Spectral data were consistent with the literature ${ }^{2}$.

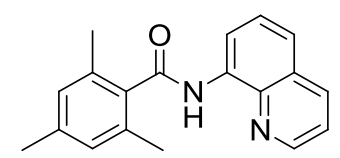

11

\section{2,4,6-trimethyl-N-(quinolin-8-yl)benzamide 11}

11 was obtained as a white soil through column chromatography (PE/EA =20/1 to 10/1), yield $80 \%(1.2 \mathrm{~g})$.

${ }^{1} \mathrm{H}$ NMR $\left(400 \mathrm{MHz}, \mathrm{CDCl}_{3}\right) \delta_{\mathrm{H}} 9.95(1 \mathrm{H}, \mathrm{s}), 9.02(1 \mathrm{H}, \mathrm{dd}, J 4.7,2.9), 8.76(1 \mathrm{H}, \mathrm{dd}, J 4.2$, 1.7), 8.20 (1 H, dt, J 8.4, 1.6), 7.73 - $7.55(2 \mathrm{H}, \mathrm{m}), 7.47$ (1 H, ddd, J 8.3, 4.2, 1.2), 6.96 (2 H, s), $2.43(6 \mathrm{H}, \mathrm{d}), 2.36(3 \mathrm{H}, \mathrm{s})$. LRMS (ESI) $[\mathrm{M}+\mathrm{H}]^{+}$found m/z 291.2.

Spectral data were consistent with the literature ${ }^{4}$. 
(5) The X-ray Crystallography Data of 7a and 8a

\subsection{The X-ray crystallography data of 7a}

Cell: $\quad a=9.9210(9) \AA ̊ \quad b=17.9238(15) \AA) c=12.9555(11) \AA$

alpha $=90^{\circ}$ beta $=90.022^{\circ}$ gamma $=90^{\circ}$

$\mathrm{Z}=4$.

Temperature: $296 \mathrm{~K}$

$\begin{array}{lcc} & \text { Calculated } & \text { Reported } \\ \text { Volume } & 2303.8(3) \AA^{3} & 2303.8(3) \AA^{3} \\ \text { Space group } & \mathrm{P} 121 / \mathrm{c} 1 & \mathrm{P} 121 / \mathrm{c} 1 \\ \text { Moiety formula } & \mathrm{C}_{22} \mathrm{H}_{14} \mathrm{ClN}_{2} \mathrm{O}_{2},\left(\mathrm{C}_{4} \mathrm{H}_{8} \mathrm{O}_{2}, \mathrm{Cl}\right) & \\ \text { Sum formula } & \mathrm{C}_{26} \mathrm{H}_{22} \mathrm{Cl}_{2} \mathrm{~N}_{2} \mathrm{O}_{4} & \mathrm{C}_{26} \mathrm{H}_{22} \mathrm{Cl}_{2} \mathrm{~N}_{2} \mathrm{O}_{4}\end{array}$

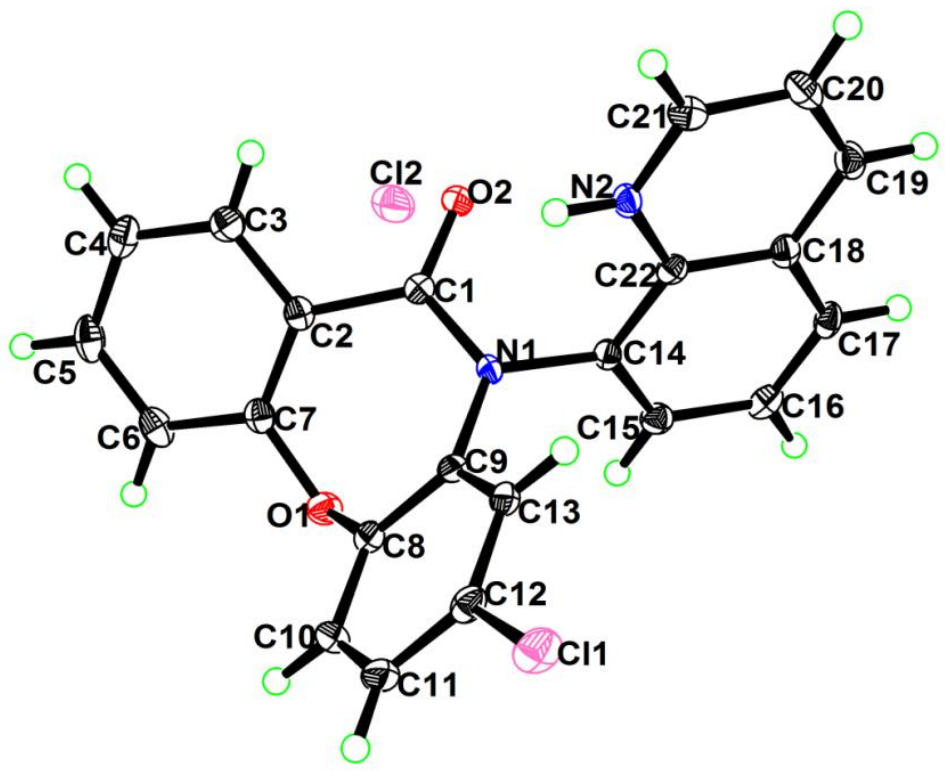

Figure S1. The crystal structure of 7a by X-ray analysis.

These data can be obtained free of charge from the Cambridge Crystallographic The CCDC number is 1435194. 


\subsection{The X-ray crystallography data of 8a}

Cell: $\quad a=14.8316 \AA$ (3) $b=16.4395 \AA$ (3) c=7.8011(2) alpha $=90^{\circ}$ beta $=93.277^{\circ}(1)$ gamma $=90^{\circ}$ $\mathrm{Z}=4$

Temperature: $170 \mathrm{~K}$

Calculated

Volume

Space group

Moiety formula

Sum formula
1898.99(7) $\AA^{3}$

$\mathrm{P} 121 / \mathrm{c} 1$

$\mathrm{C}_{22} \mathrm{H}_{13} \mathrm{ClN}_{2} \mathrm{O}_{2}, 0.5(\mathrm{C} 4 \mathrm{H} 8 \mathrm{O} 2)$

$\mathrm{C}_{24} \mathrm{H}_{17} \mathrm{Cl} \mathrm{N}_{2} \mathrm{O}_{3}$
Reported

1898.99(7) $\AA^{3}$

P $121 /$ c 1

$\mathrm{C}_{24} \mathrm{H}_{17} \mathrm{ClN}_{2} \mathrm{O}_{3}$

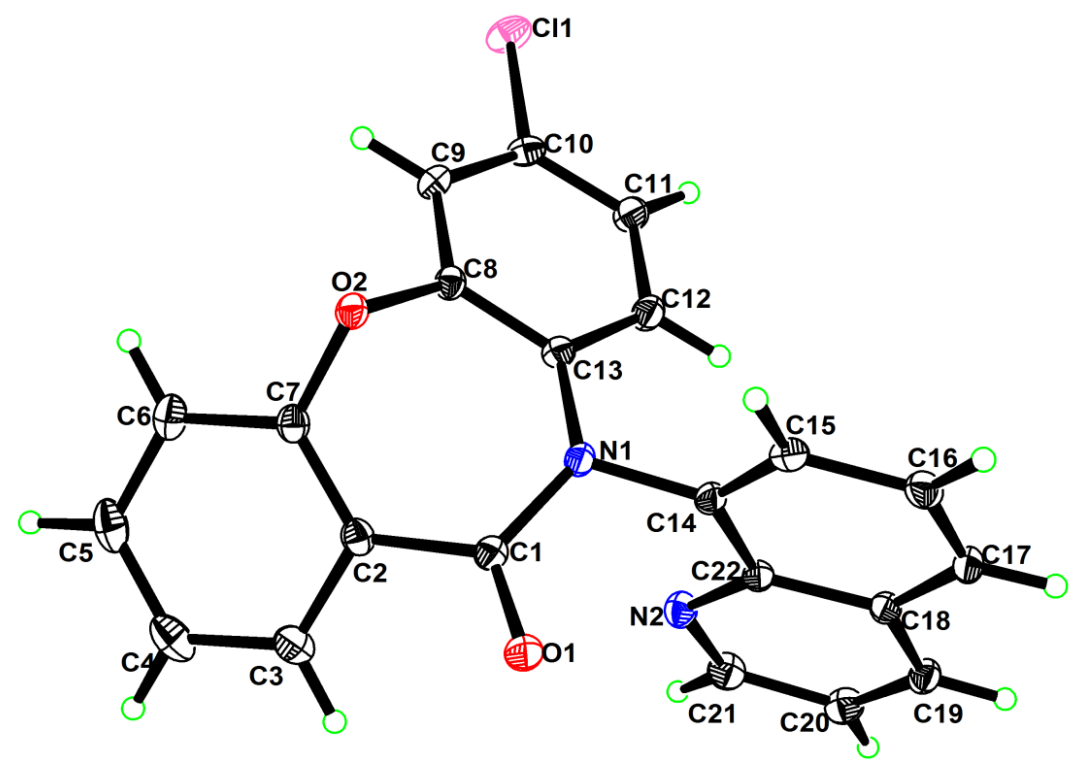

Figure S2. The crystal structure of $\mathbf{8 a}$ by X-ray analysis.

These data can be obtained free of charge from the Cambridge Crystallographic The CCDC number is 1435240 


\section{(6) Reference}

1. Tran, L. D.; Roane, J.; Daugulis, O. Angew. Chem., Int. Ed. 2013, 52, 6043.

2. Kitching, M. O.; Hurst, T. E.;Snieckus, V. Angew. Chem., Int. Ed. 2012, 51, 2925.

3. He, G.; Zhang, S.-Y.; Nack, W. A.; Li, Q.; Chen, G. Angew. Chem., Int. Ed. 2013, 52, 11124.

4. Zhang, M. Journal of Chemical Research. 2013, 37, 606. 
(7) Copies of ${ }^{1} \mathrm{H}$ NMR and ${ }^{13} \mathrm{C}$ NMR Spectra for the Products 

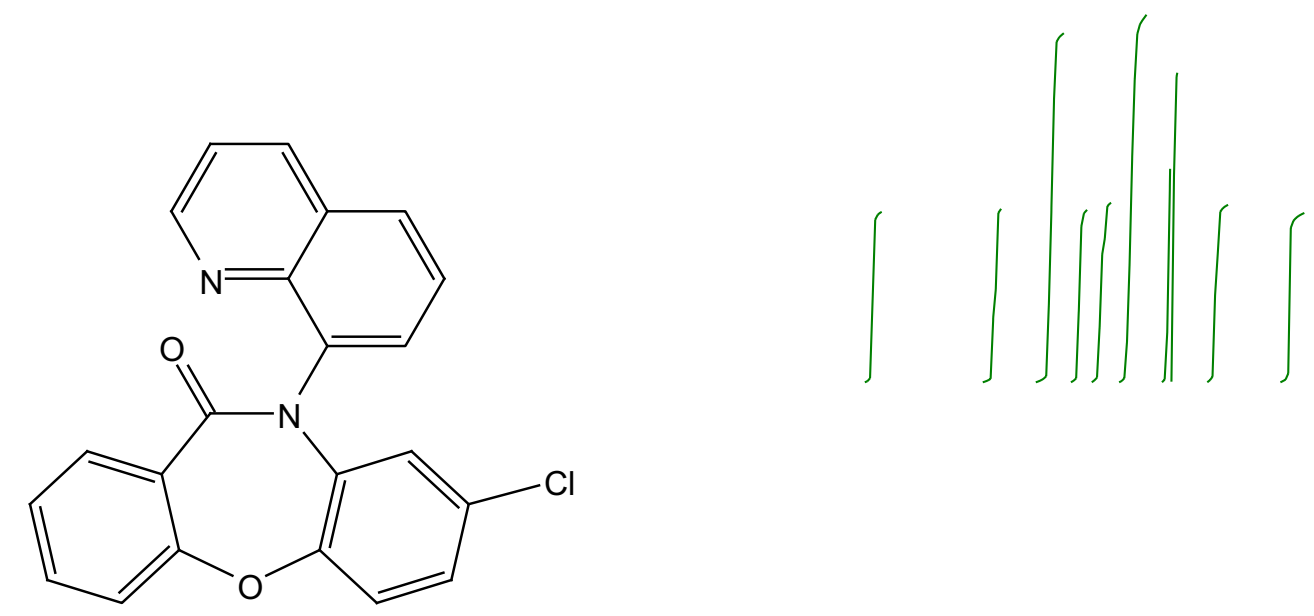

$7 a$

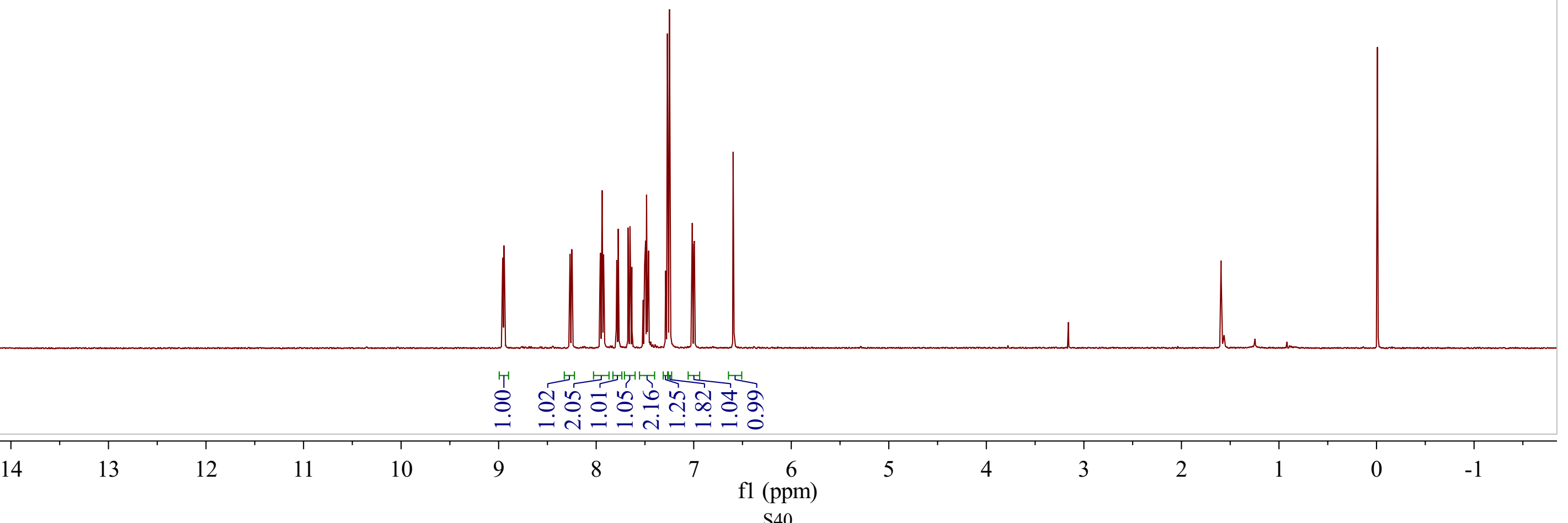




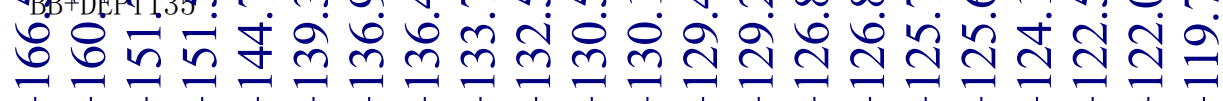
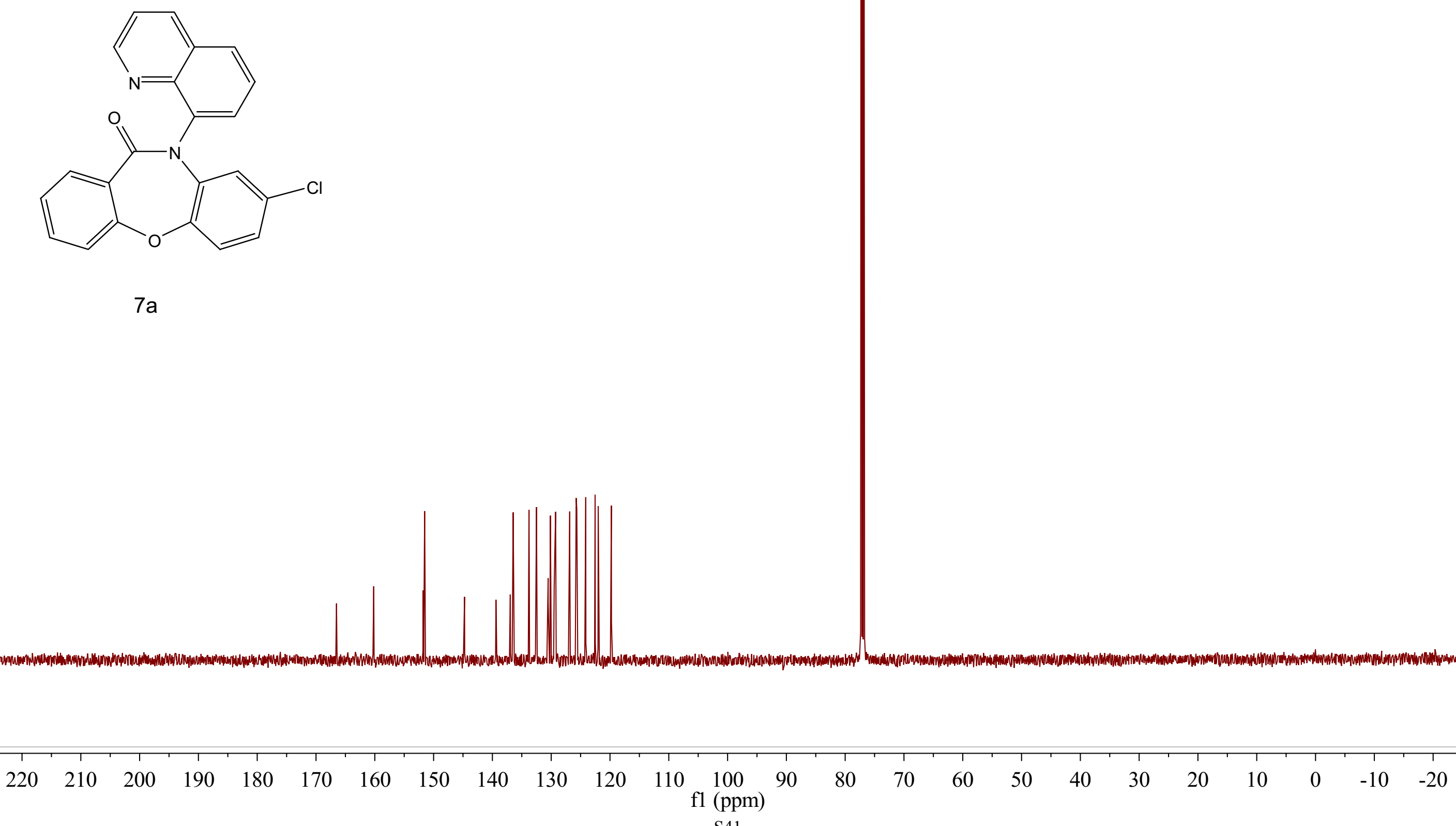


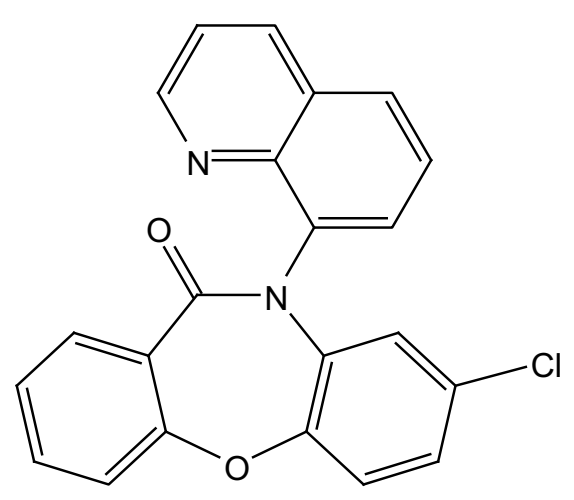

$7 a$

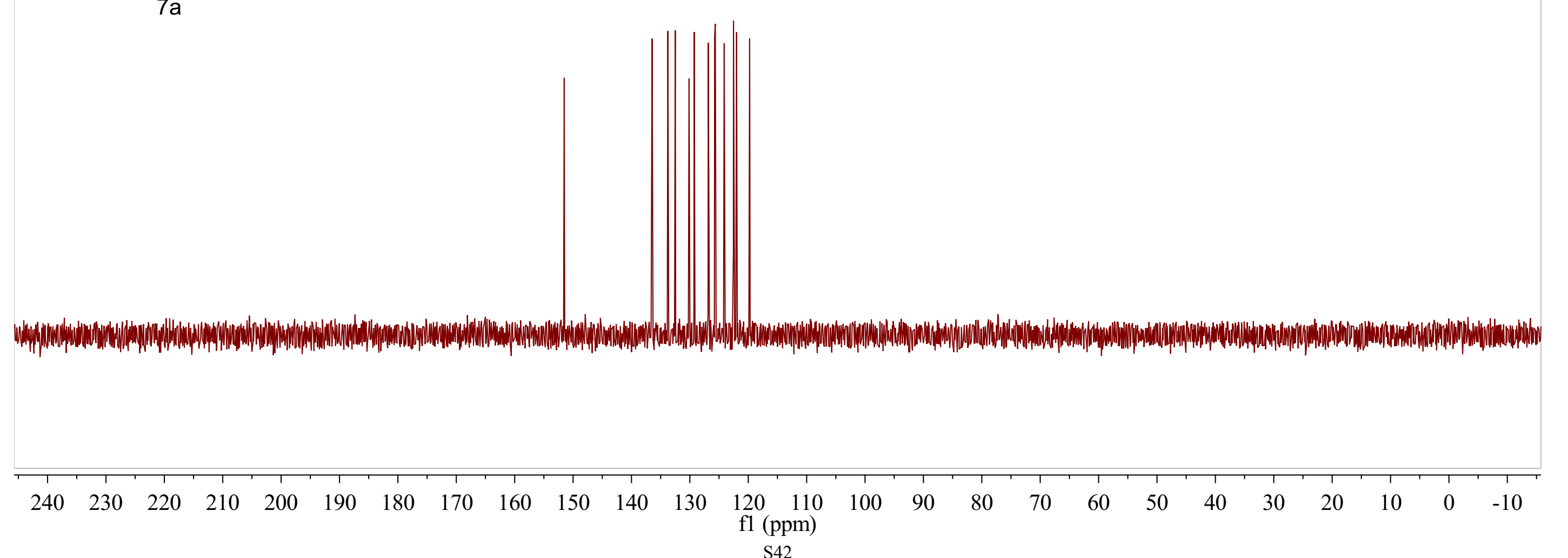


$7 b$
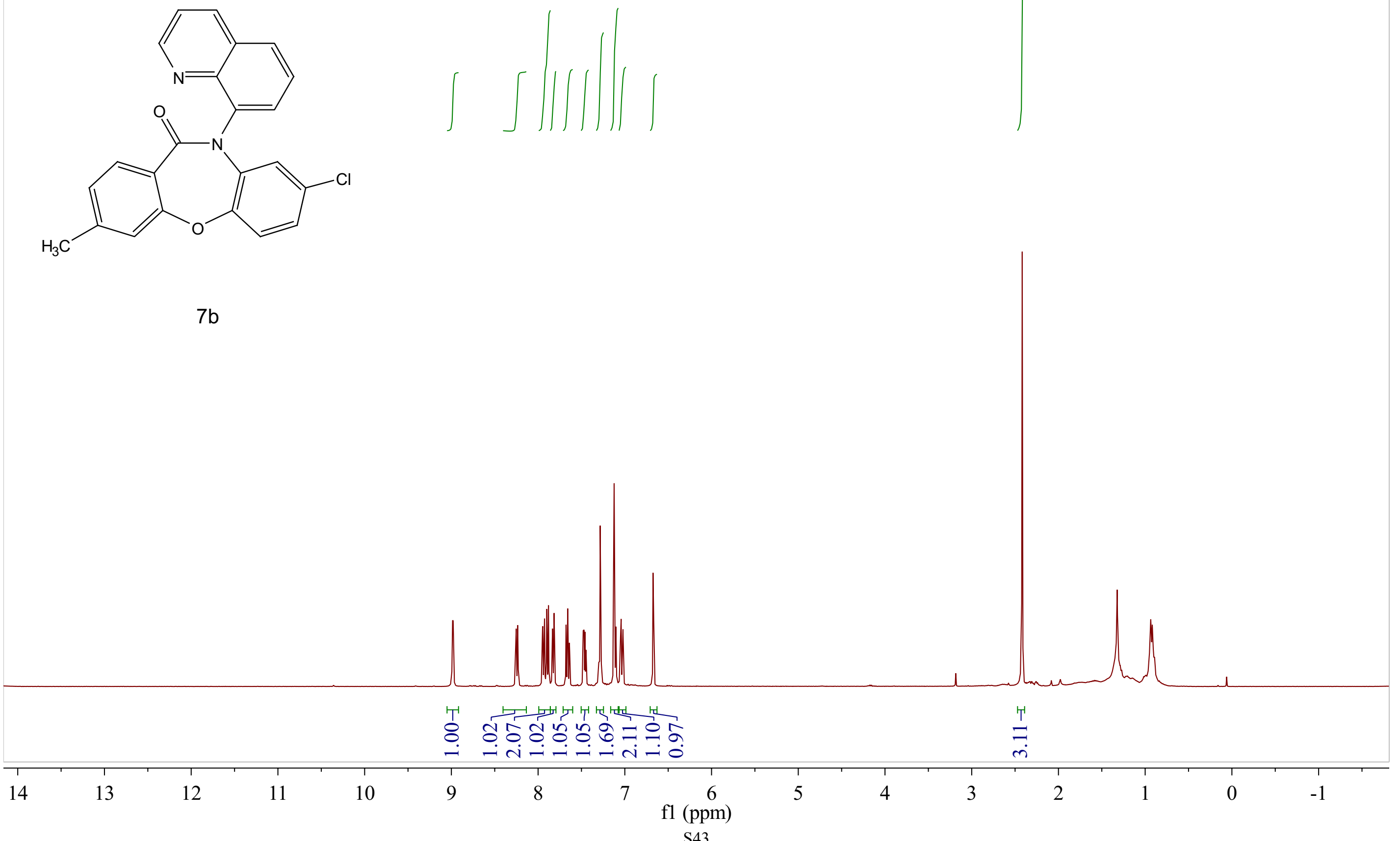


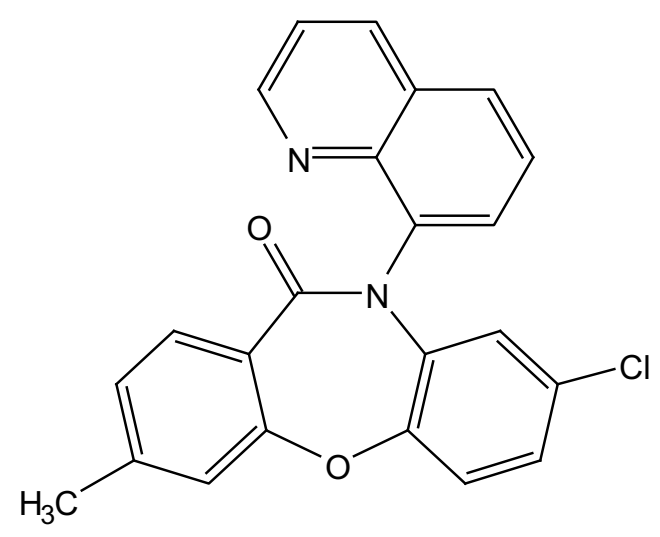

$7 b$

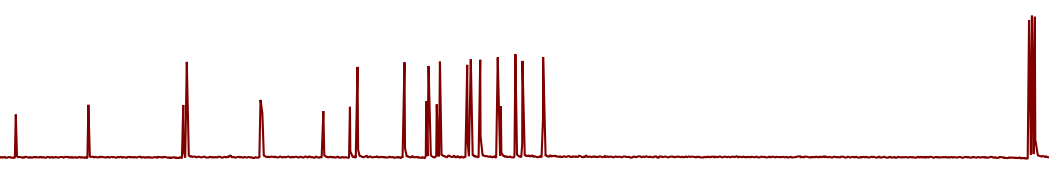

$\begin{array}{rllllllllllllllllllllllll}230 & 220 & 210 & 200 & 190 & 180 & 170 & 160 & 150 & 140 & 130 & 120 & 110 & 100 & 90 & 80 & 70 & 60 & 50 & 40 & 30 & 20 & 10 & 0 & -10\end{array}$




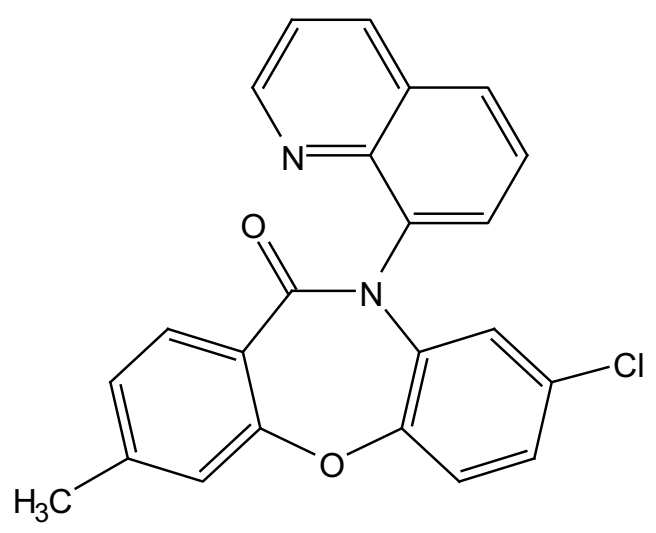

$7 b$

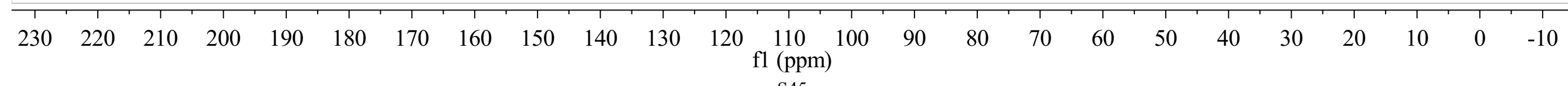




\section{8}

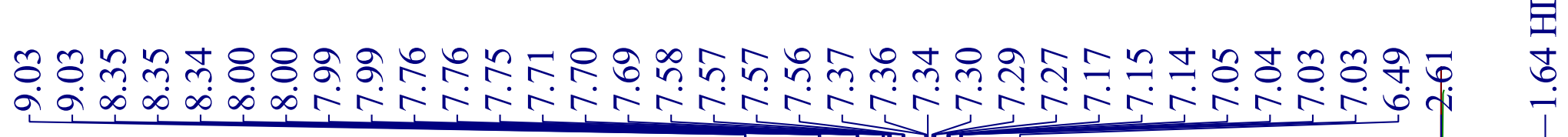
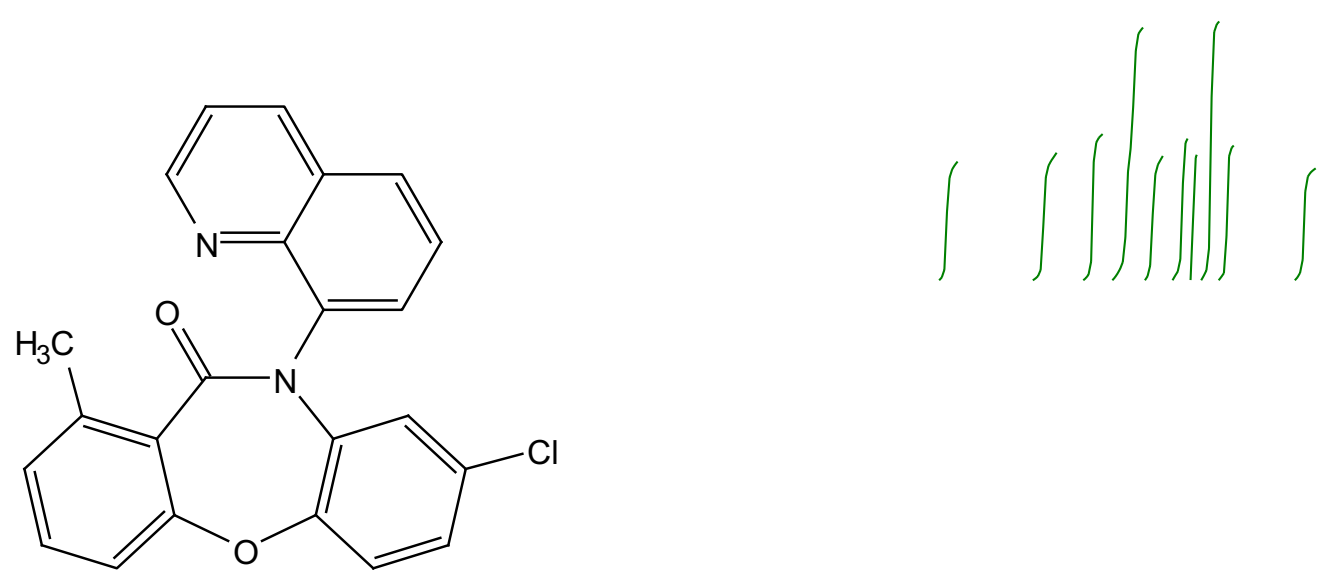

$7 \mathrm{c}$

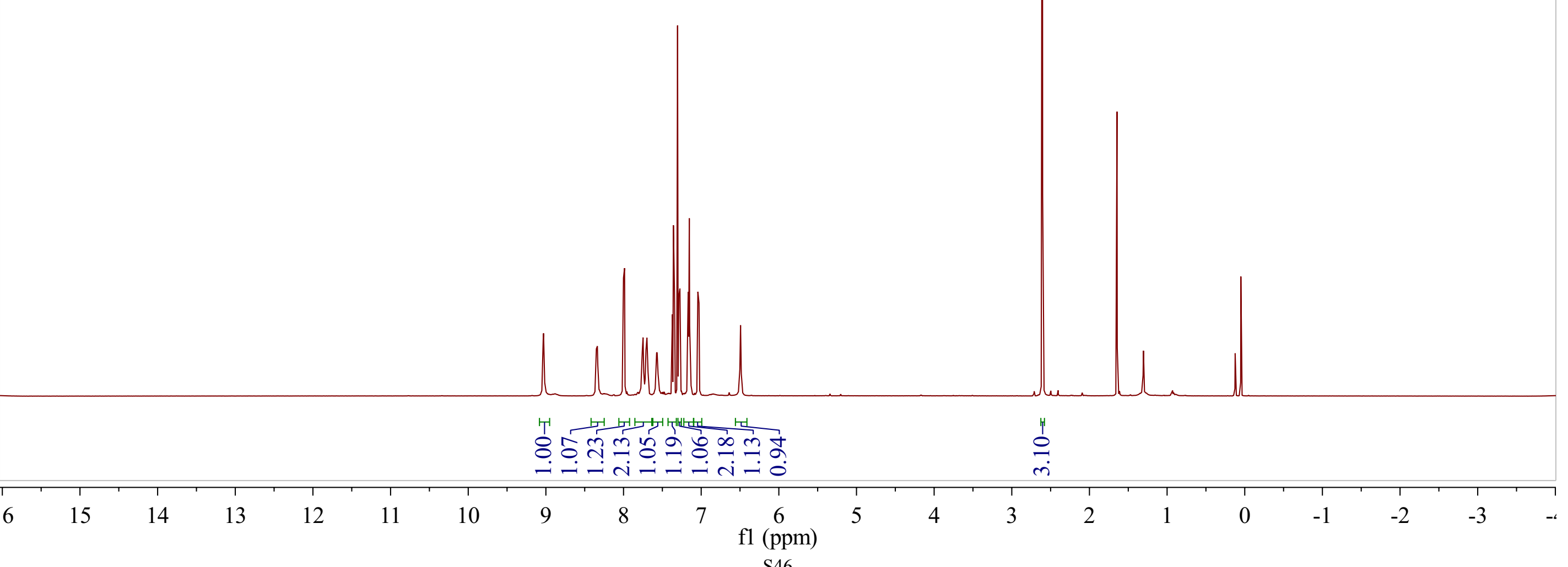




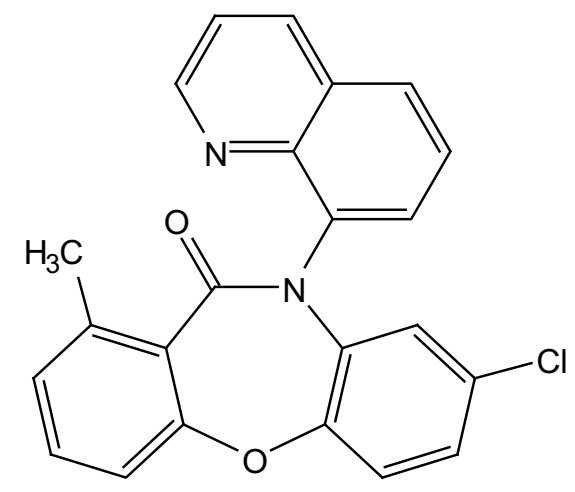

7c

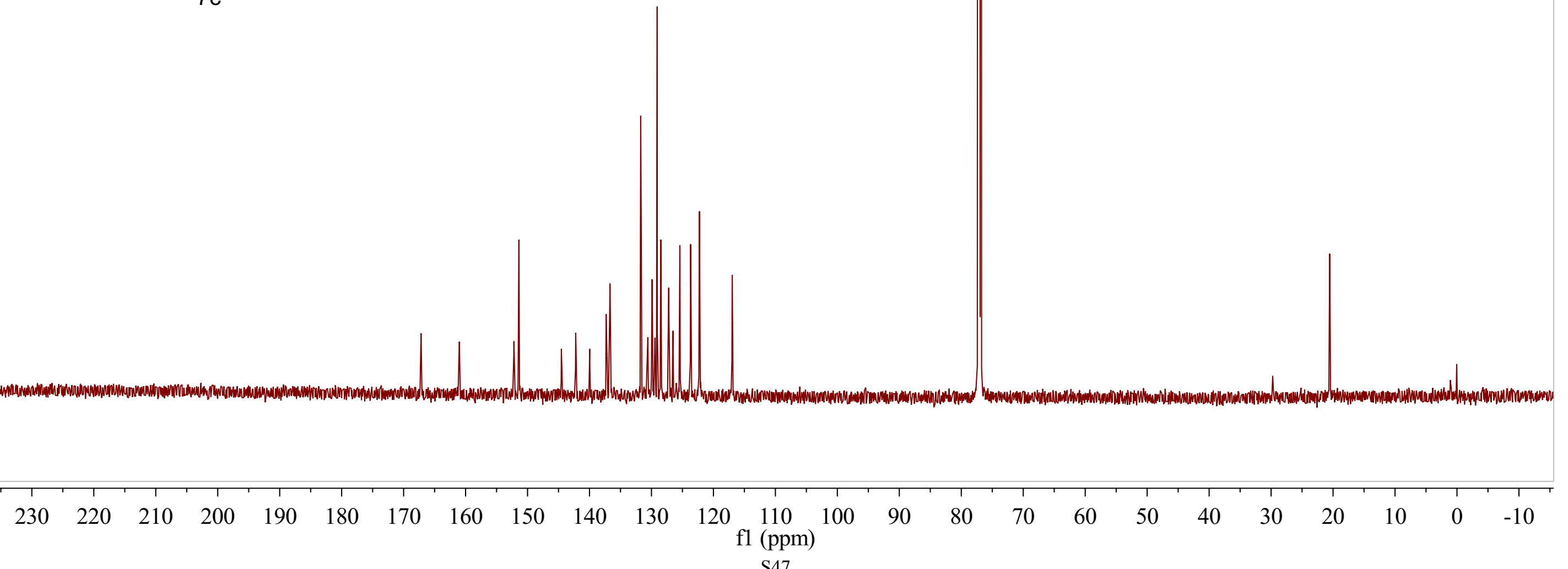




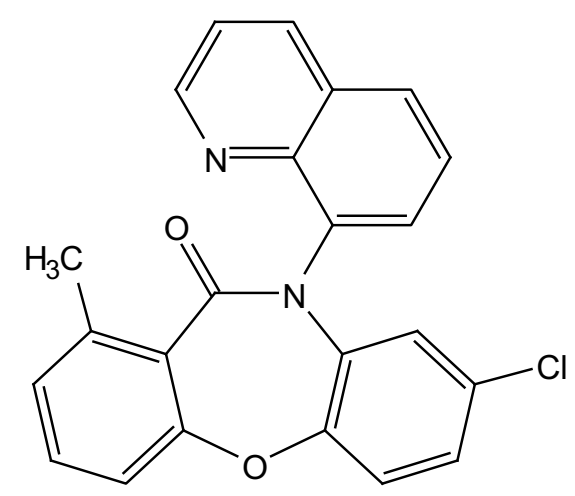

7c$$
\text { (c) }
$$$$
\text { I }
$$

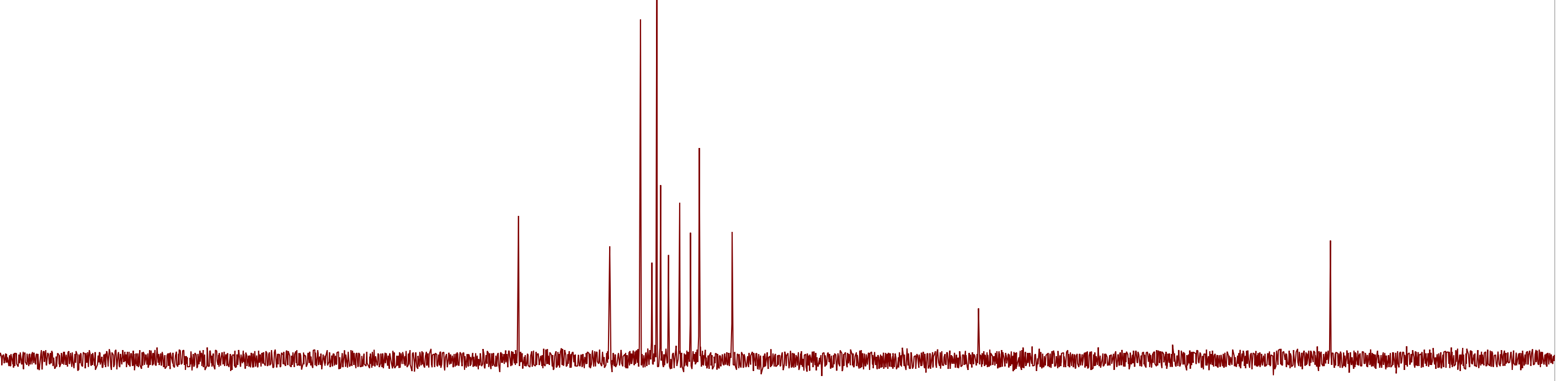

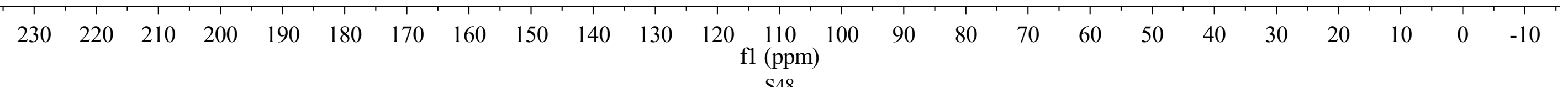



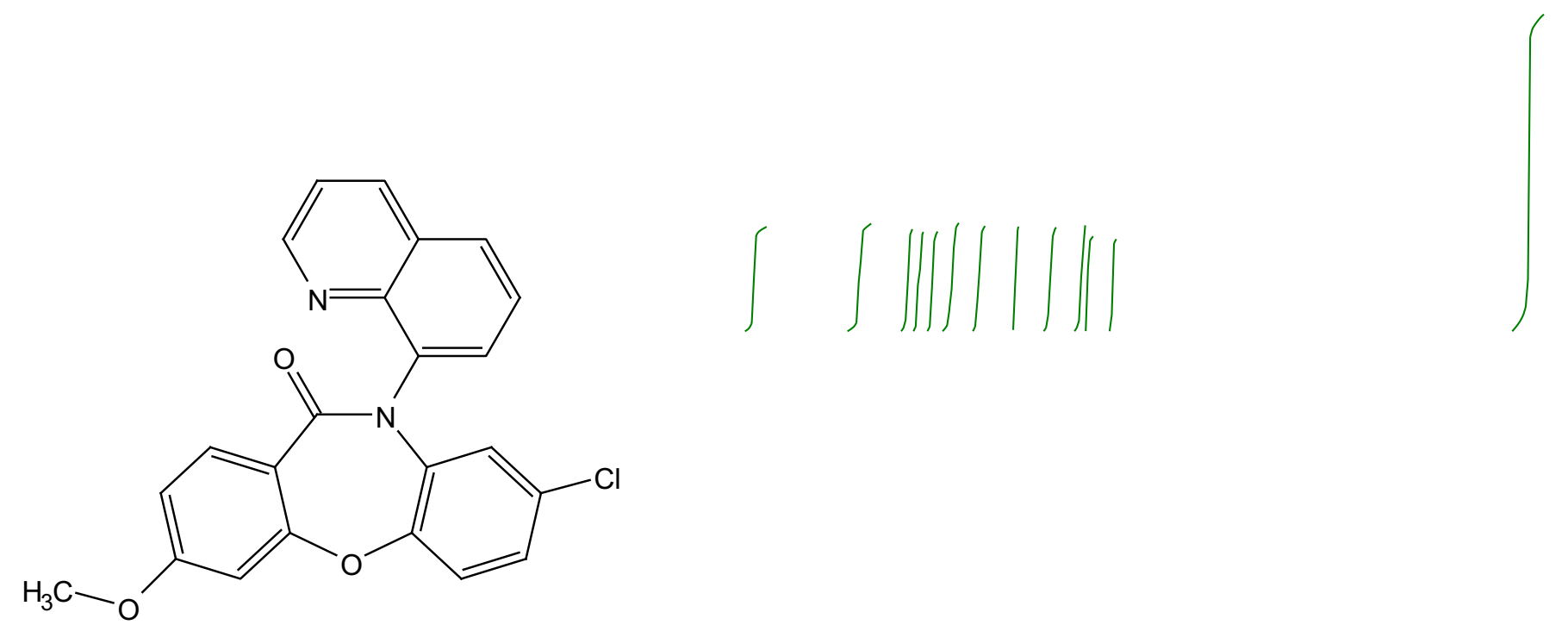

$7 d$

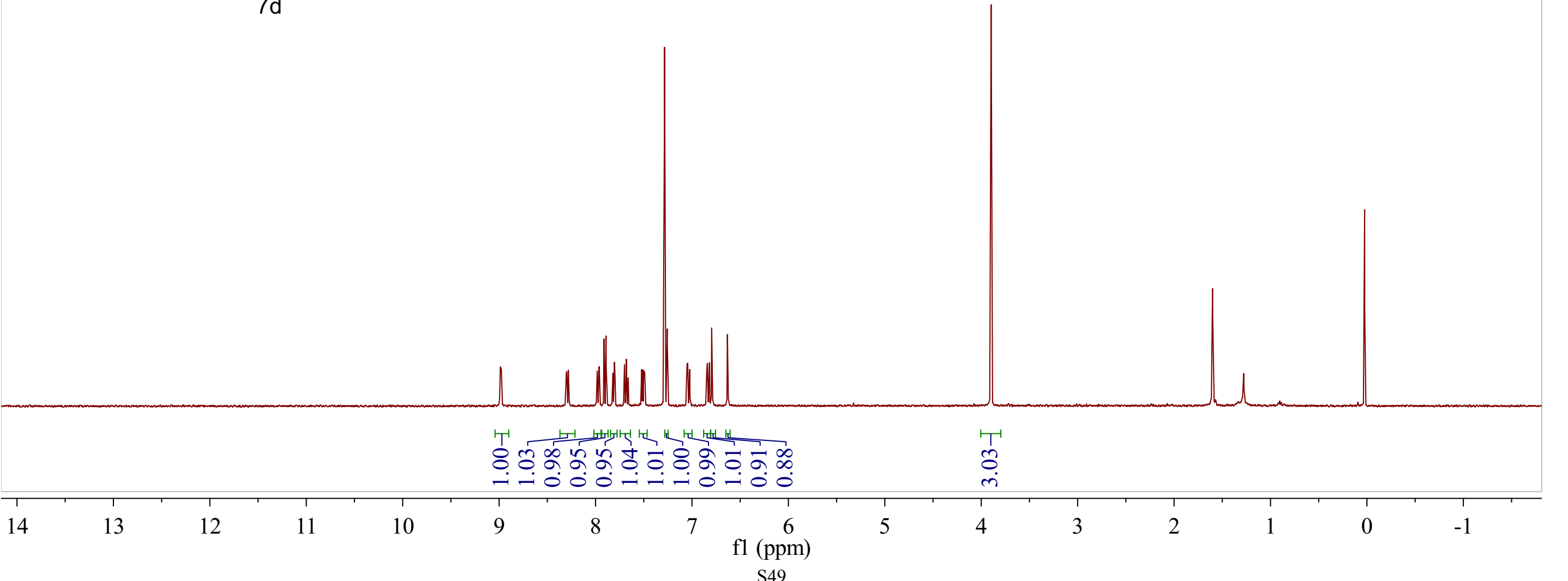




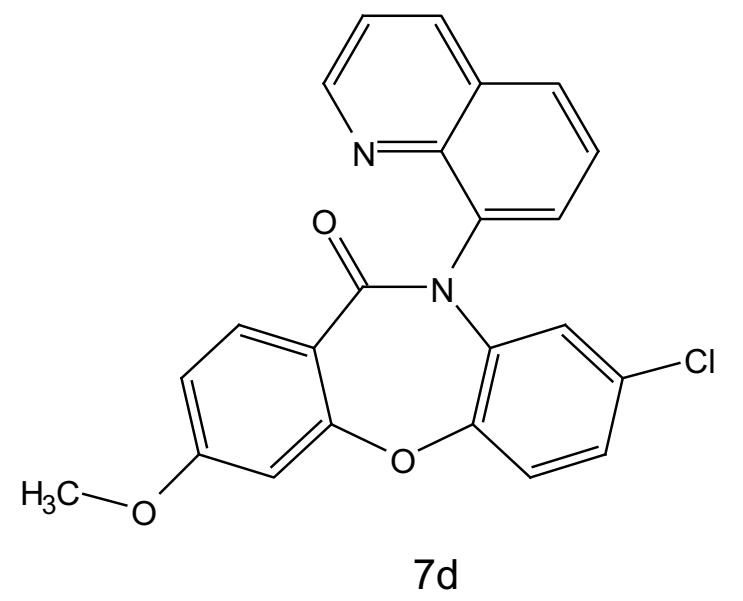

minn

\begin{tabular}{llllllllllllllllllllllllllll}
\hline 230 & 220 & 210 & 200 & 190 & 180 & 170 & 160 & 150 & 140 & 130 & 120 & 110 & 100 & 90 & 80 & 70 & 60 & 50 & 40 & 30 & 20 & 10 & 0 & -10
\end{tabular}




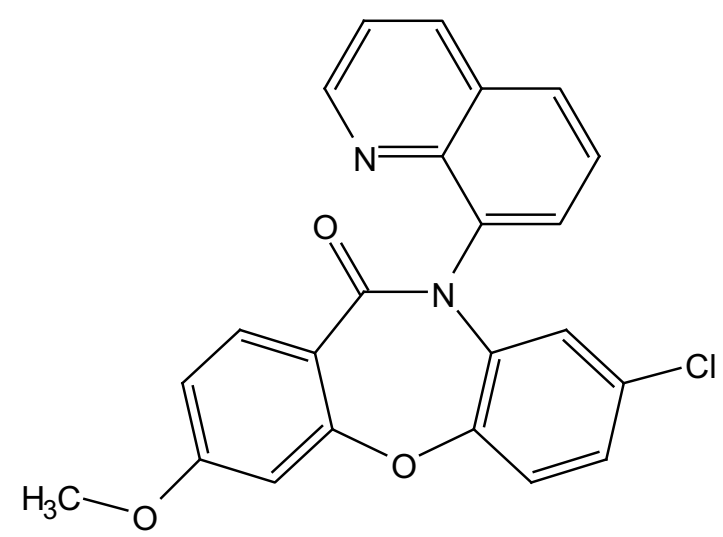

$7 d$

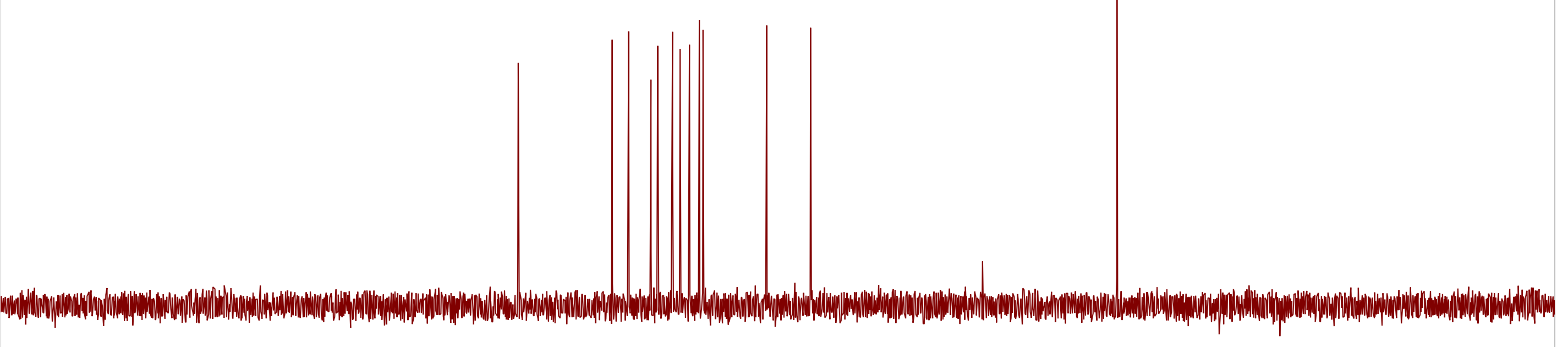

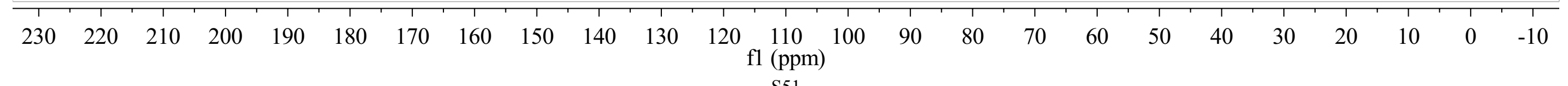



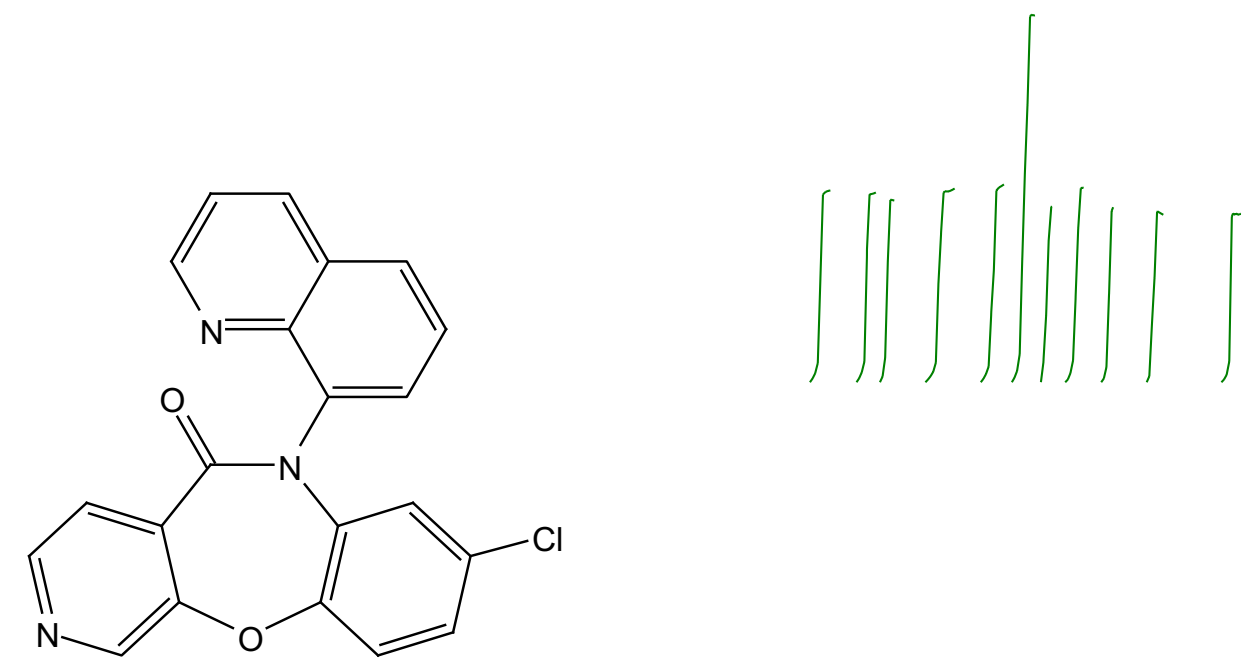

$7 e$

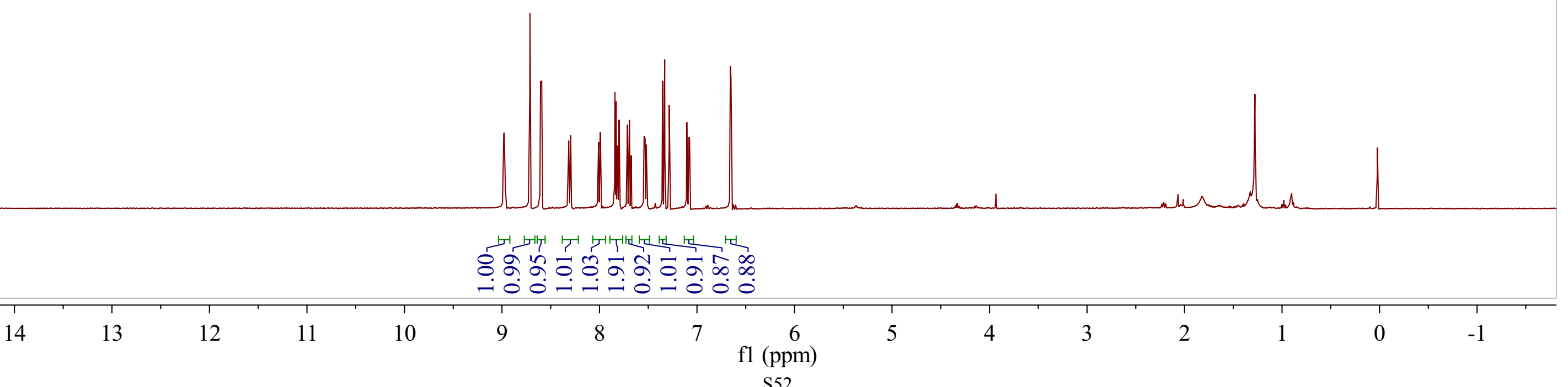




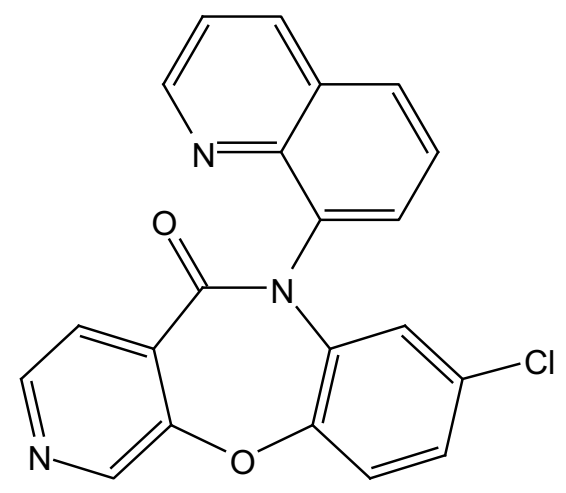

$7 e$

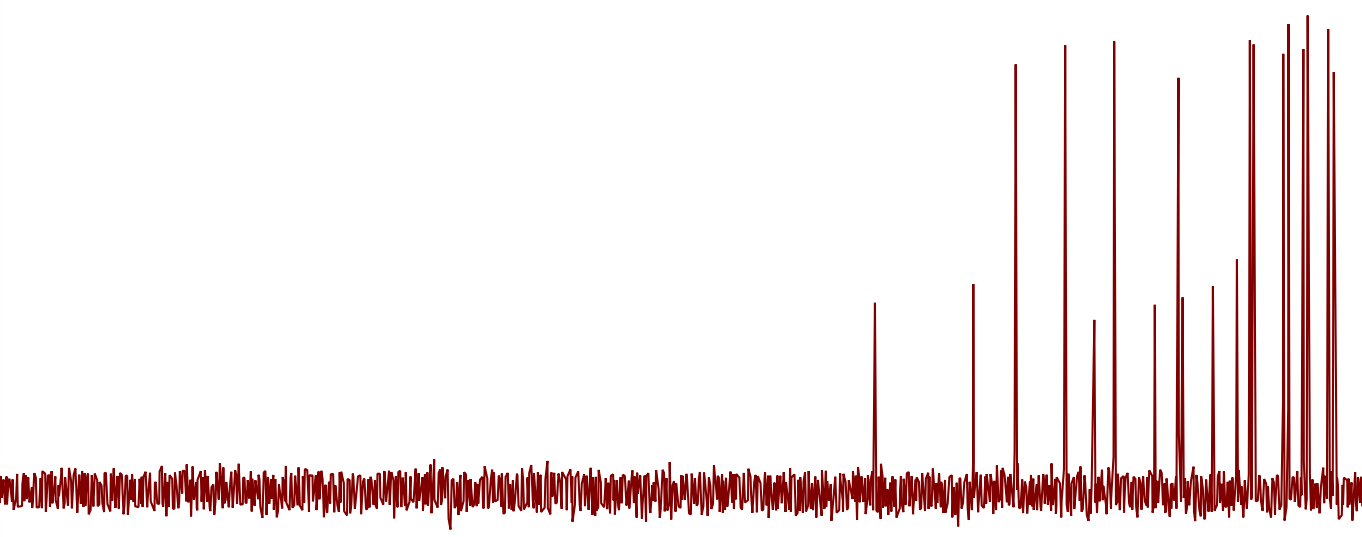

$\begin{array}{lllllllllllllllllllllllllll}240 & 230 & 220 & 210 & 200 & 190 & 180 & 170 & 160 & 150 & 140 & 130 & \begin{array}{c}120 \\ \mathrm{fl}(\mathrm{ppm})\end{array} & 1100 & 90 & 80 & 70 & 60 & 50 & 40 & 30 & 20 & 10 & 0 & -10\end{array}$




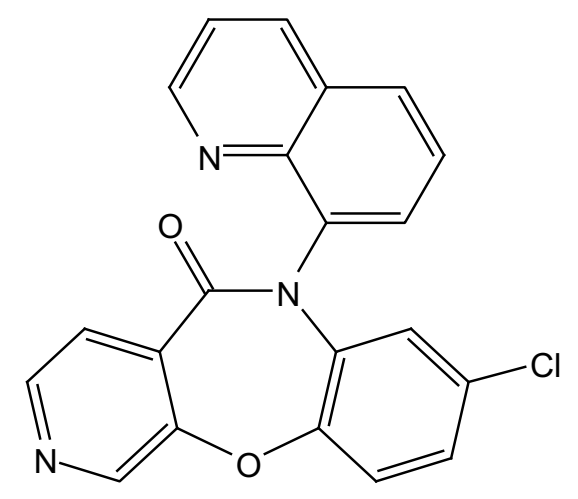

$7 e$

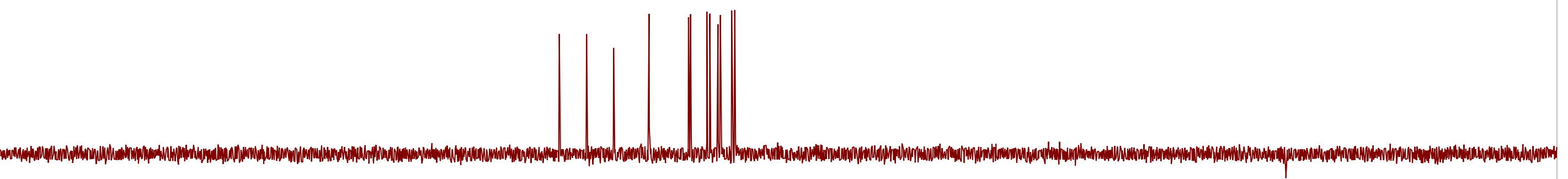

$\begin{array}{rlllllllllllllllllllllllllll}240 & 230 & 220 & 210 & 200 & 190 & 180 & 170 & 160 & 150 & 140 & 130 & \begin{array}{c}120 \\ \mathrm{fl}(\mathrm{ppm})\end{array} & 100 & 90 & 80 & 70 & 60 & 50 & 40 & 30 & 20 & 10 & 0 & -10\end{array}$



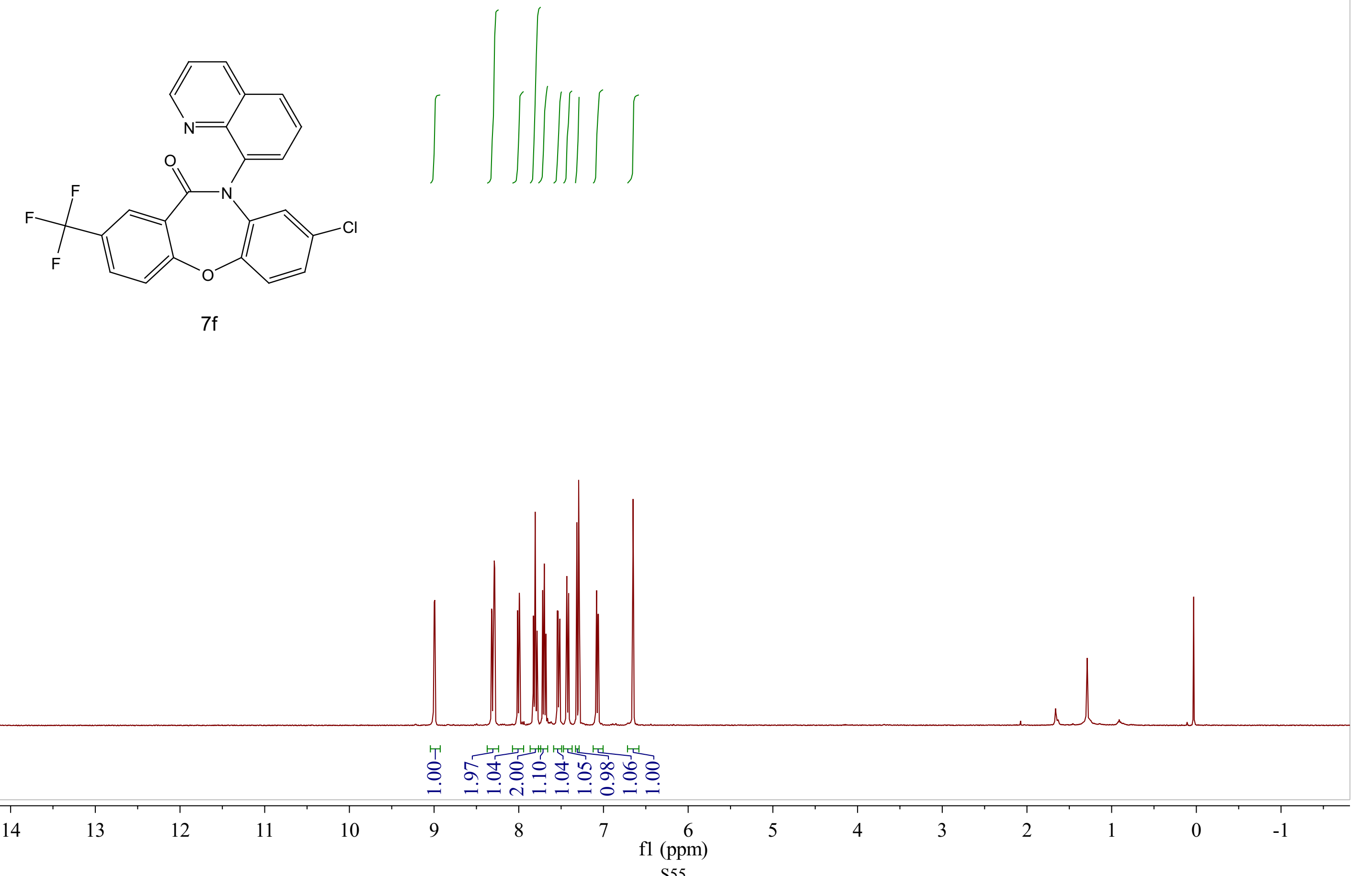


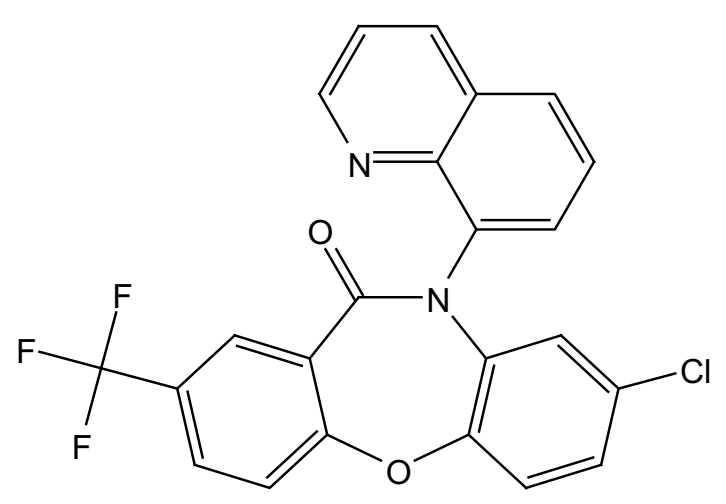

$7 f$
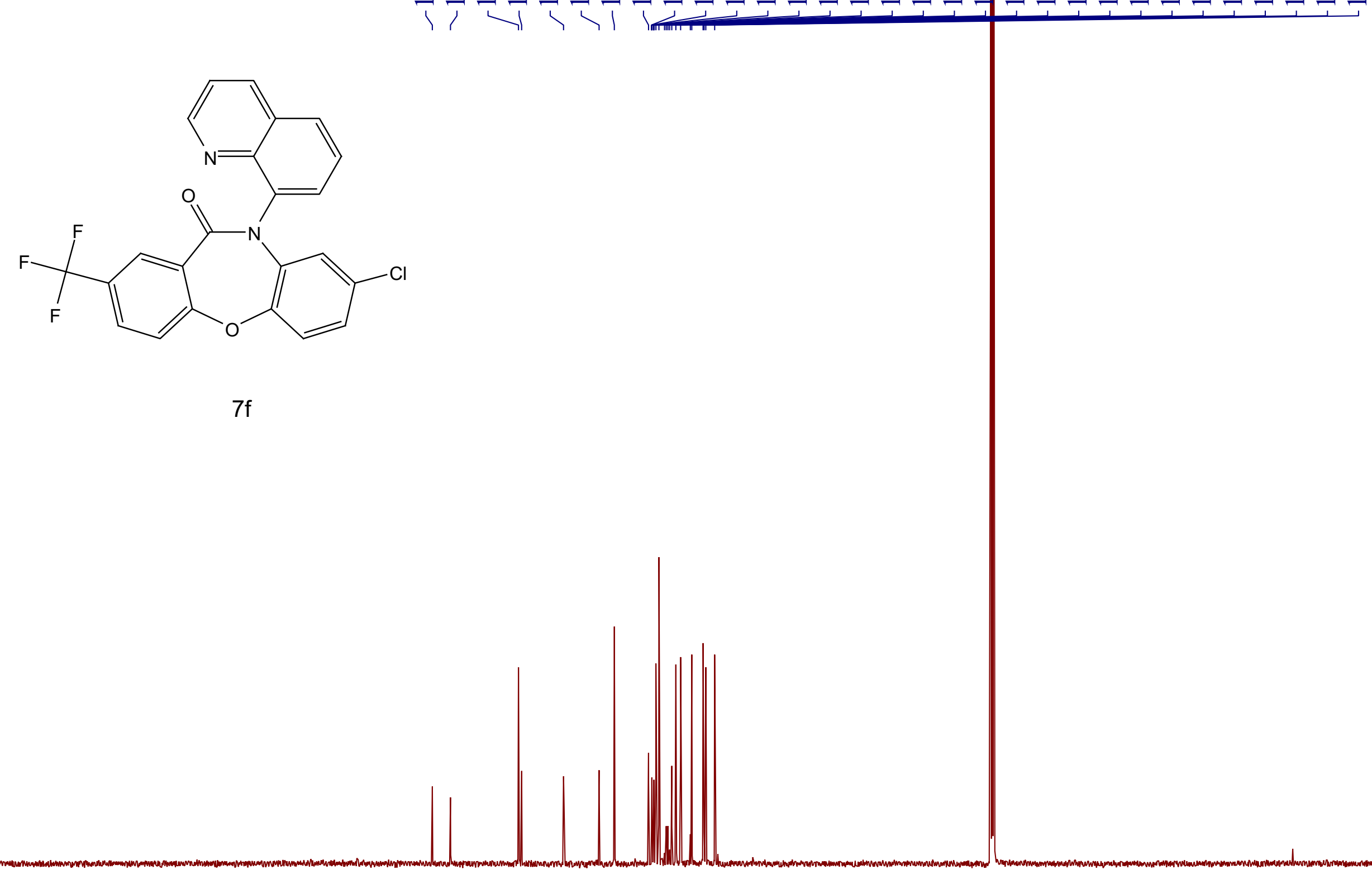

\begin{tabular}{llllllllllllllllllllllllll}
\hline 230 & 220 & 210 & 200 & 190 & 180 & 170 & 160 & 150 & 140 & 130 & 120 & 110 & 100 & 90 & 80 & 70 & 60 & 50 & 40 & 30 & 20 & 10 & 0 & -10
\end{tabular}



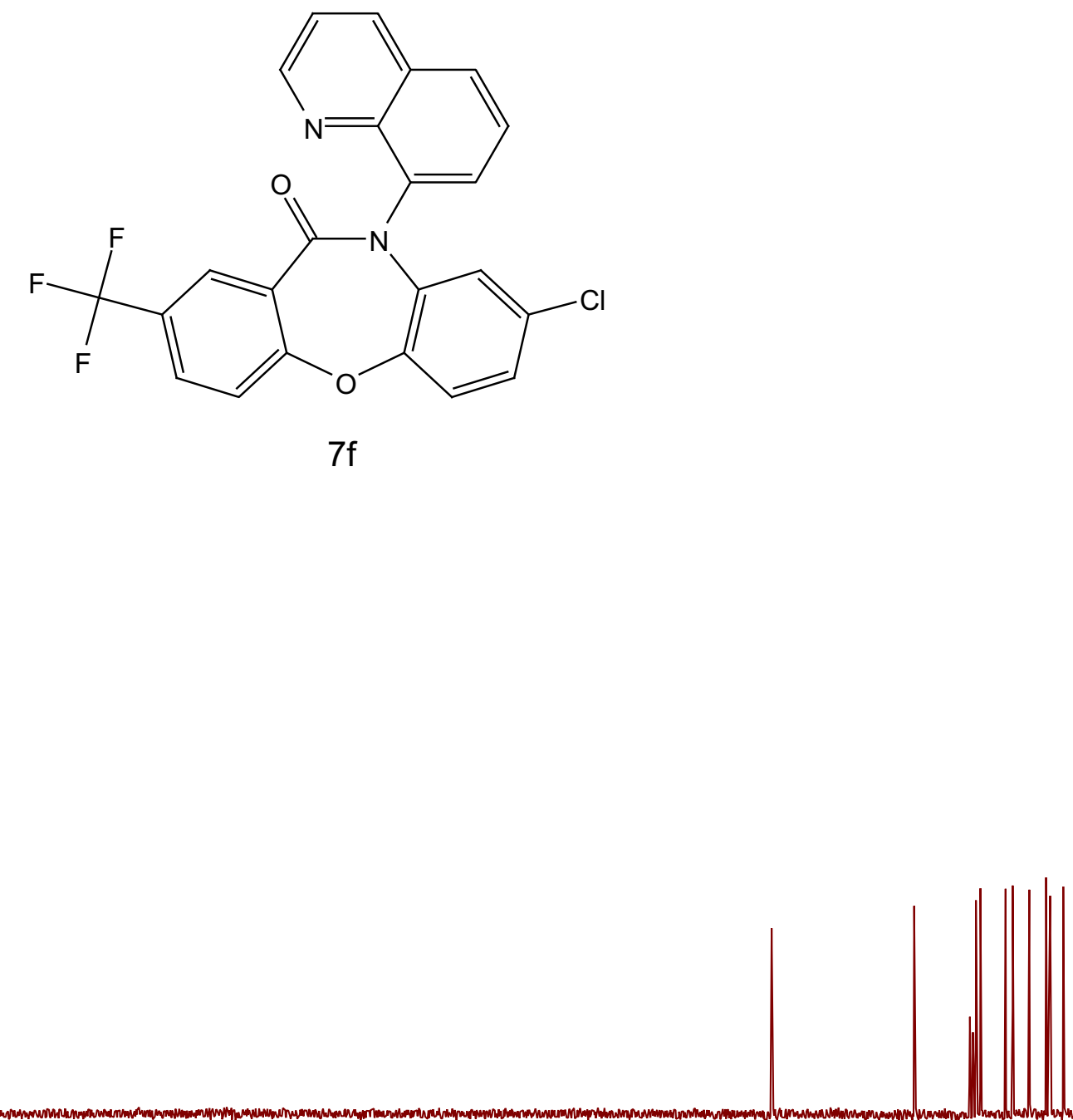

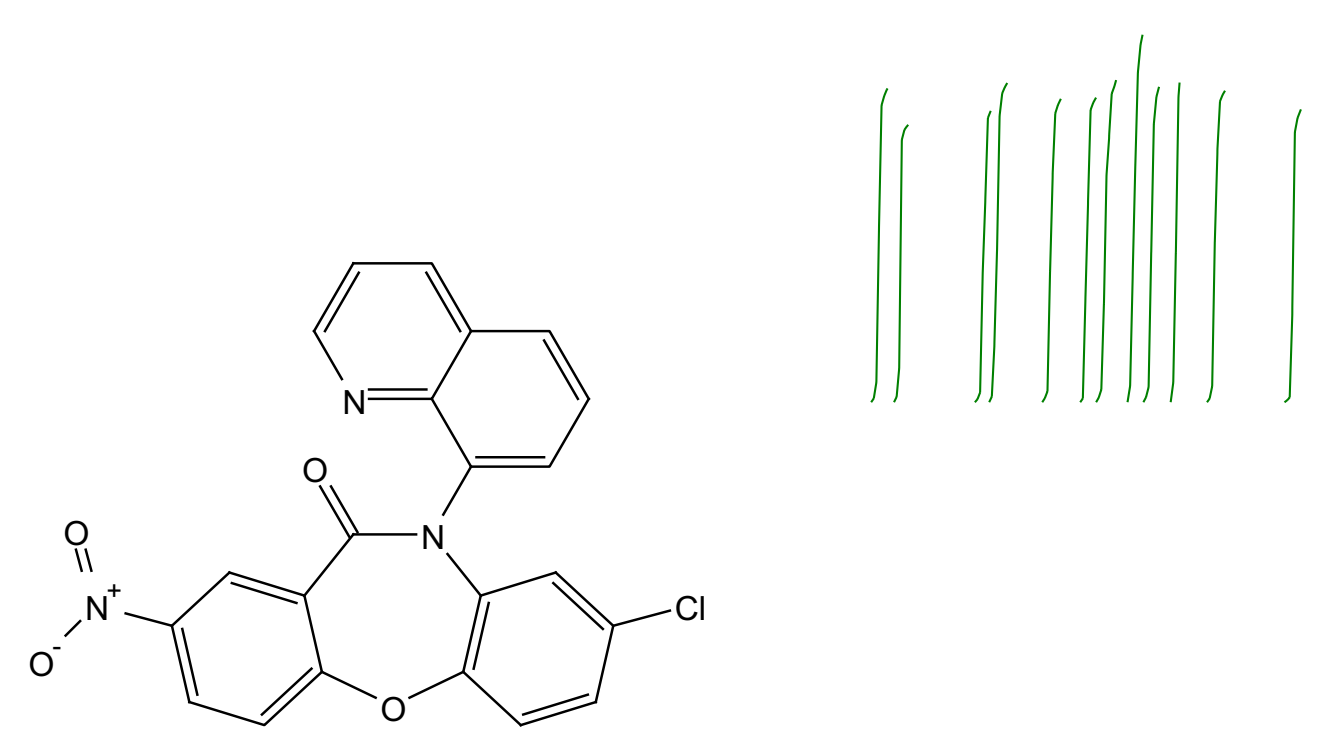

$7 g$

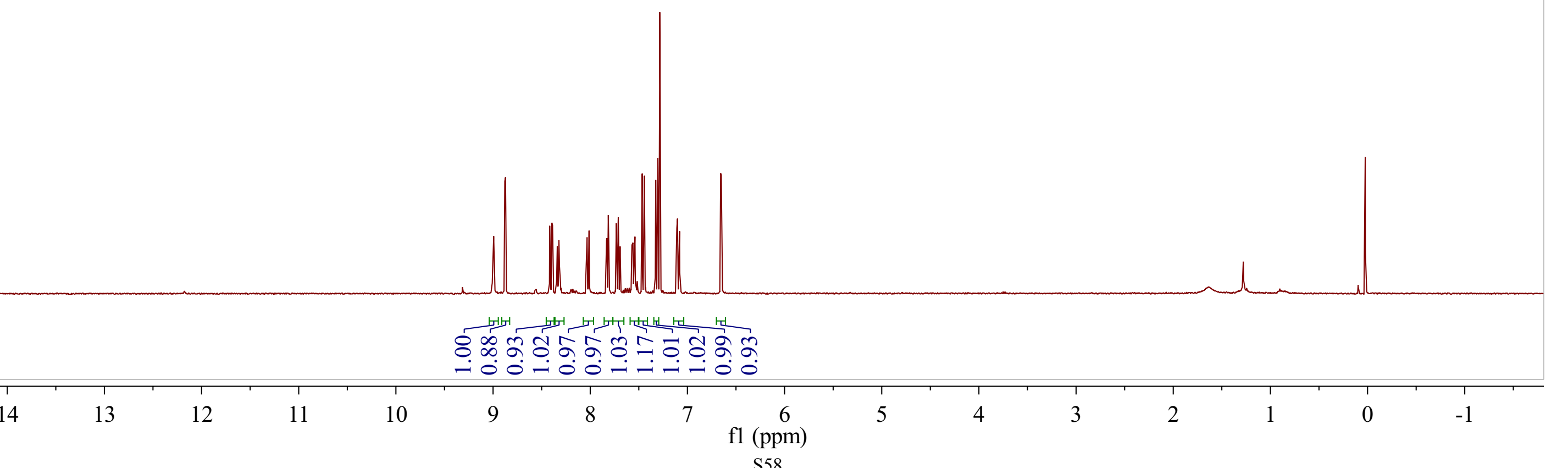




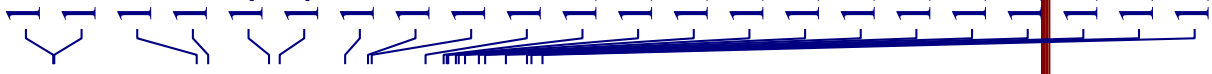

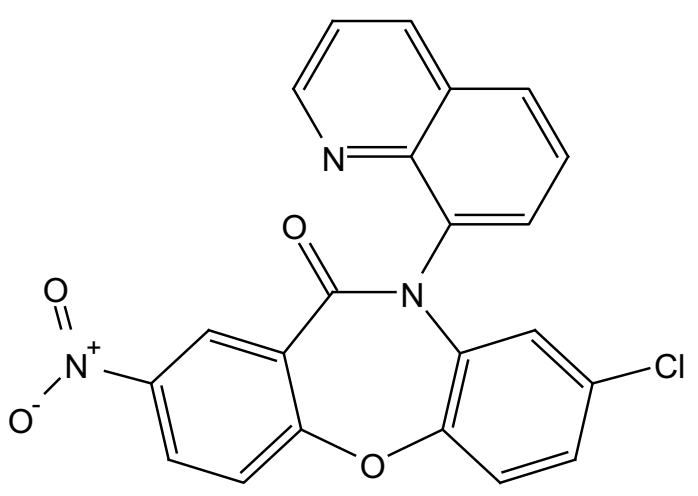

$7 \mathrm{~g}$

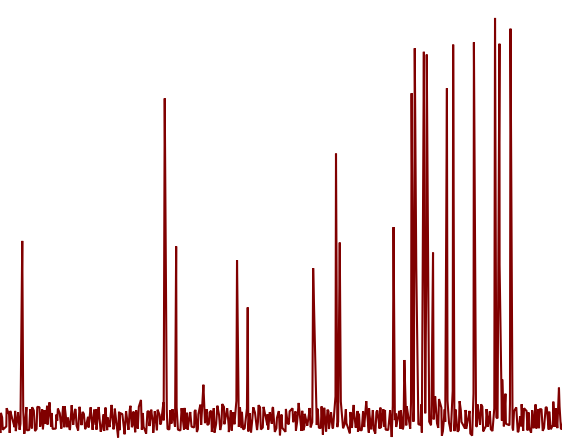

$\begin{array}{rlllllllllllllllllllllllll}230 & 220 & 210 & 200 & 190 & 180 & 170 & 160 & 150 & 140 & 130 & 120 & 110 & 100 & 90 & 80 & 70 & 60 & 50 & 40 & 30 & 20 & 10 & 0 & -10\end{array}$




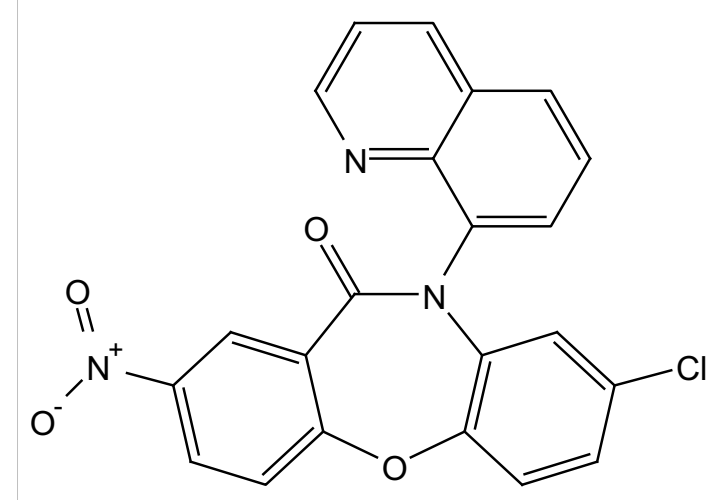

$7 \mathrm{~g}$

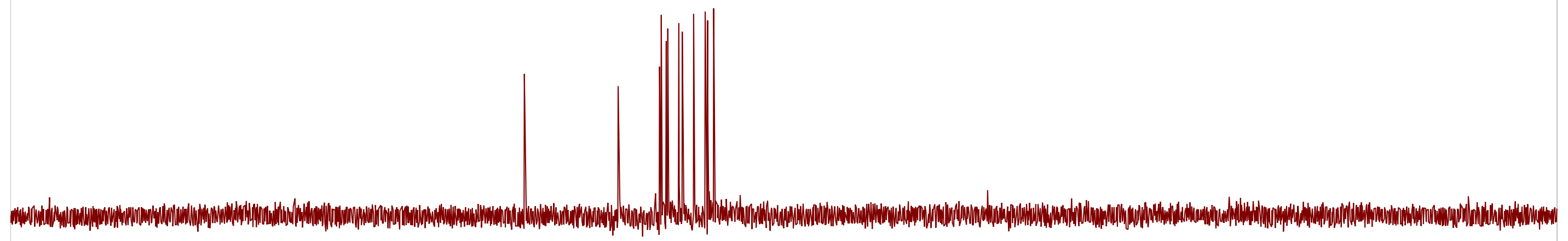

$\begin{array}{rllllllllllllllllllllllll}230 & 220 & 210 & 200 & 190 & 180 & 170 & 160 & 150 & 140 & 130 & 120 & 110 & 100 & 90 & 80 & 70 & 60 & 50 & 40 & 30 & 20 & 10 & 0 & -10\end{array}$



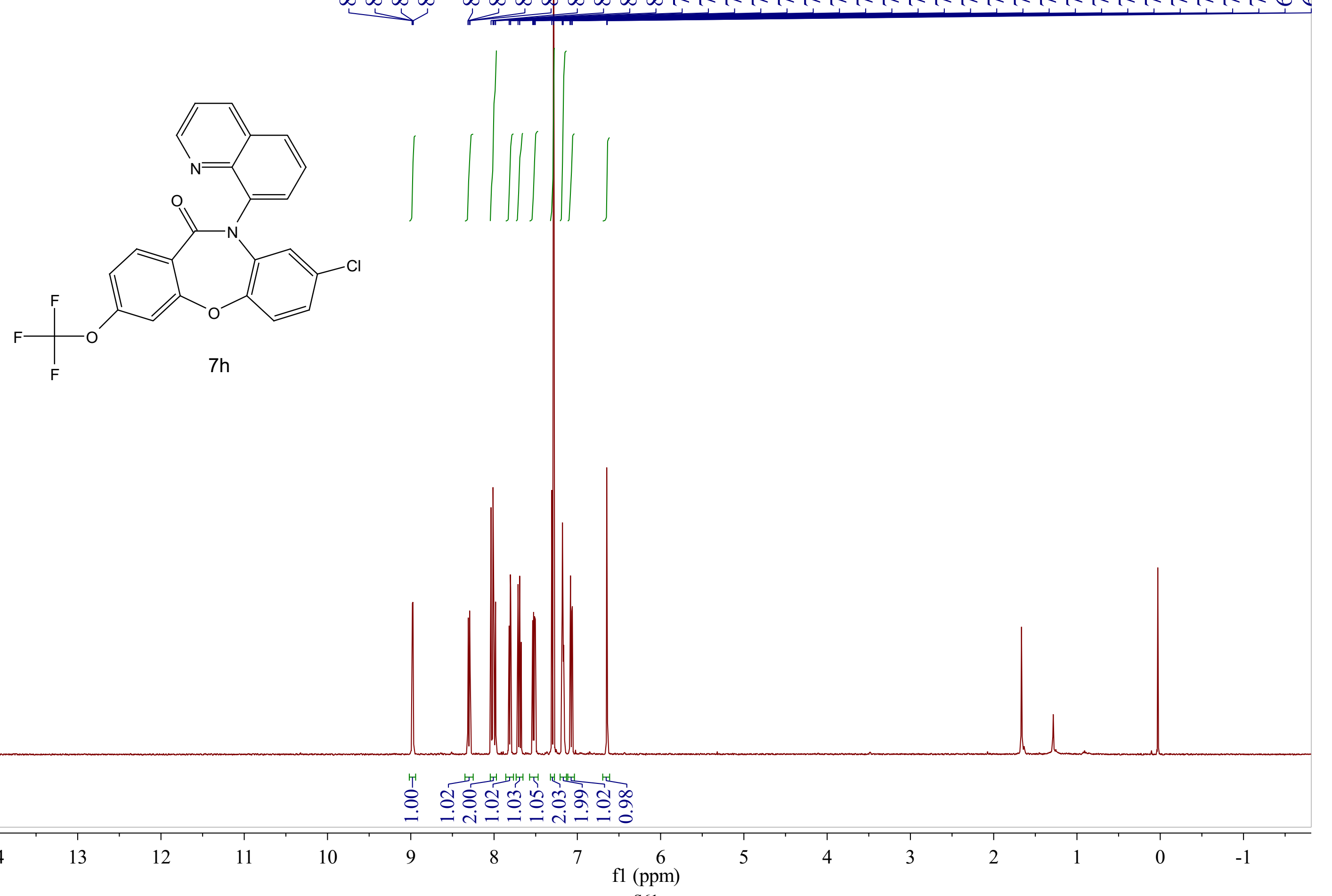


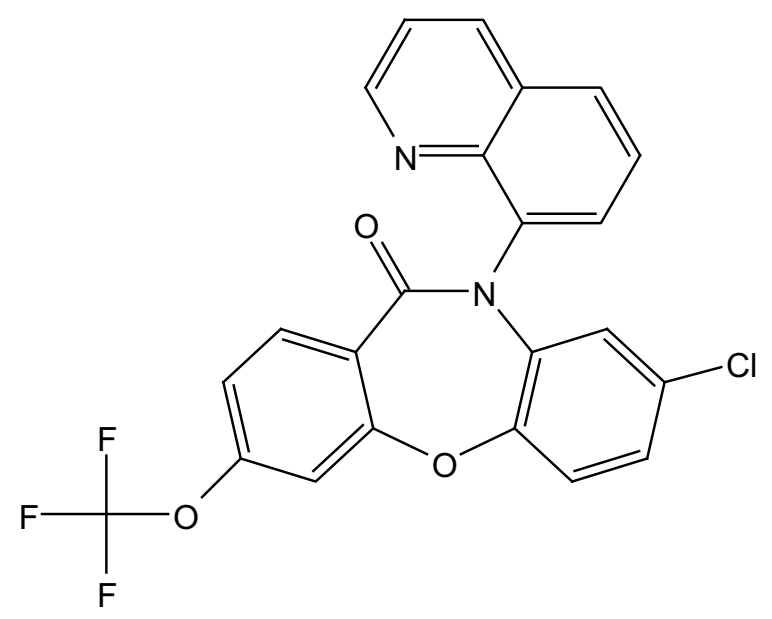

$7 \mathrm{~h}$

$\begin{array}{rllllllllllllllllllllllllll}240 & 230 & 220 & 210 & 200 & 190 & 180 & 170 & 160 & 150 & 140 & 130 & 120 & 110 & 100 & 90 & 80 & 70 & 60 & 50 & 40 & 30 & 20 & 10 & 0 & -10\end{array}$



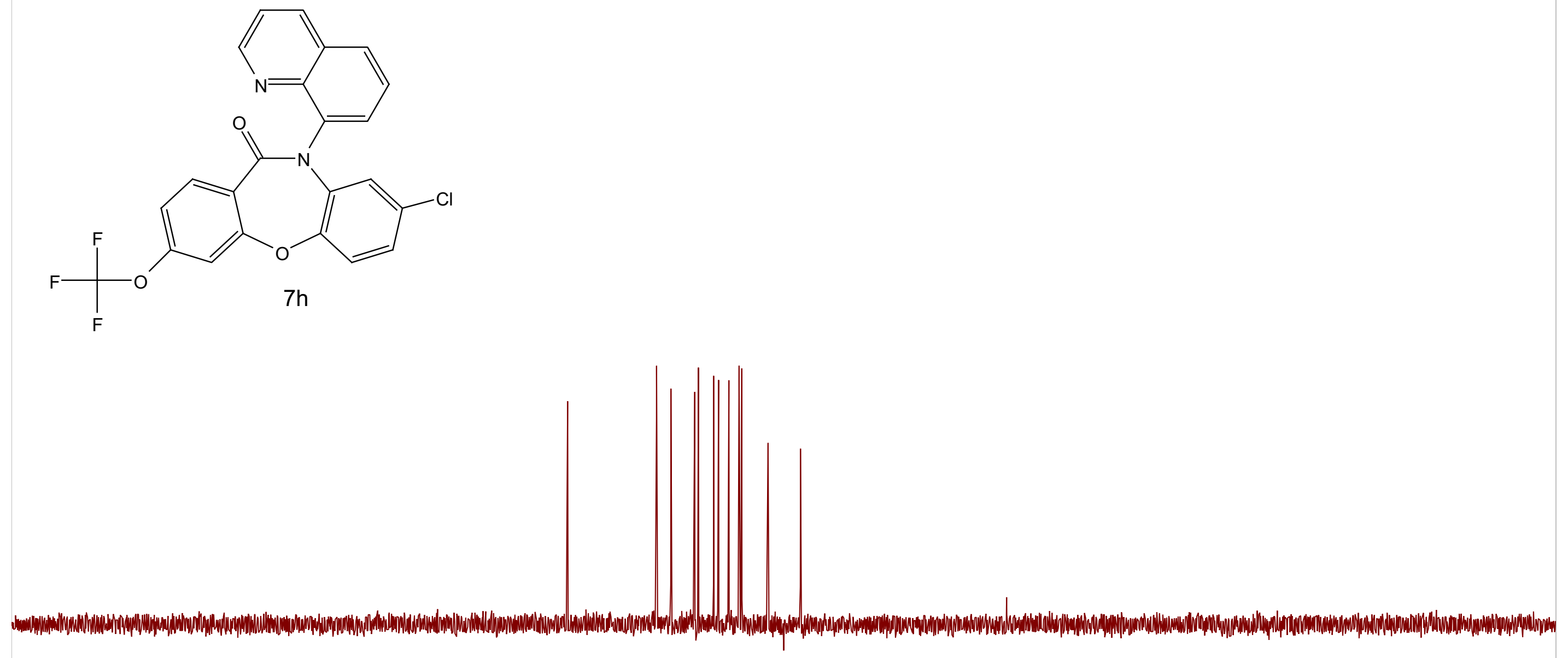

$\begin{array}{rllllllllllllllllllllllllll}240 & 230 & 220 & 210 & 200 & 190 & 180 & 170 & 160 & 150 & 140 & 130 & 120 & 110 & 100 & 90 & 80 & 70 & 60 & 50 & 40 & 30 & 20 & 10 & 0 & -10\end{array}$



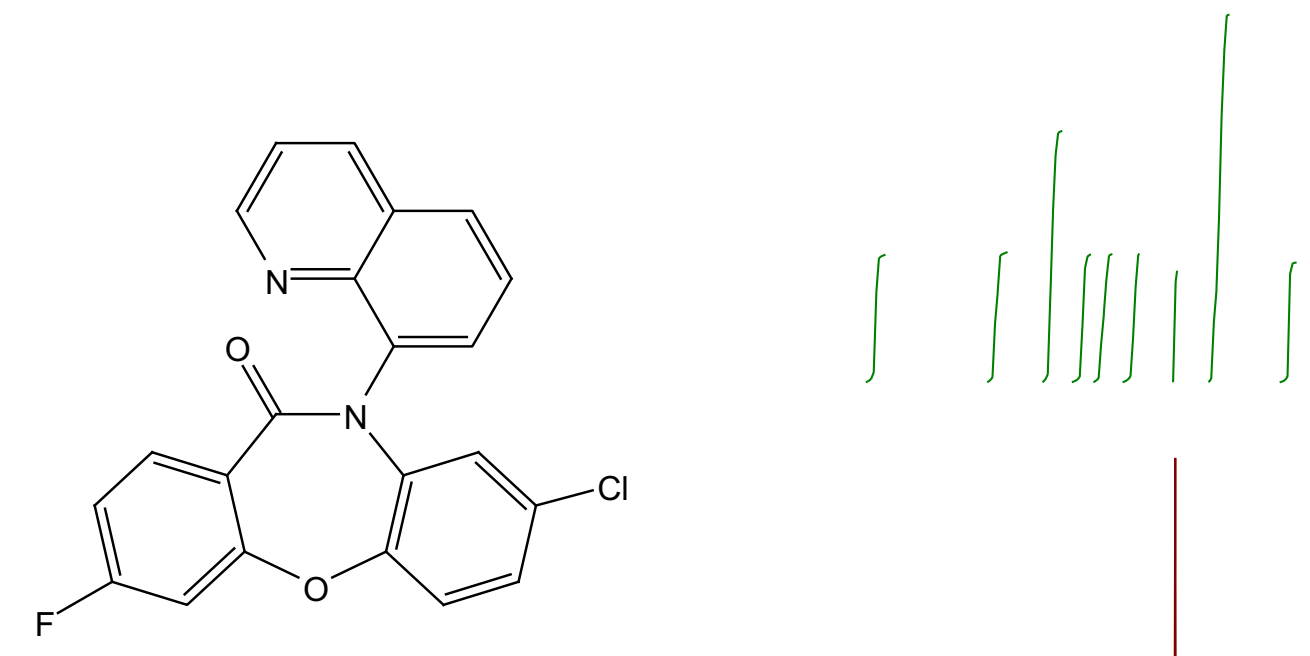

$7 i$

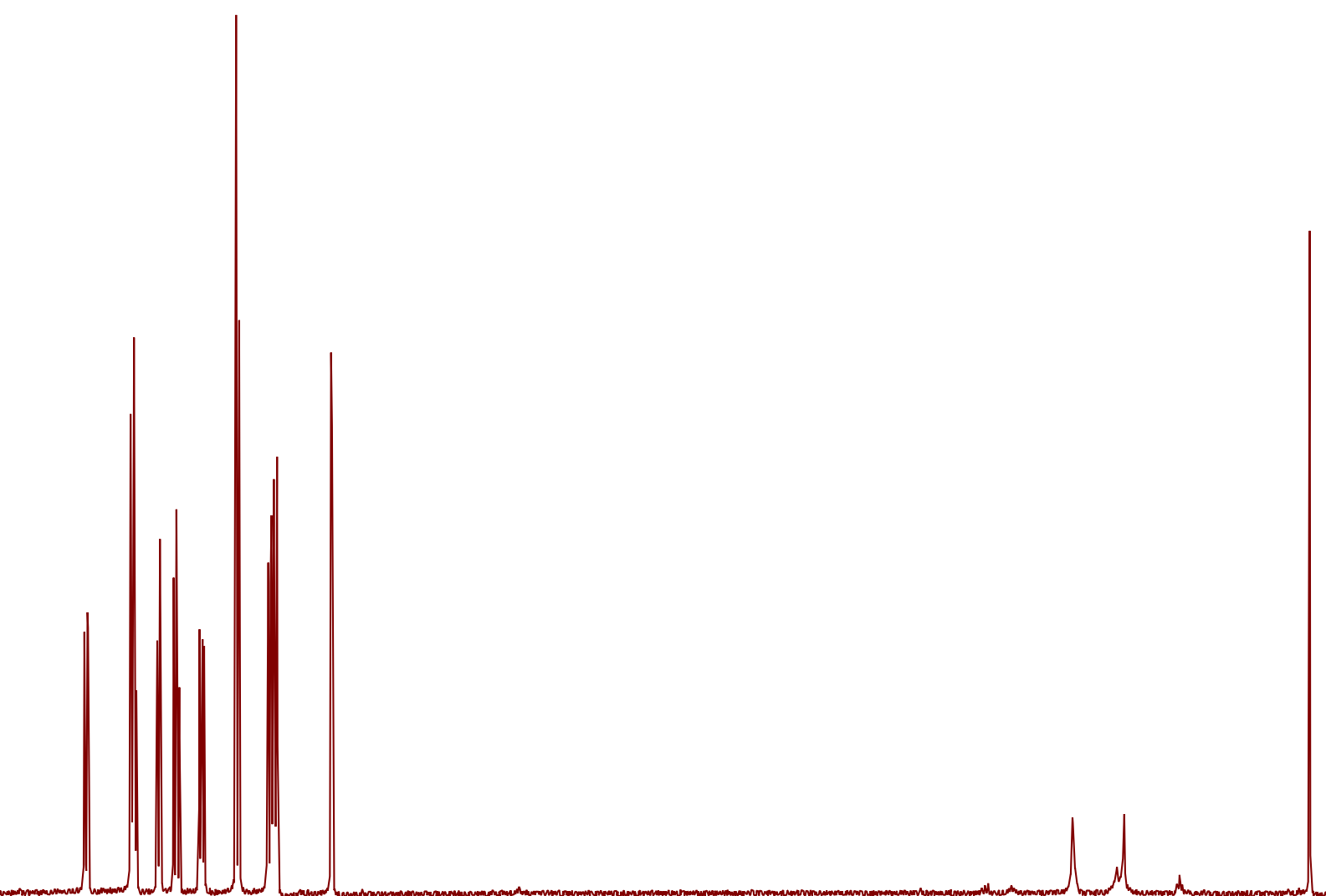

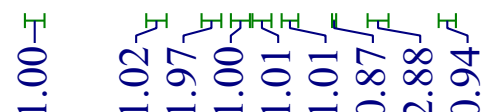

12

11

10

9

8

7

$$
\text { fl (ppm) }
$$




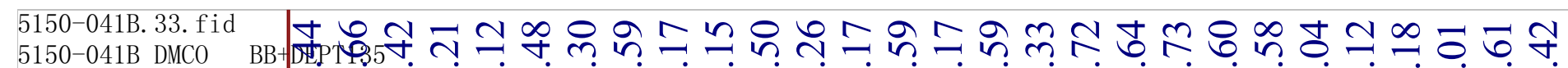

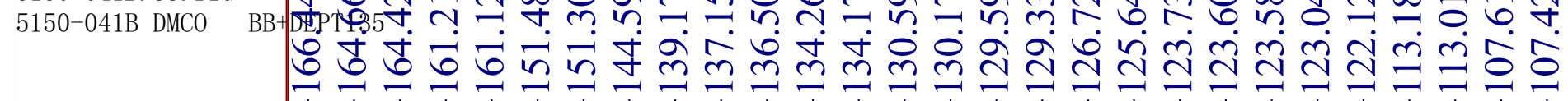

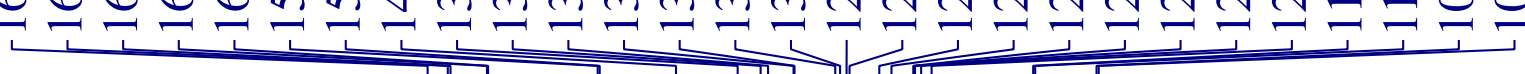

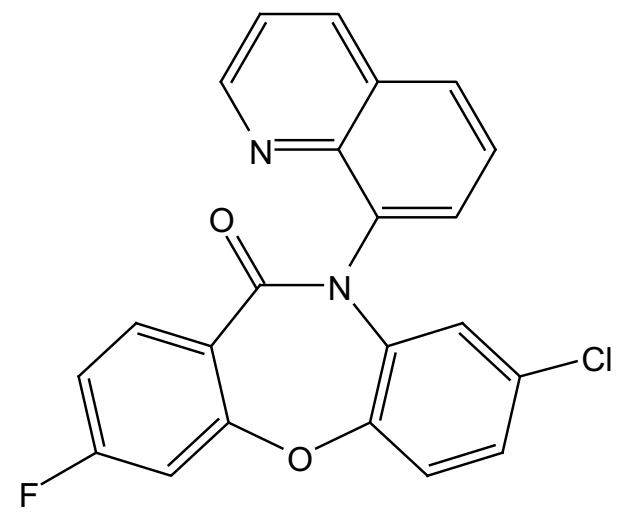

$7 \mathrm{i}$

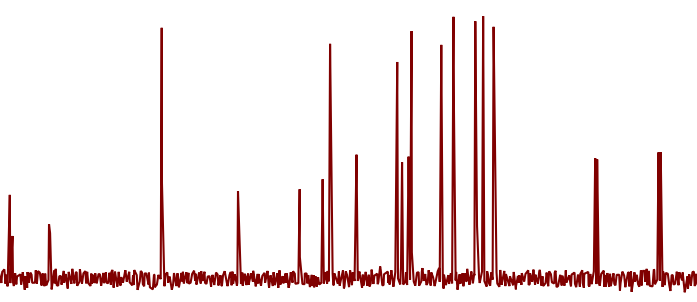

\begin{tabular}{lllllllllllllllllllllllll}
\hline 230 & 220 & 210 & 200 & 190 & 180 & 170 & 160 & 150 & 140 & 130 & 120 & 110 & 100 & 90 & 80 & 70 & 60 & 50 & 40 & 30 & 20 & 10 & 0 & -10
\end{tabular}




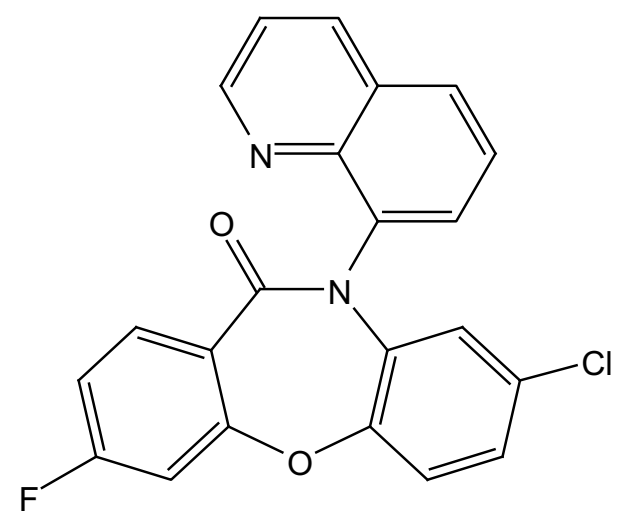

$7 i$

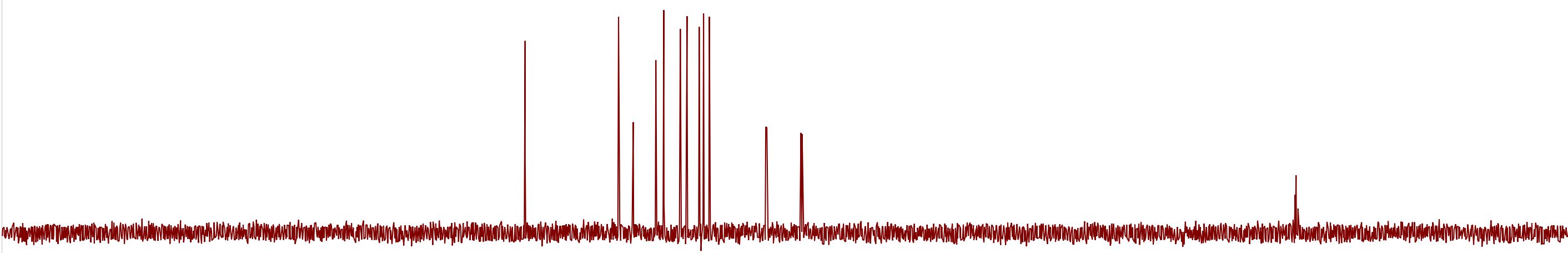

\begin{tabular}{lllllllllllllllllllllllll}
\hline 230 & 220 & 210 & 200 & 190 & 180 & 170 & 160 & 150 & 140 & 130 & 120 & $\begin{array}{l}110 \\
\mathrm{fl}(\mathrm{ppm})\end{array}$ & 100 & 90 & 80 & 70 & 60 & 50 & 40 & 30 & 20 & 10 & 0 & -10
\end{tabular}



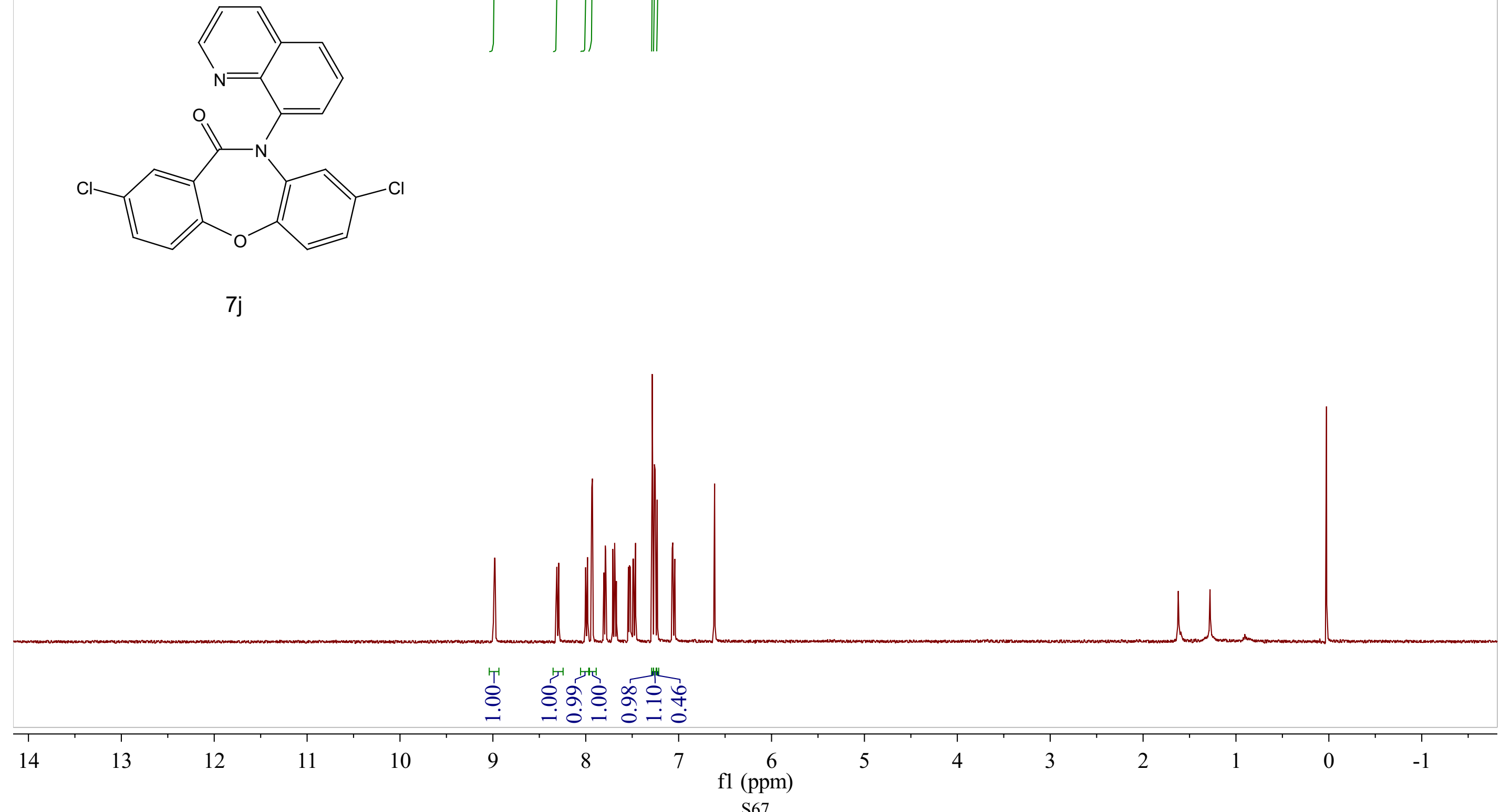

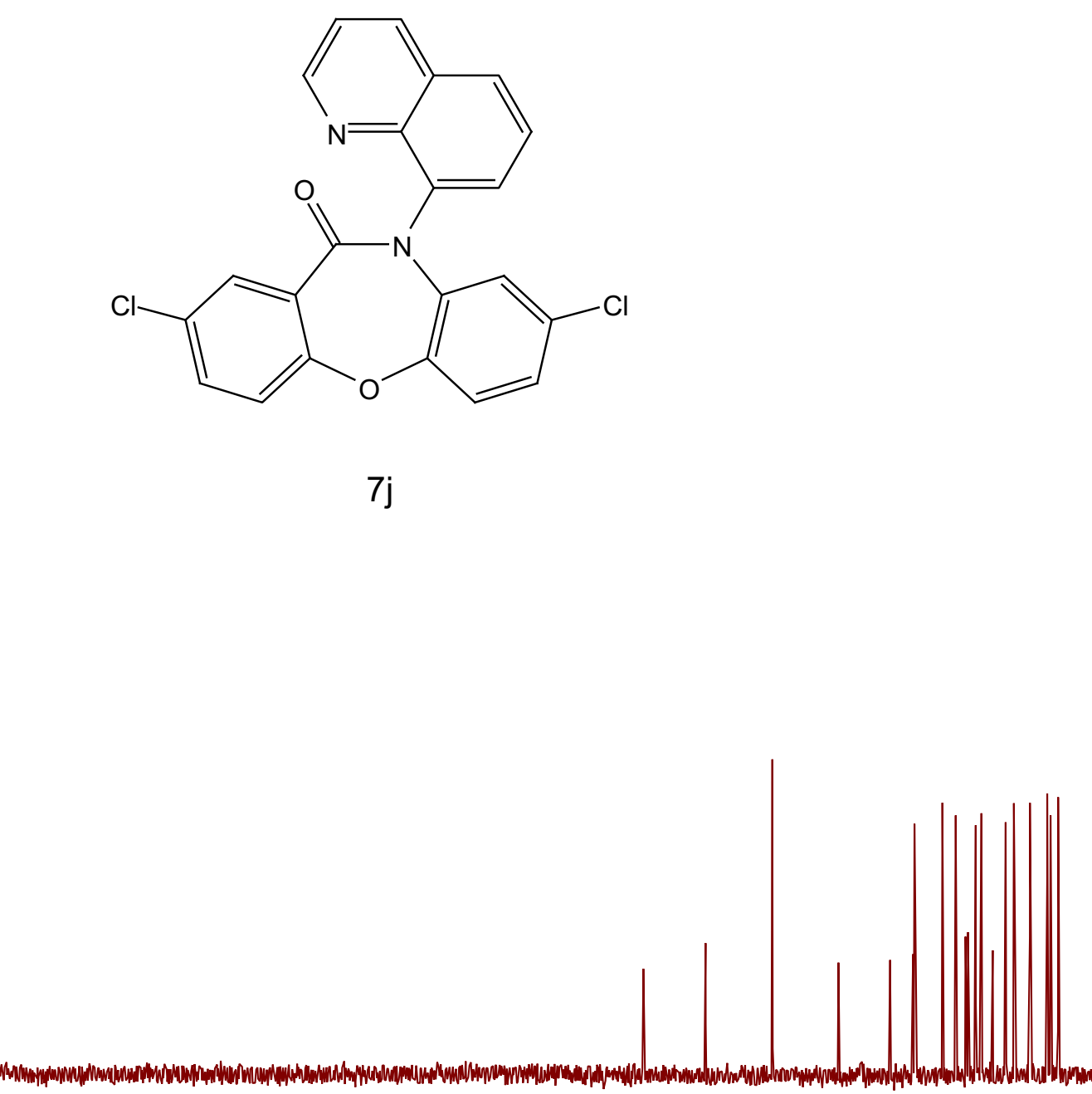

\begin{tabular}{lllllllllllllllllllllllll}
\hline 230 & 220 & 210 & 200 & 190 & 180 & 170 & 160 & 150 & 140 & 130 & 120 & 110 & 100 & 90 & 80 & 70 & 60 & 50 & 40 & 30 & 20 & 10 & 0 & -10
\end{tabular}




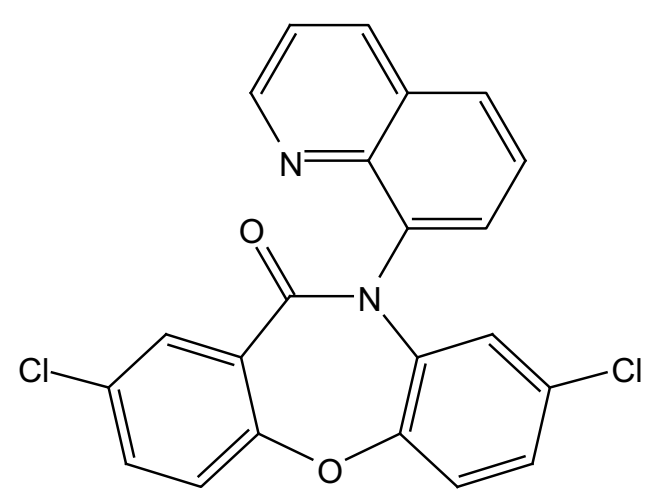

7j

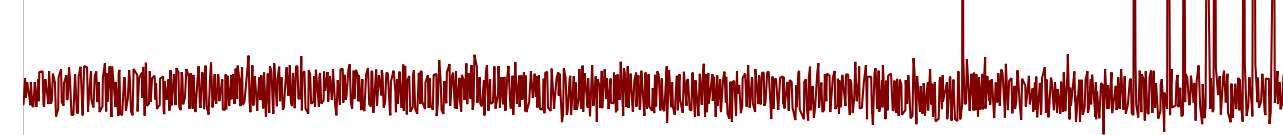

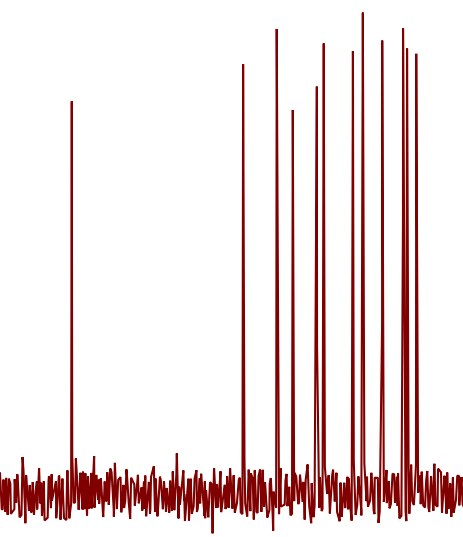

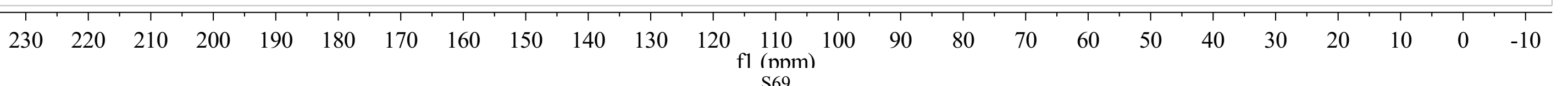



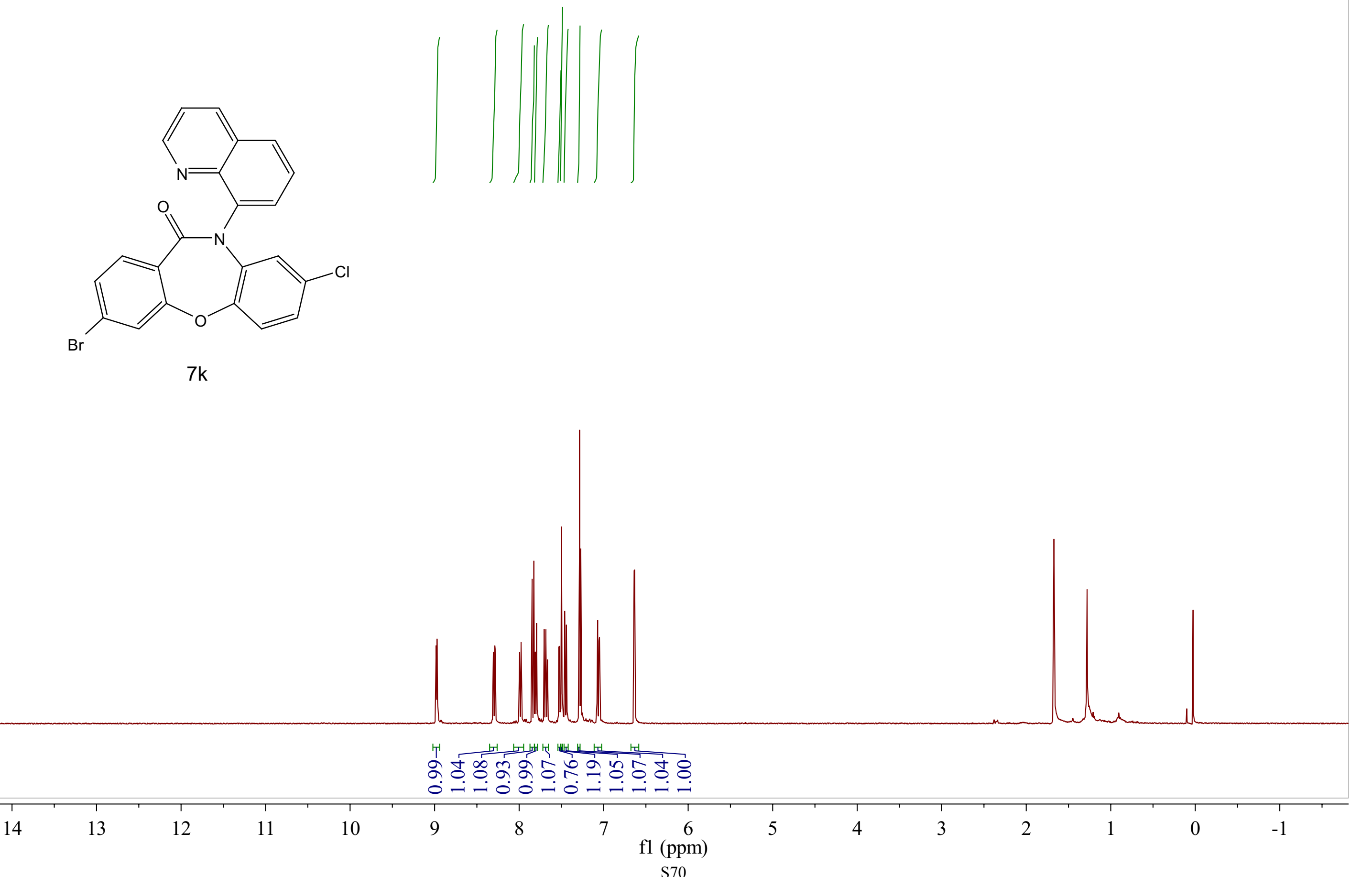


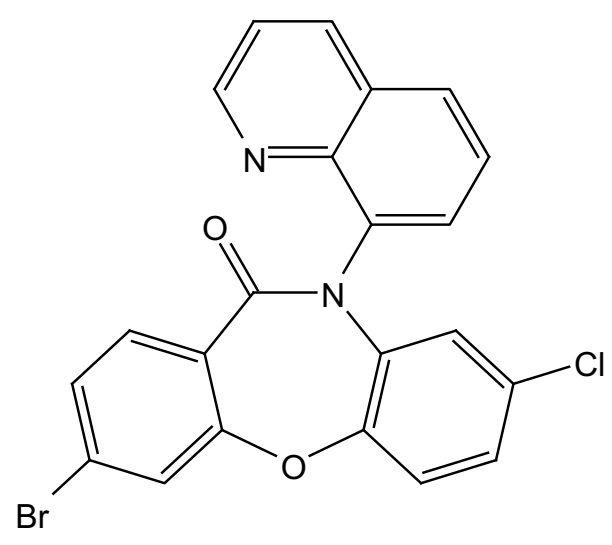

$7 \mathrm{k}$

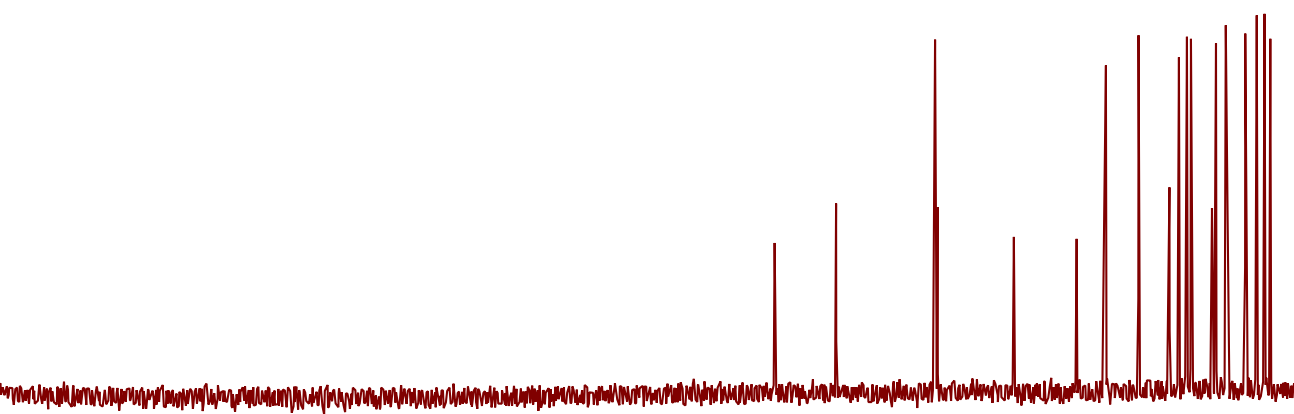

$\begin{array}{lllllllllllllllllllllllll}230 & 220 & 210 & 200 & 190 & 180 & 170 & 160 & 150 & 140 & 130 & 120 & \begin{array}{l}110 \\ \text { fl (ppm) }\end{array} & 100 & 90 & 80 & 70 & 60 & 50 & 40 & 30 & 20 & 10 & 0 & -10\end{array}$




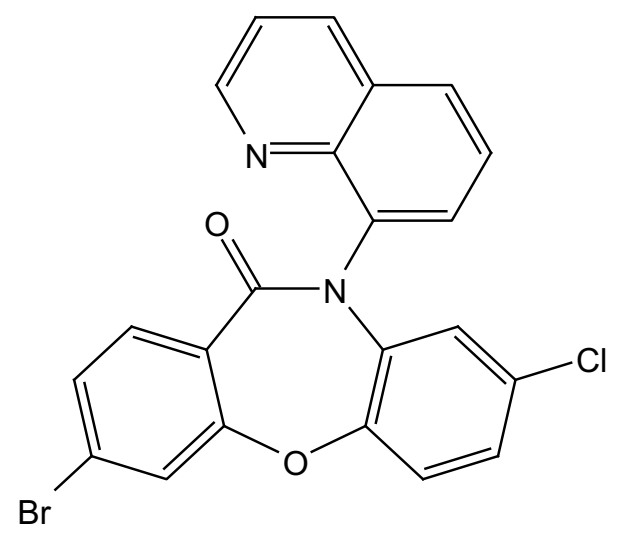

$7 \mathrm{k}$

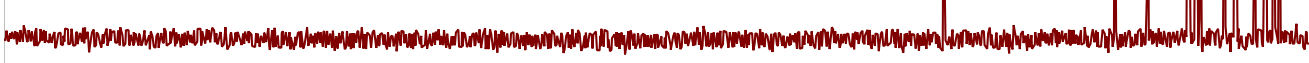

\begin{tabular}{lllllllllllllllllllllllll}
\hline 230 & 220 & 210 & 200 & 190 & 180 & 170 & 160 & 150 & 140 & 130 & 120 & 110 & 100 & 90 & 80 & 70 & 60 & 50 & 40 & 30 & 20 & 10 & 0 & -10
\end{tabular}



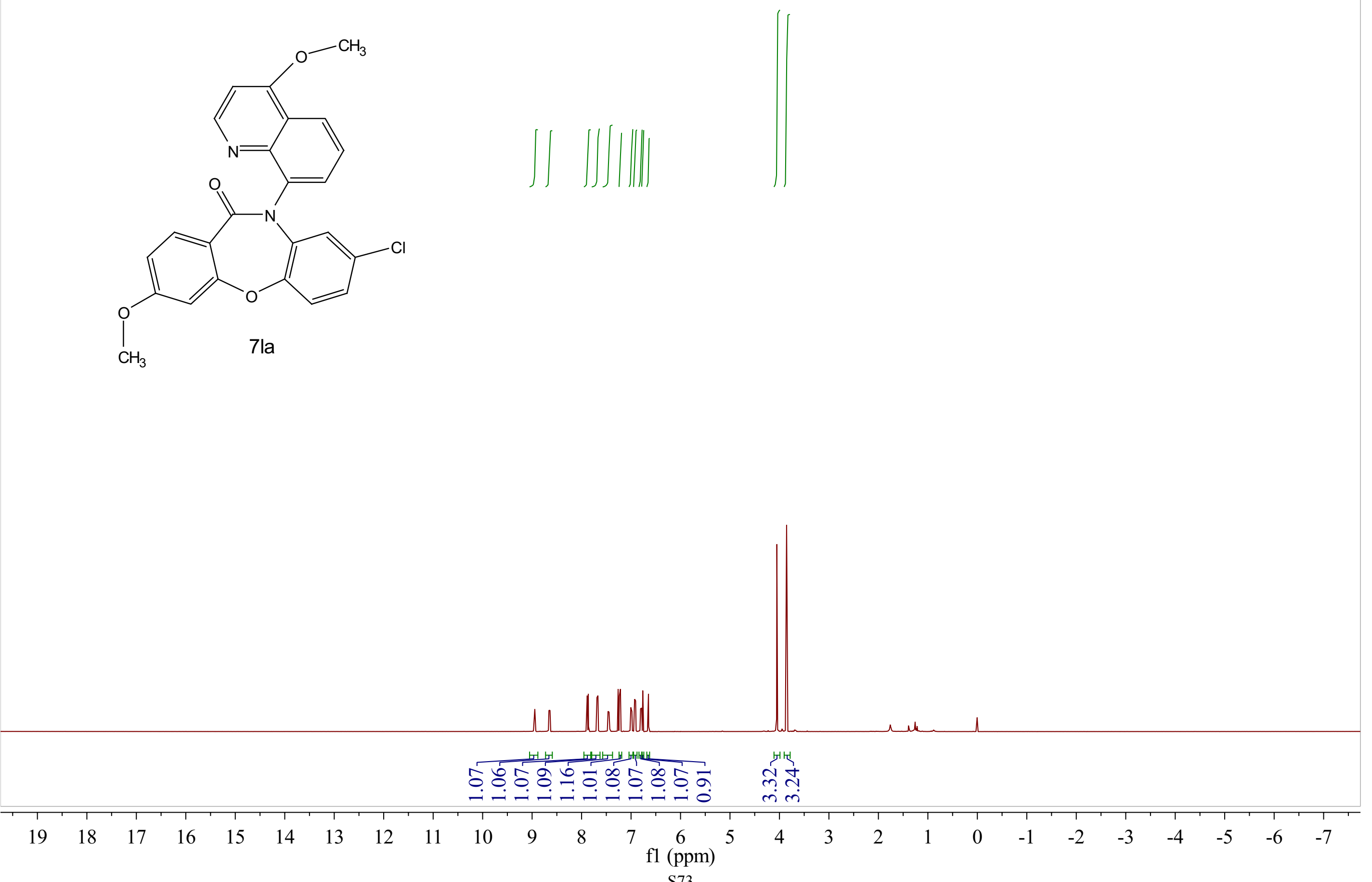


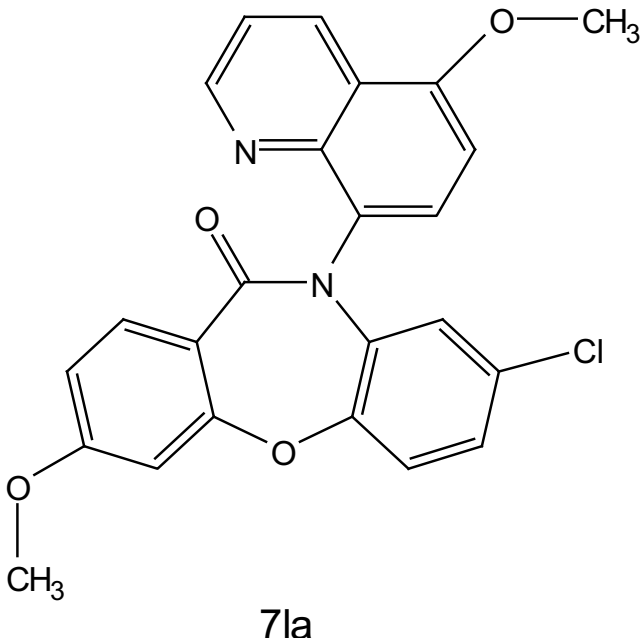

7 la

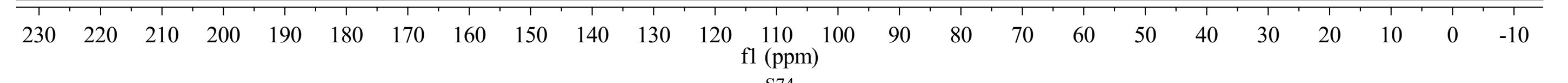




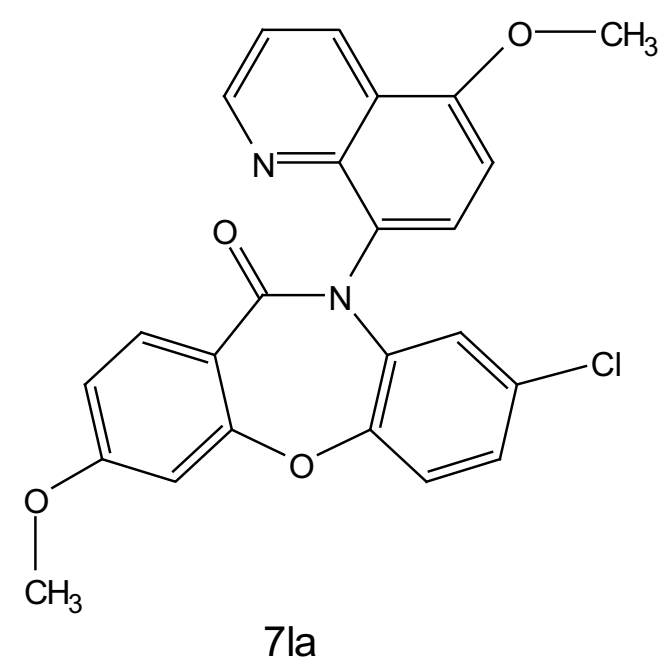

7 la

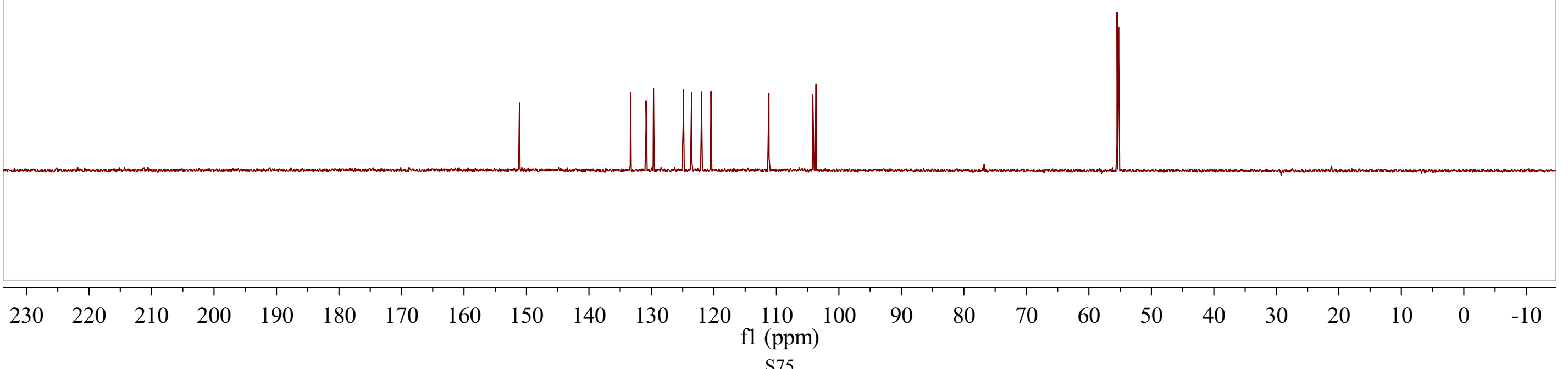




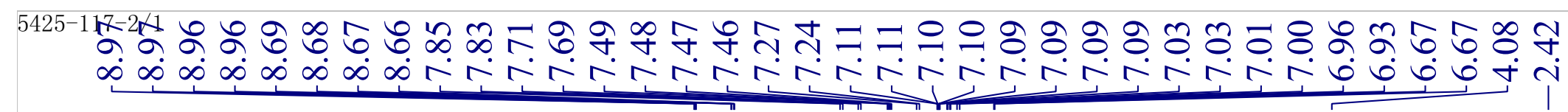
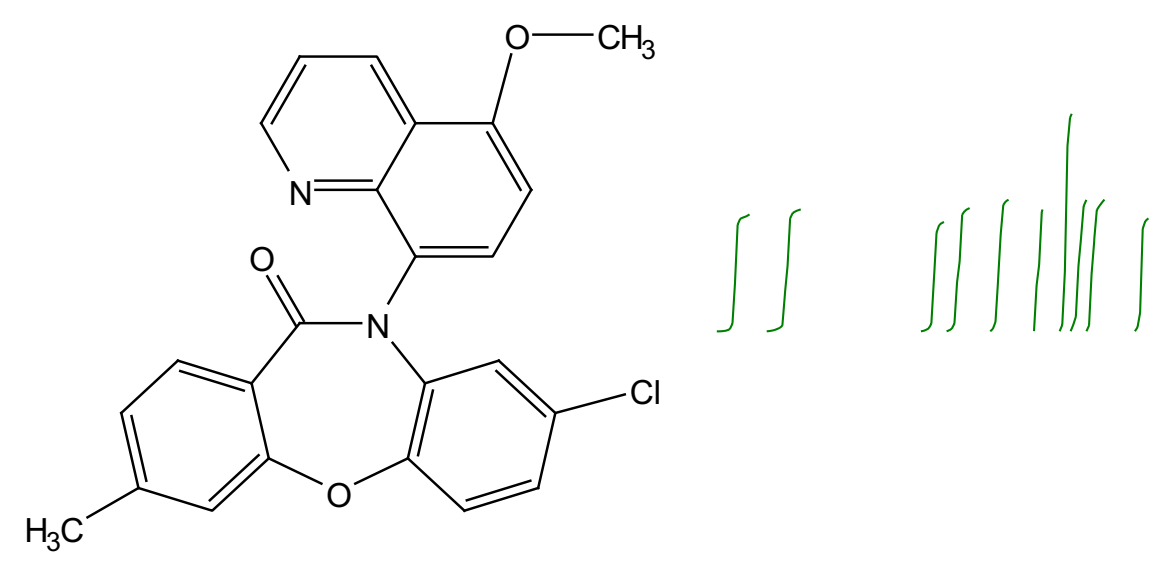

7lb

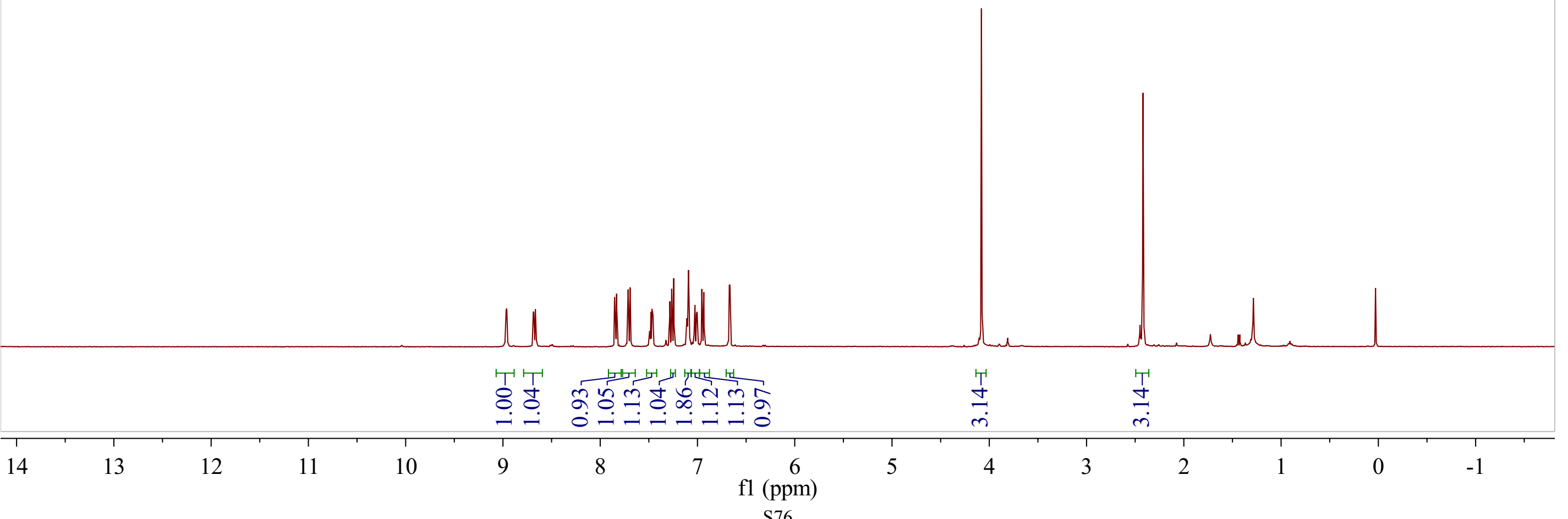


<smiles>COc1ccc(N2C(=O)c3ccc(C)cc3Oc3ccc(Cl)cc32)c2ncccc12</smiles>

7lb
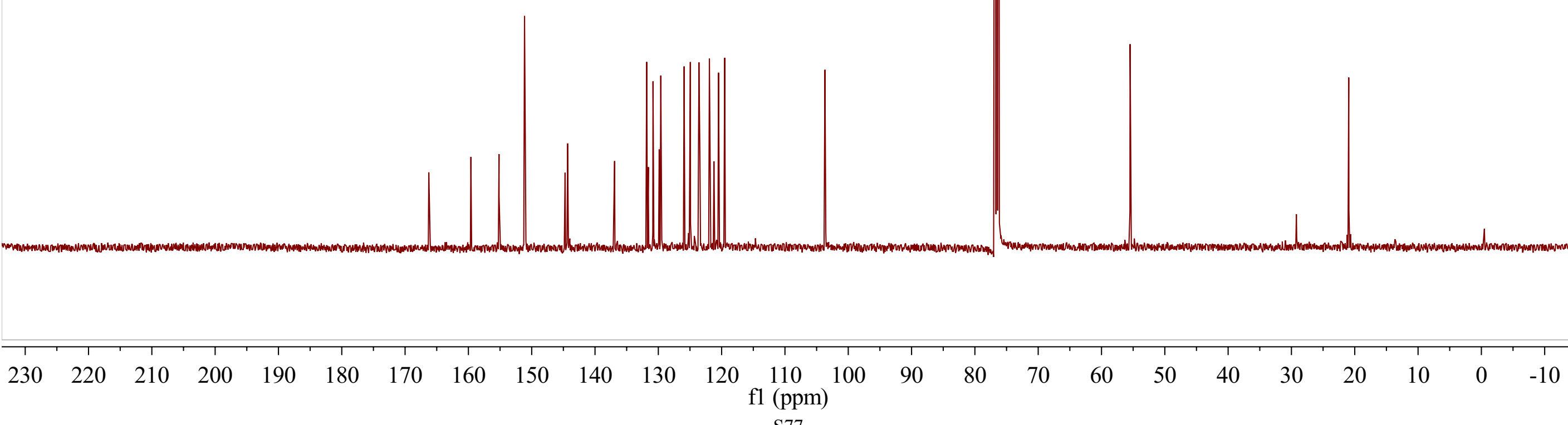


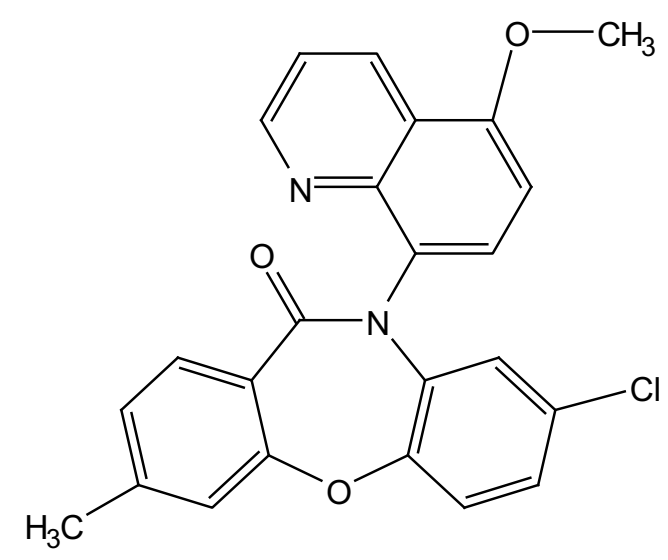

7lb

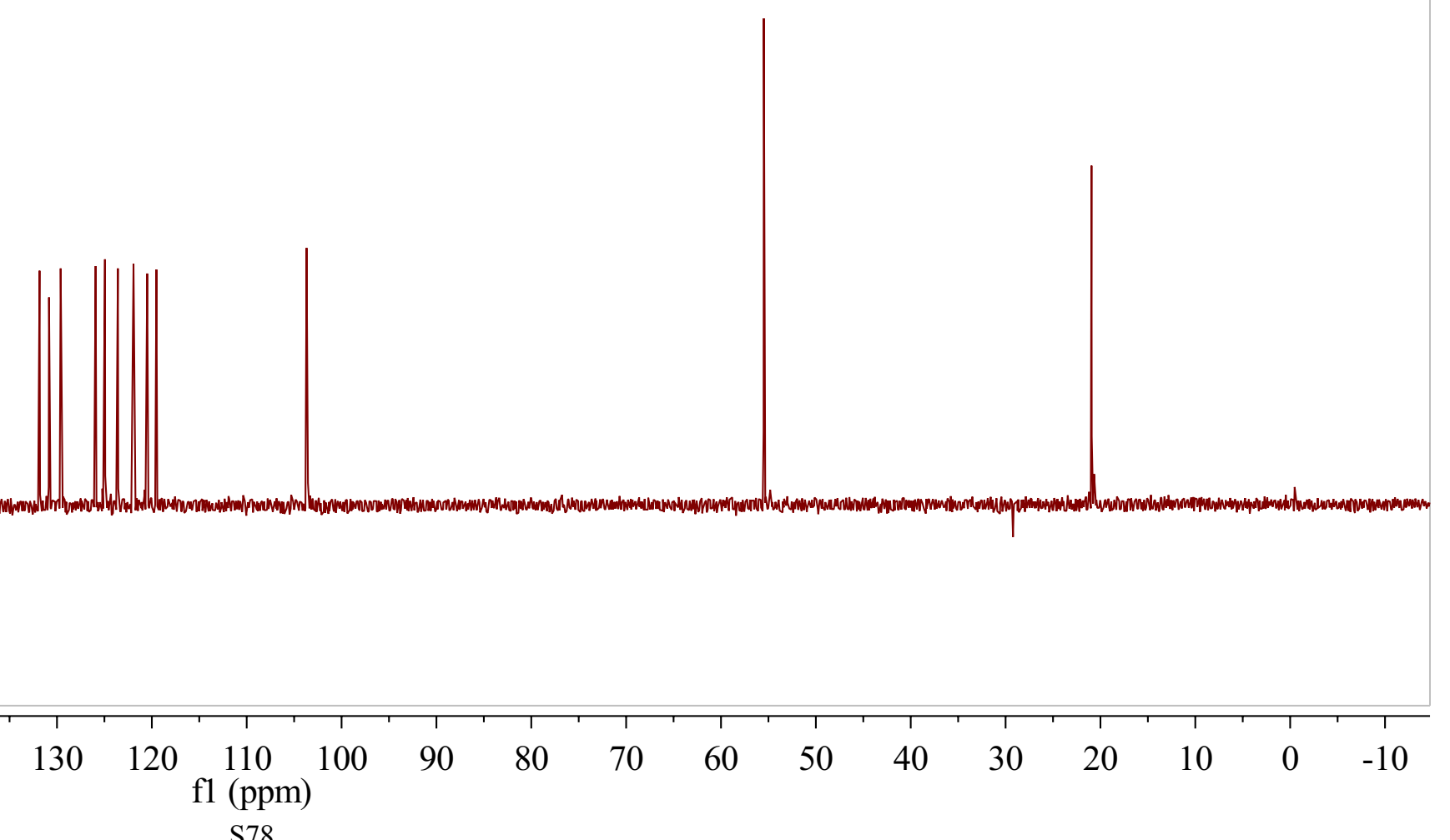



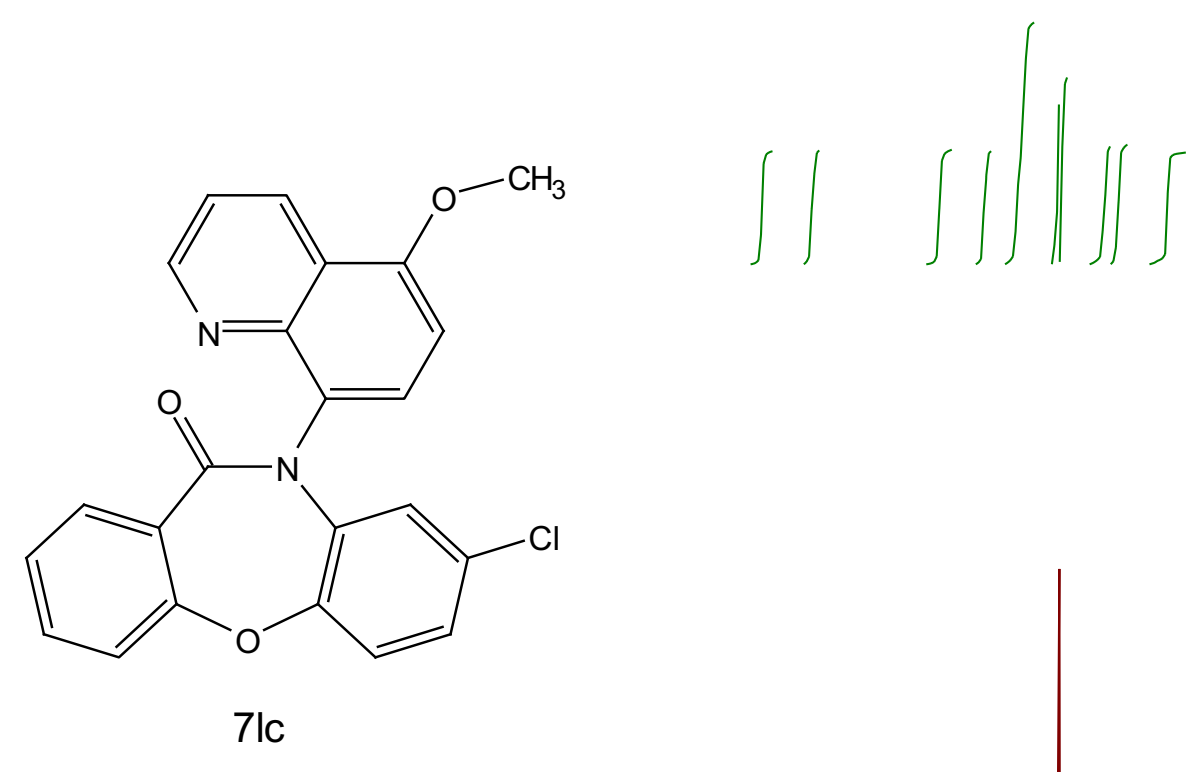

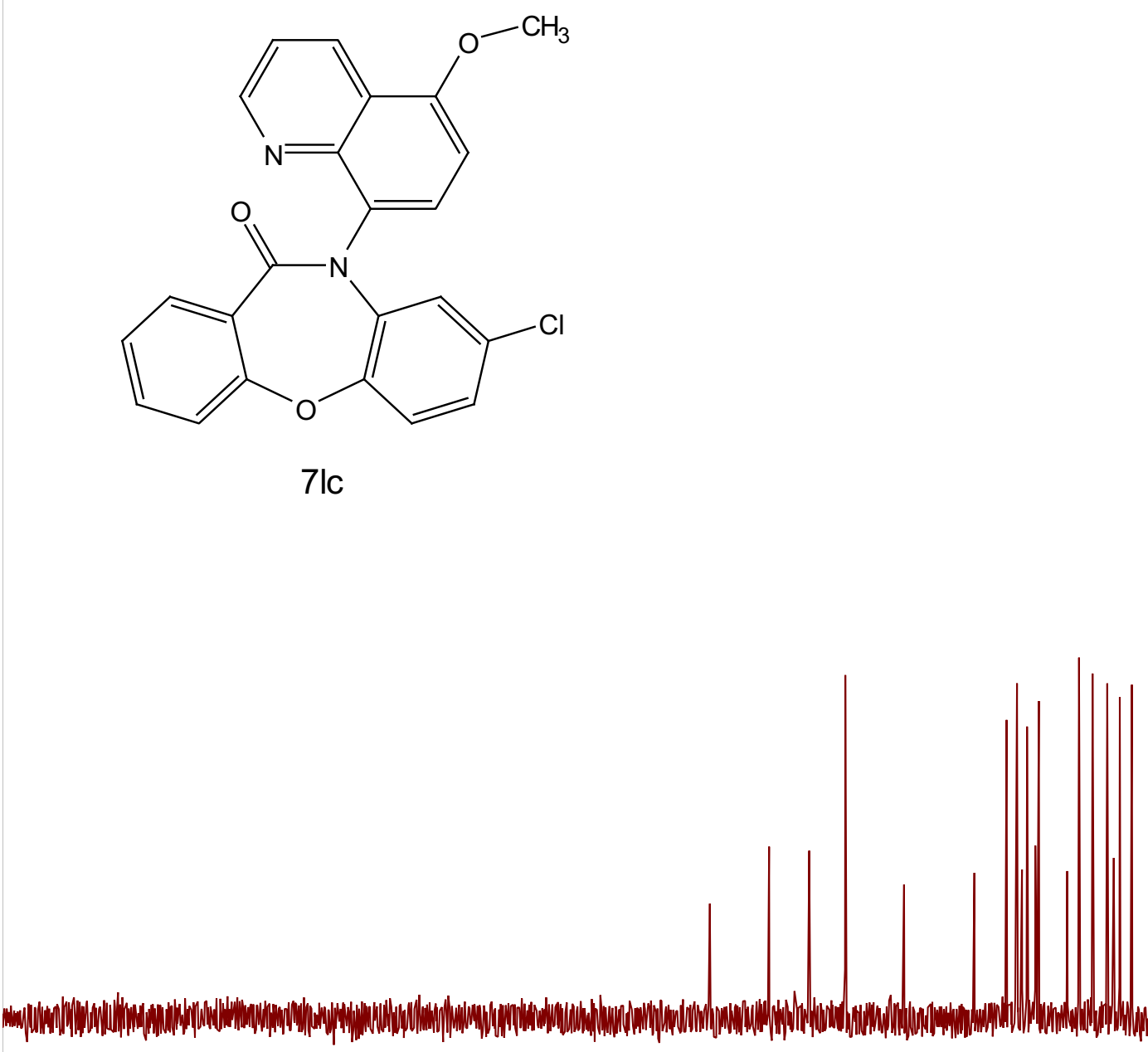

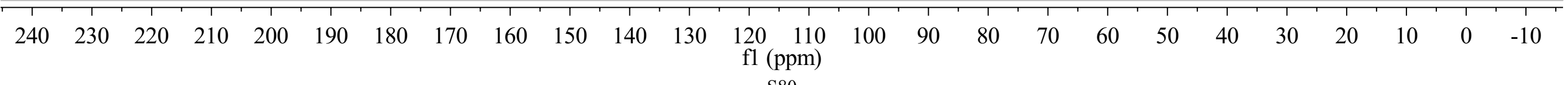




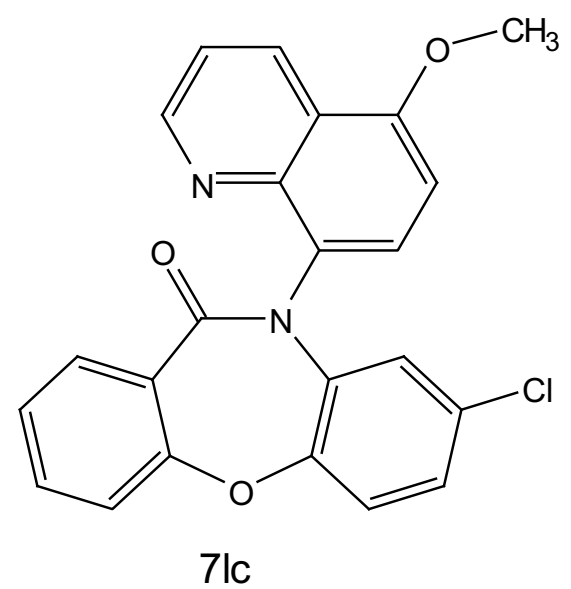

7lc

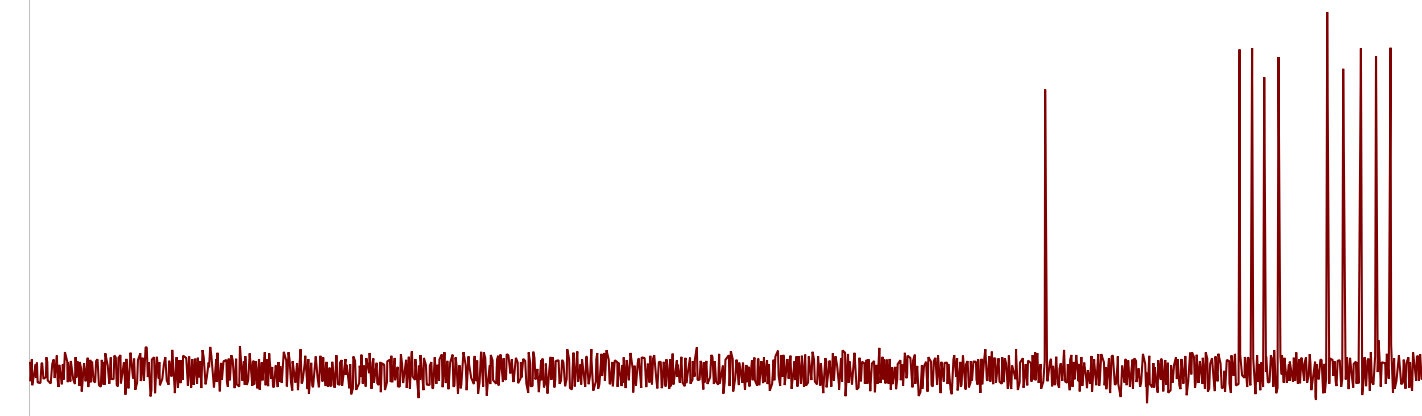

$\begin{array}{rllllllllllllllllllllllllll}240 & 230 & 220 & 210 & 200 & 190 & 180 & 170 & 160 & 150 & 140 & 130 & 120 & 110 & 100 & 90 & 80 & 70 & 60 & 50 & 40 & 30 & 20 & 10 & 0 & -10\end{array}$



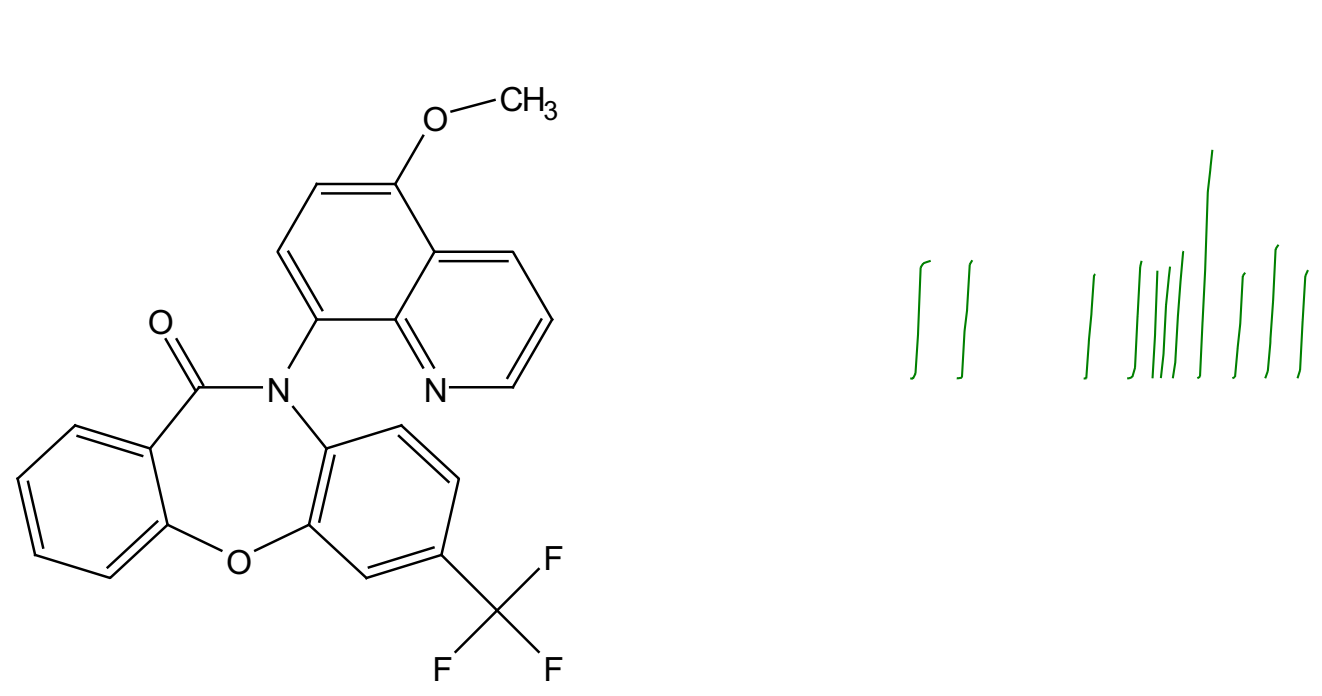

7ld

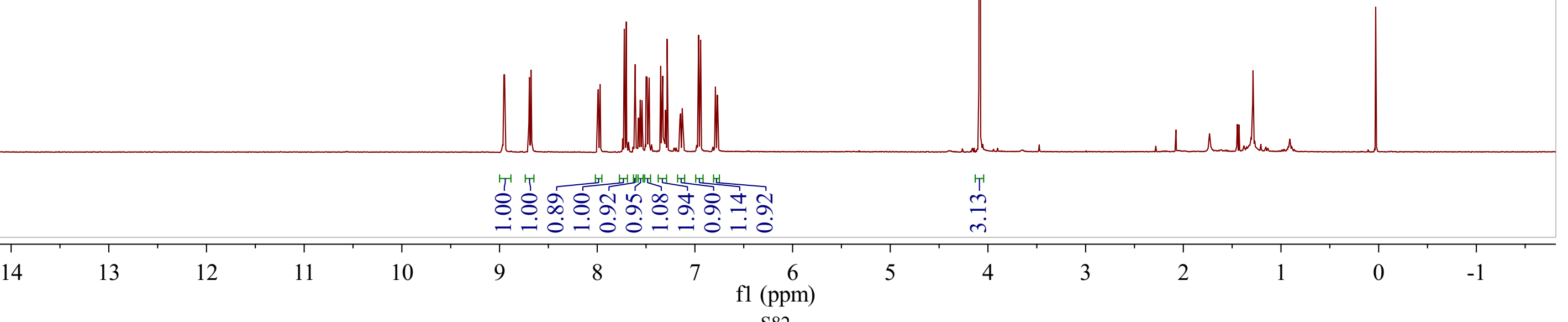



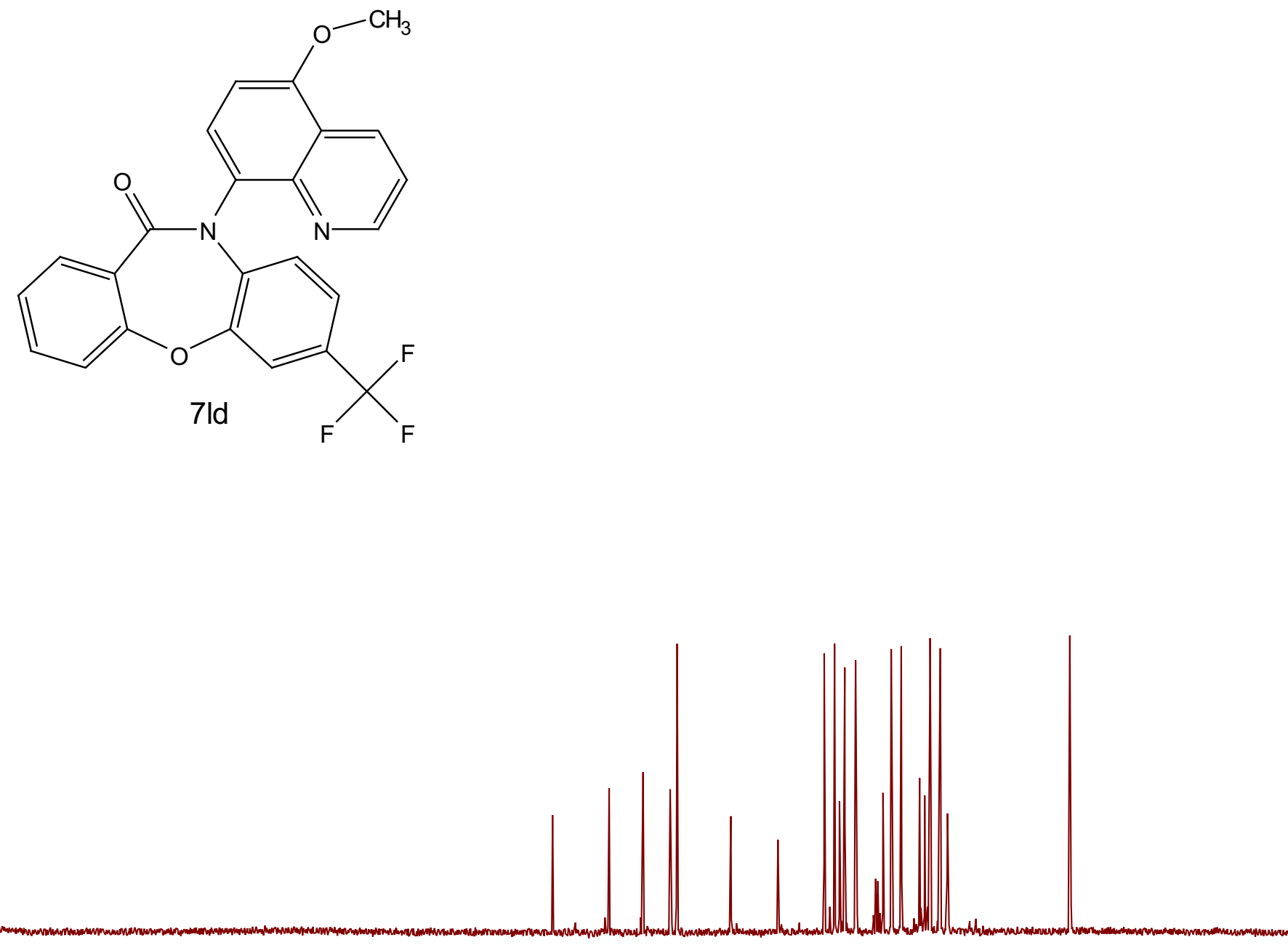


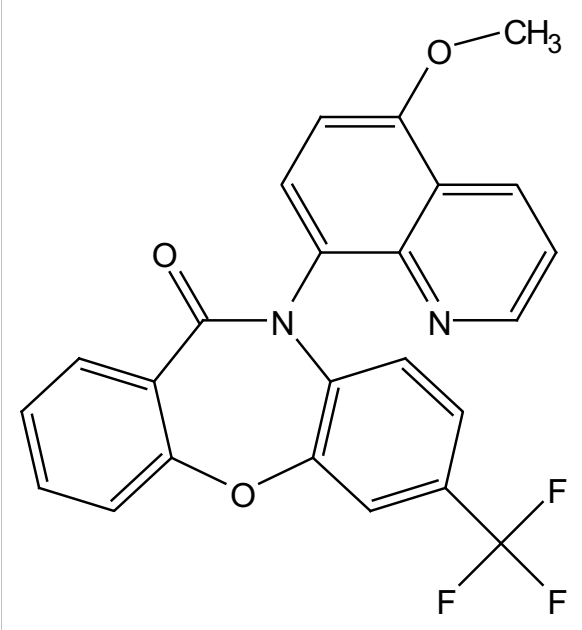

7ld

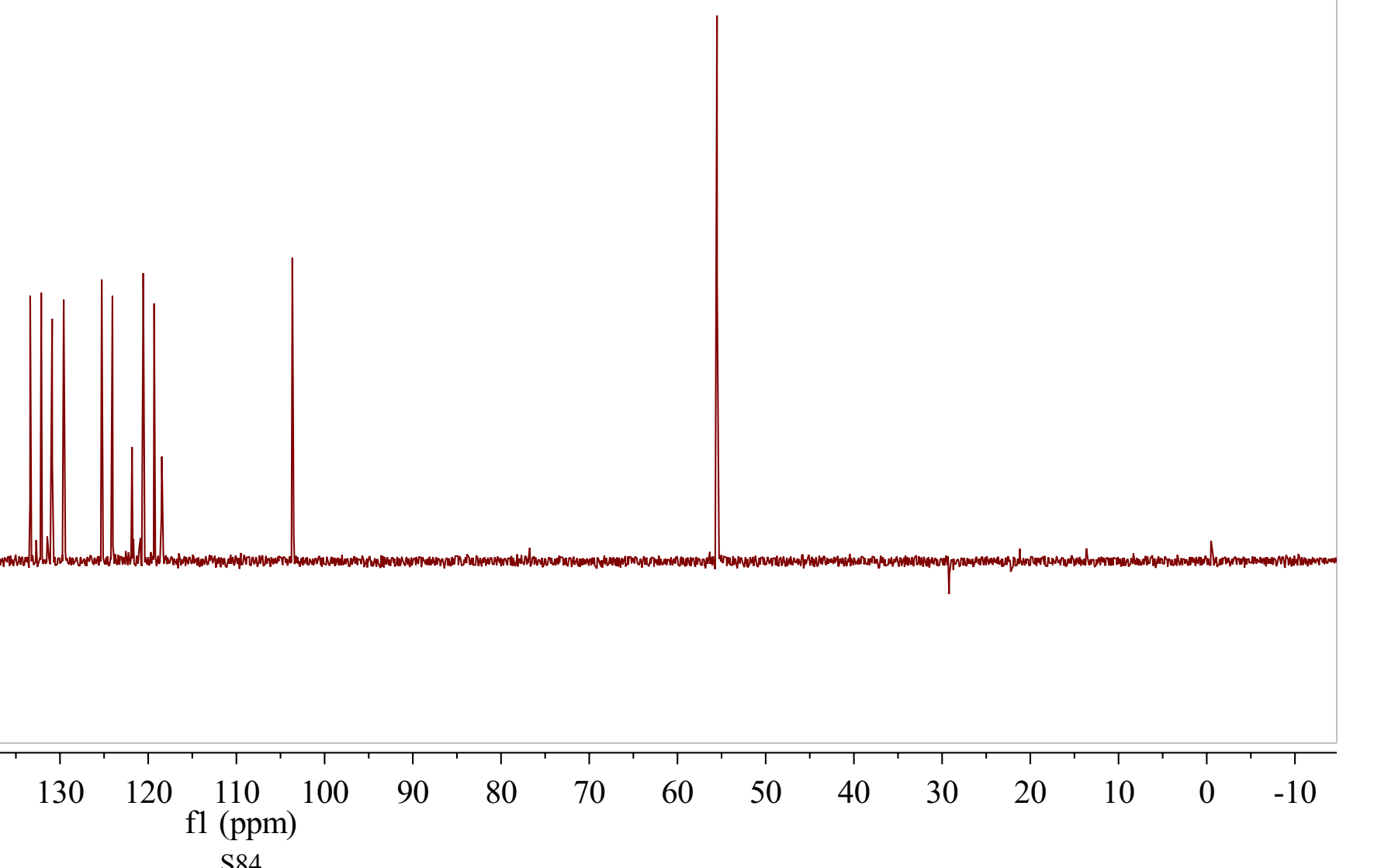




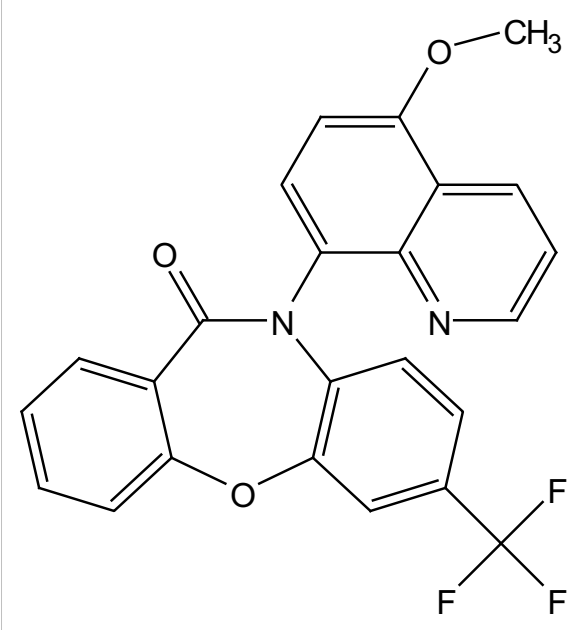

7ld

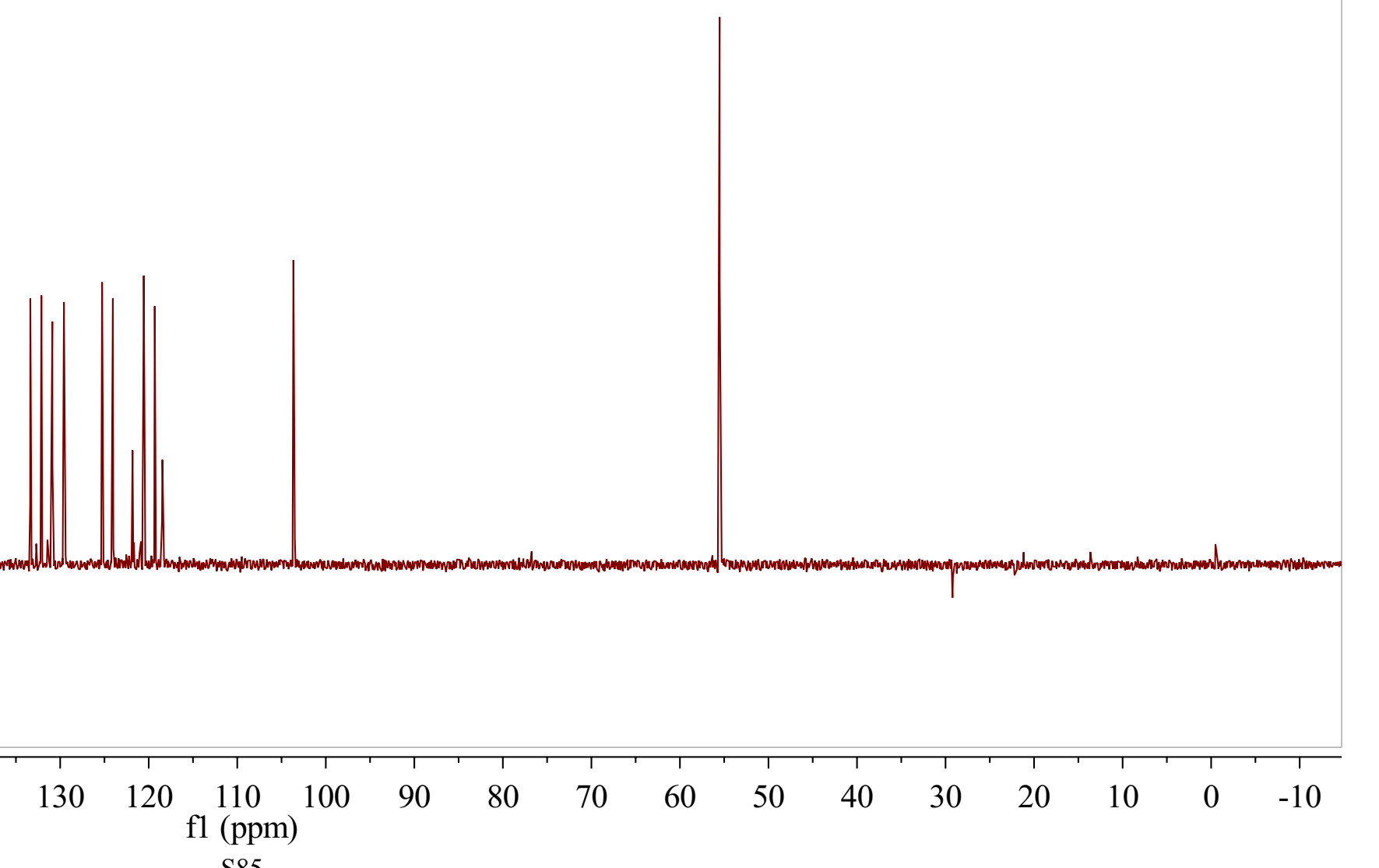



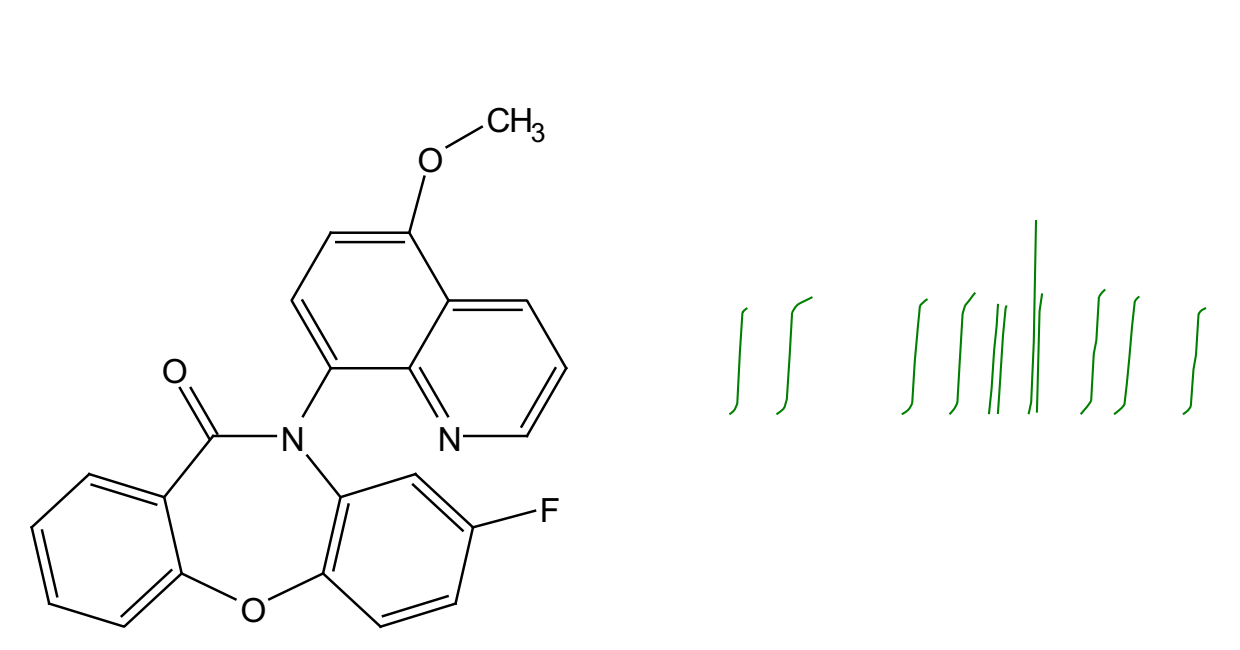

7le
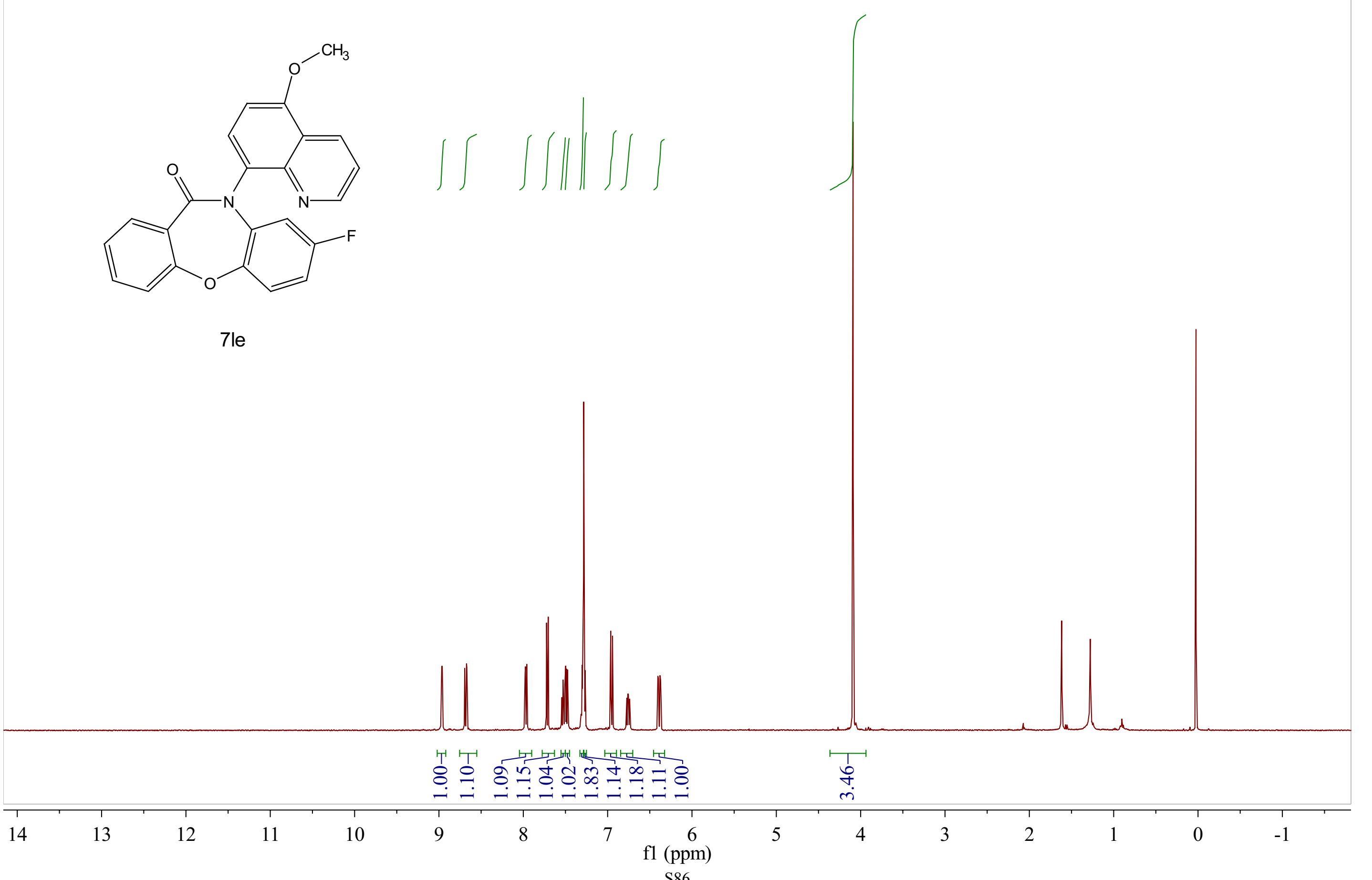


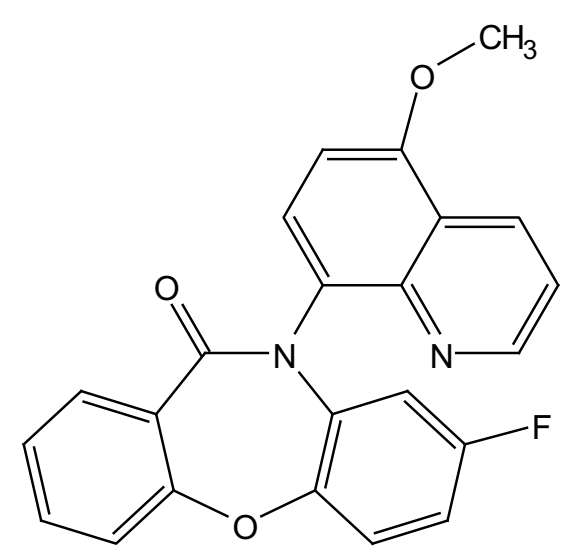

$7 l e$

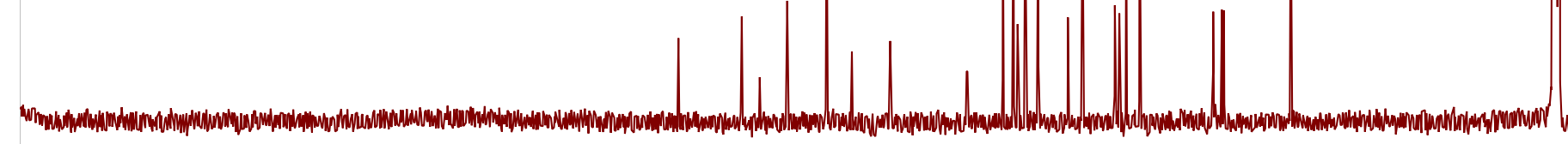

$\begin{array}{lllllllllllllllllllllllll}230 & 220 & 210 & 200 & 190 & 180 & 170 & 160 & 150 & 140 & 130 & 120 & \begin{array}{l}110 \\ \text { fl (ppm) }\end{array} & 100 & 90 & 80 & 70 & 60 & 50 & 40 & 30 & 20 & 10 & 0 & -10\end{array}$




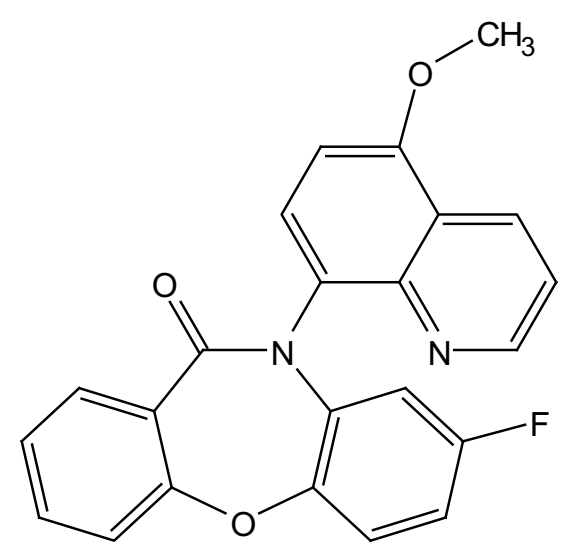

$7 l e$

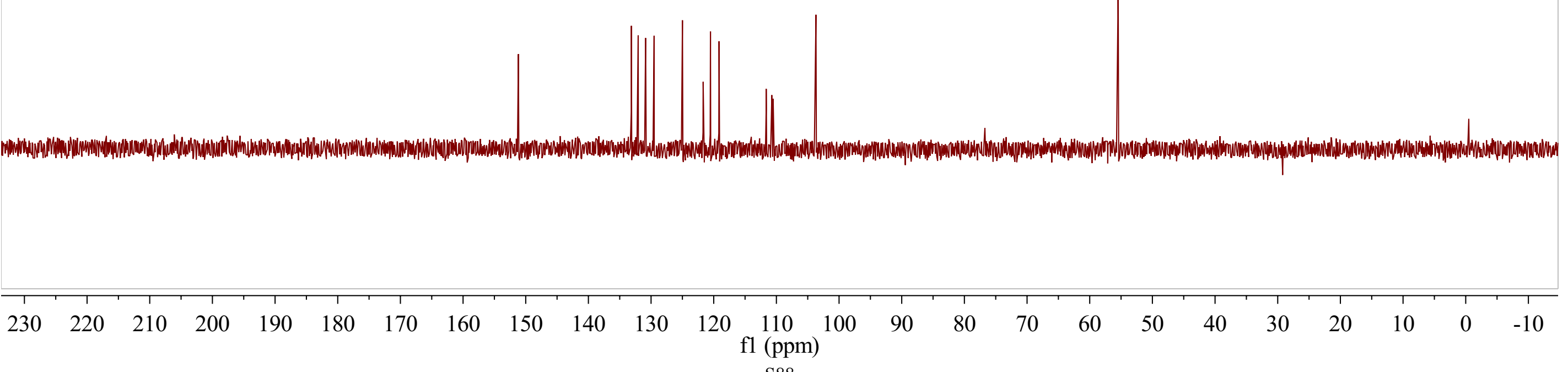



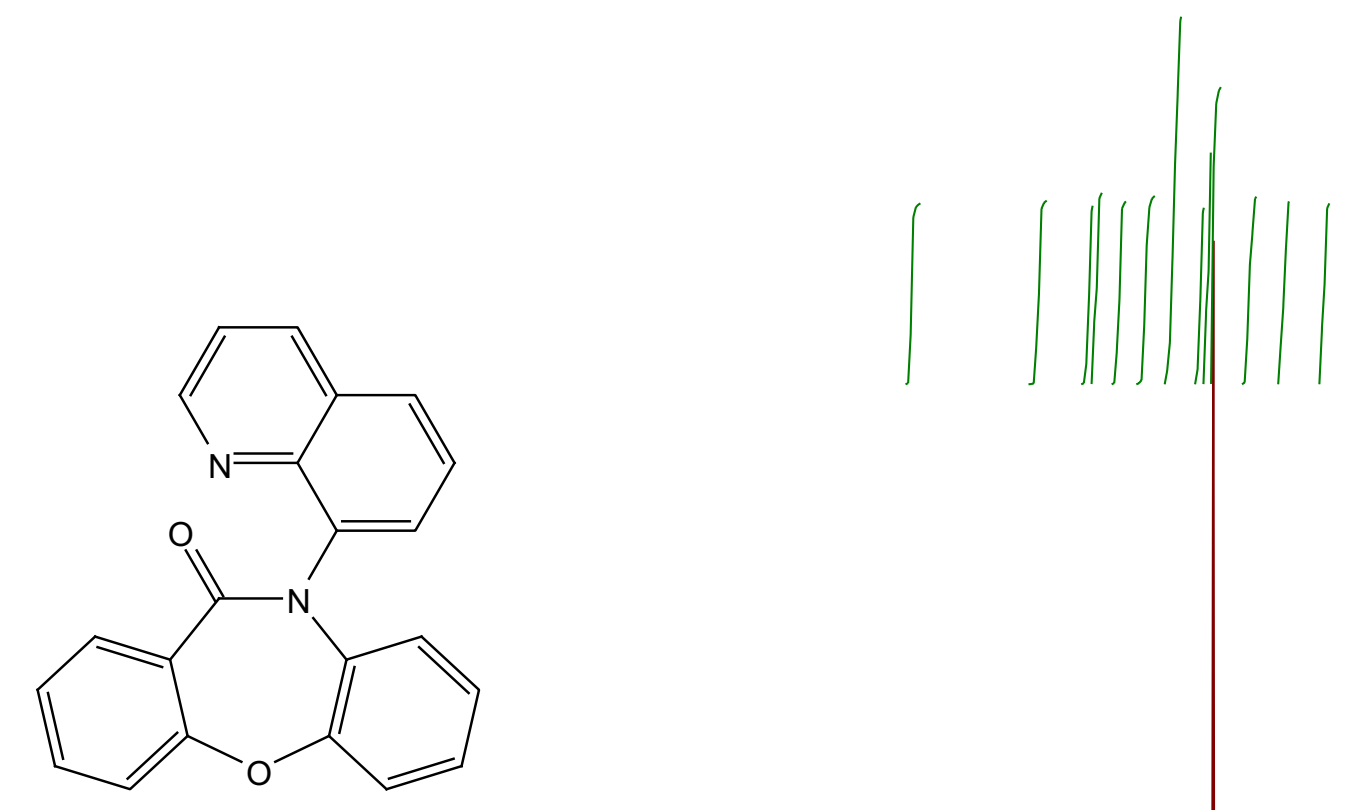

70

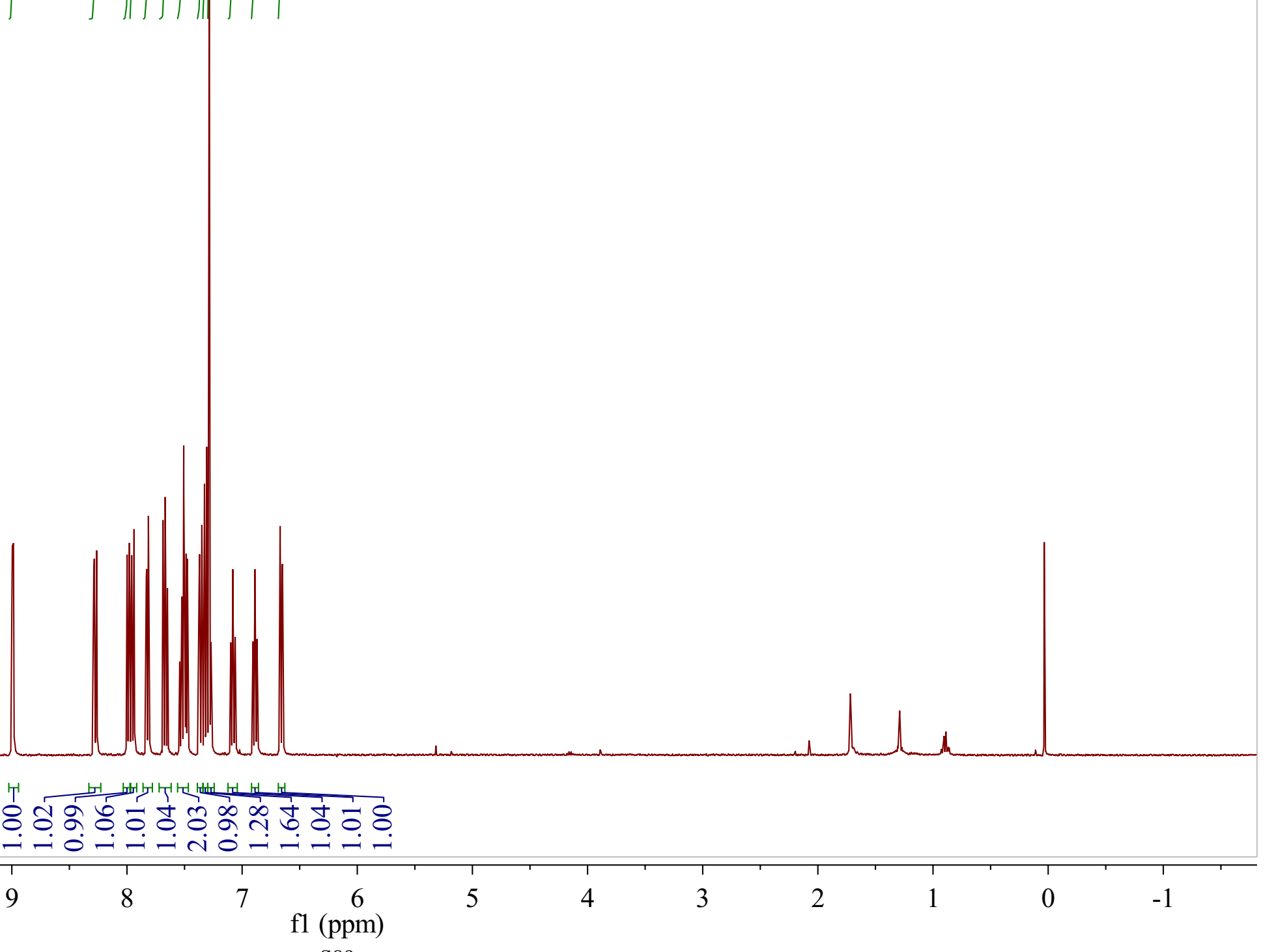




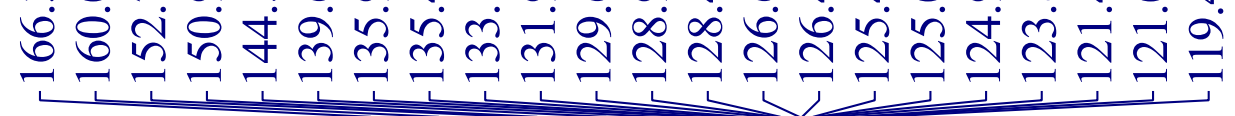

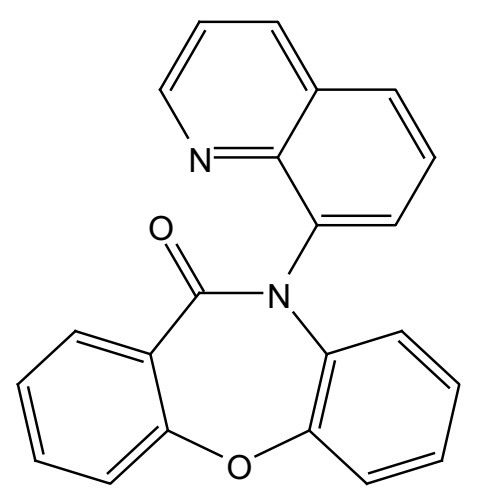

70

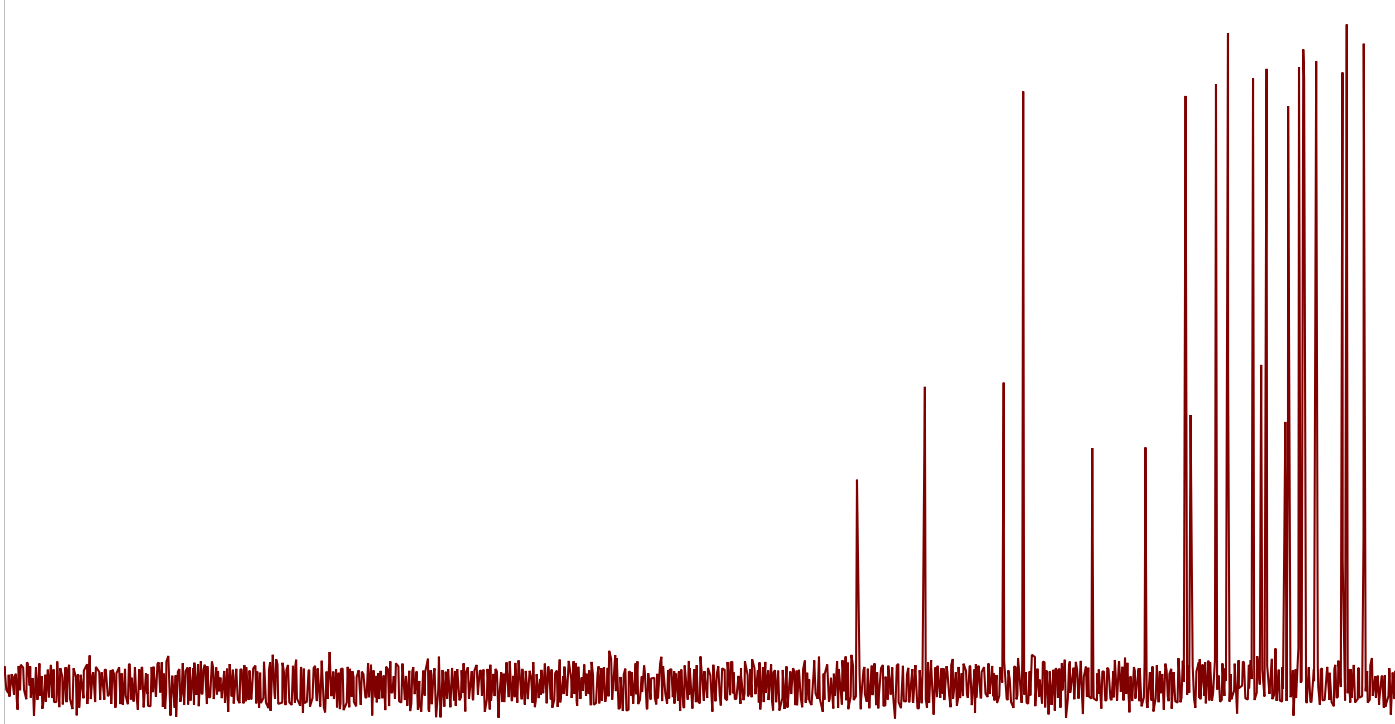

\begin{tabular}{lllllllllllllllllllllllllll}
\hline 240 & 230 & 220 & 210 & 200 & 190 & 180 & 170 & 160 & 150 & 140 & 130 & $\begin{array}{c}120 \\
\mathrm{fl}(\mathrm{ppm})\end{array}$ & 100 & 90 & 80 & 70 & 60 & 50 & 40 & 30 & 20 & 10 & 0 & -10 & &
\end{tabular}



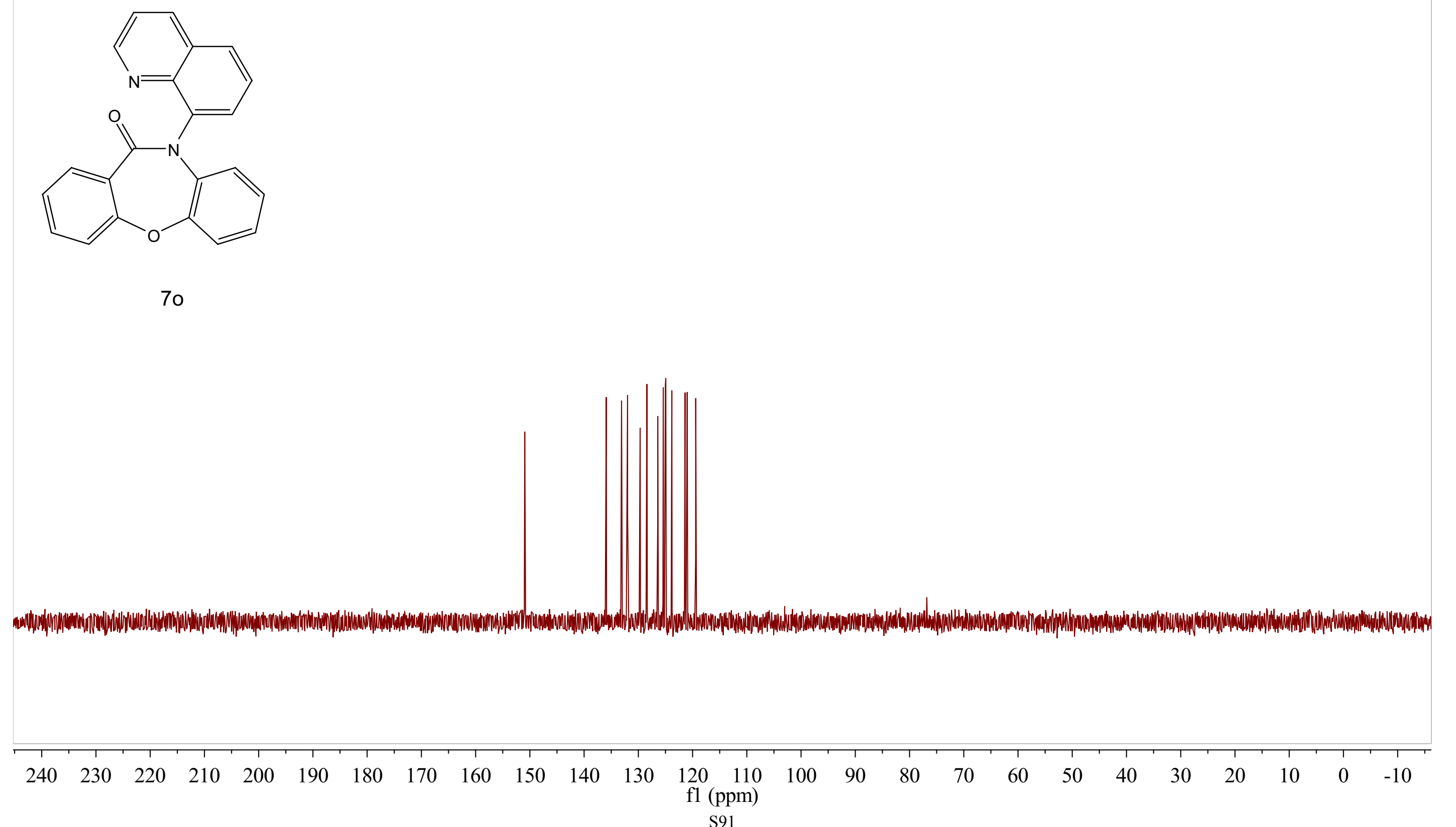

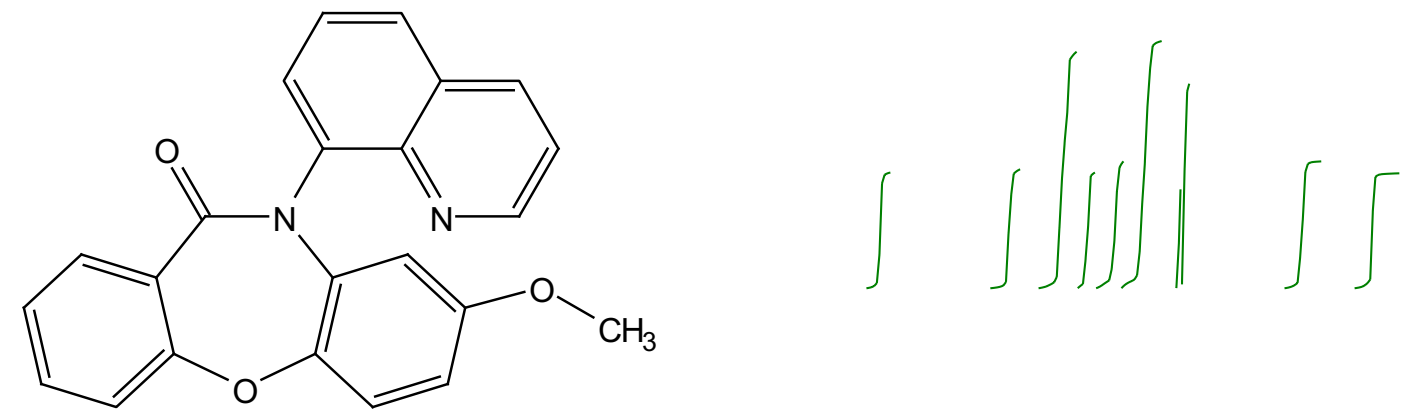

$7 p$

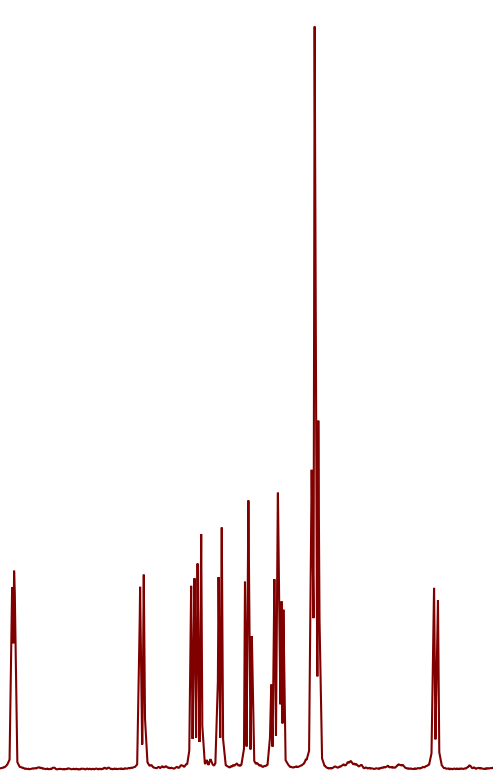

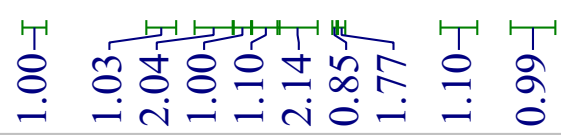

$\stackrel{\frac{1}{\infty}}{\frac{1}{m}}$

13

12

11

10

9

8

$7 \quad \begin{array}{cc}6 \\ \text { fl }(\mathrm{pbm})\end{array}$

S92 


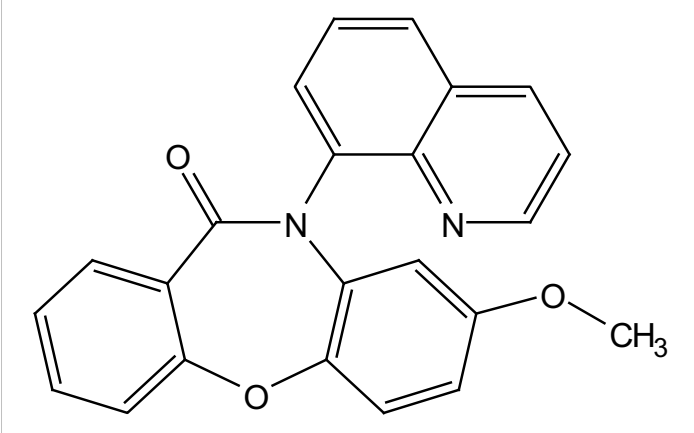

$7 p$

\begin{tabular}{llllllllllllllllllllllllllllll}
\hline 230 & 220 & 210 & 200 & 190 & 180 & 170 & 160 & 150 & 140 & 130 & 120 & 110 & 100 & 90 & 80 & 70 & 60 & 50 & 40 & 30 & 20 & 10 & 0 & -10 &
\end{tabular}




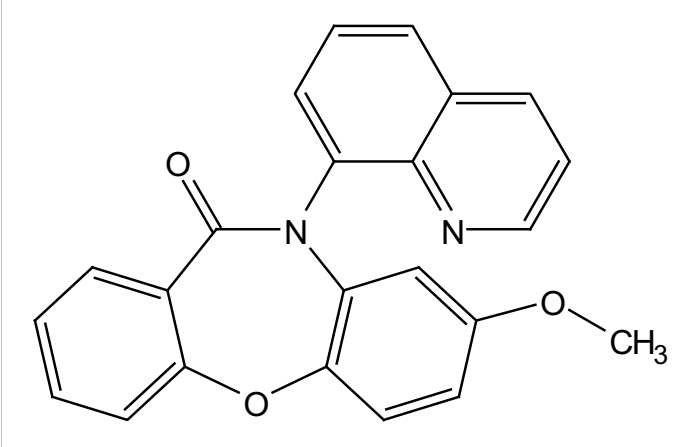

$7 p$

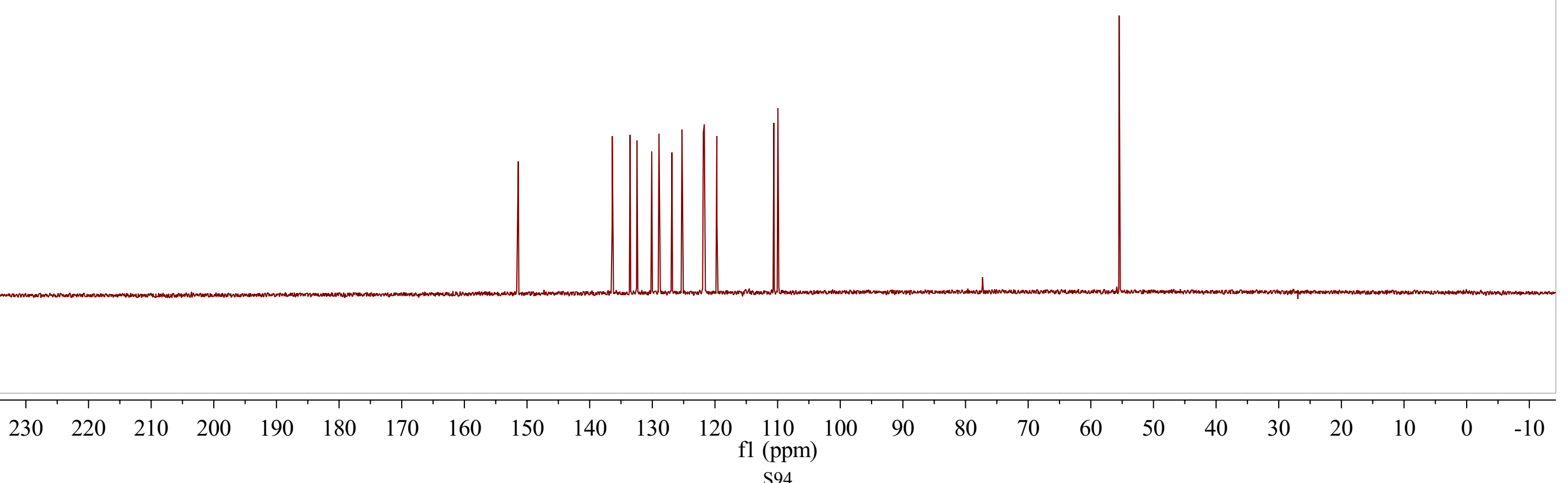



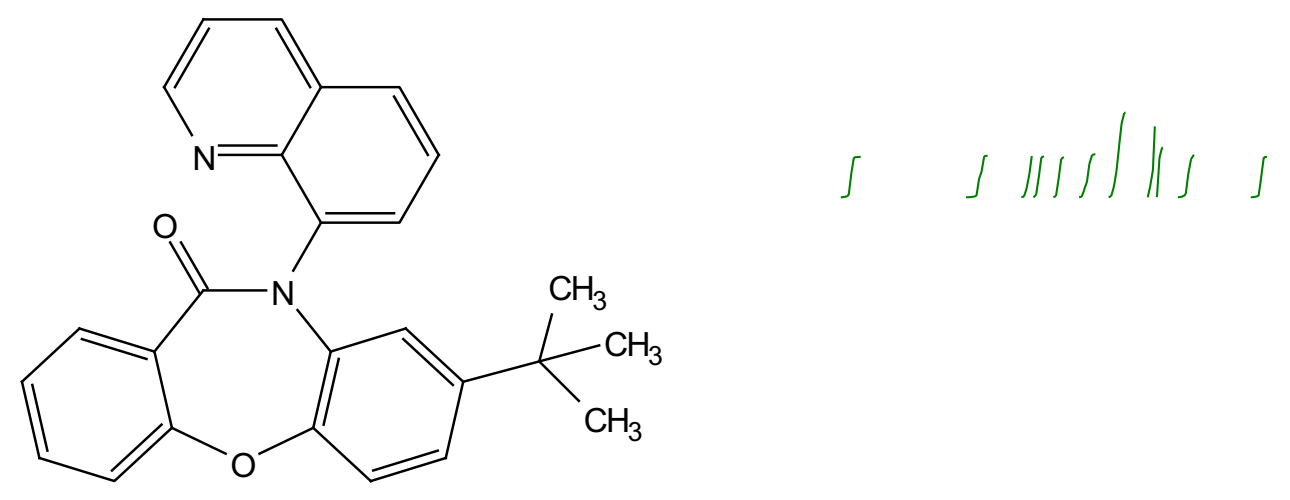

$7 q$

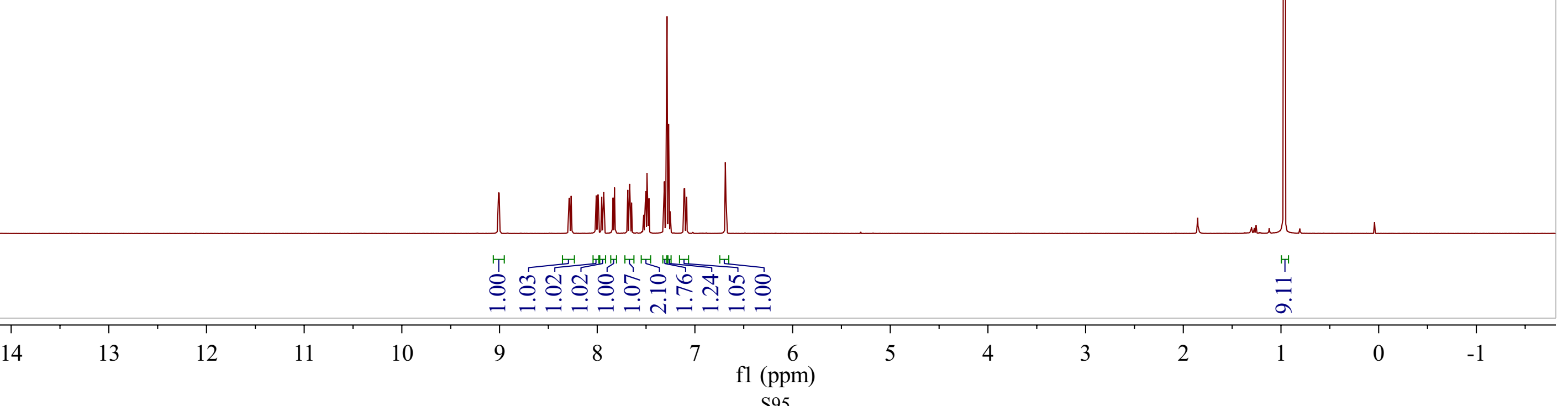




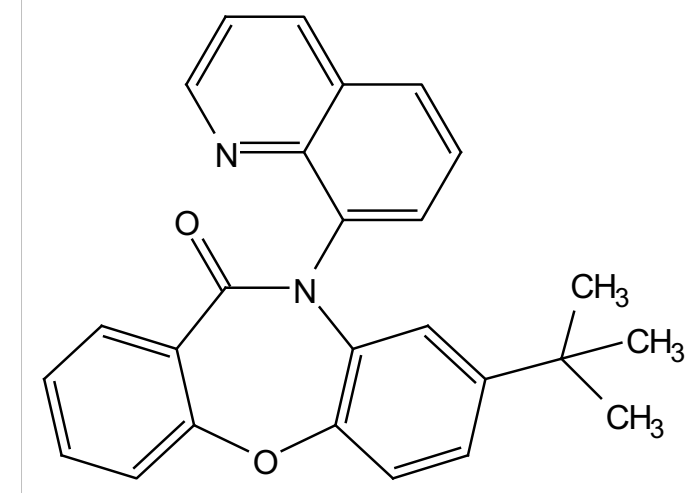

$7 q$

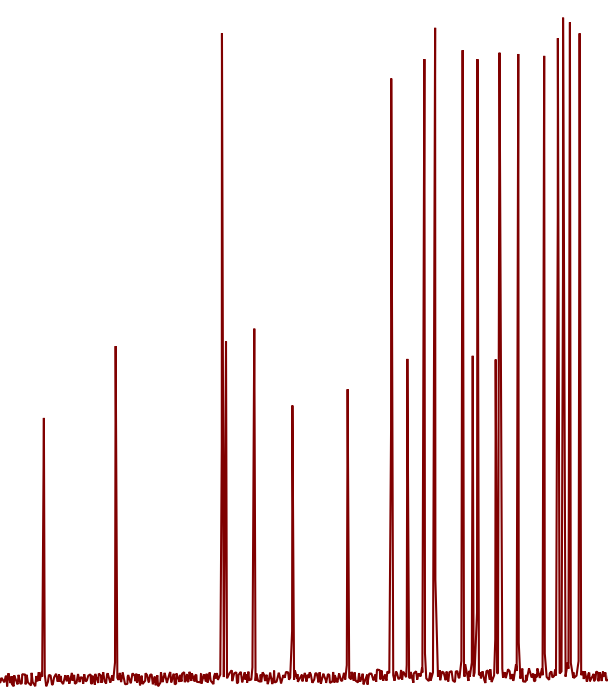

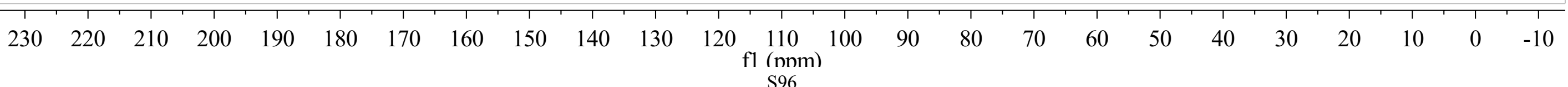




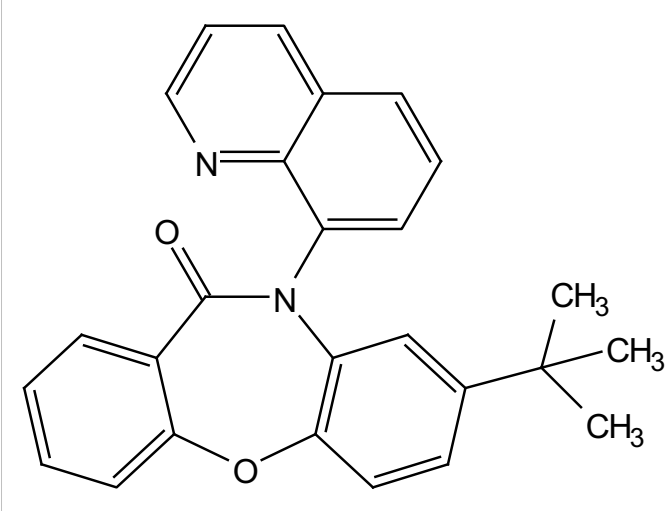

$7 q$

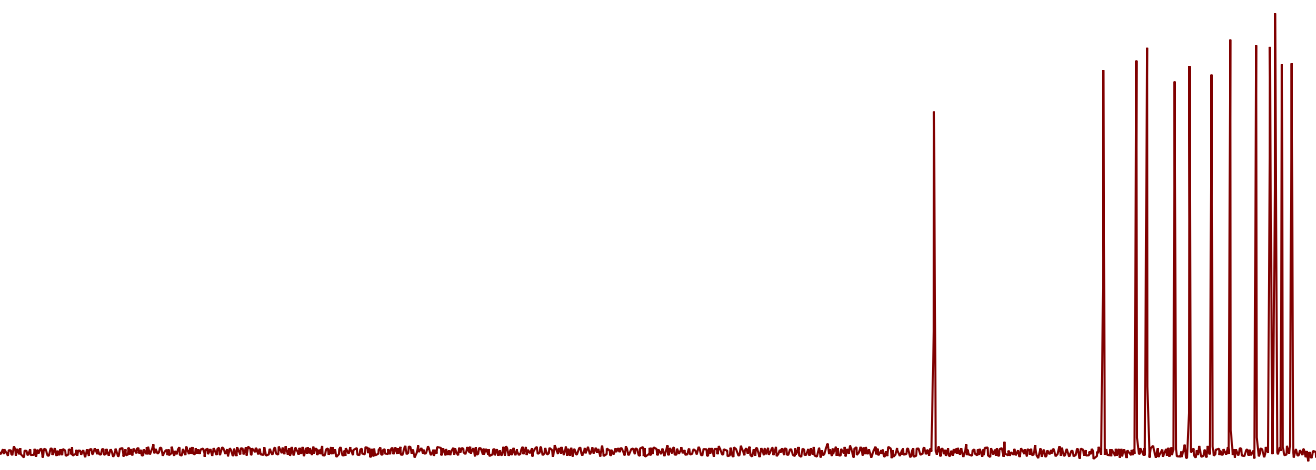

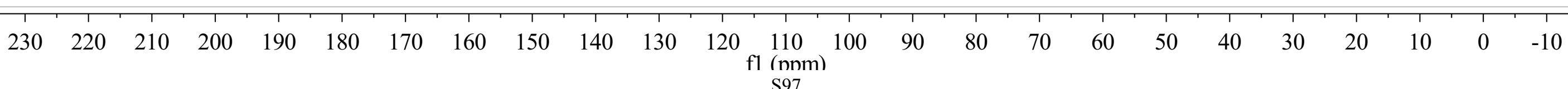



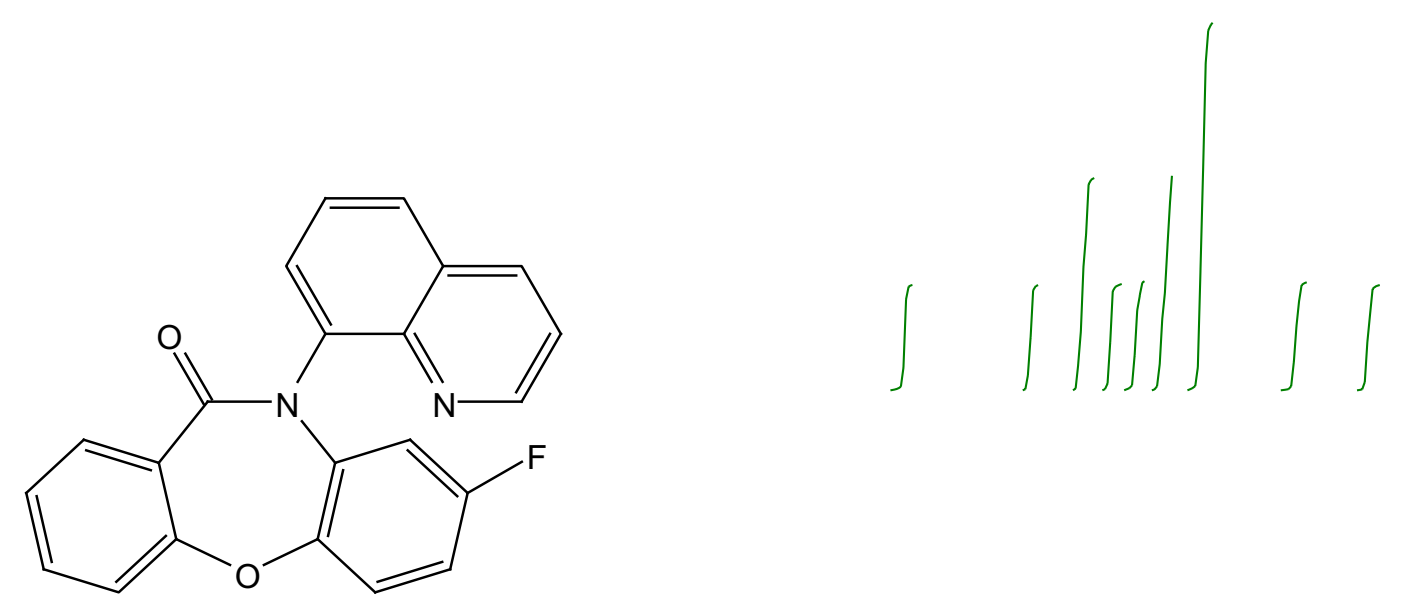

$7 r$

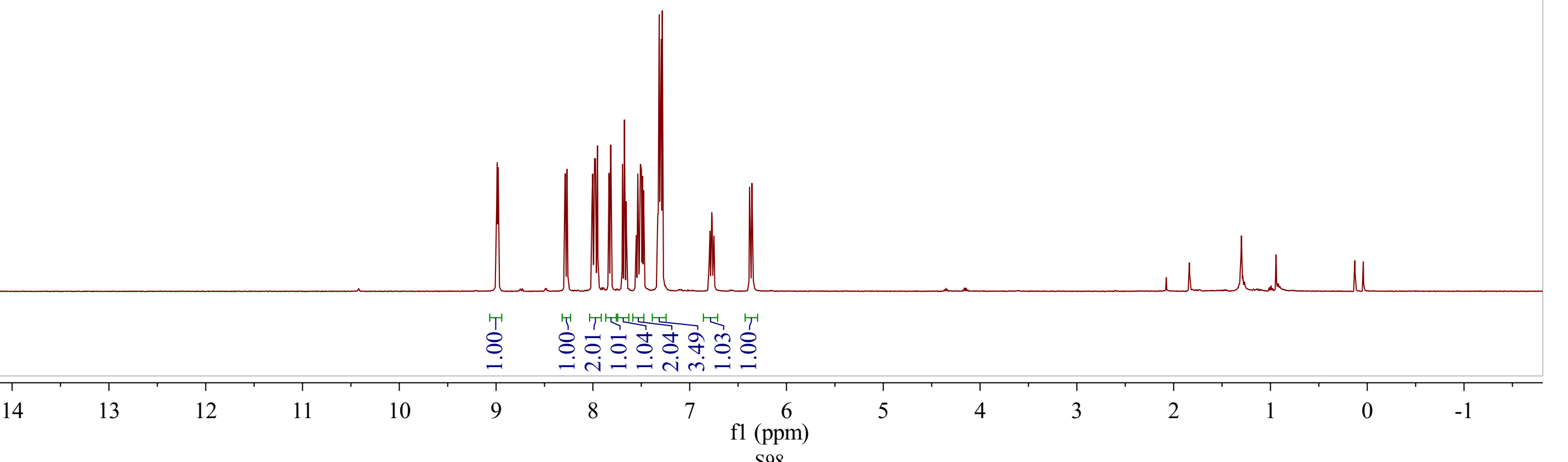




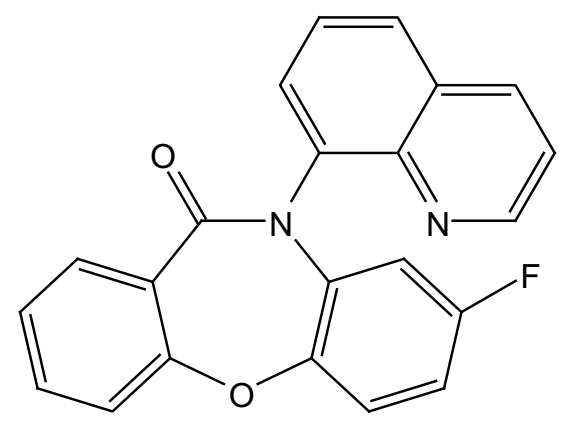

$7 r$

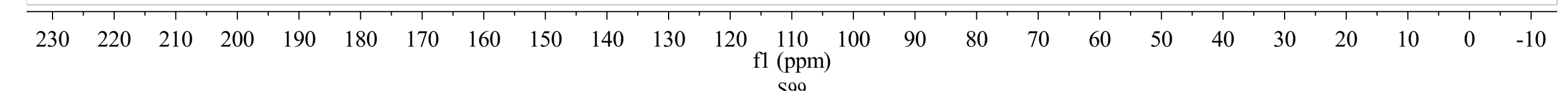




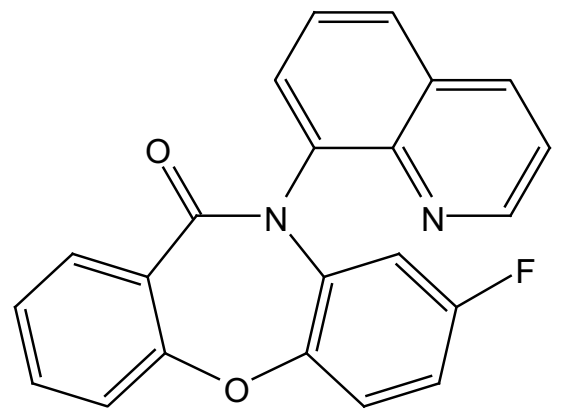

$7 r$

\begin{tabular}{lllllllllllllllllllllllll}
\hline 230 & 220 & 210 & 200 & 190 & 180 & 170 & 160 & 150 & 140 & 130 & 120 & 110 & 100 & 90 & 80 & 70 & 60 & 50 & 40 & 30 & 20 & 10 & 0 & -10
\end{tabular}



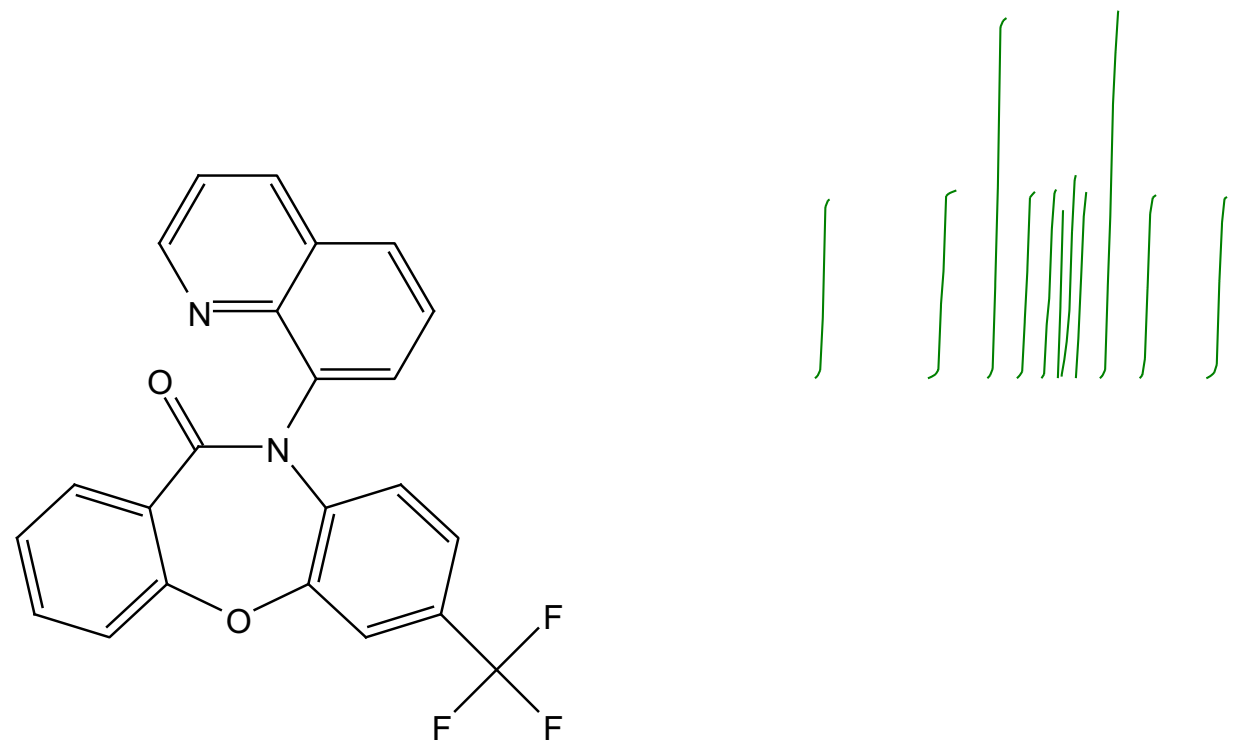

$7 s$

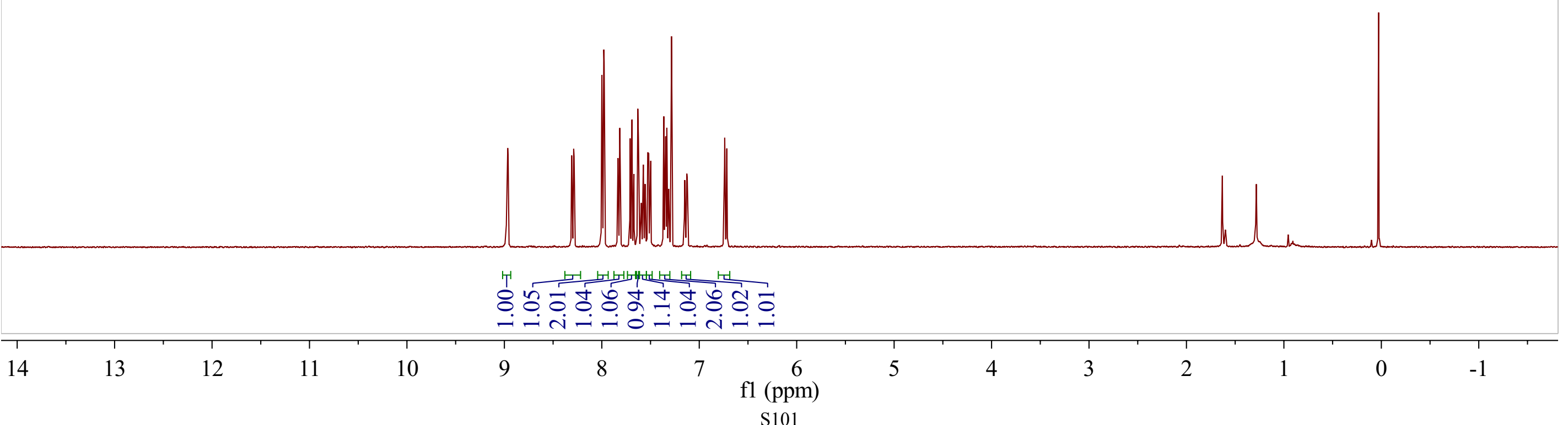




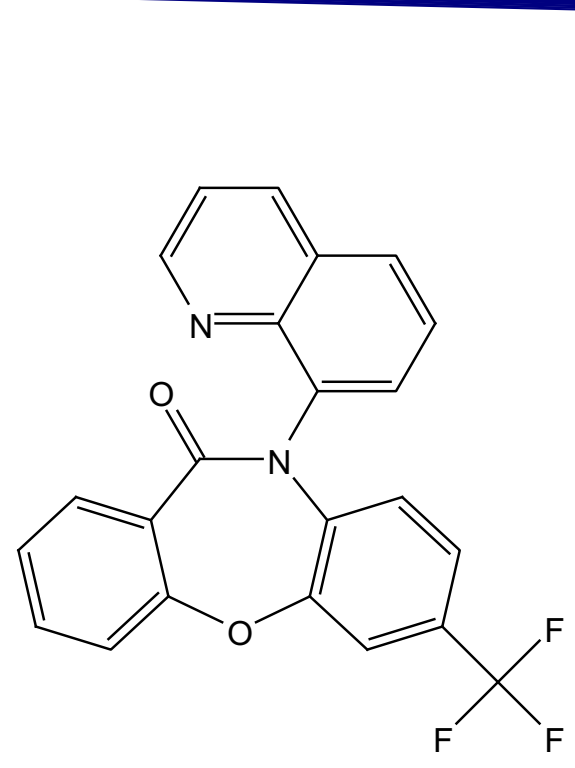

$7 \mathrm{~s}$

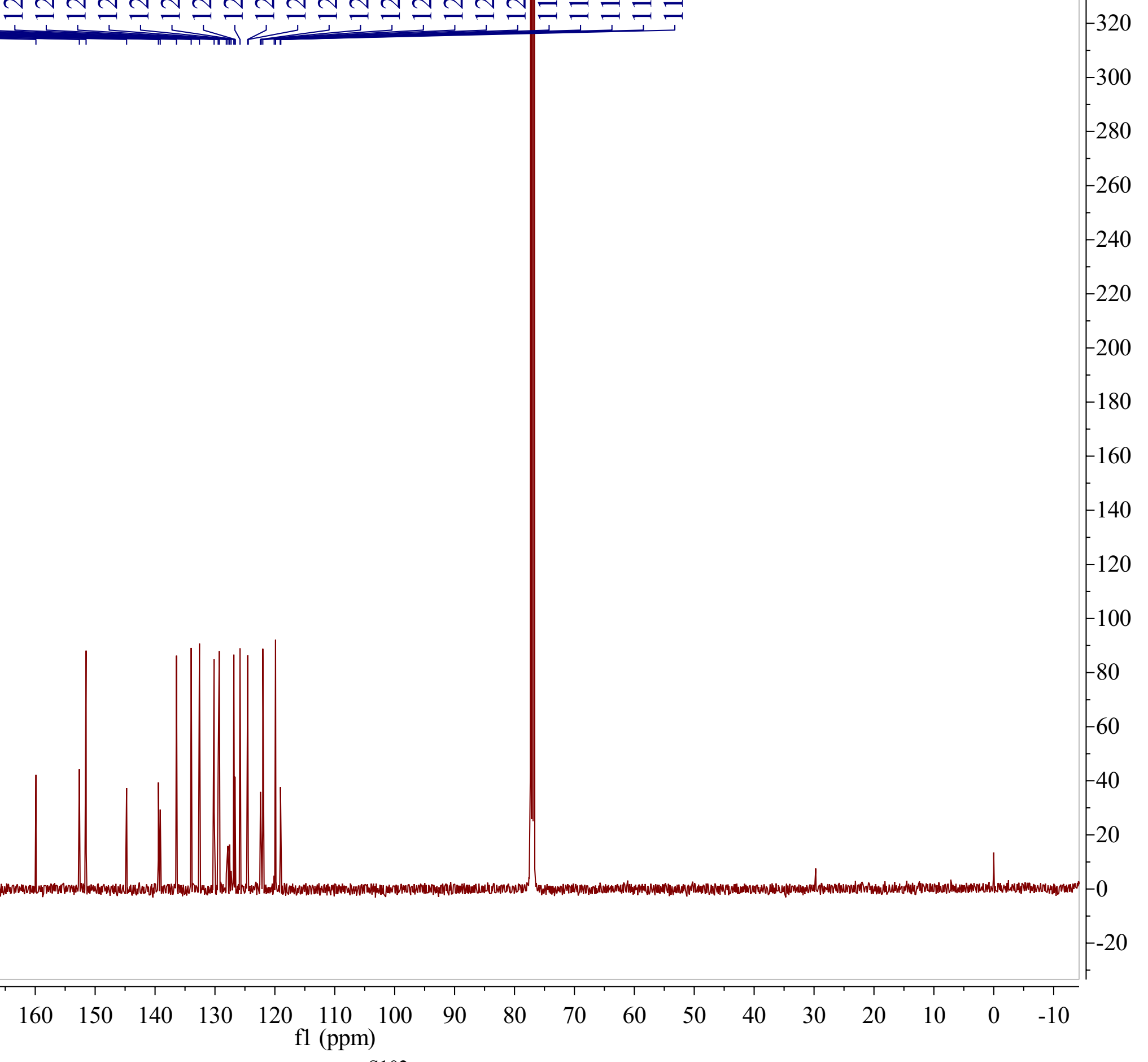




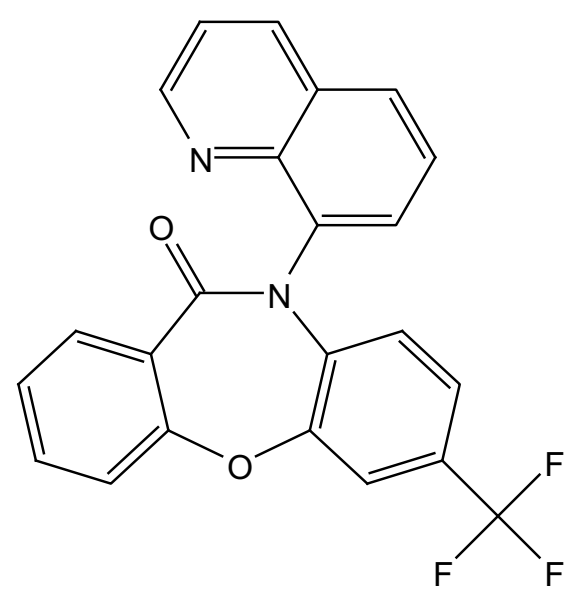

$7 s$

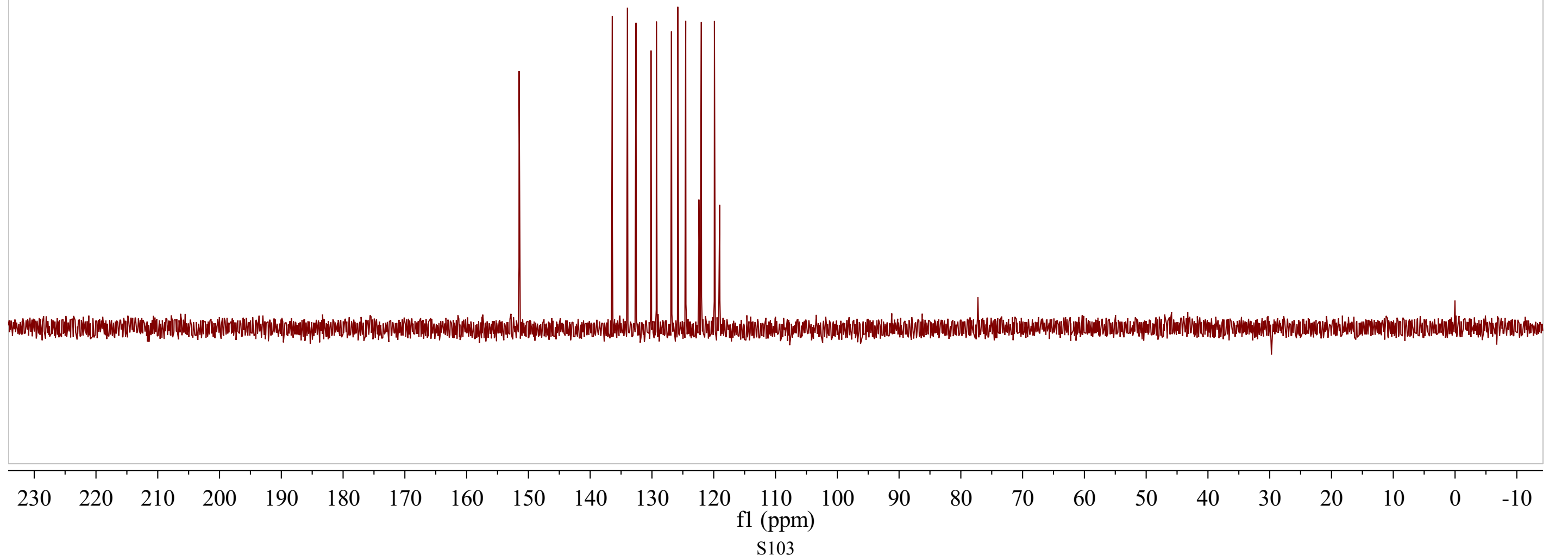



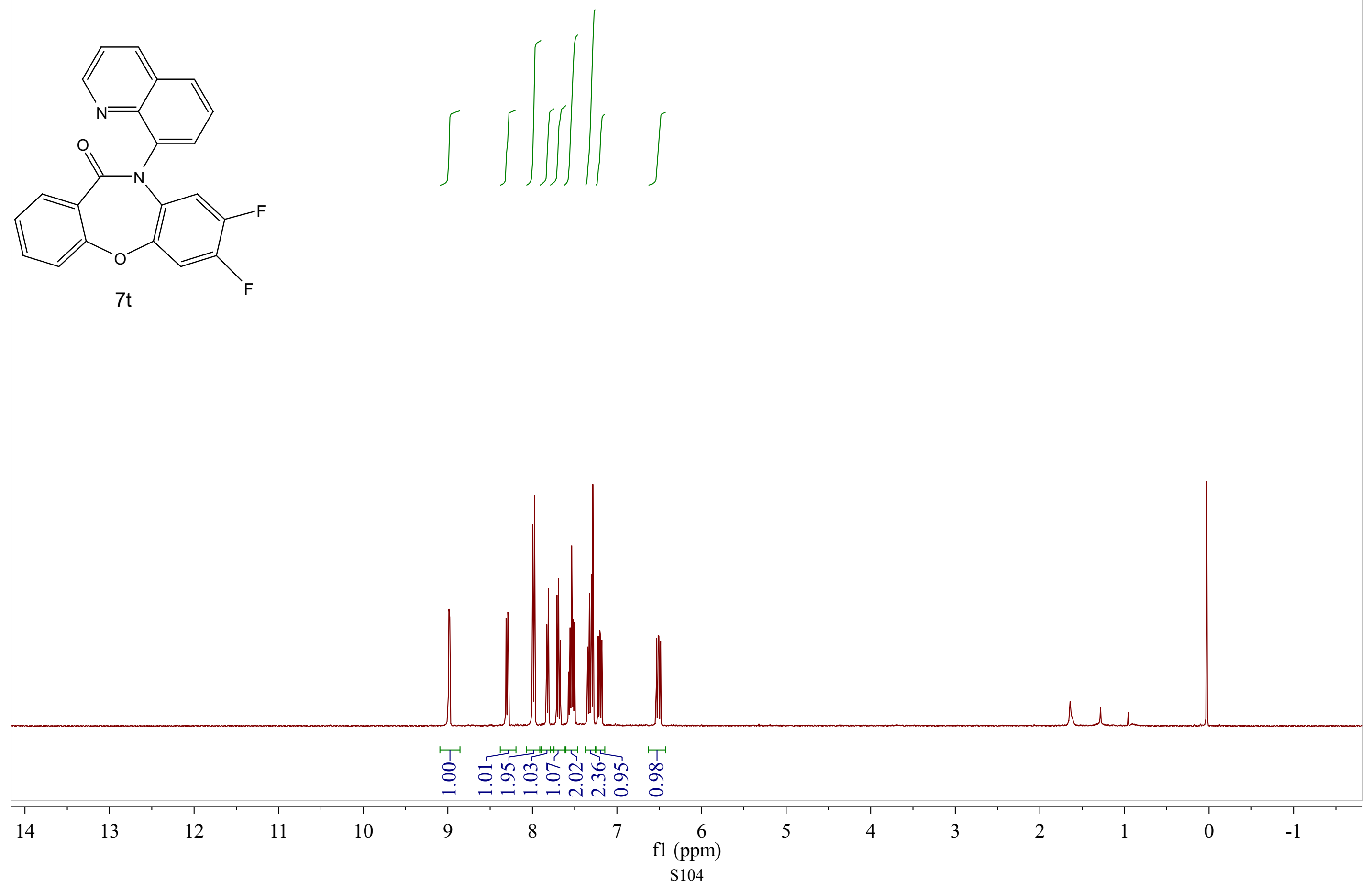


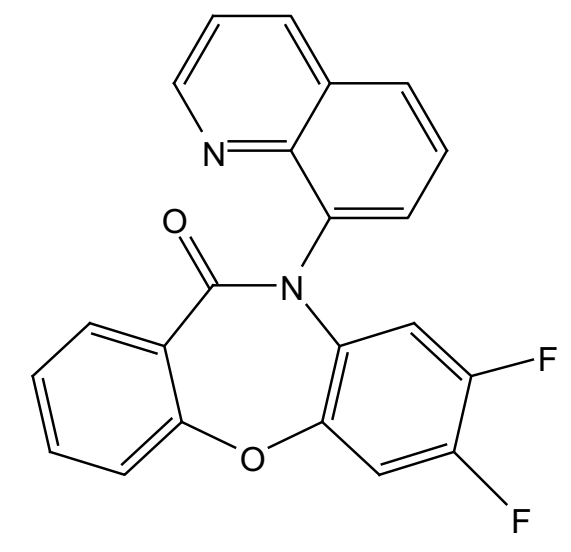

$7 \mathrm{t}$

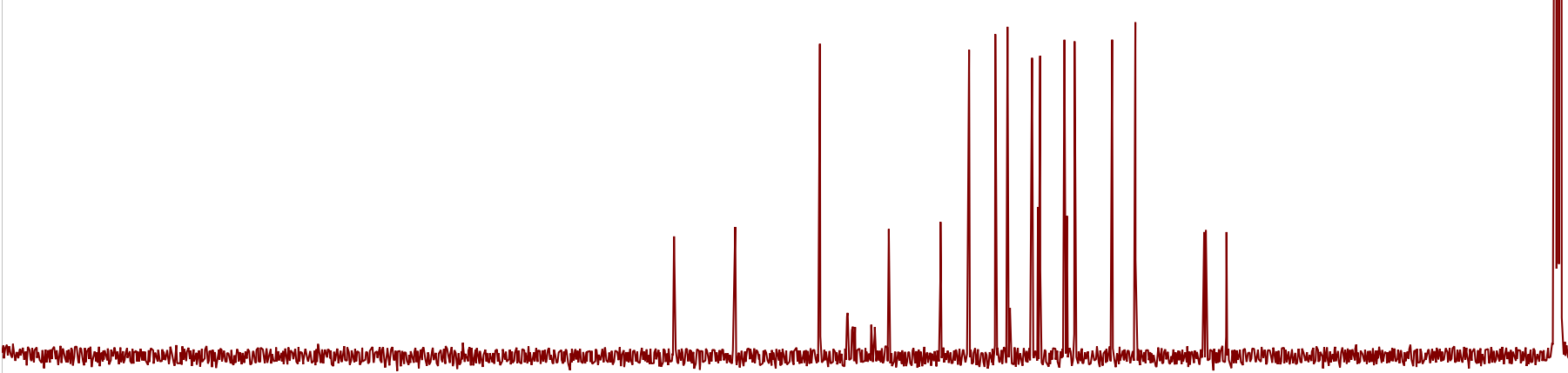

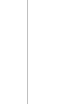

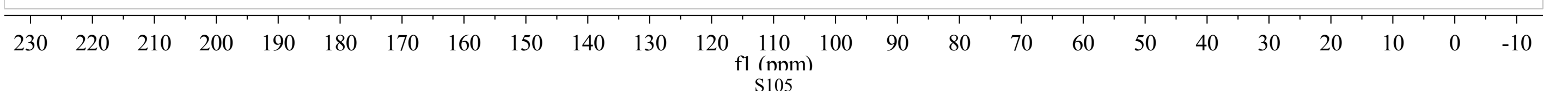




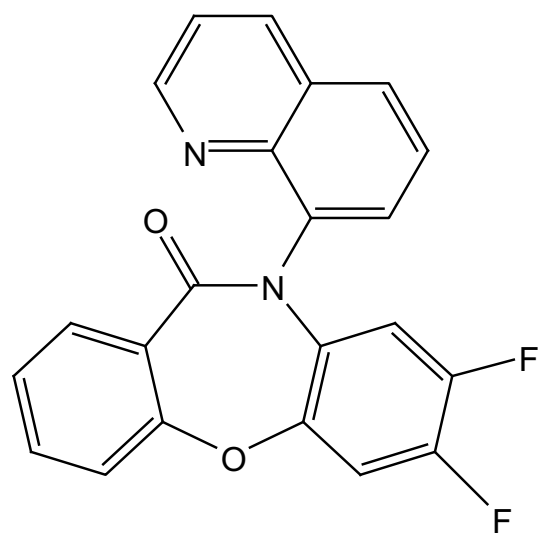

$7 \mathrm{t}$

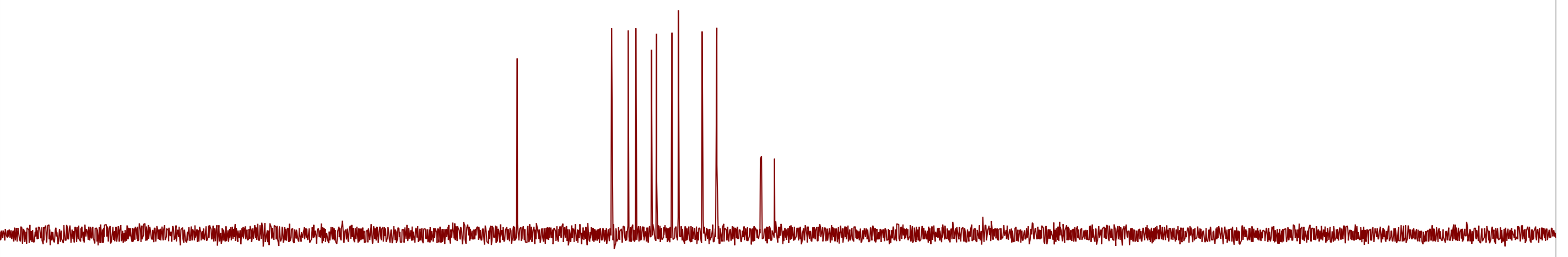

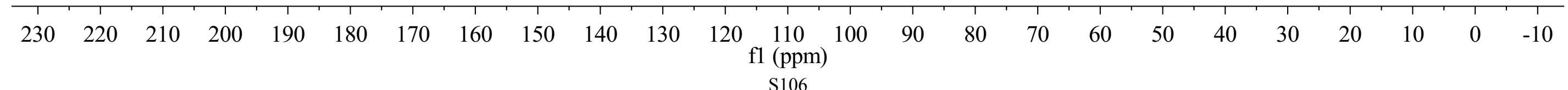



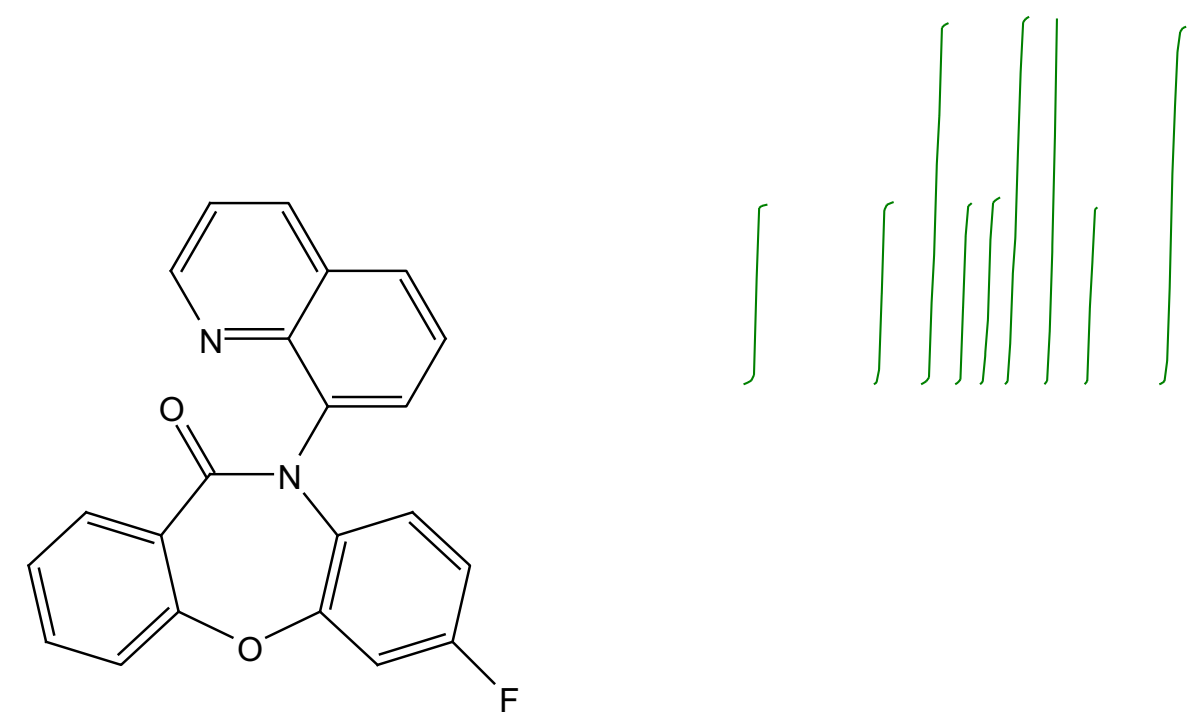

$7 u$

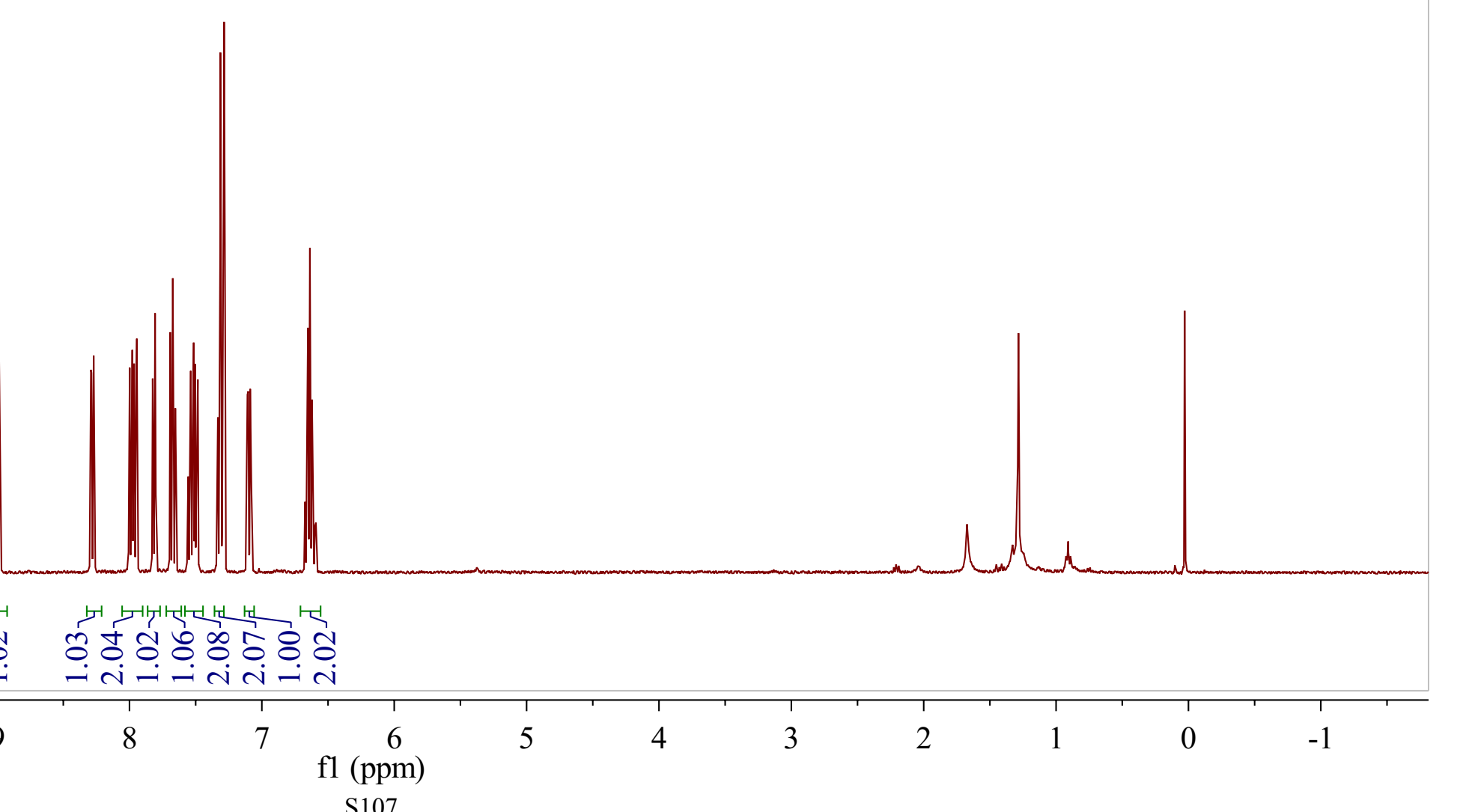



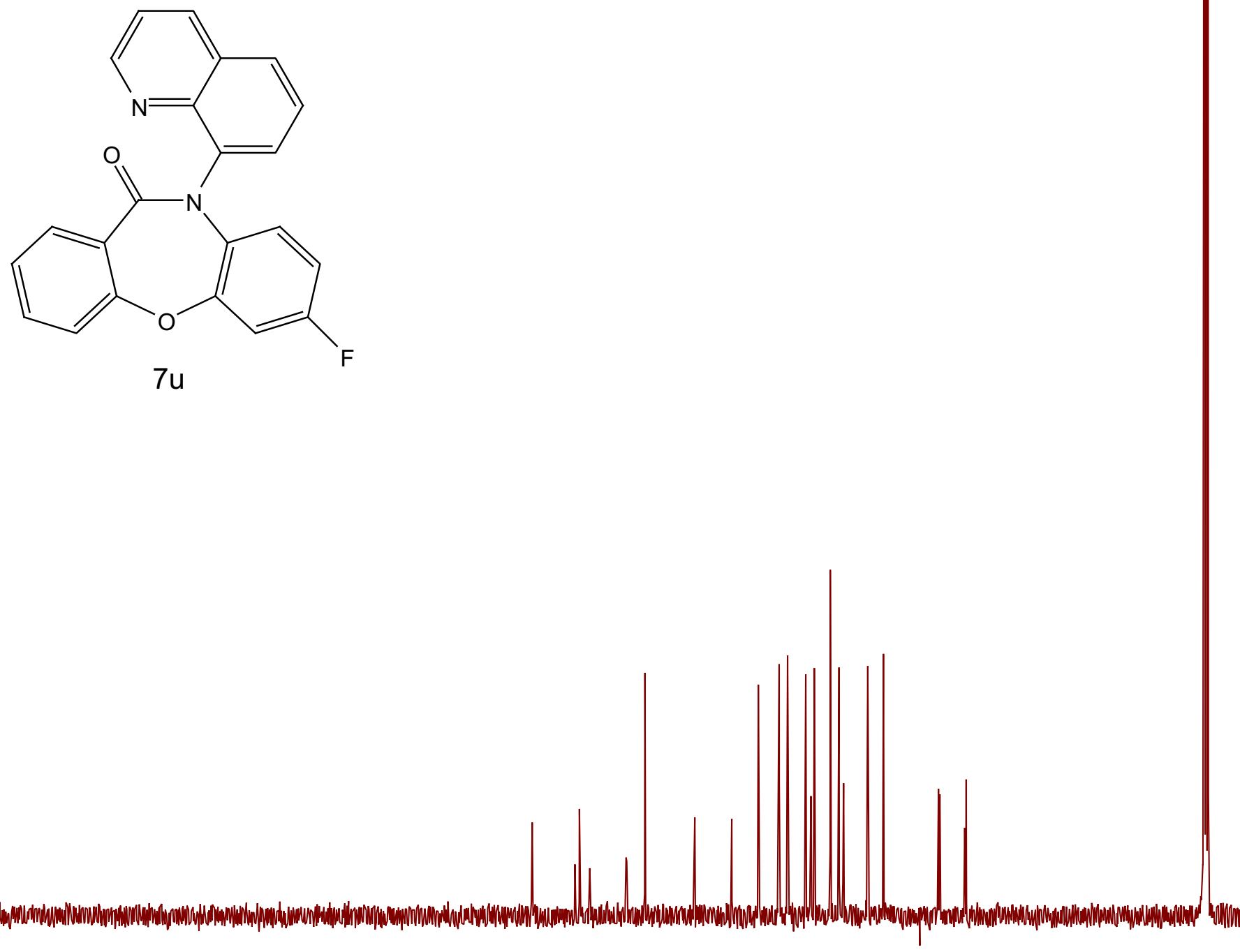

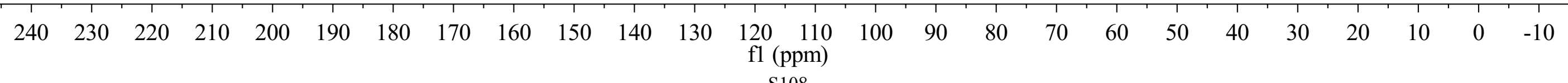



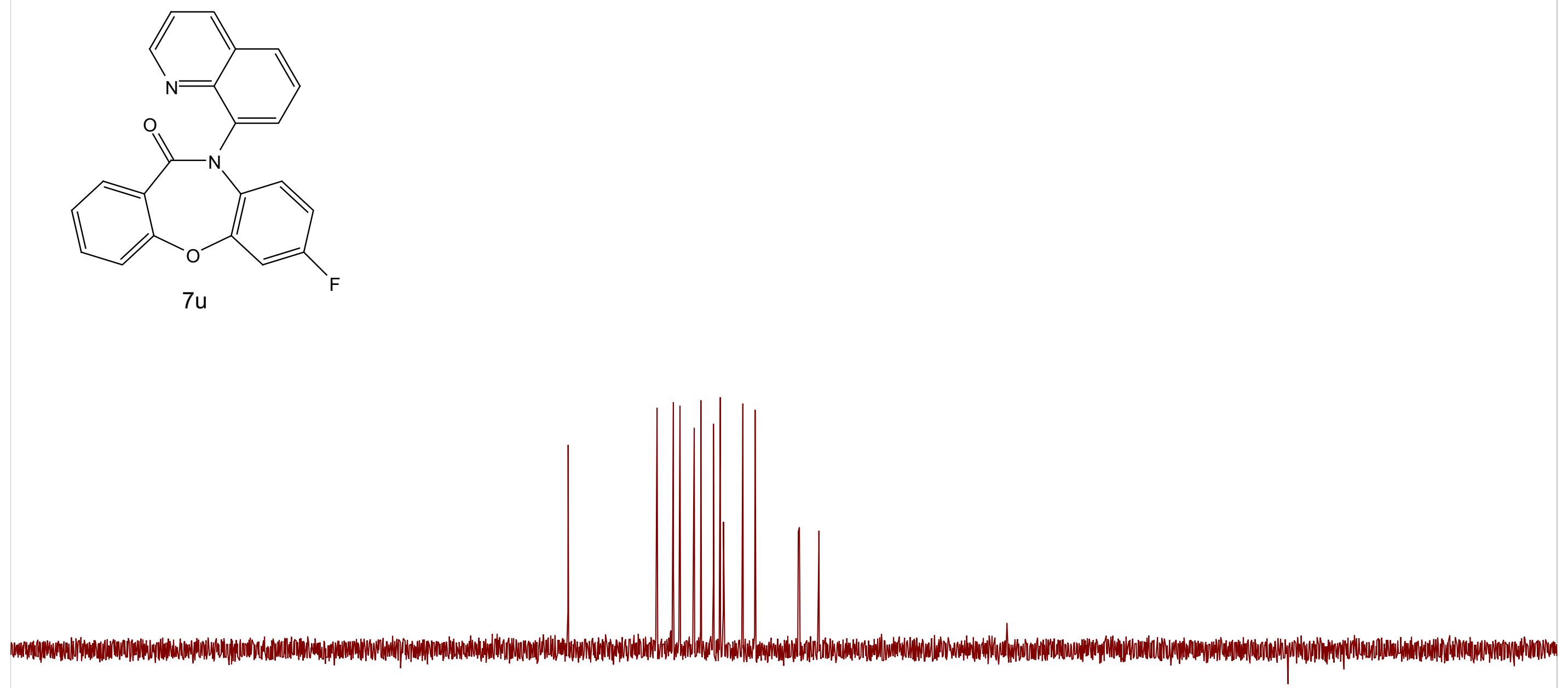

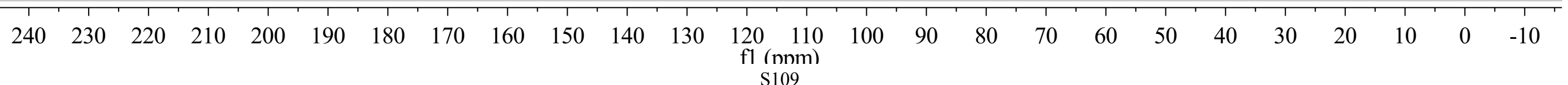



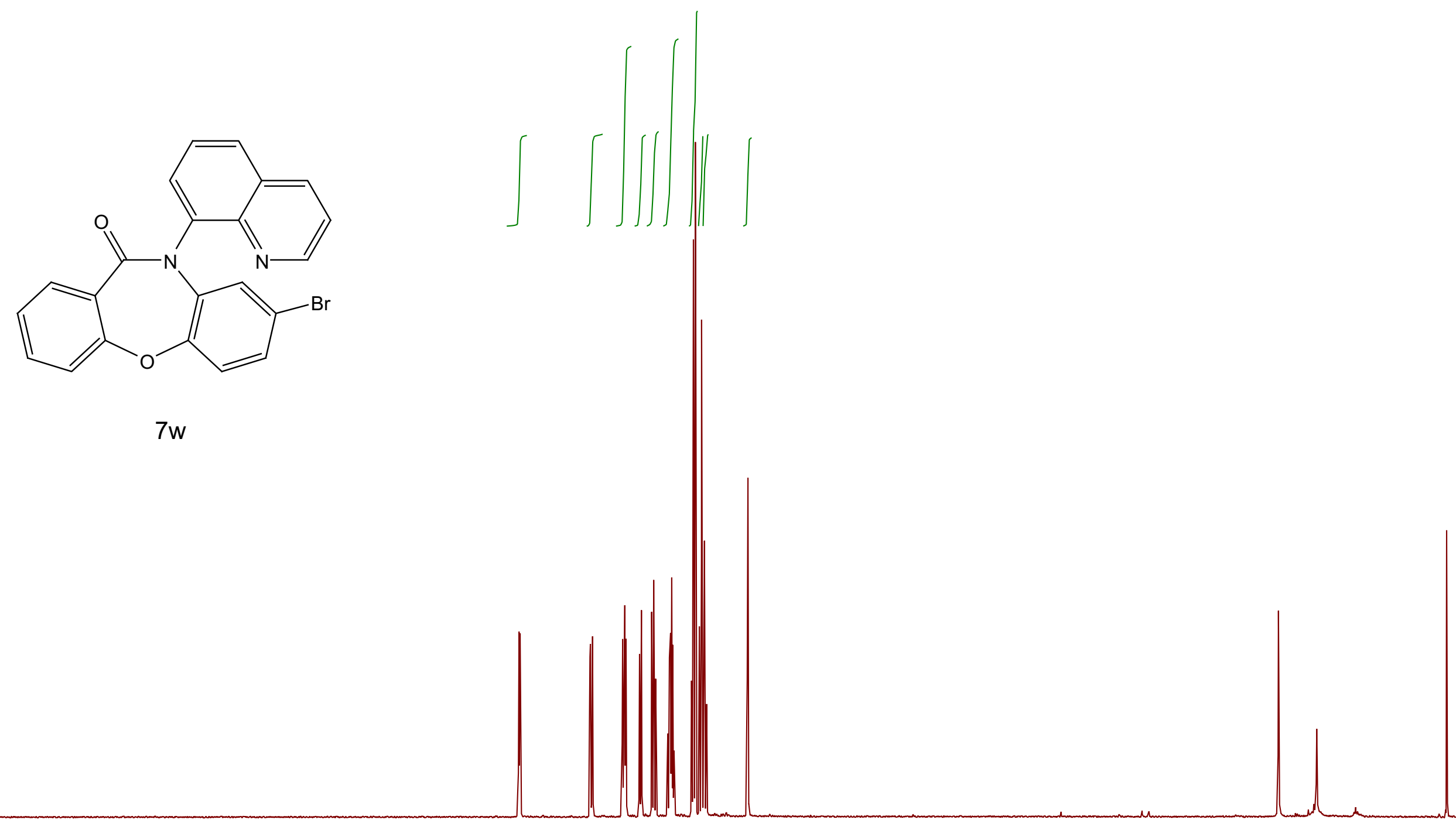

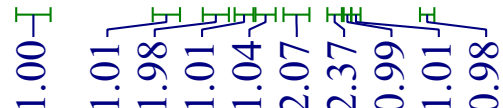

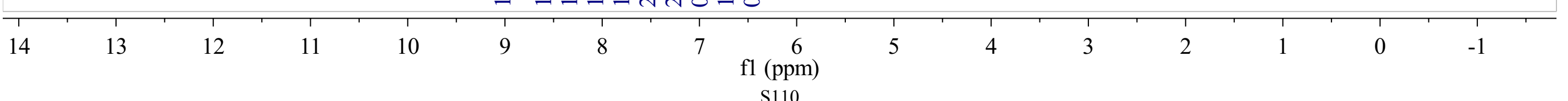




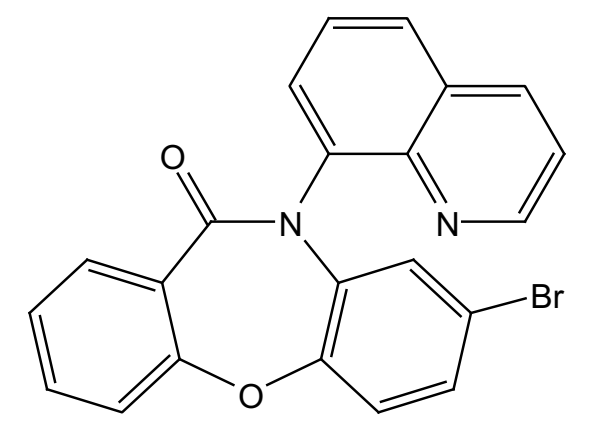

$7 w$

\begin{tabular}{lllllllllllllllllllllllll}
\hline 230 & 220 & 210 & 200 & 190 & 180 & 170 & 160 & 150 & 140 & 130 & 120 & 110 & 100 & 90 & 80 & 70 & 60 & 50 & 40 & 30 & 20 & 10 & 0 & -10
\end{tabular}




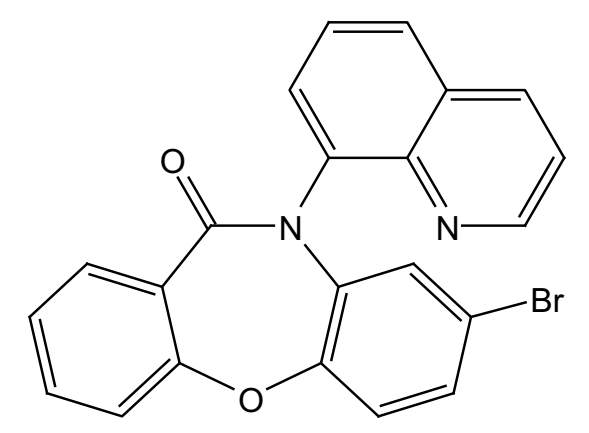

$7 w$

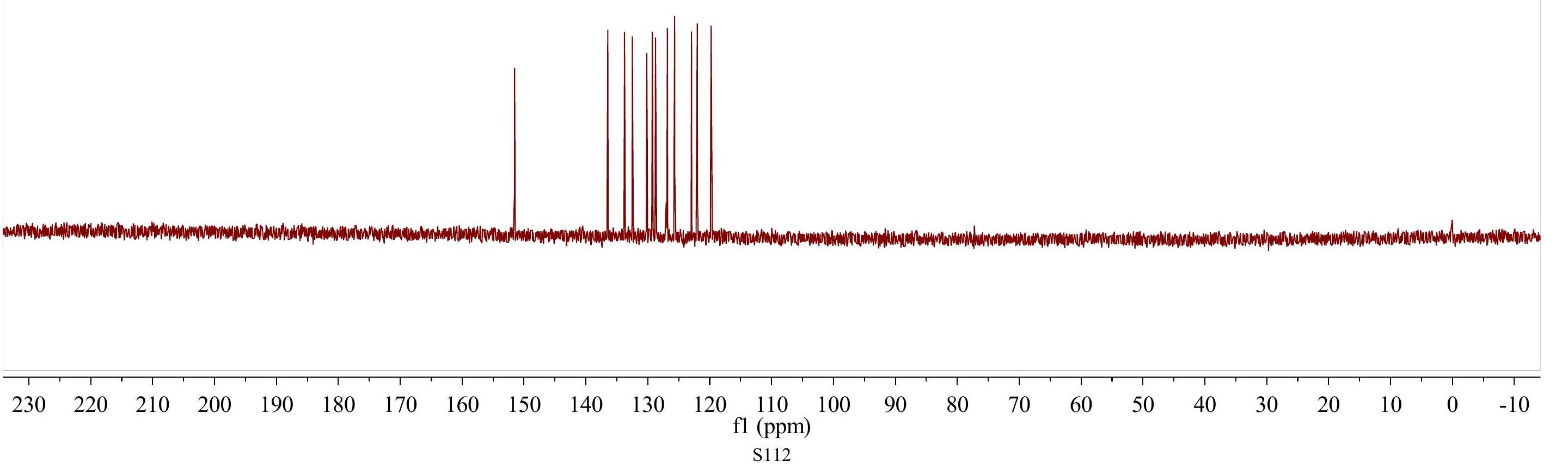



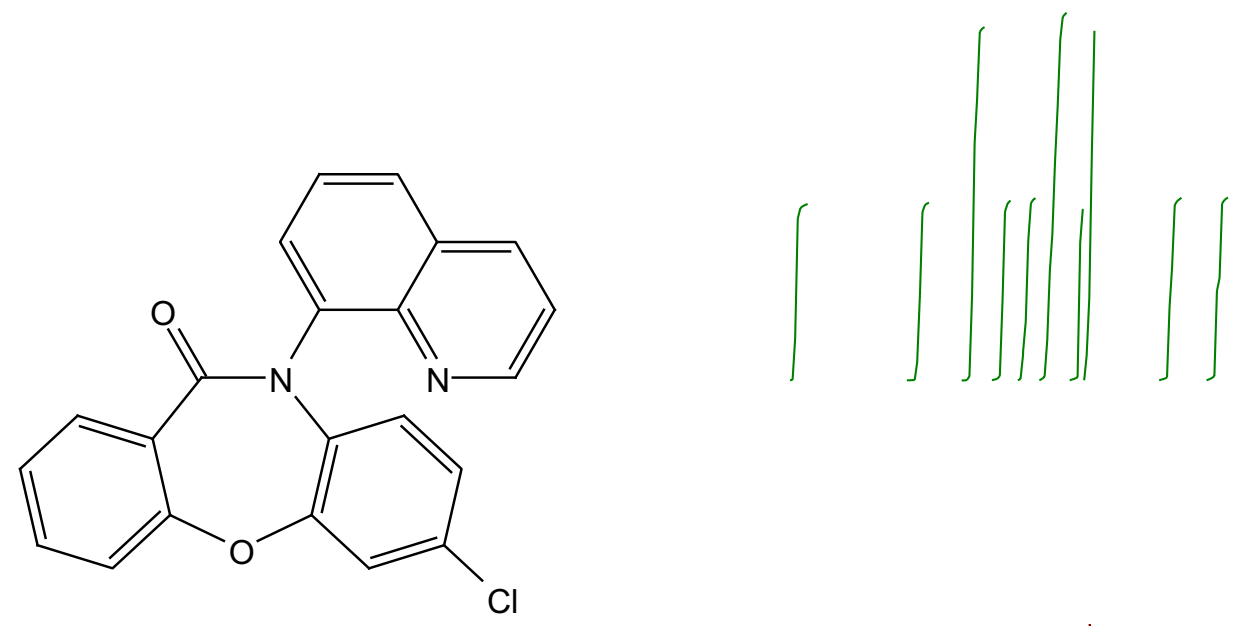

$8 a$

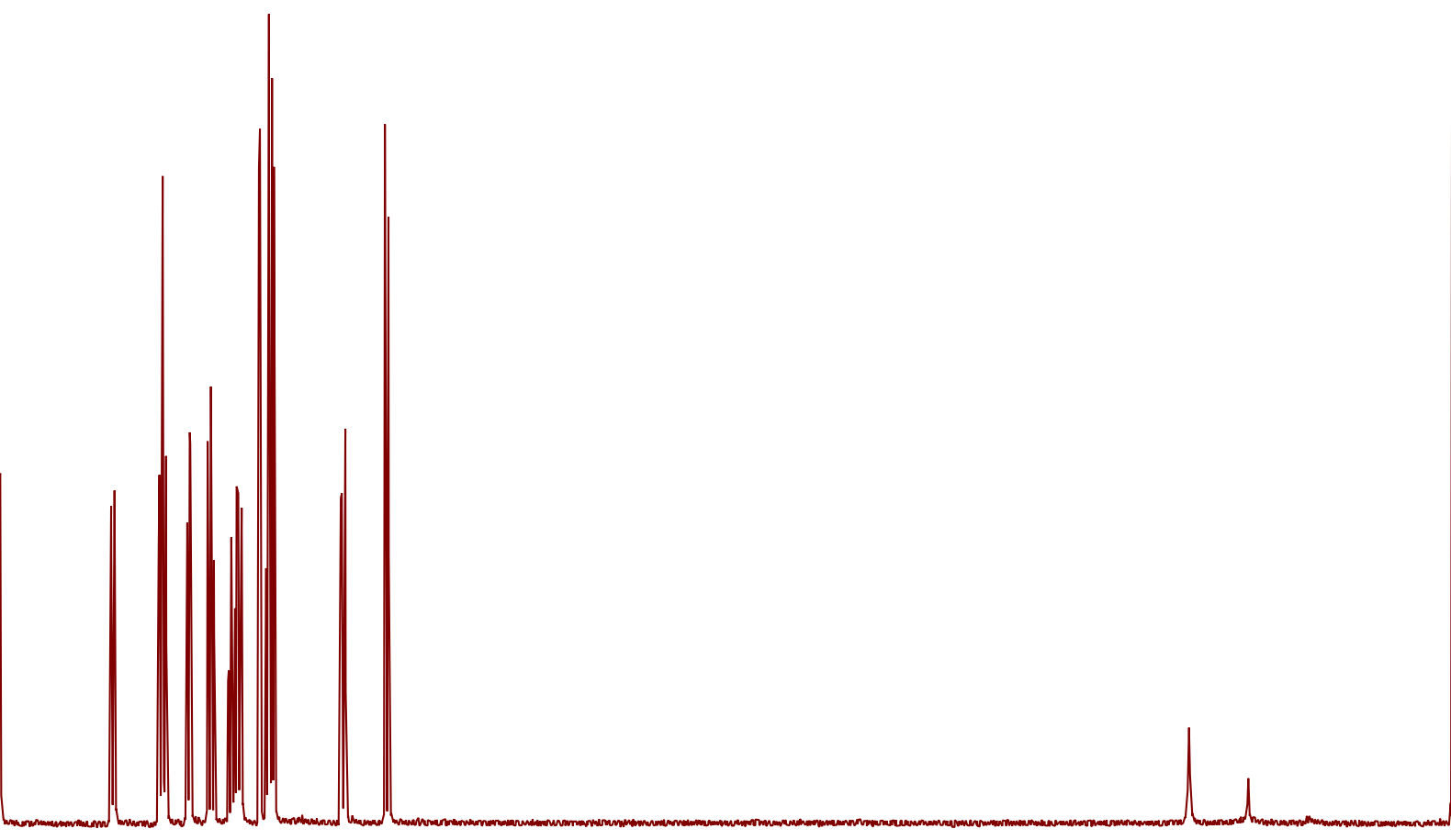

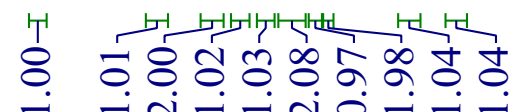

12

11

10

9

8

7 fl (ppm) 


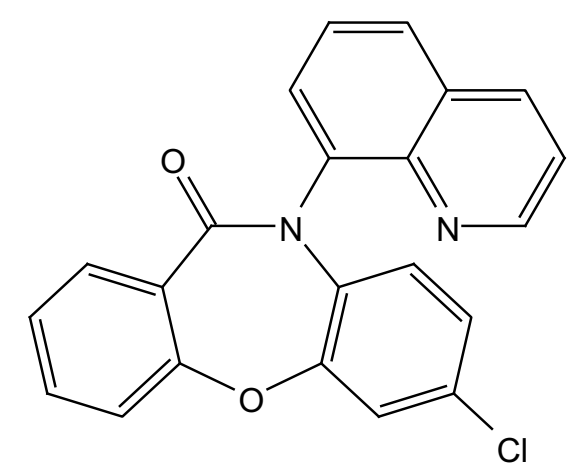

$8 a$

$\begin{array}{lllllllllllllllllllllllllllll} & 40 & 230 & 220 & 210 & 200 & 190 & 180 & 170 & 160 & 150 & 140 & 130 & 120 & 110 & 100 & 90 & 80 & 70 & 60 & 50 & 40 & 30 & 20 & 10 & 0 & -10 & -2\end{array}$




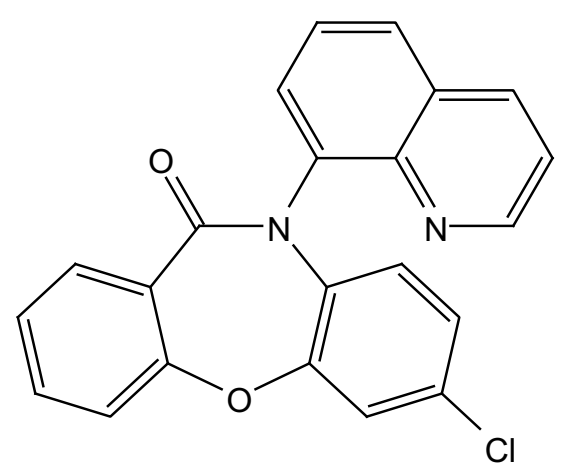

$8 a$ 

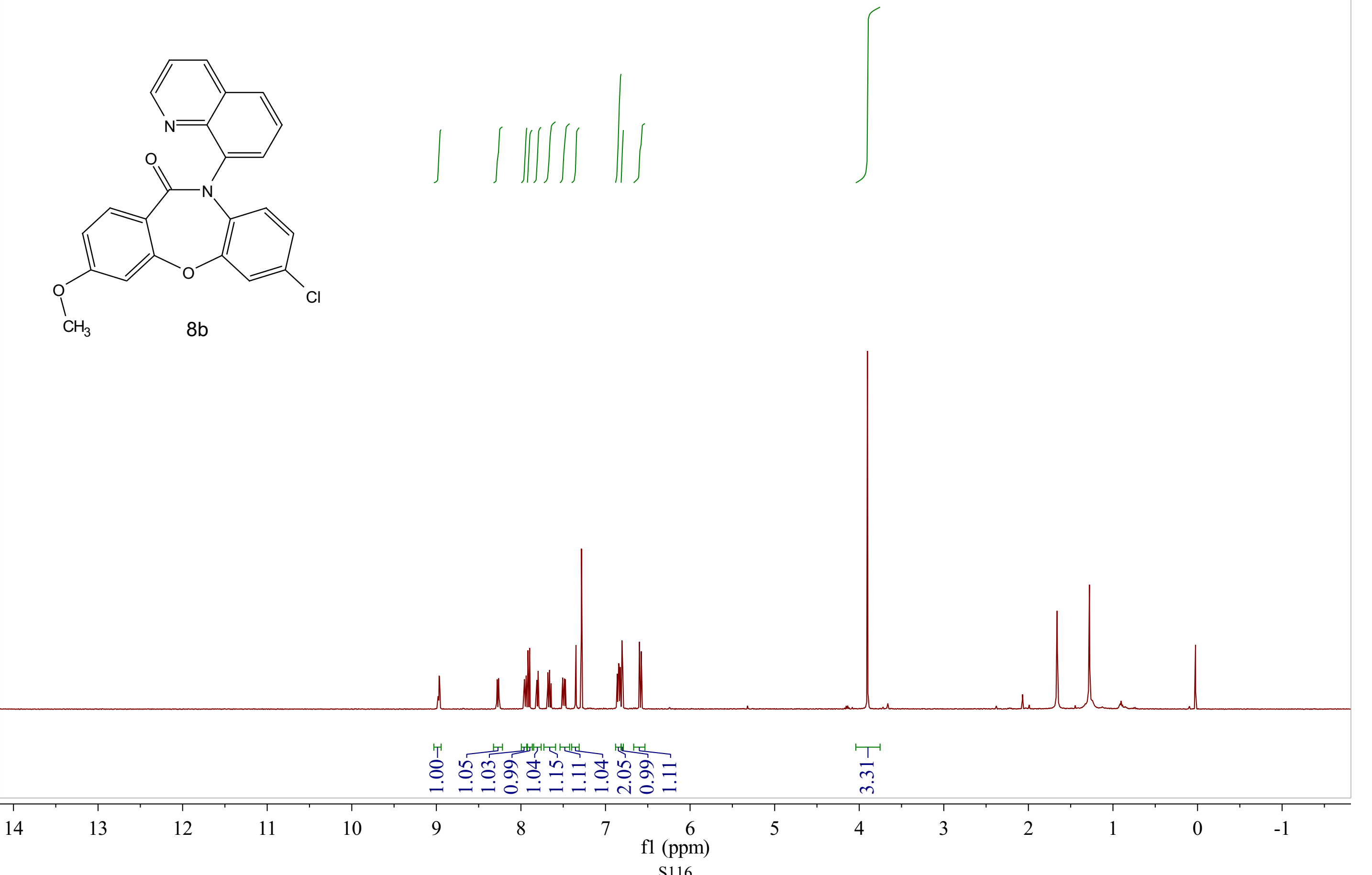


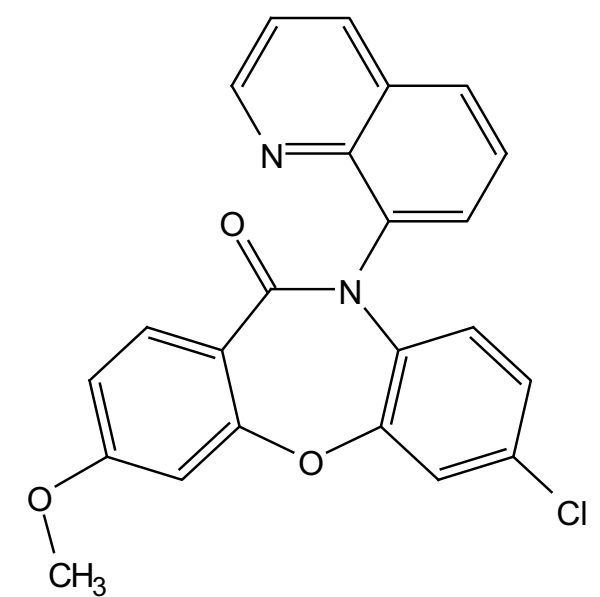

$8 b$

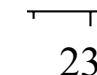




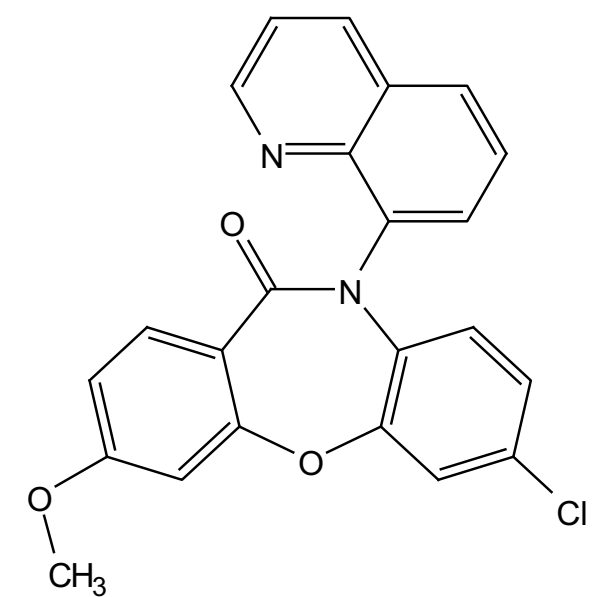

$8 b$

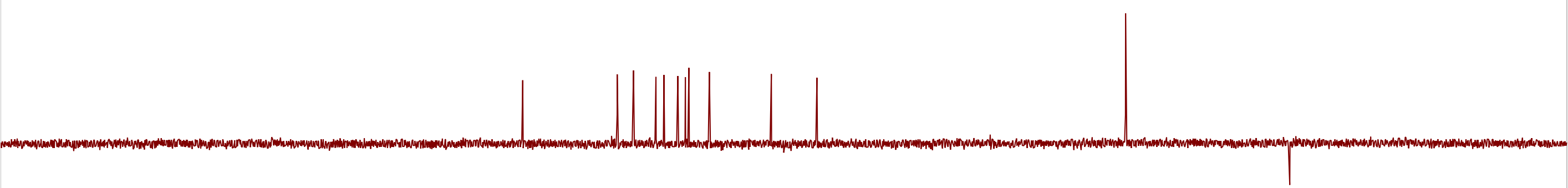

$\begin{array}{lllllllllllllllllllllllll}230 & 220 & 210 & 200 & 190 & 180 & 170 & 160 & 150 & 140 & 130 & 120 & \begin{array}{c}110 \\ \mathrm{fl}(\mathrm{ppm})\end{array} & 100 & 90 & 80 & 70 & 60 & 50 & 40 & 30 & 20 & 10 & 0 & -10\end{array}$




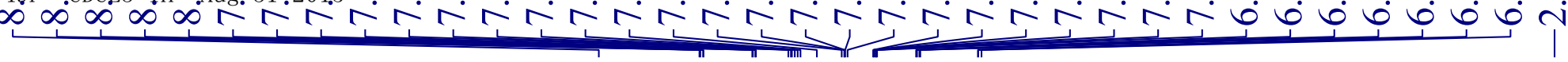
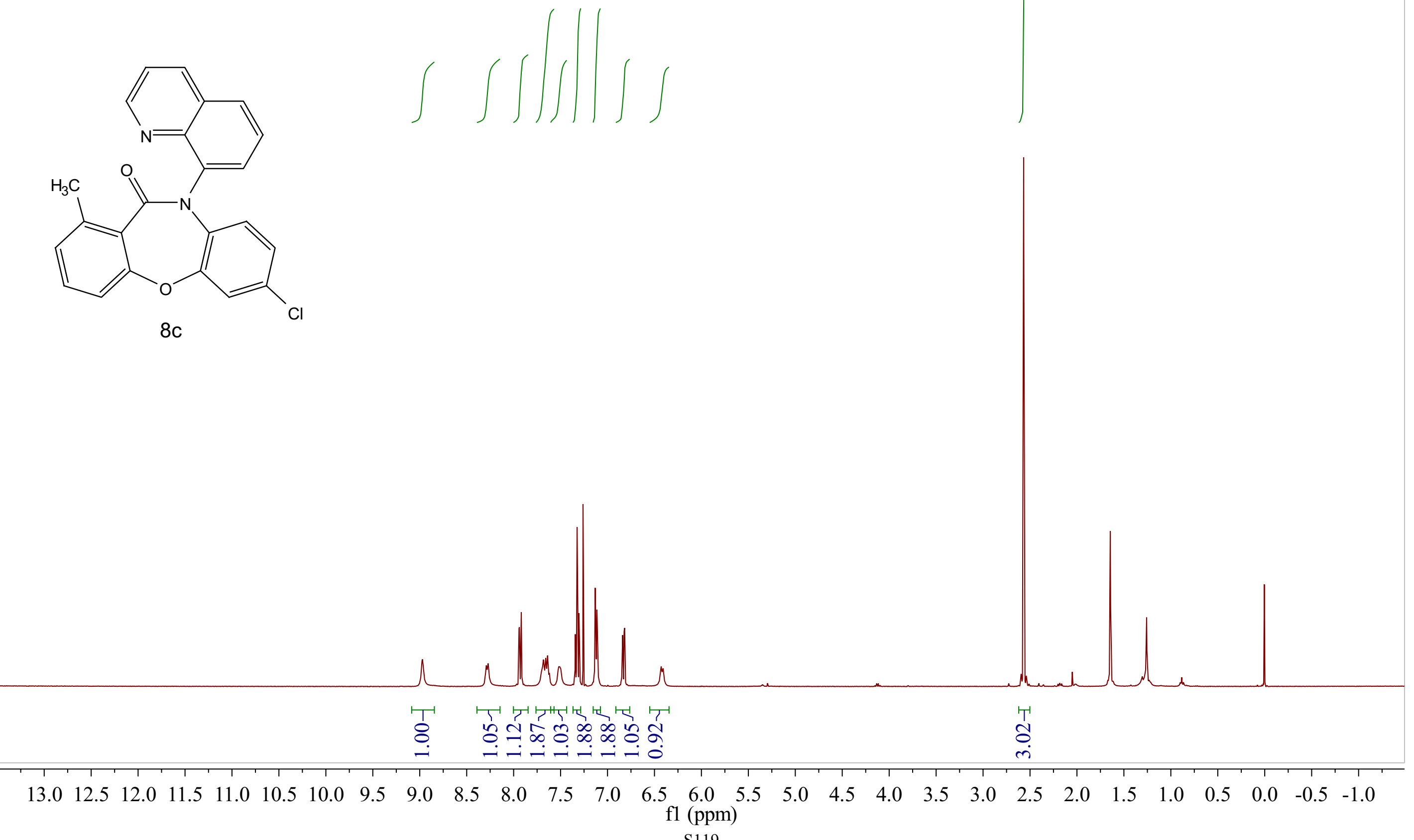


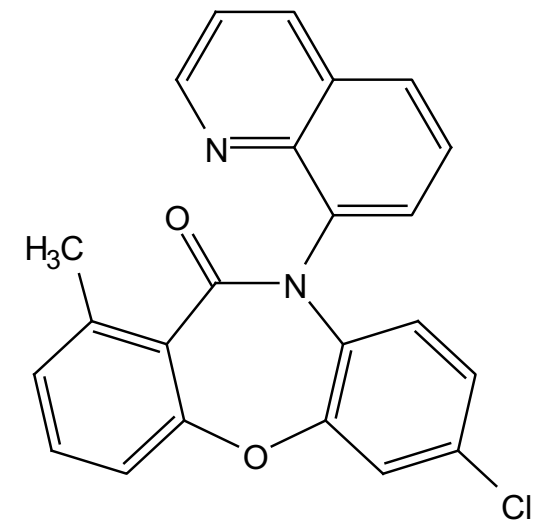

$8 c$

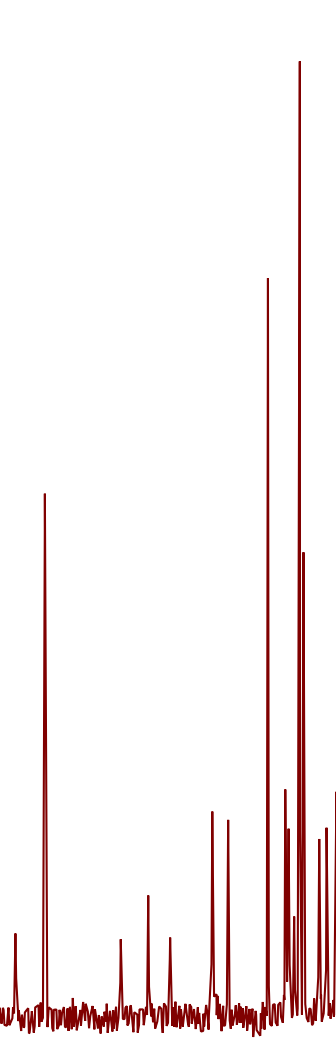




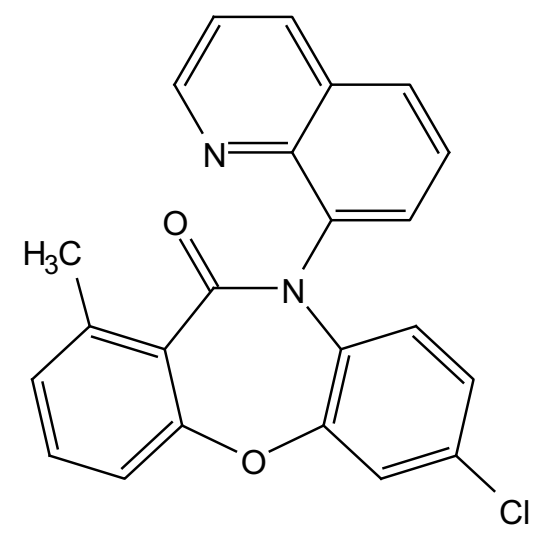

$8 c$

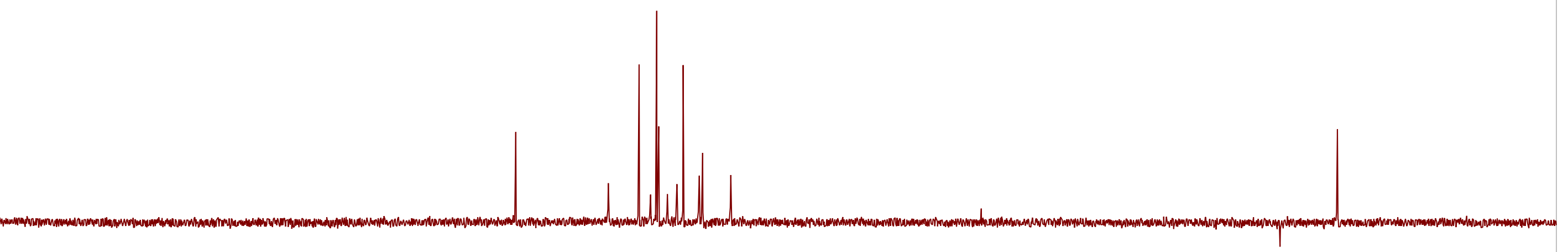

\begin{tabular}{lllllllllllllllllllllllllllll}
\hline & 230 & 220 & 210 & 200 & 190 & 180 & 170 & 160 & 150 & 140 & 130 & 120 & 110 & 100 & 90 & 80 & 70 & 60 & 50 & 40 & 30 & 20 & 10 & 0 & -10
\end{tabular}




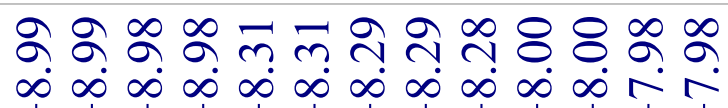
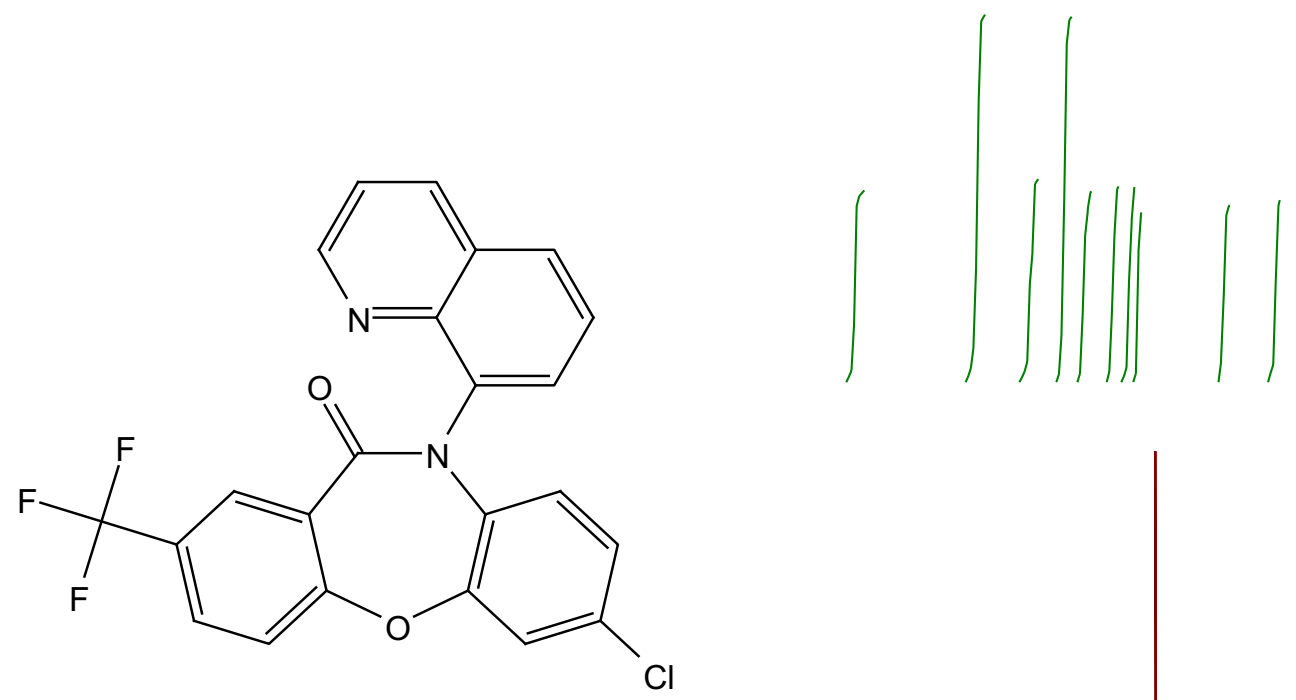

$8 d$

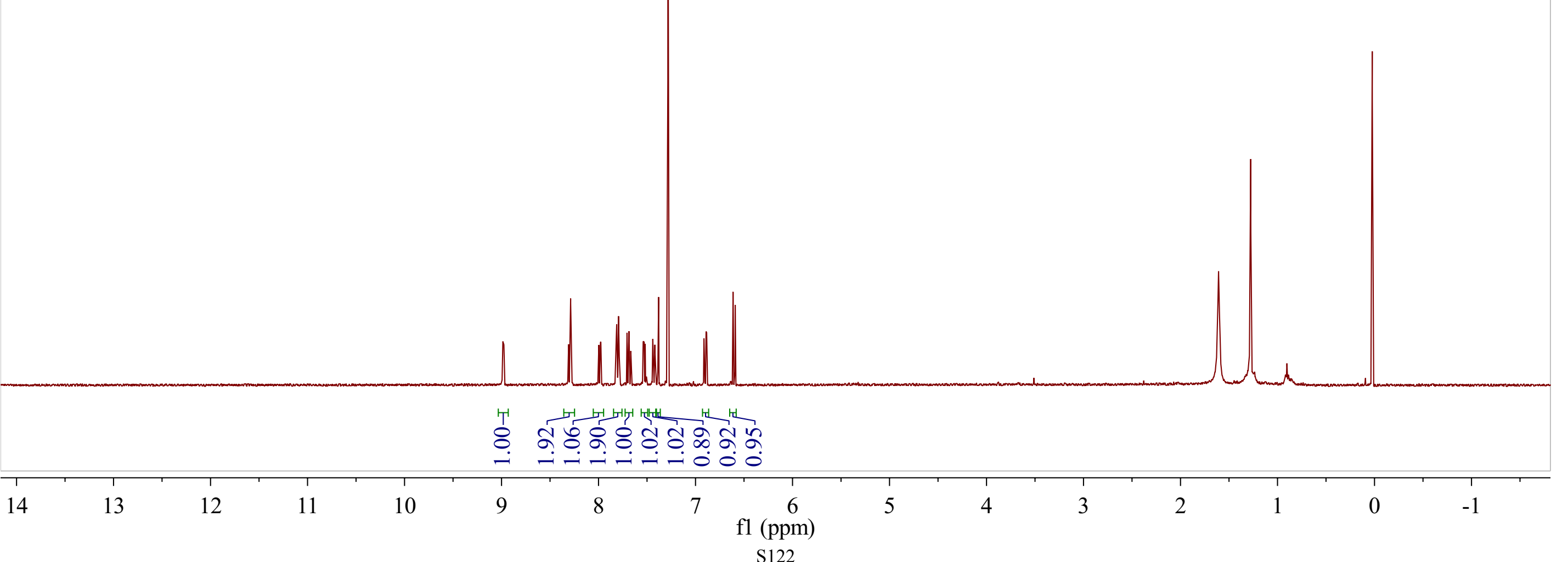




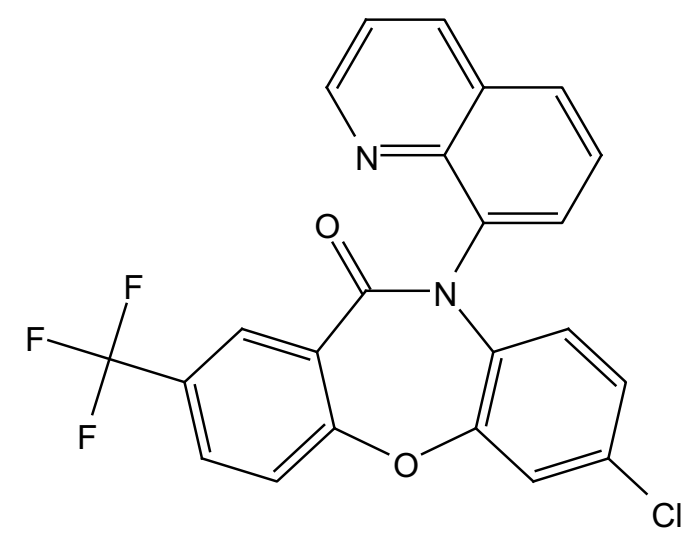

$8 d$
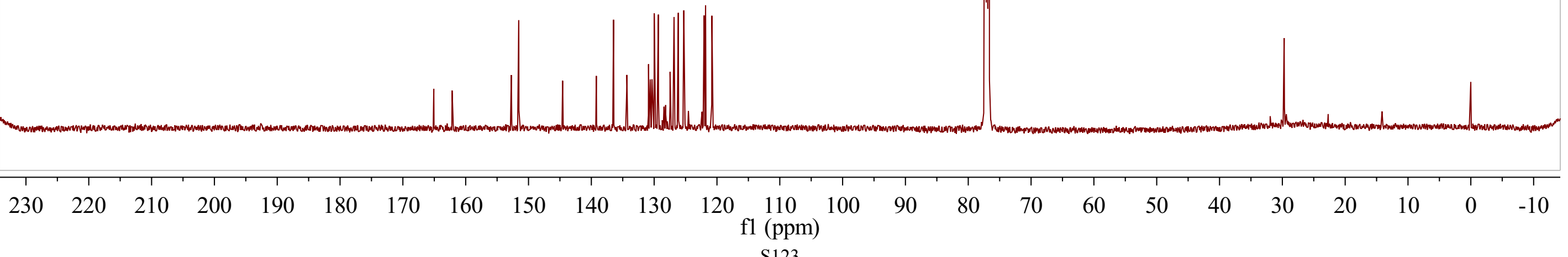


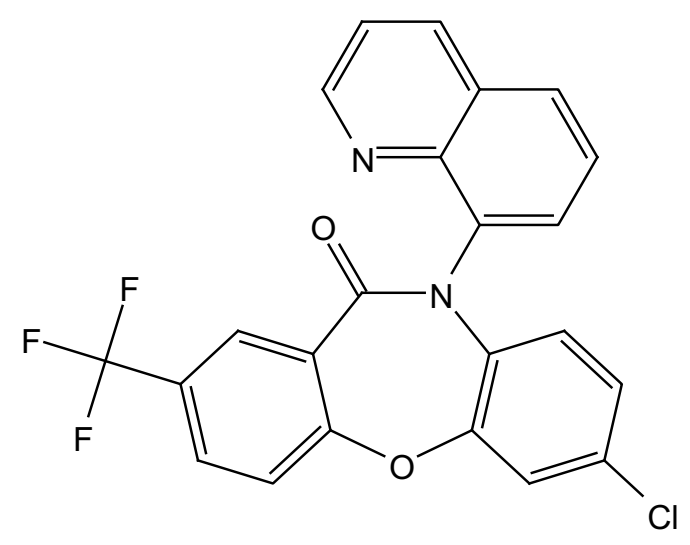

$8 d$

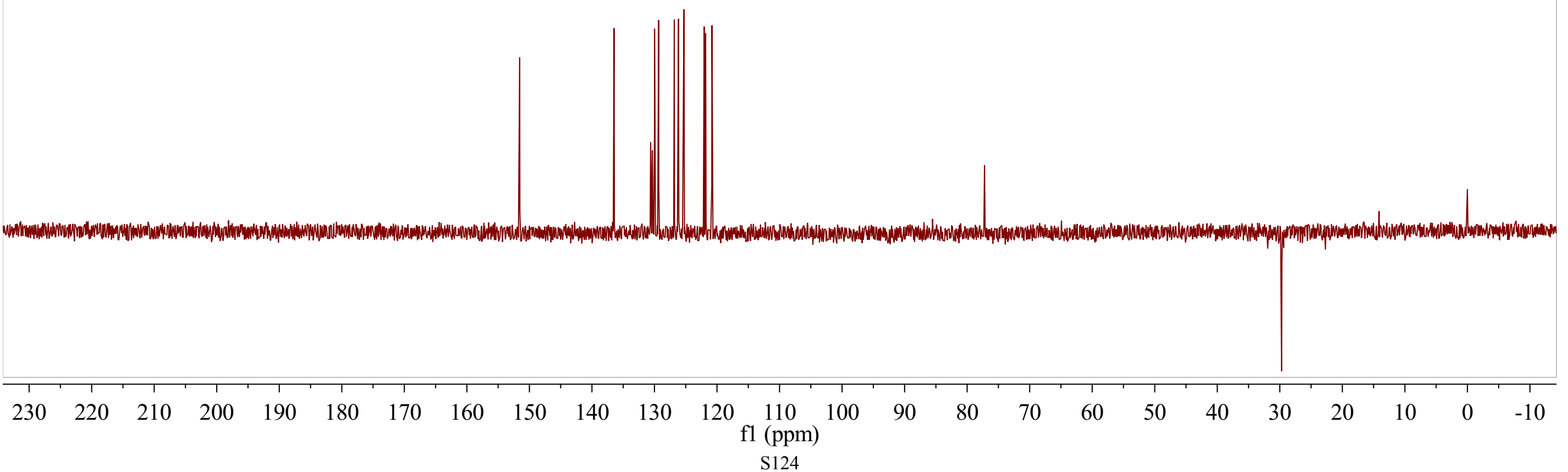



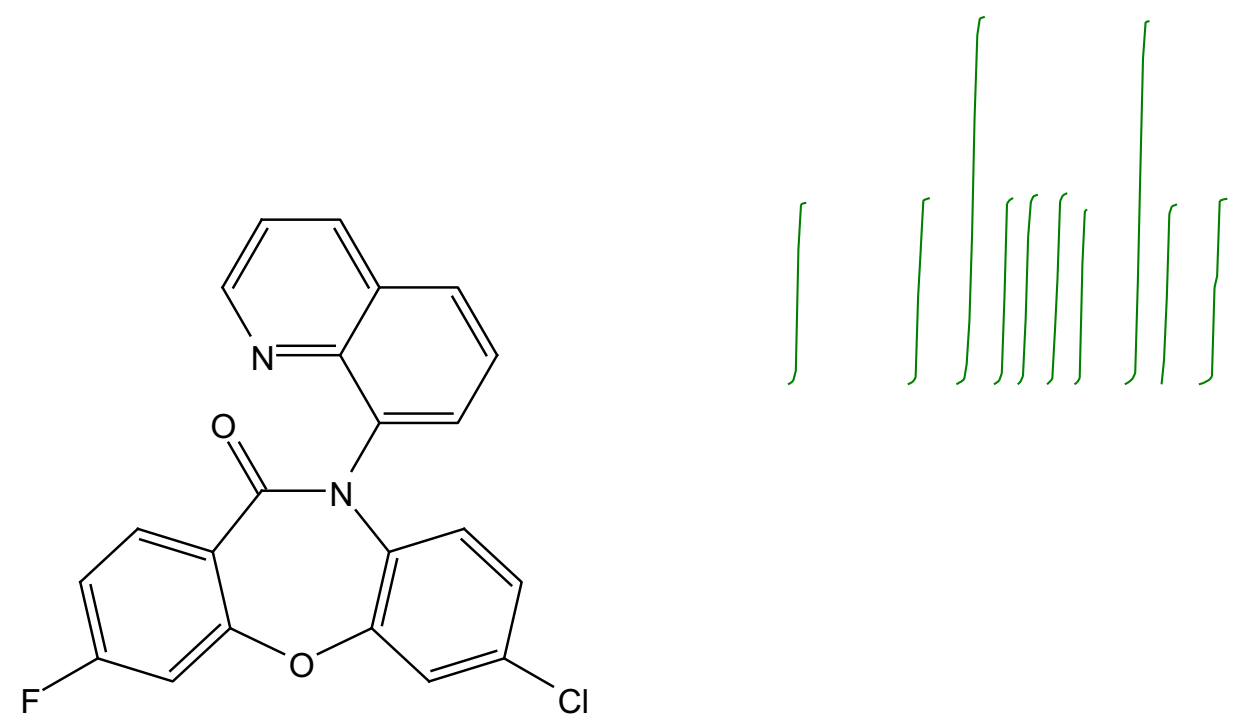

$8 e$

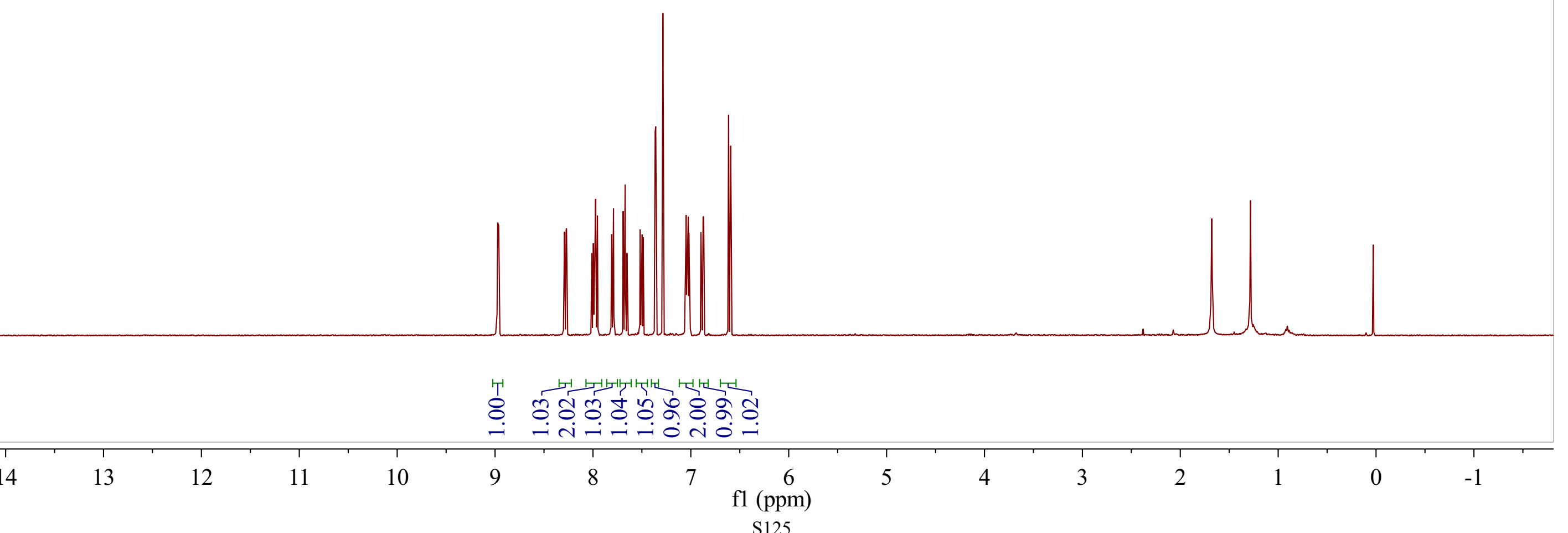




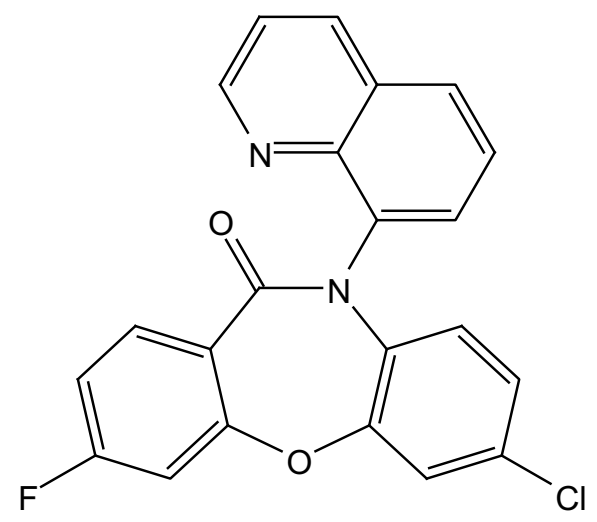

$8 e$ 


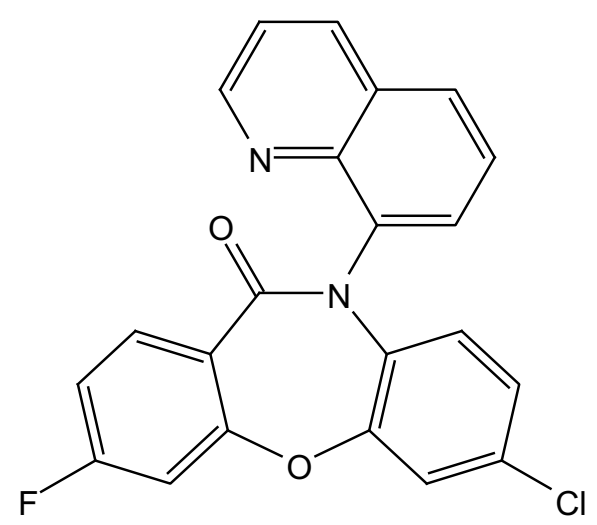

$8 e$

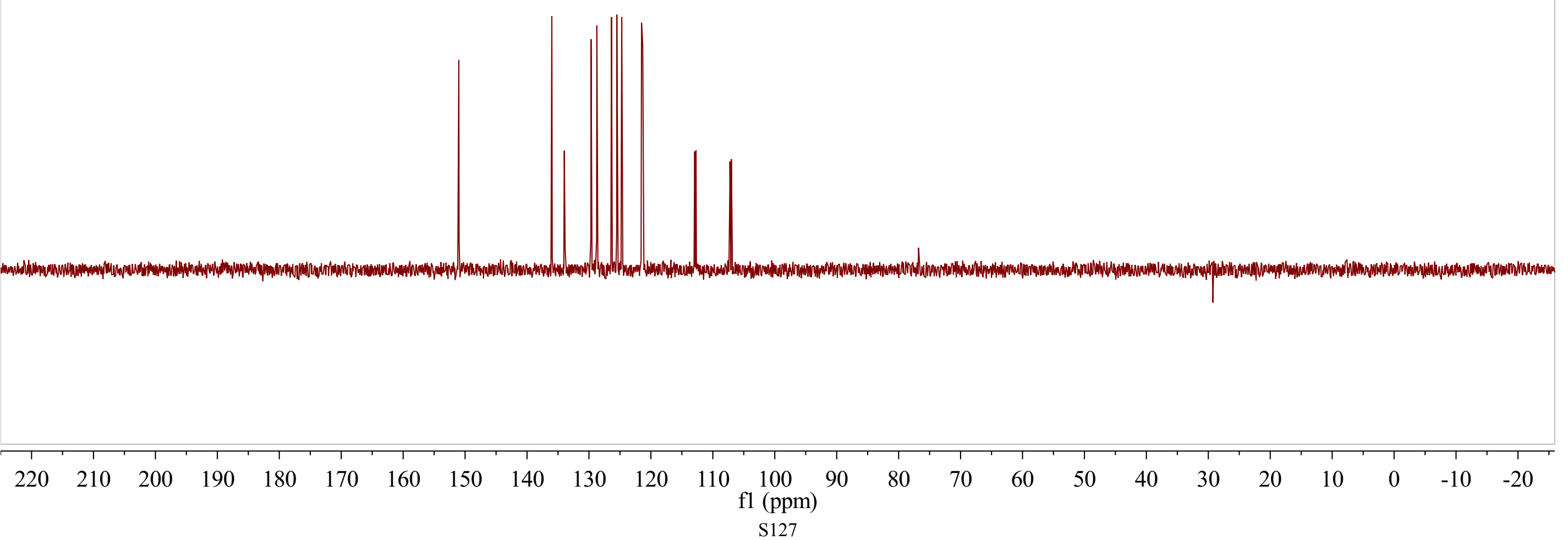



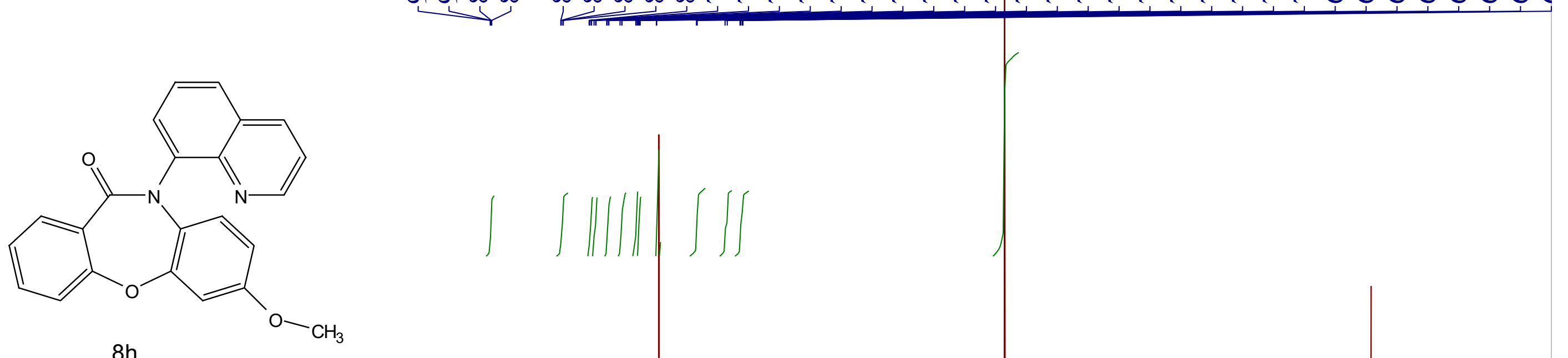

$8 h$

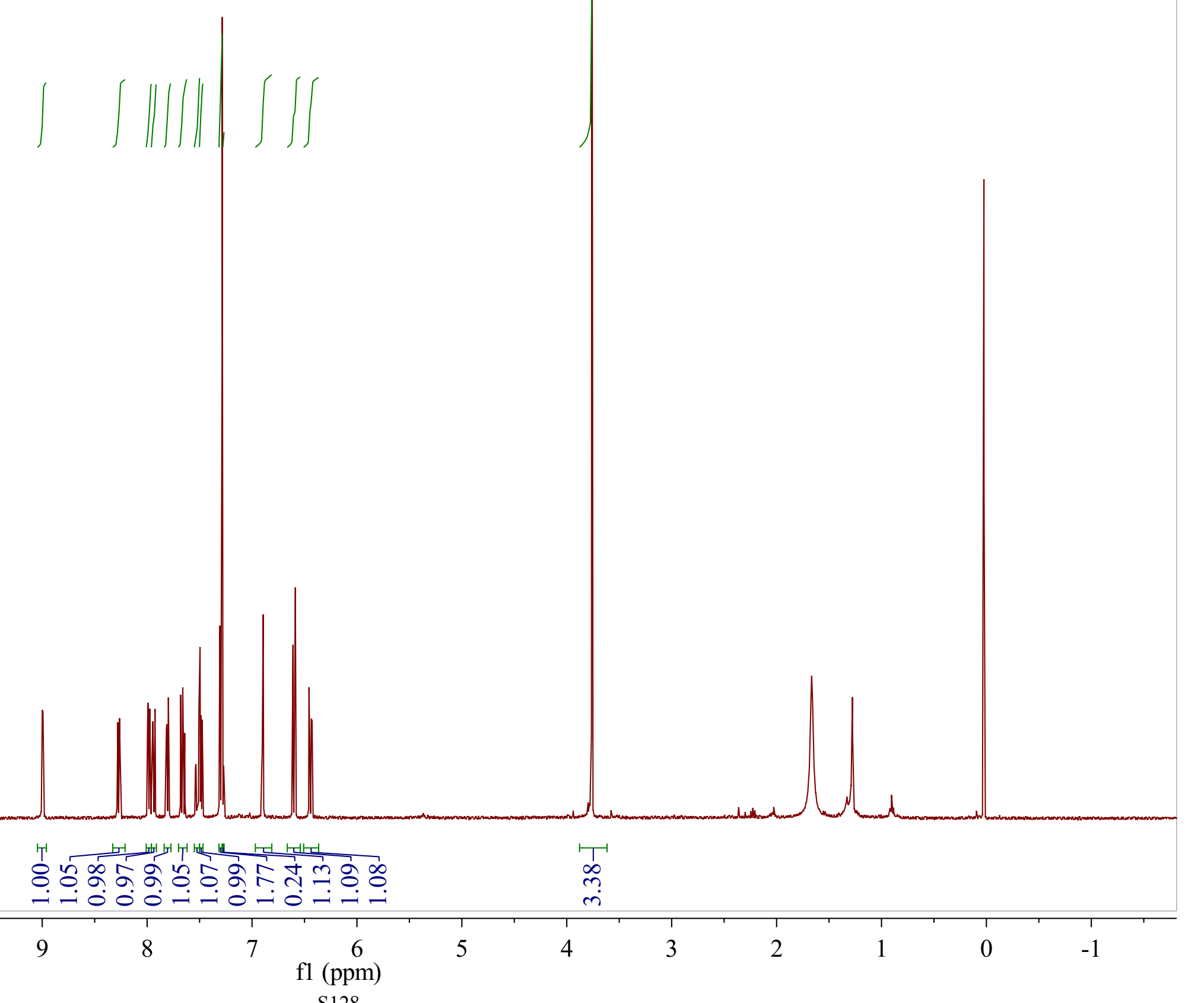


(1)

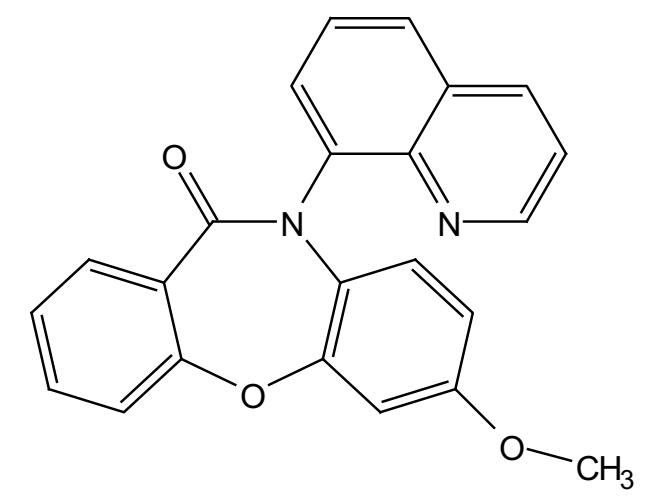

$8 \mathrm{~h}$

$$
230
$$




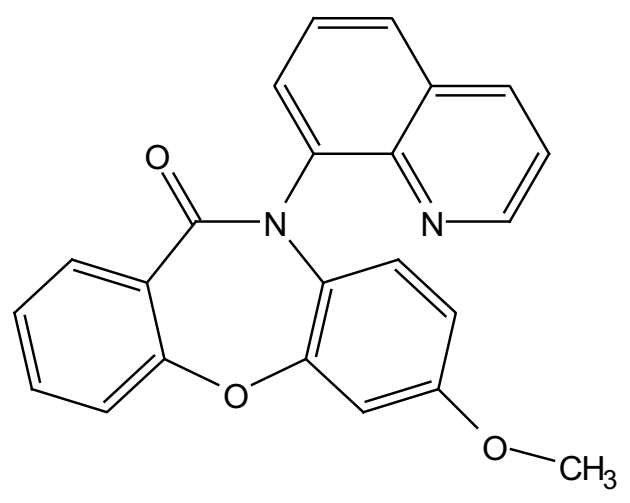

$8 h$

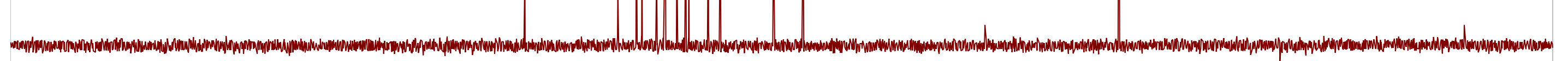

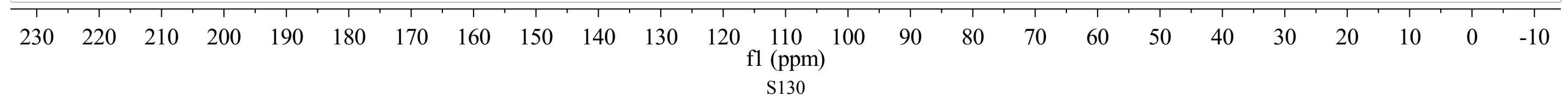



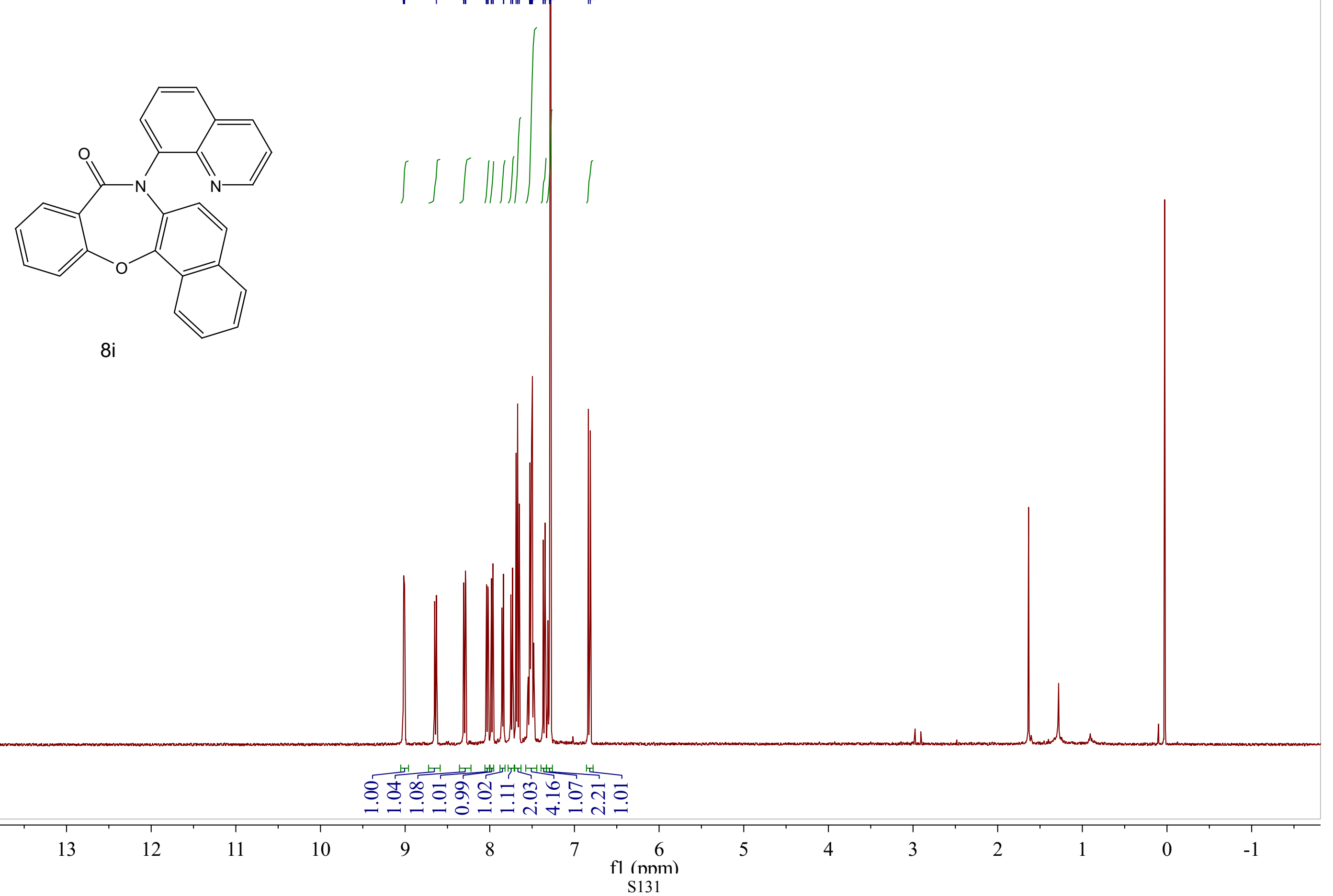


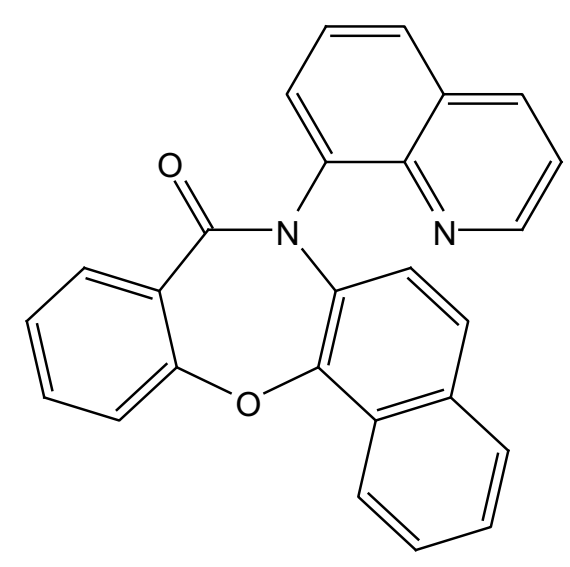

$8 \mathbf{i}$

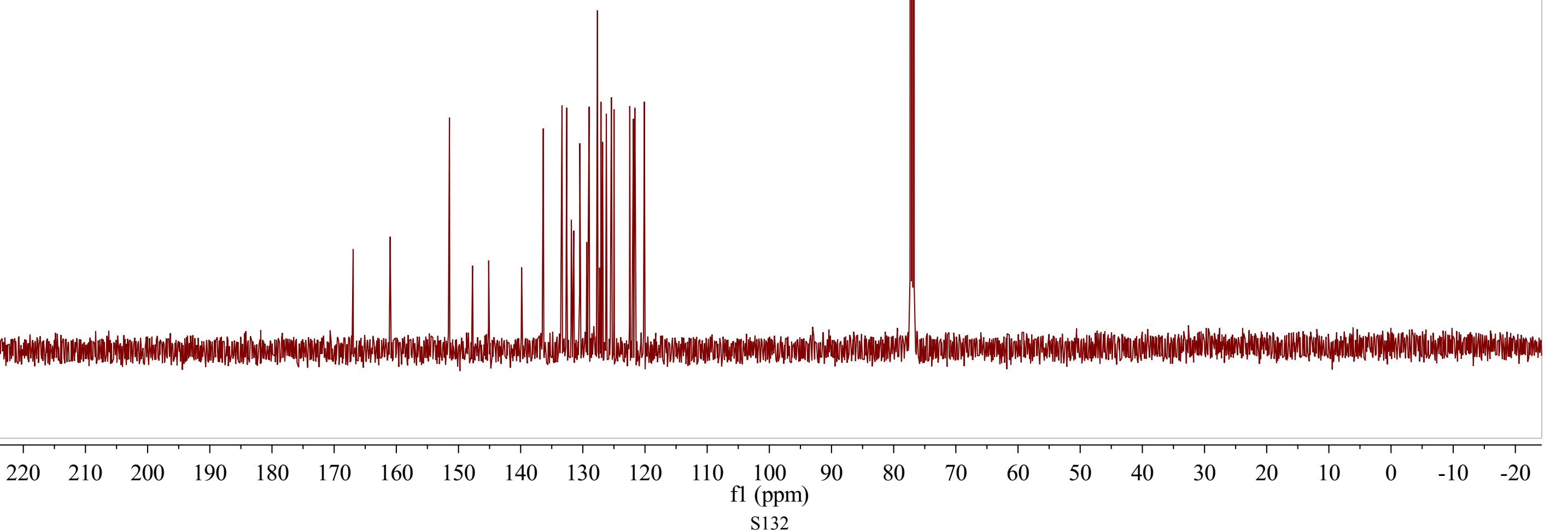




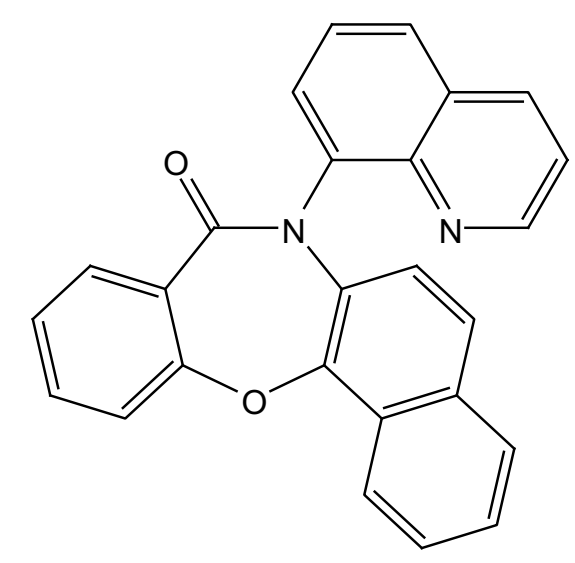

$8 \mathbf{i}$

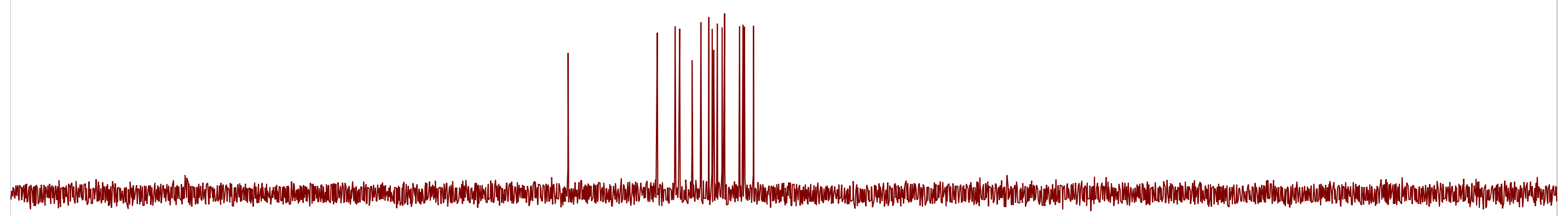

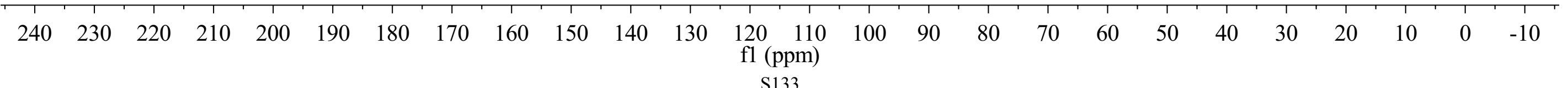




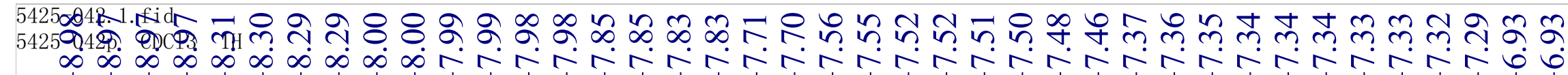
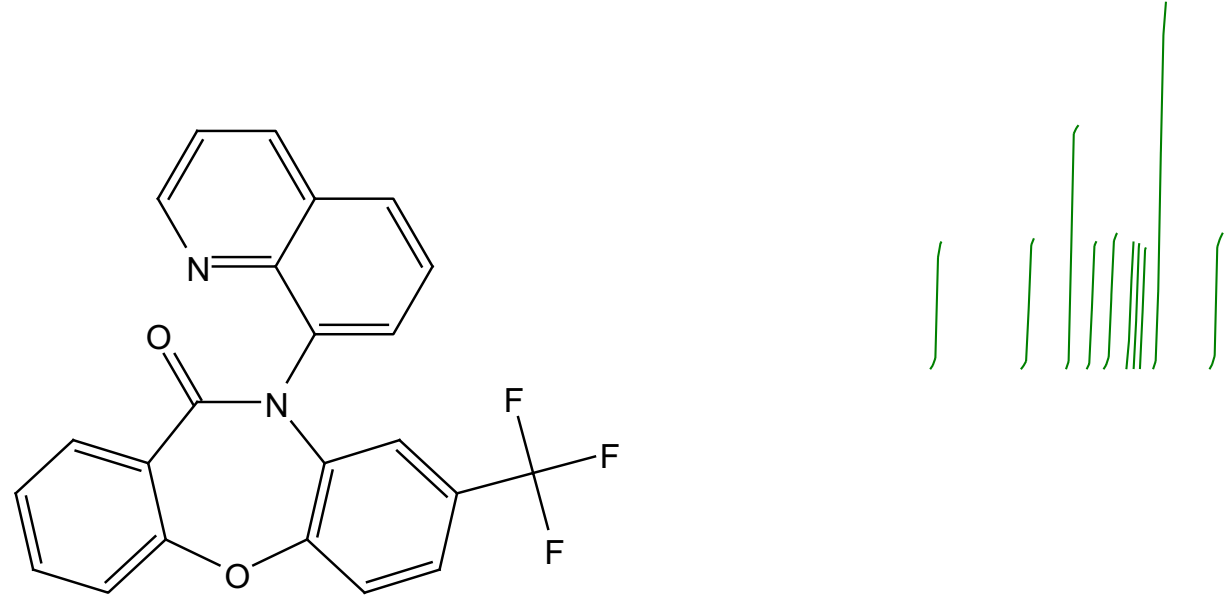

8j

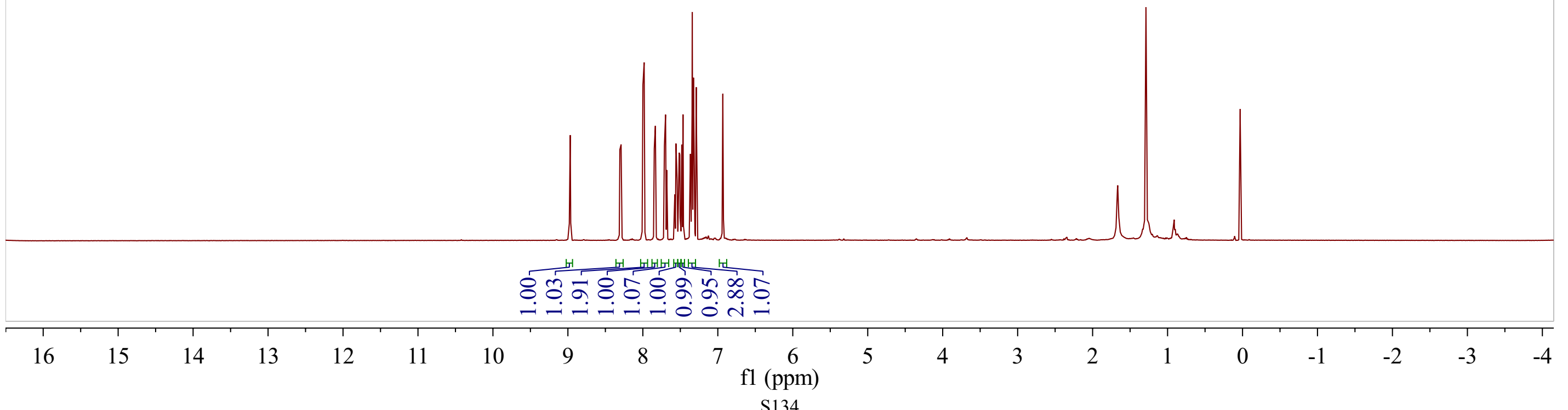




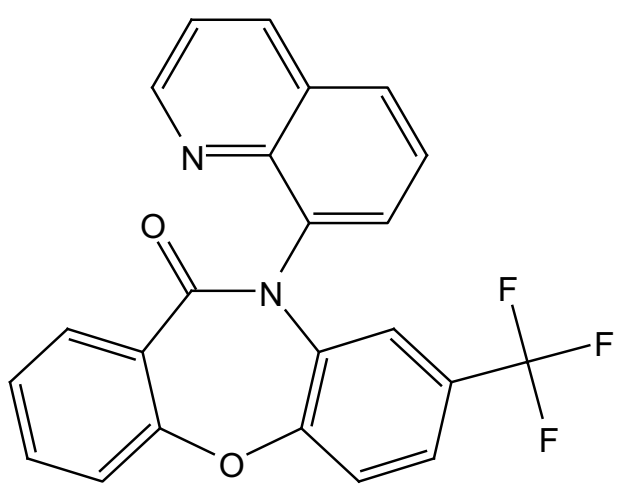

8j

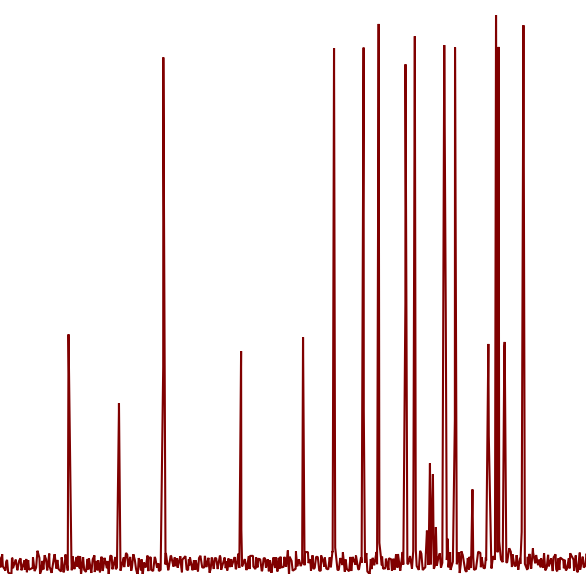

\begin{tabular}{llllllllllllllllllllllllll}
\hline 230 & 220 & 210 & 200 & 190 & 180 & 170 & 160 & 150 & 140 & 130 & 120 & $\begin{array}{c}110 \\
\mathrm{fl}(\mathrm{ppm})\end{array}$ & 100 & 90 & 80 & 70 & 60 & 50 & 40 & 30 & 20 & 10 & 0 & -10
\end{tabular}




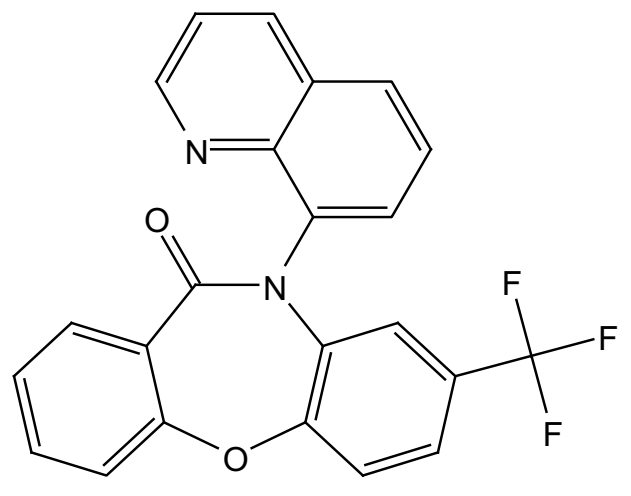

\begin{tabular}{lllllllllllllllllllllllll}
\hline 230 & 220 & 210 & 200 & 190 & 180 & 170 & 160 & 150 & 140 & 130 & 120 & 110 & 100 & 90 & 80 & 70 & 60 & 50 & 40 & 30 & 20 & 10 & 0 & -10
\end{tabular}



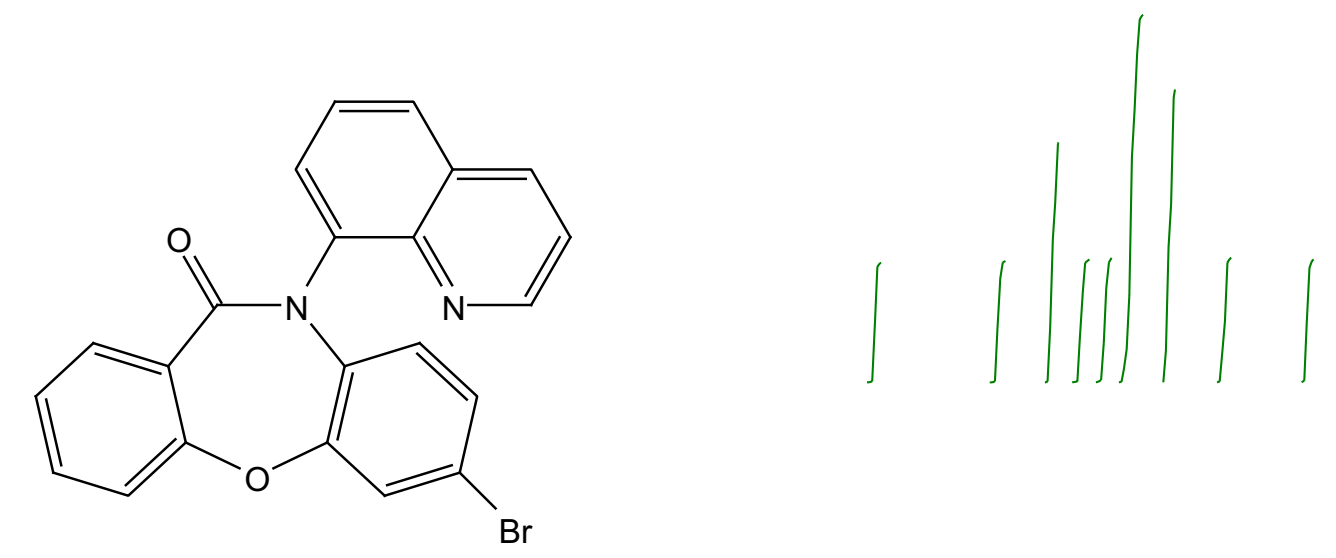

$8 k$

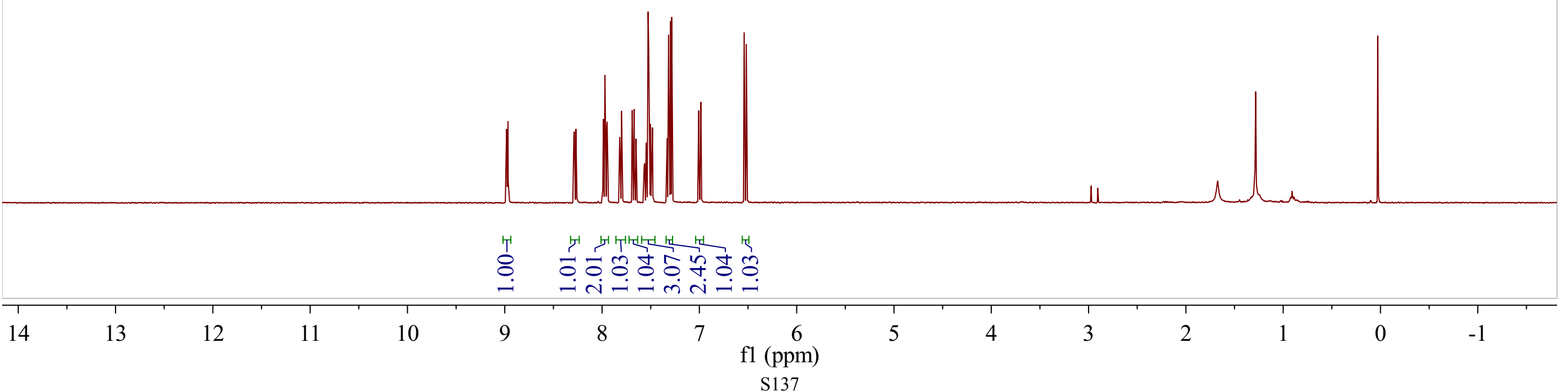




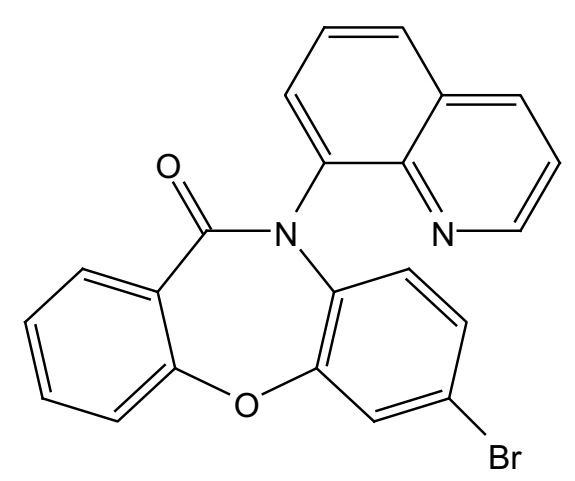

$8 \mathrm{k}$

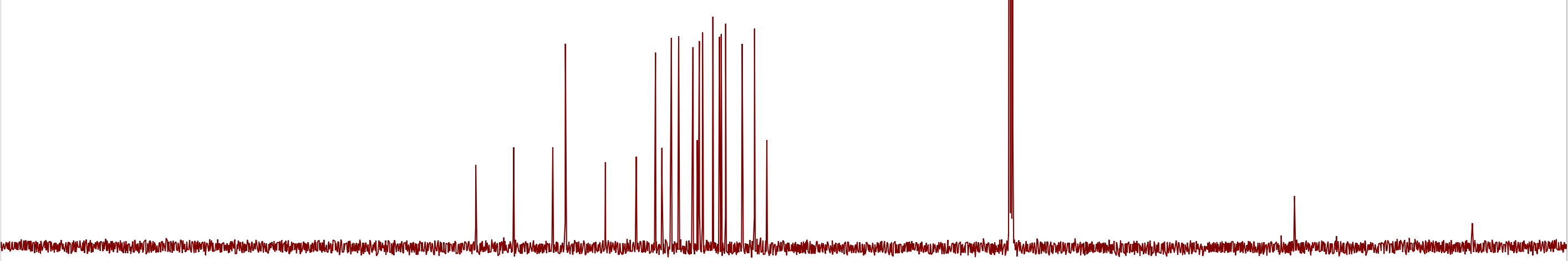

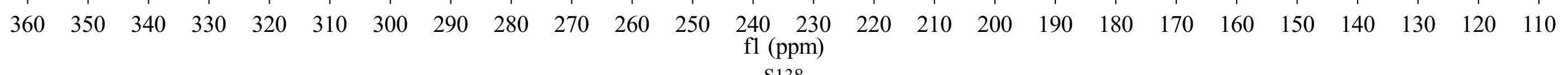




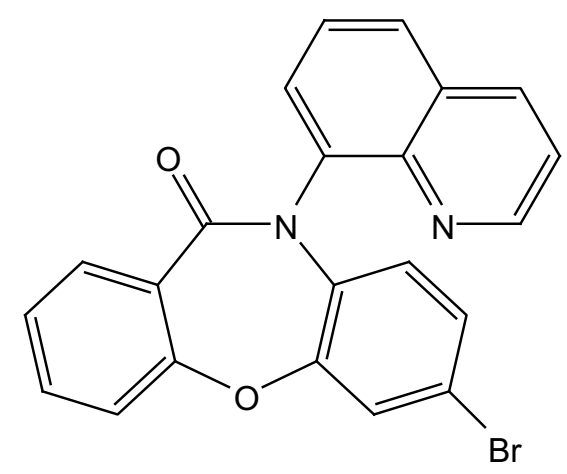

$8 k$

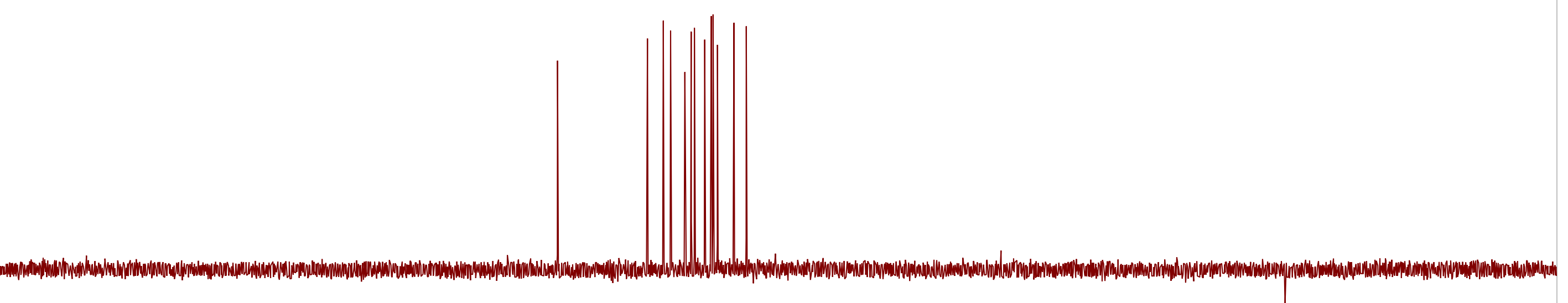

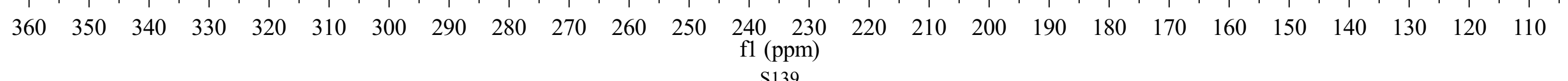


<smiles>COc1ccc2c(c1)Oc1ccc(Cl)cc1NC2=O</smiles>

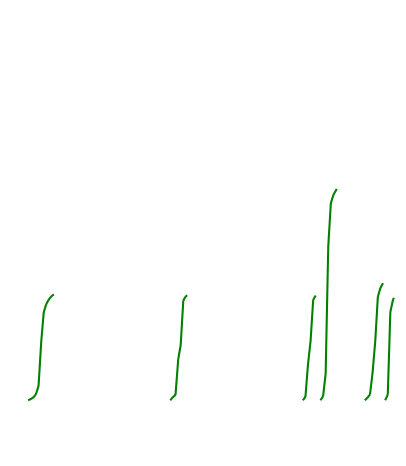

$9 a$

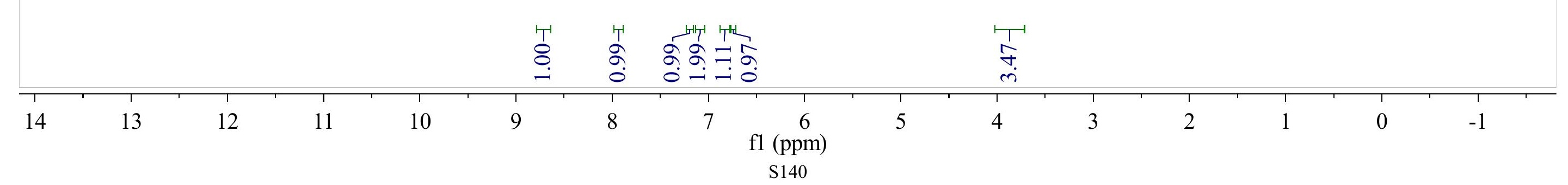




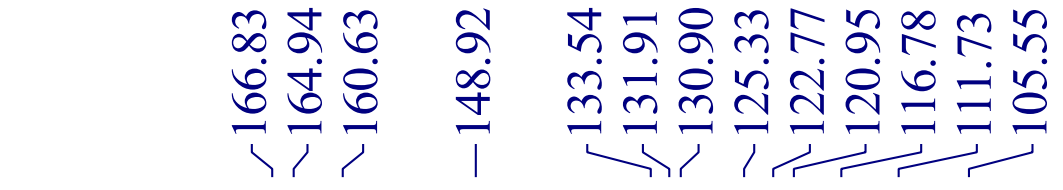

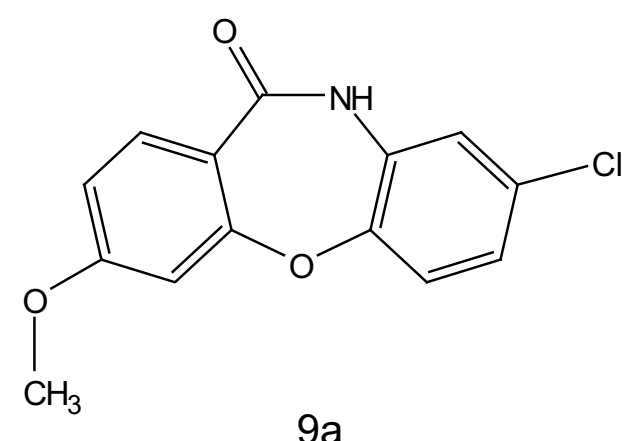

$9 a$

\begin{tabular}{|c|c|c|c|c|c|c|c|c|c|c|c|c|c|c|c|c|c|c|c|c|c|c|c|c|}
\hline 1 & 1 & 1 & 1 & 1 & 1 & 1 & 1 & 1 & 1 & 1 & 1 & 1 & 1 & $T$ & 1 & 1 & 1 & 1 & 1 & $T$ & 1 & $T$ & 1 & $a_{1}$ \\
\hline 230 & 220 & 210 & 200 & 190 & 180 & 170 & 160 & 150 & 140 & 130 & 120 & $\begin{array}{l}110 \\
(\mathrm{ppm}\end{array}$ & 100 & 90 & 80 & 70 & 60 & 50 & 40 & 30 & 20 & 10 & 0 & -10 \\
\hline
\end{tabular}


<smiles>COc1ccc2c(c1)Oc1ccc(Cl)cc1NC2=O</smiles>

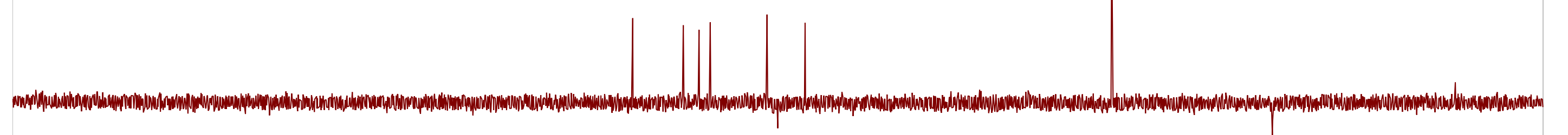

$\begin{array}{rlllllllllllllllllllllllll} & 230 & 220 & 210 & 200 & 190 & 180 & 170 & 160 & 150 & 140 & 130 & 120 & 110 & 100 & 90 & 80 & 70 & 60 & 50 & 40 & 30 & 20 & 10 & 0 & -10\end{array}$


<smiles>Cc1ccc2c(c1)Oc1ccc(Cl)cc1NC2=O</smiles>

$9 b$

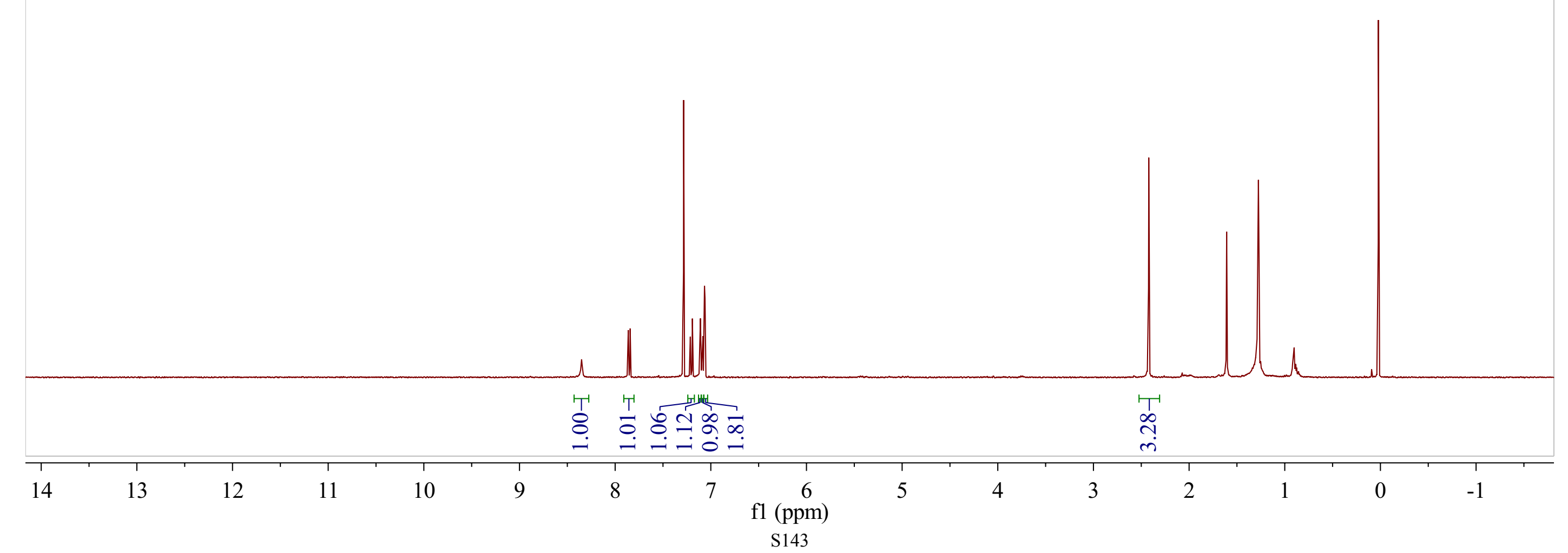


<smiles>Cc1ccc2c(c1)Oc1ccc(Cl)cc1NC2=O</smiles>

$9 b$

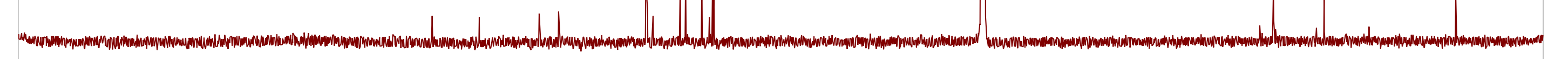

\begin{tabular}{|c|c|c|c|c|c|c|c|c|c|c|c|c|c|c|c|c|c|c|c|c|c|c|c|c|}
\hline 230 & 220 & 210 & 200 & 190 & 180 & 170 & 160 & 150 & 140 & 130 & 120 & 110 & 100 & 90 & 80 & 70 & 60 & 50 & 40 & 30 & 20 & 10 & 0 & -10 \\
\hline
\end{tabular}


<smiles>Cc1ccc2c(c1)Oc1ccc(Cl)cc1NC2=O</smiles>

$9 b$

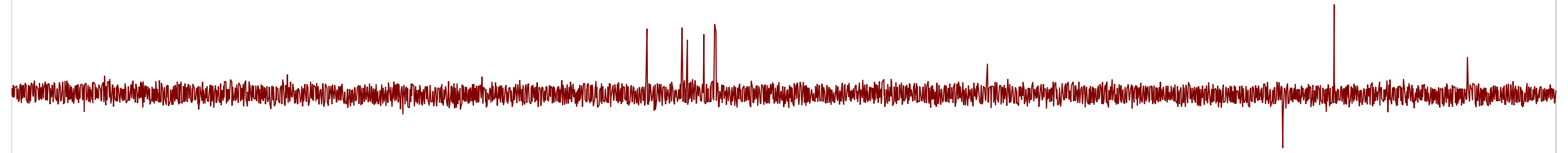

\begin{tabular}{|c|c|c|c|c|c|c|c|c|c|c|c|c|c|c|c|c|c|c|c|c|c|c|c|}
\hline 230 & 220 & 210 & 200 & 190 & 180 & 170 & 160 & 150 & 140 & 130 & 120 & fl 110100 & 90 & 80 & 70 & 60 & 50 & 40 & 30 & 20 & 10 & 0 & -10 \\
\hline
\end{tabular}



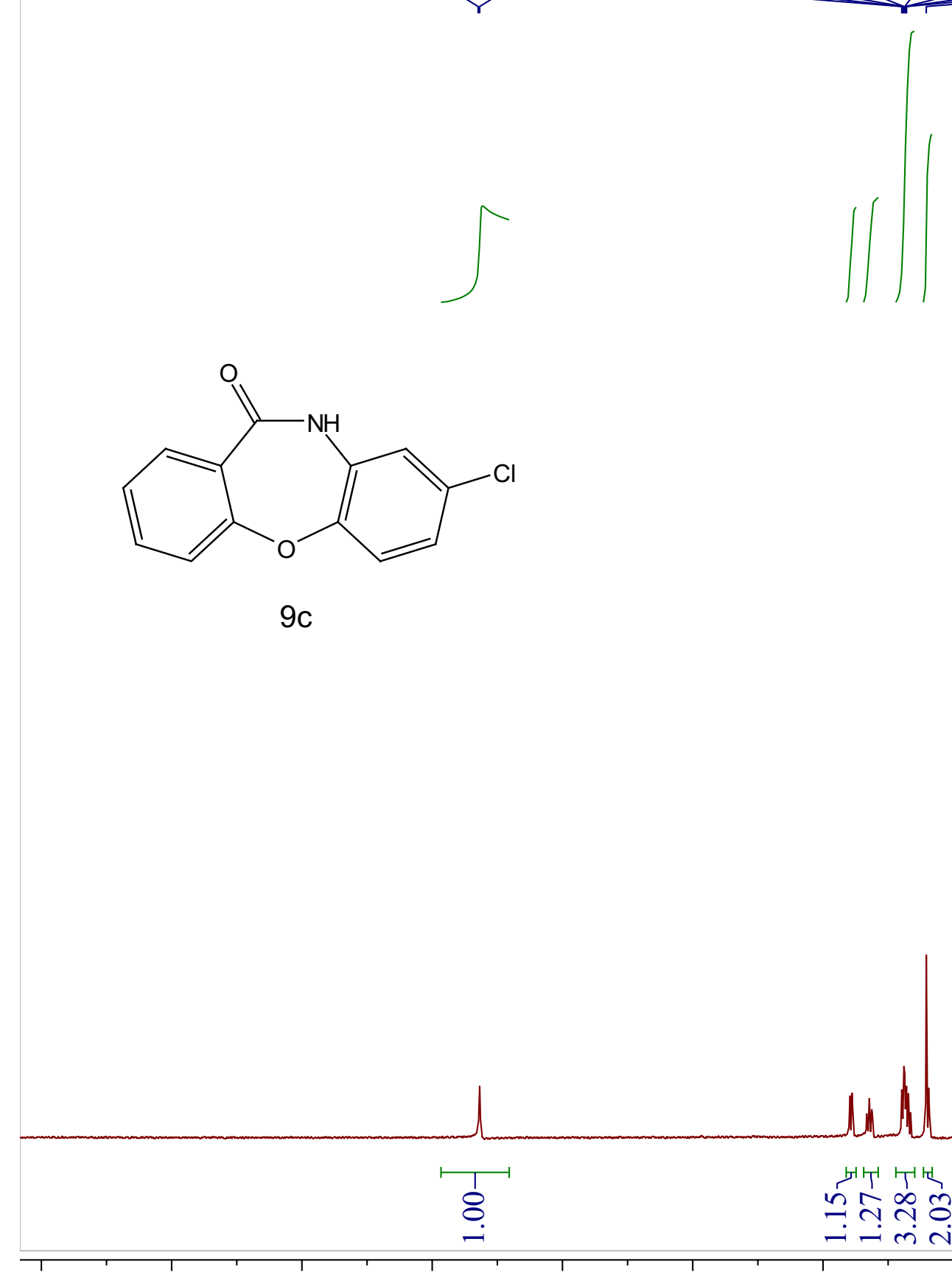


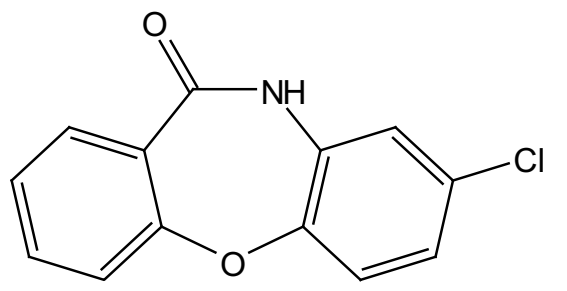

$9 c$

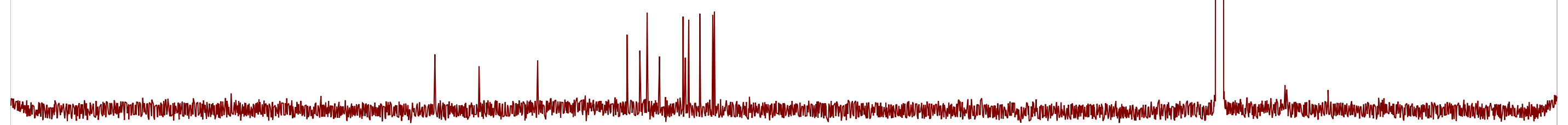

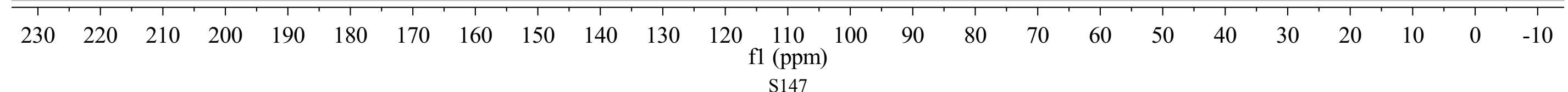




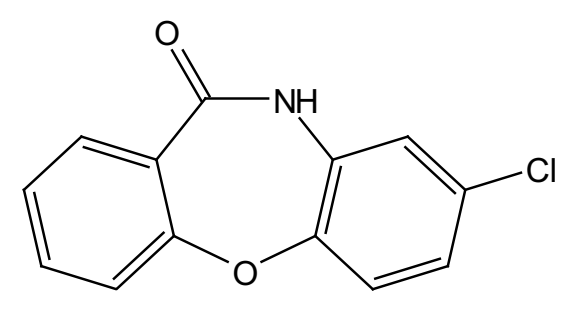

$9 c$

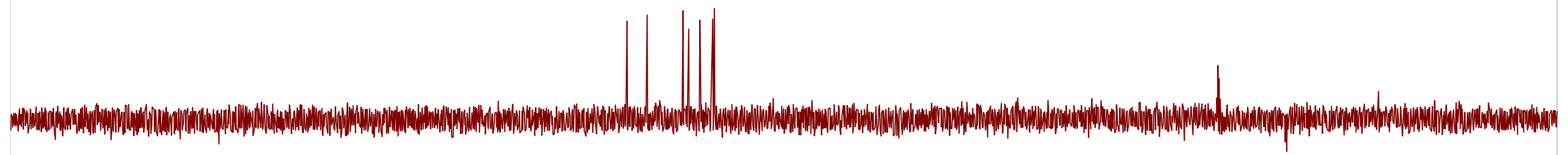

\begin{tabular}{llllllllllllllllllllllllll}
\hline 230 & 220 & 210 & 200 & 190 & 180 & 170 & 160 & 150 & 140 & 130 & 120 & $\begin{array}{c}110 \\
\mathrm{fl}(\mathrm{ppm})\end{array}$ & 100 & 90 & 80 & 70 & 60 & 50 & 40 & 30 & 20 & 10 & 0 & -10
\end{tabular}



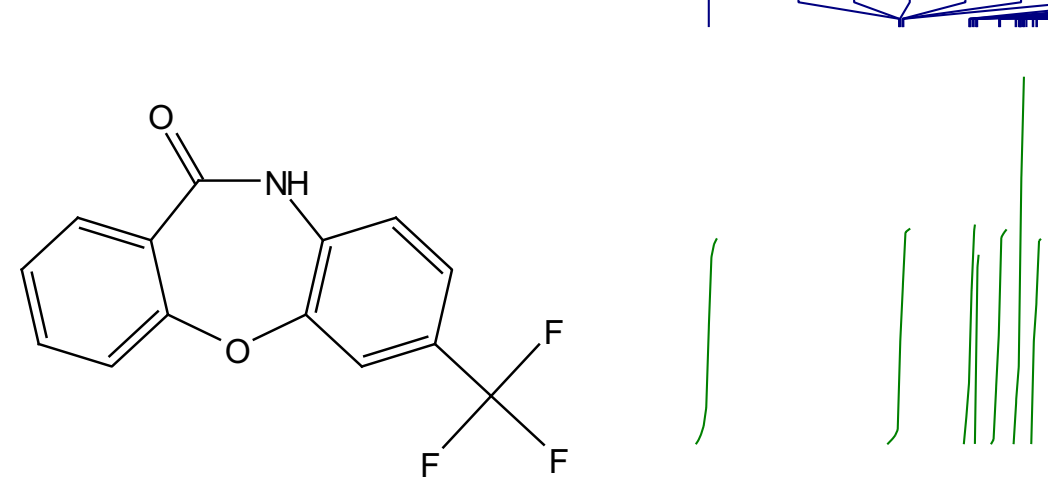

$9 d$

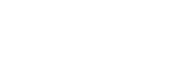

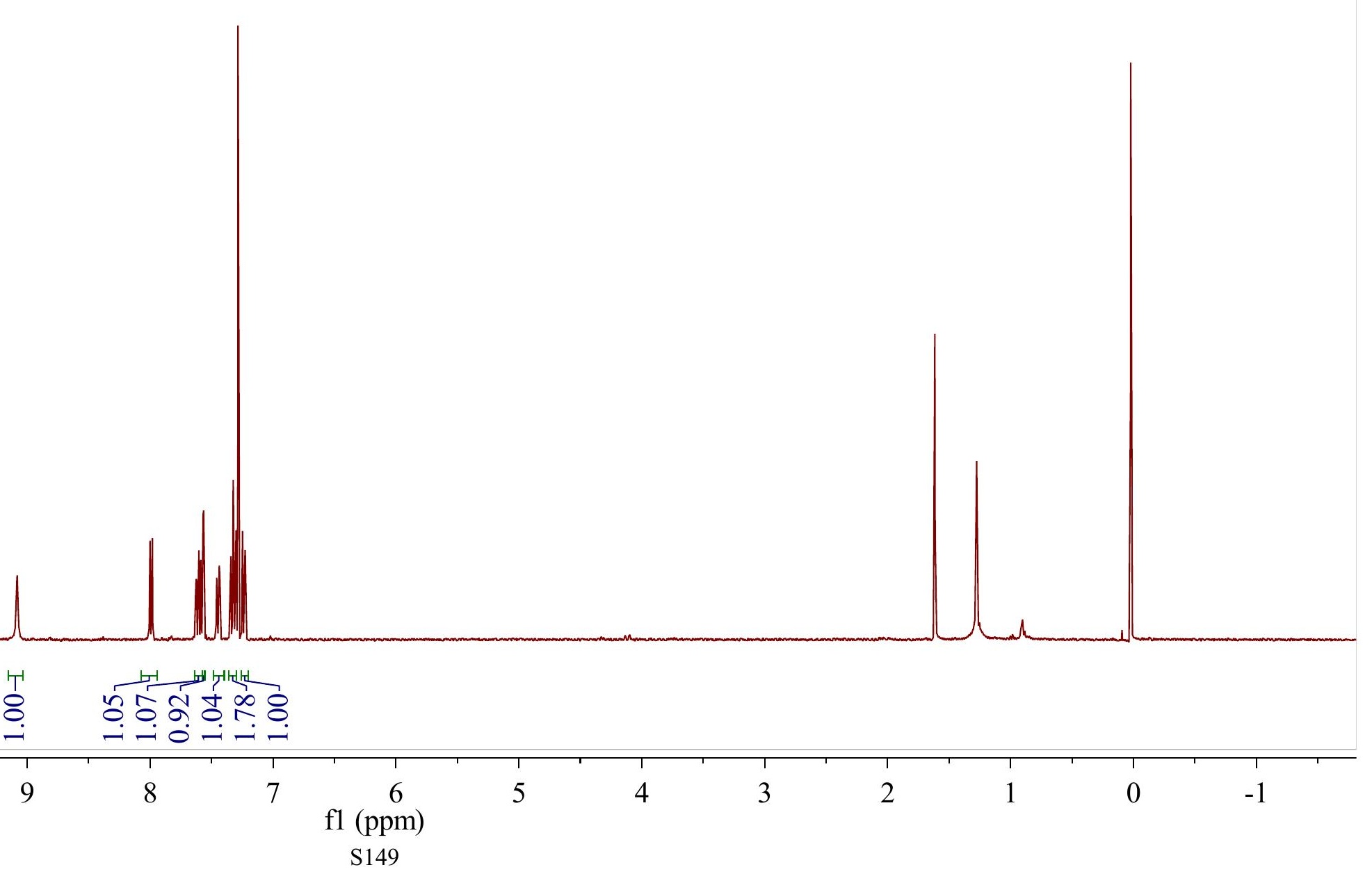




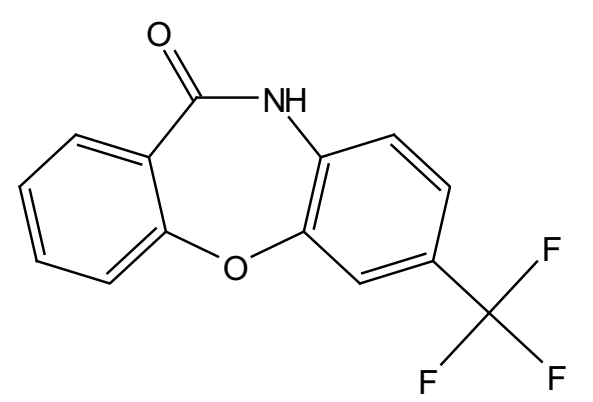

$9 d$

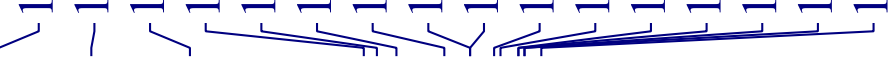

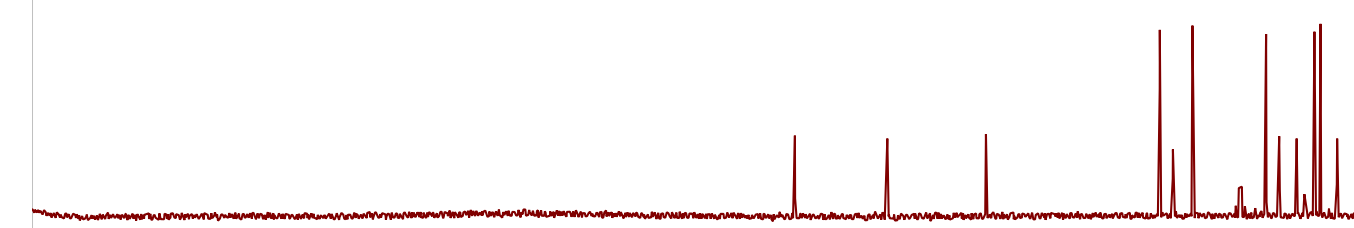

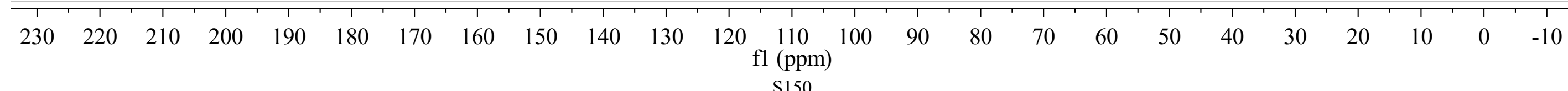




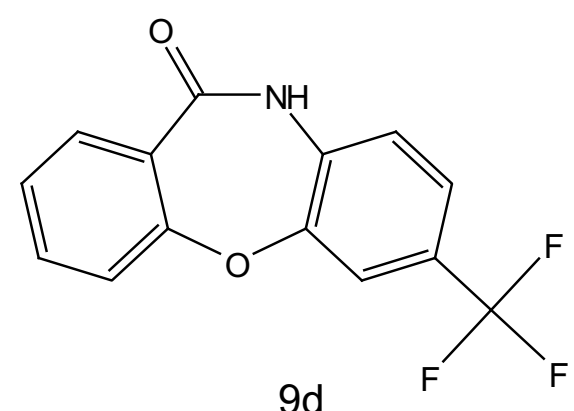

$9 d$

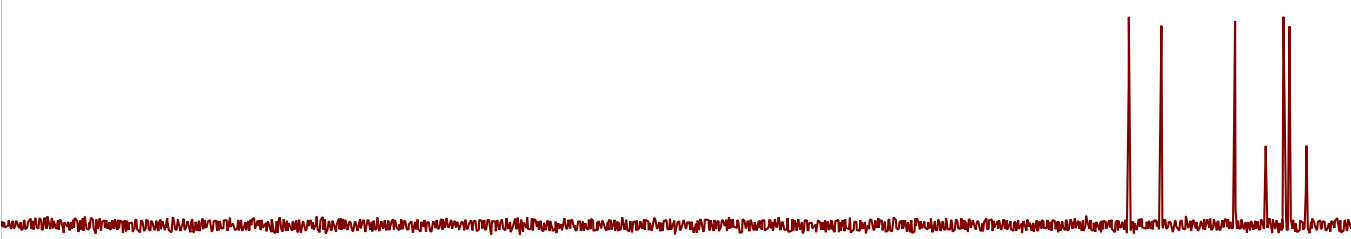

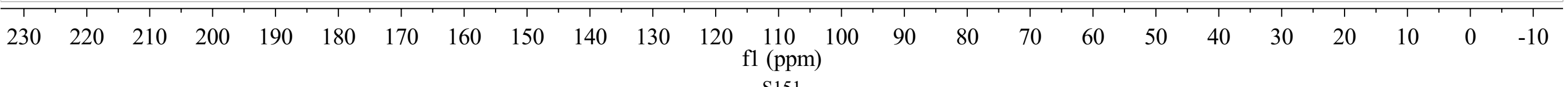



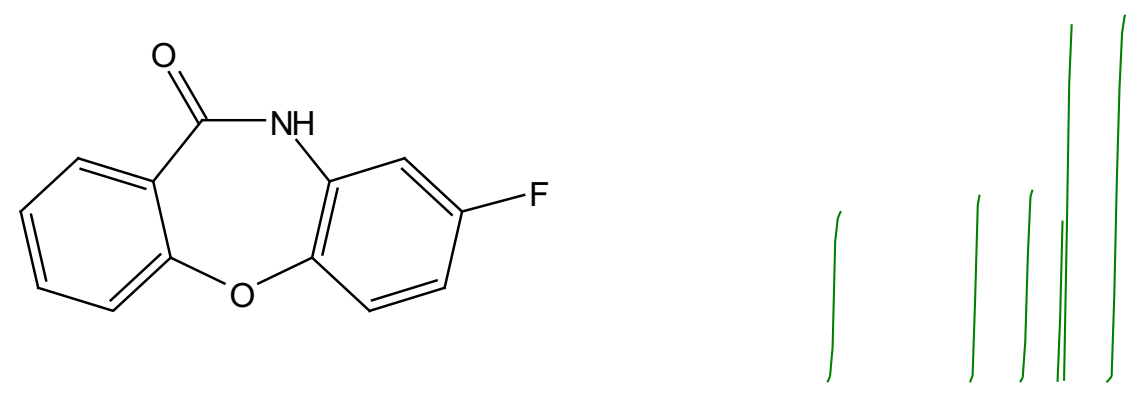

$9 e$

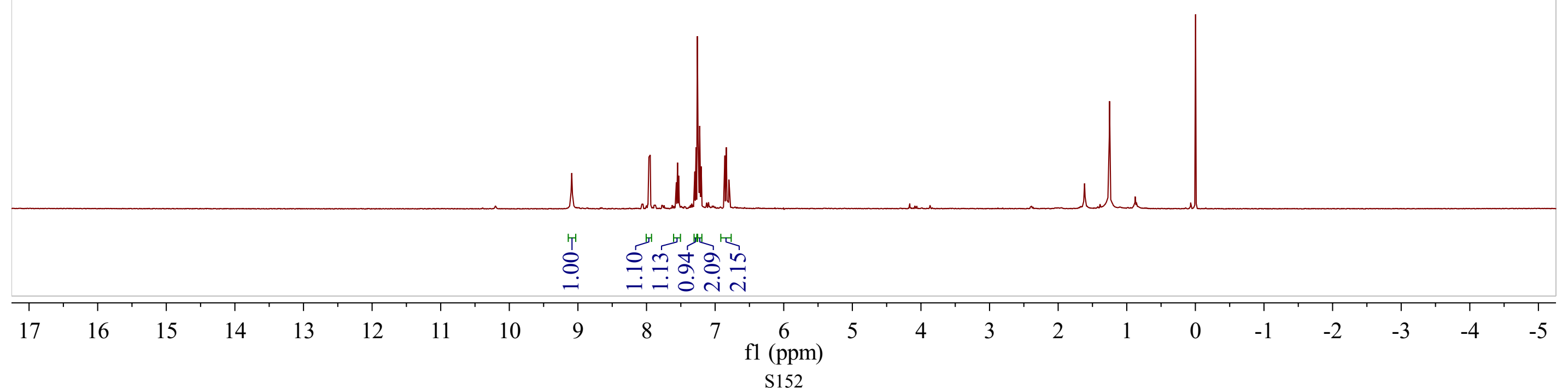




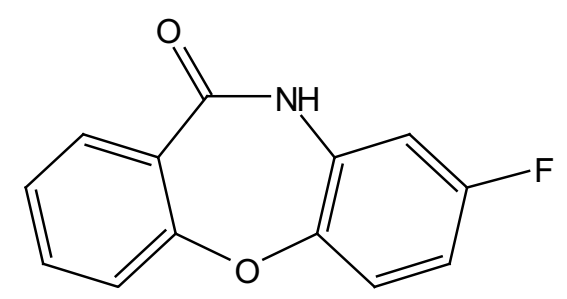

$9 e$

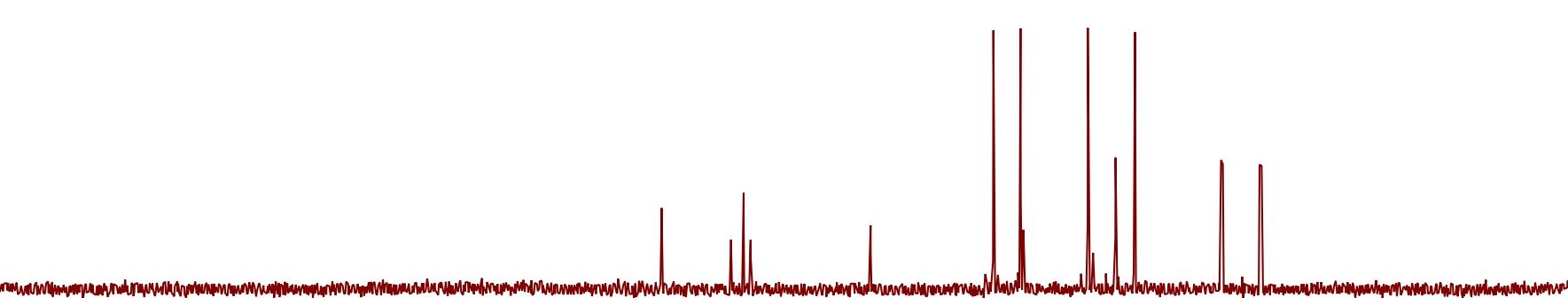

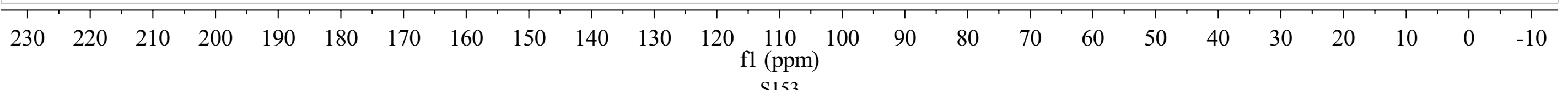




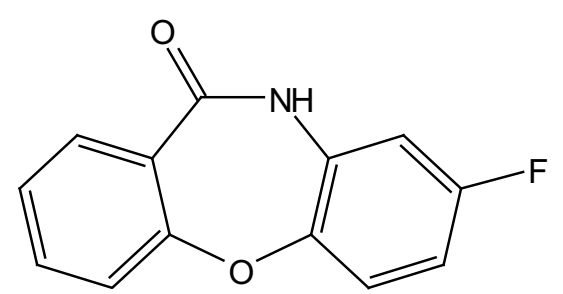

$9 e$

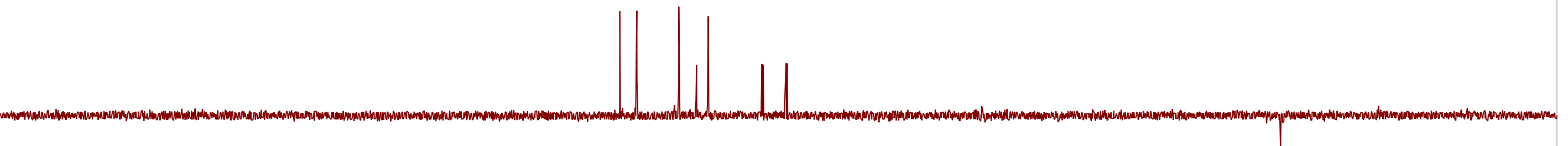

\begin{tabular}{lllllllllllllllllllllllllllll}
\hline & 230 & 220 & 210 & 200 & 190 & 180 & 170 & 160 & 150 & 140 & 130 & 120 & 110 & 100 & 90 & 80 & 70 & 60 & 50 & 40 & 30 & 20 & 10 & 0 & -10
\end{tabular}



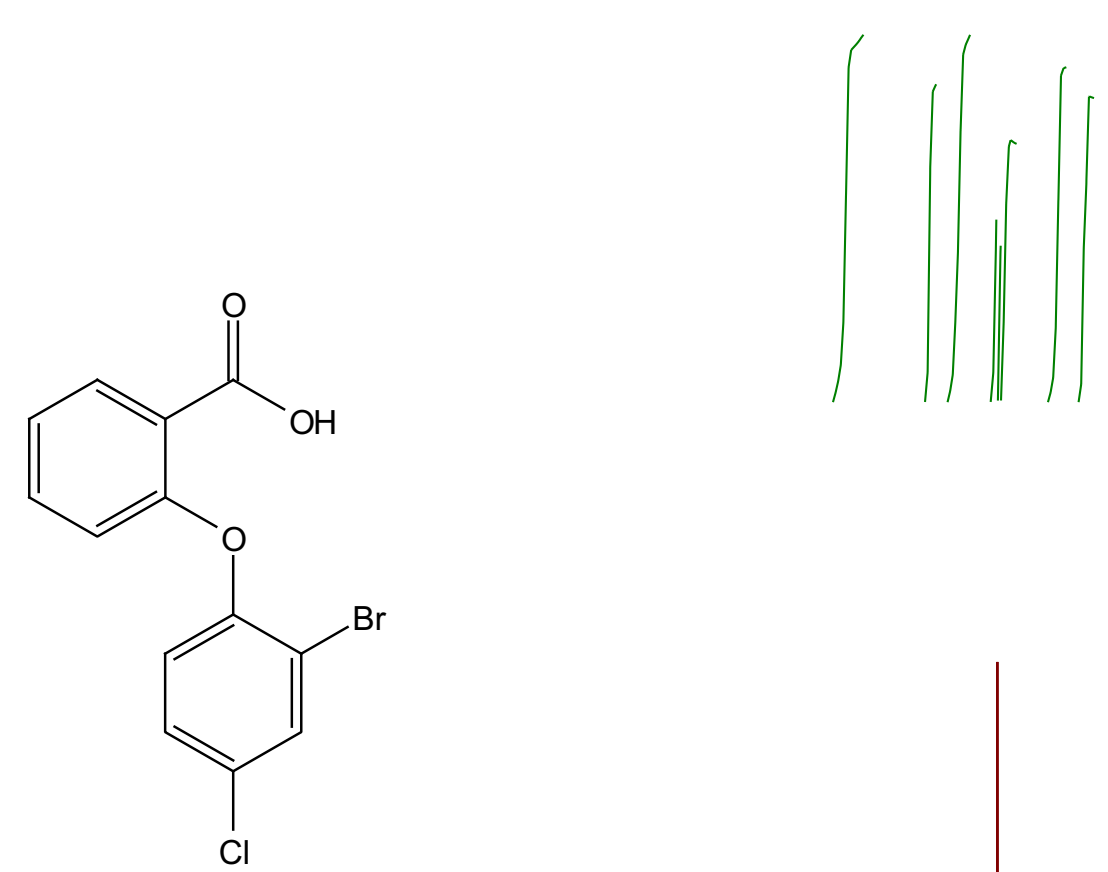

10

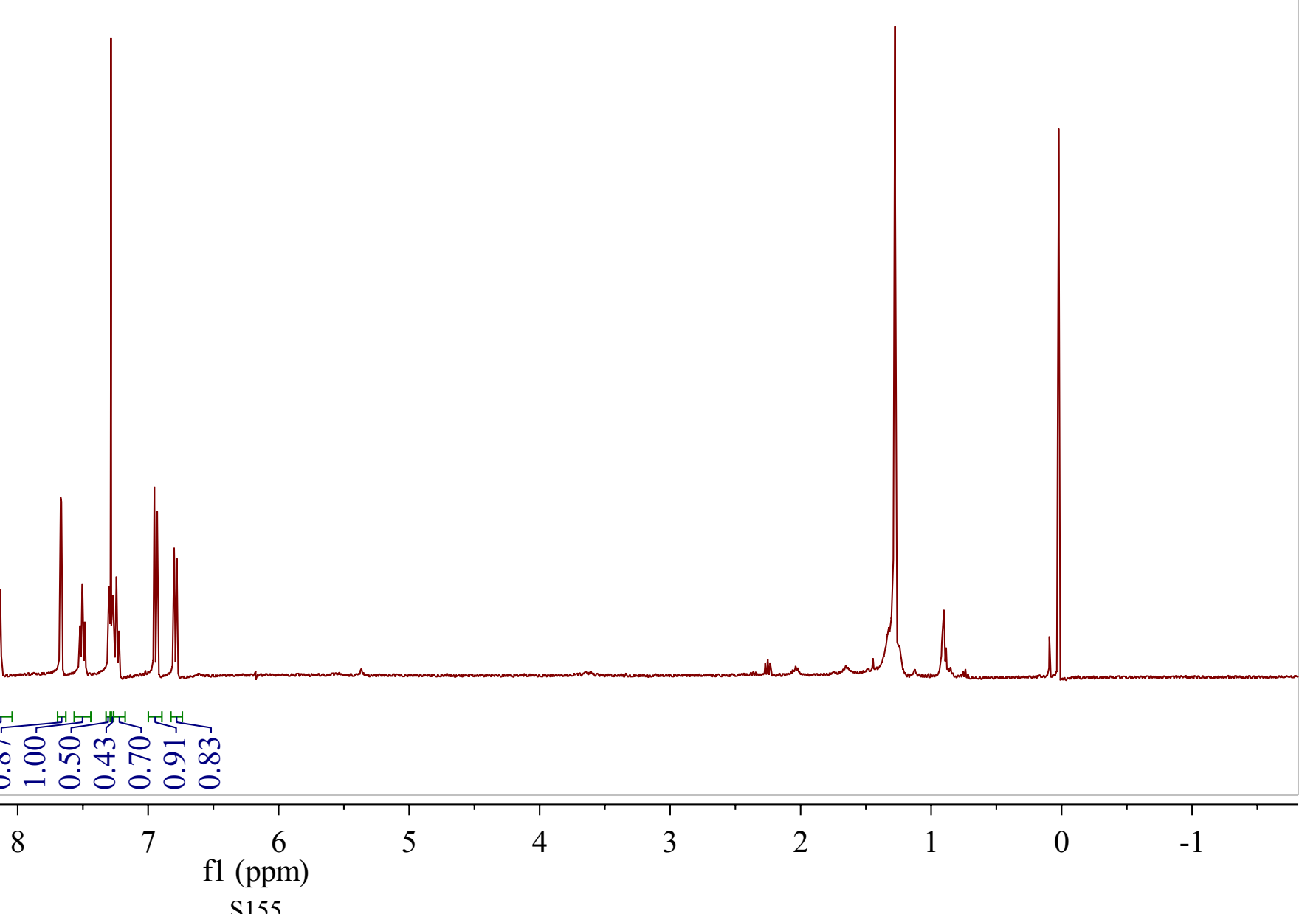



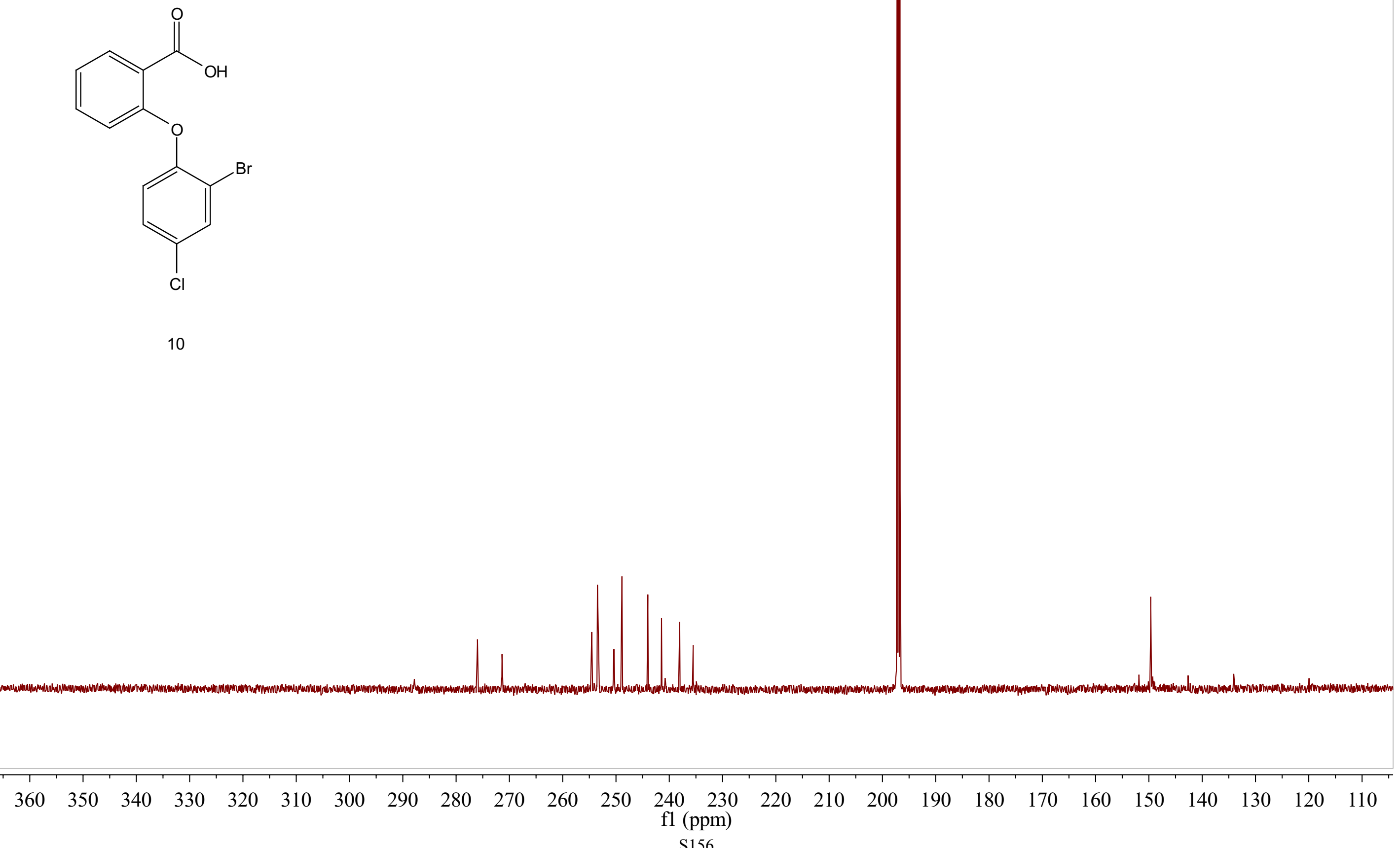


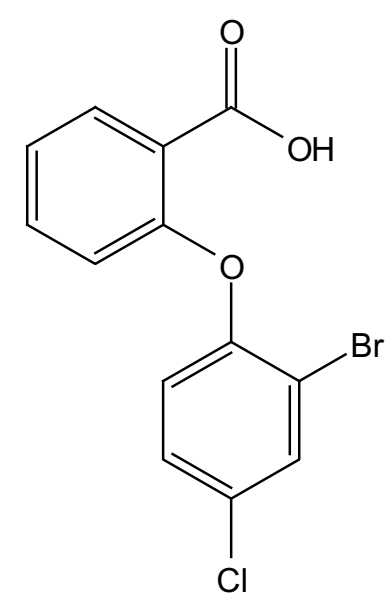

10

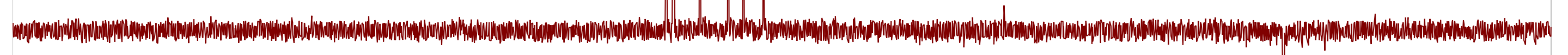

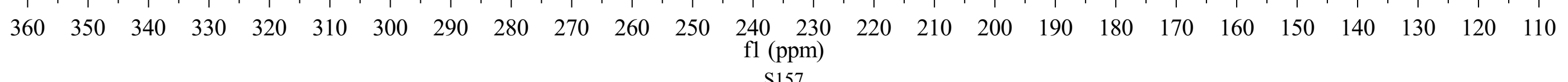




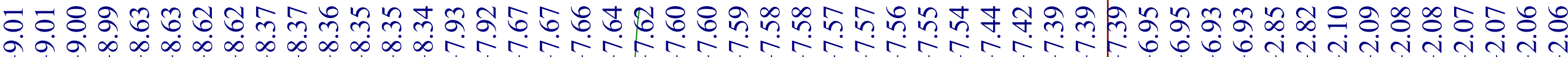

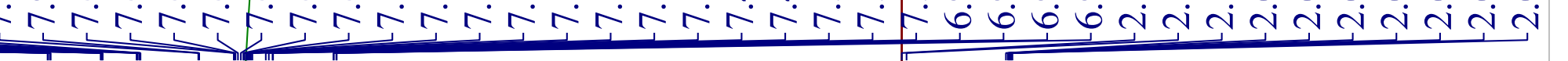
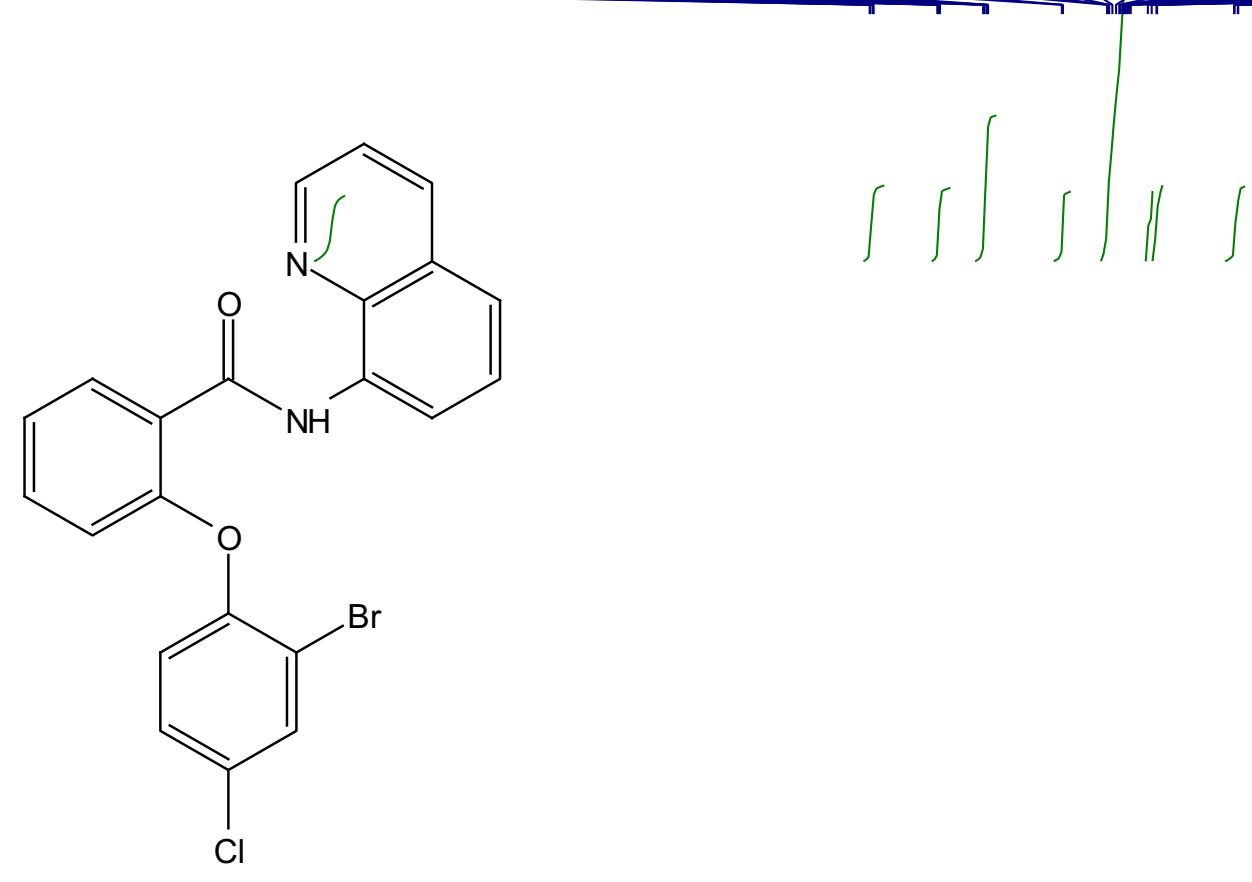

M1
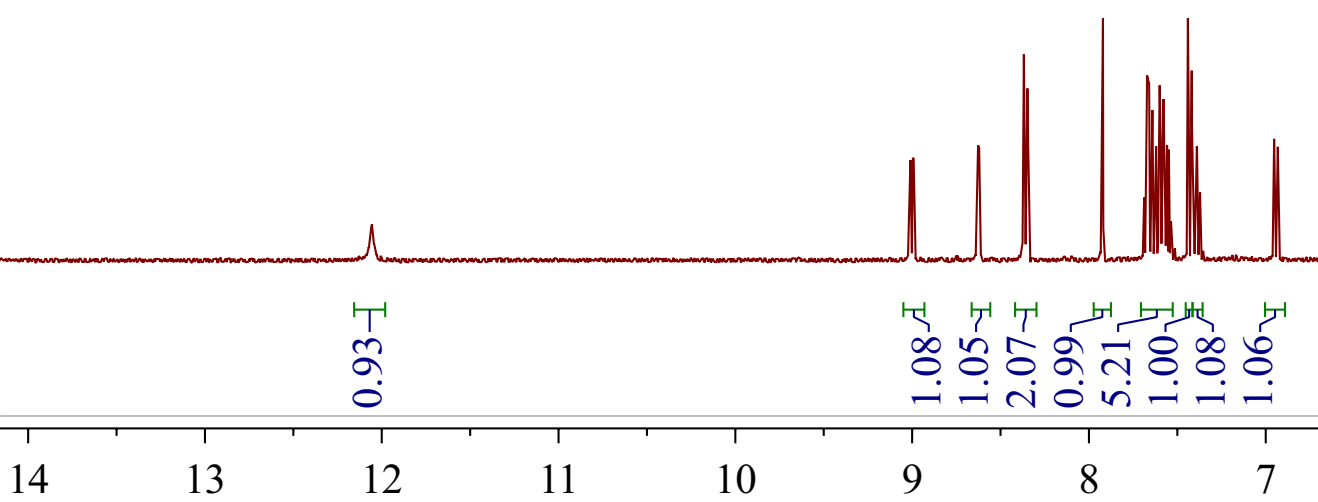

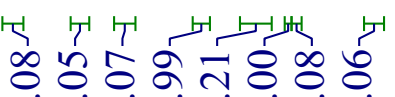
- - vi ñ 


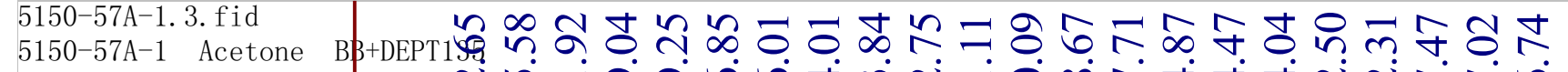

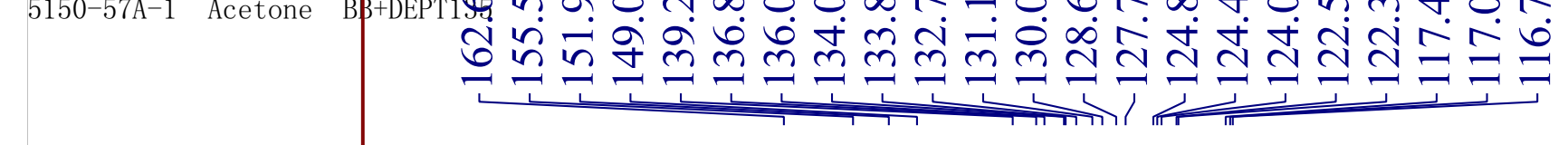

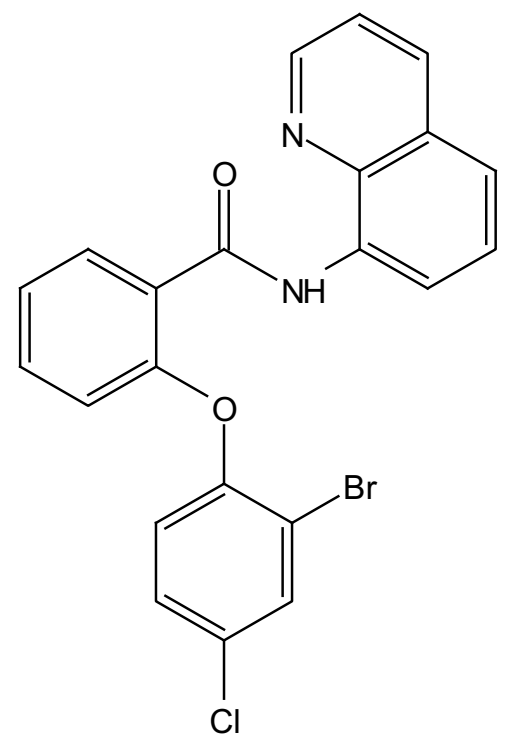

M1

$230 \quad 220 \quad 210$

200

$190 \quad 180$

170160

$150 \quad 140 \quad 130$

$120 \underset{\mathrm{fl}(\mathrm{ppm})}{110} 100$ S159 

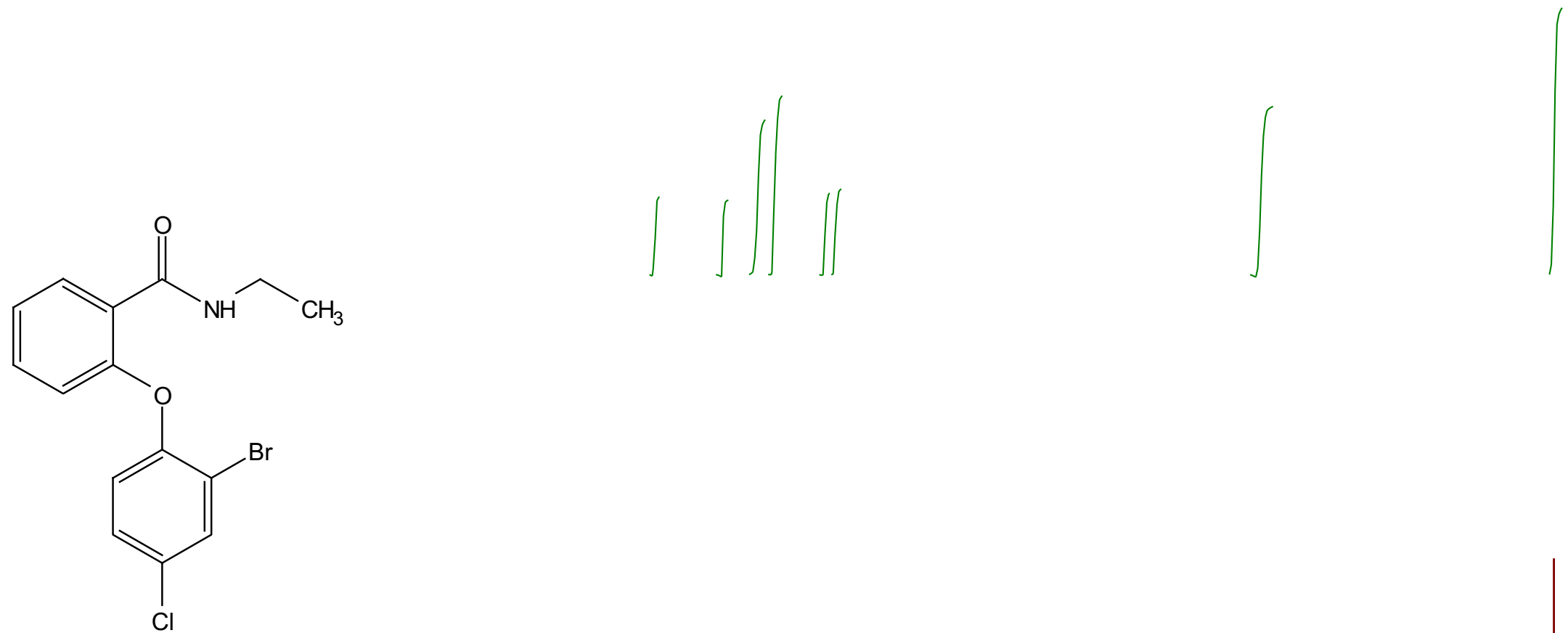

M2

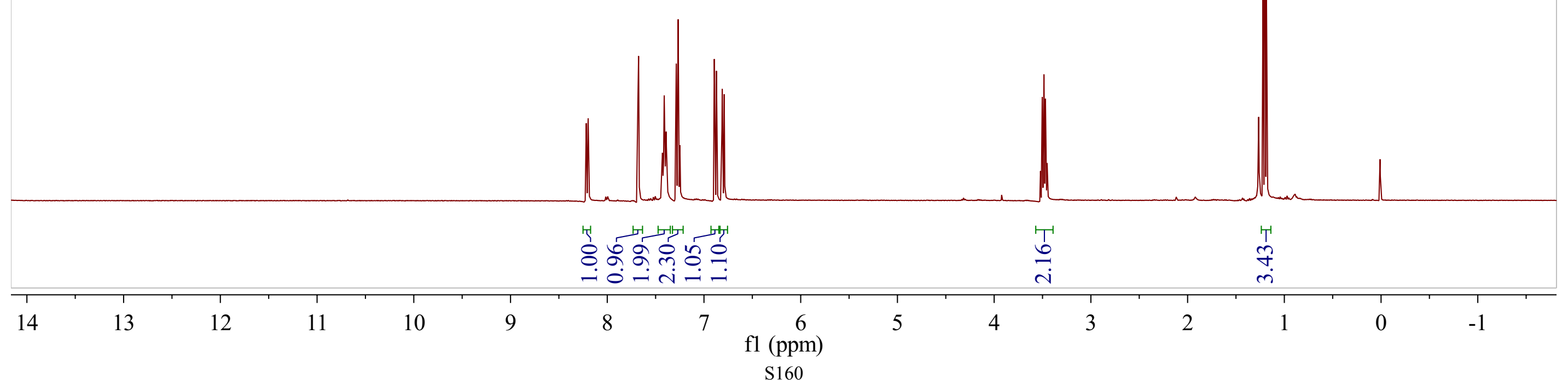




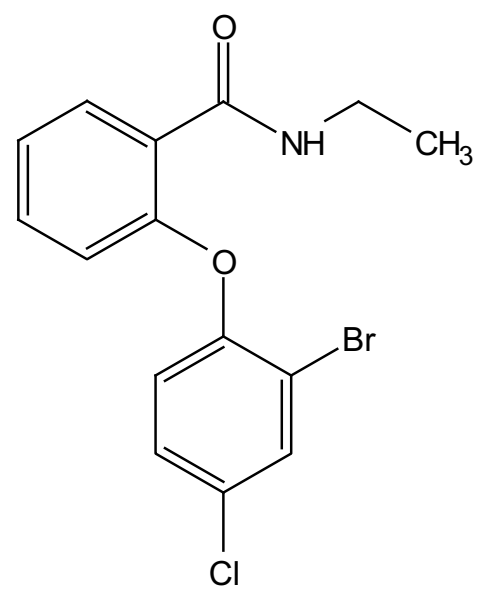

M2

\begin{tabular}{lllllllllllllllllllllllll}
\hline 230 & 220 & 210 & 200 & 190 & 180 & 170 & 160 & 150 & 140 & 130 & 120 & $\begin{array}{l}110 \\
\mathrm{fl}(\mathrm{ppm})\end{array}$ & 100 & 90 & 80 & 70 & 60 & 50 & 40 & 30 & 20 & 10 & 0 & -10
\end{tabular}




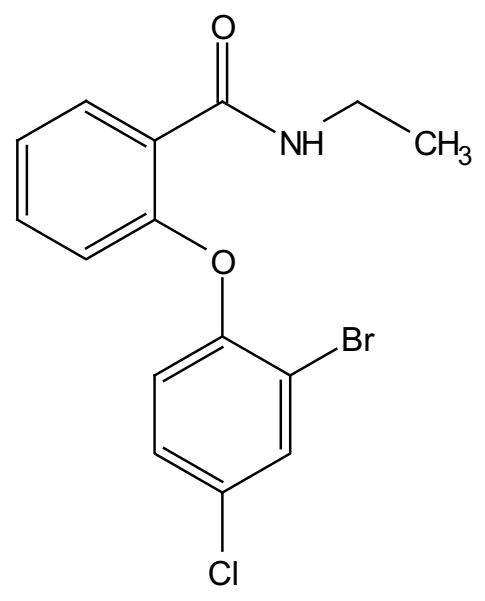

M2

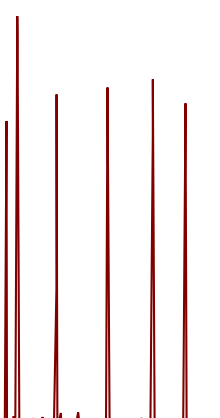

$\begin{array}{lllllllllllllllllllllllllllllllllll}230 & 220 & 210 & 200 & 190 & 180 & 170 & 160 & 150 & 140 & 130 & 120 & \begin{array}{l}110 \\ \mathrm{fl}(\mathrm{ppm})\end{array} & 100 & 90 & 80 & 70 & 60 & 50 & 40 & 30 & 20 & 10 & 0 & -10\end{array}$



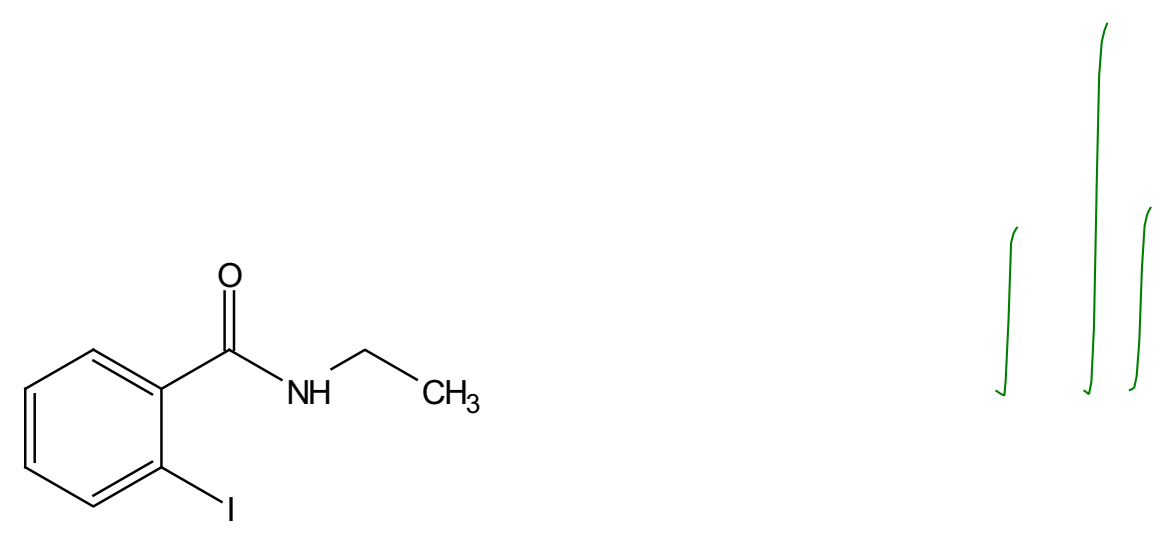

3

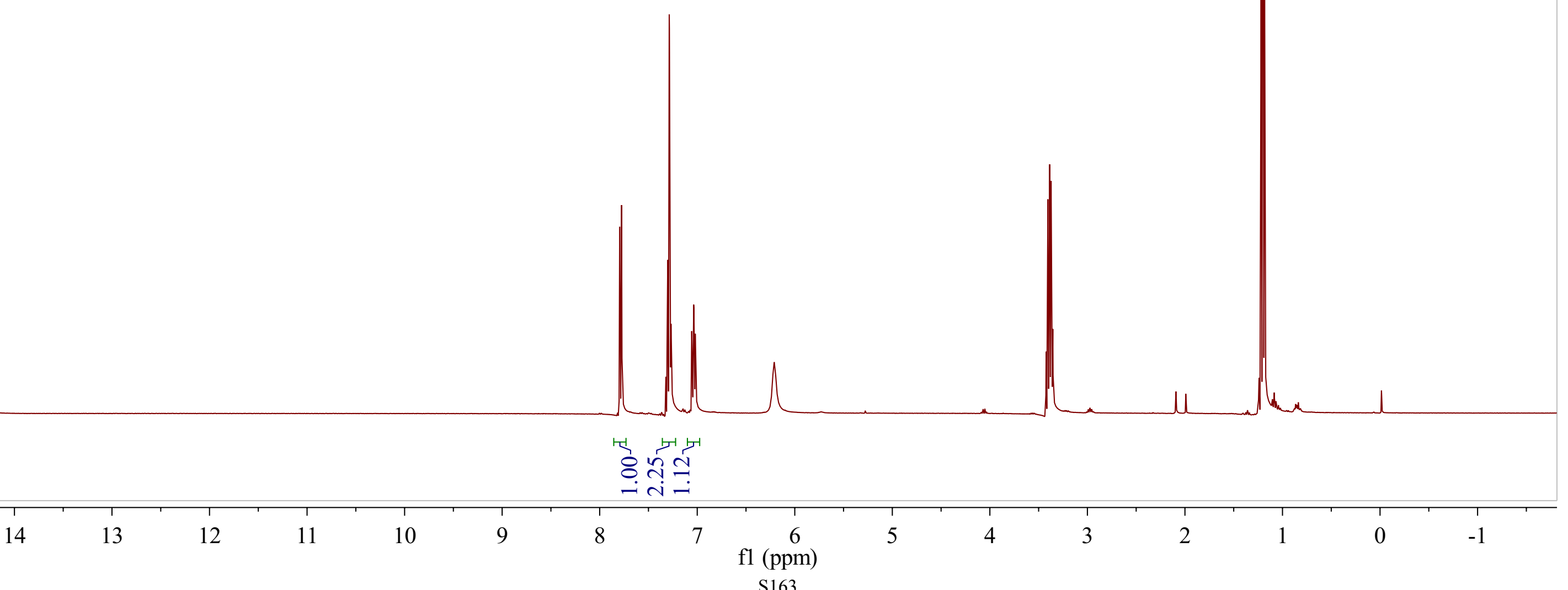



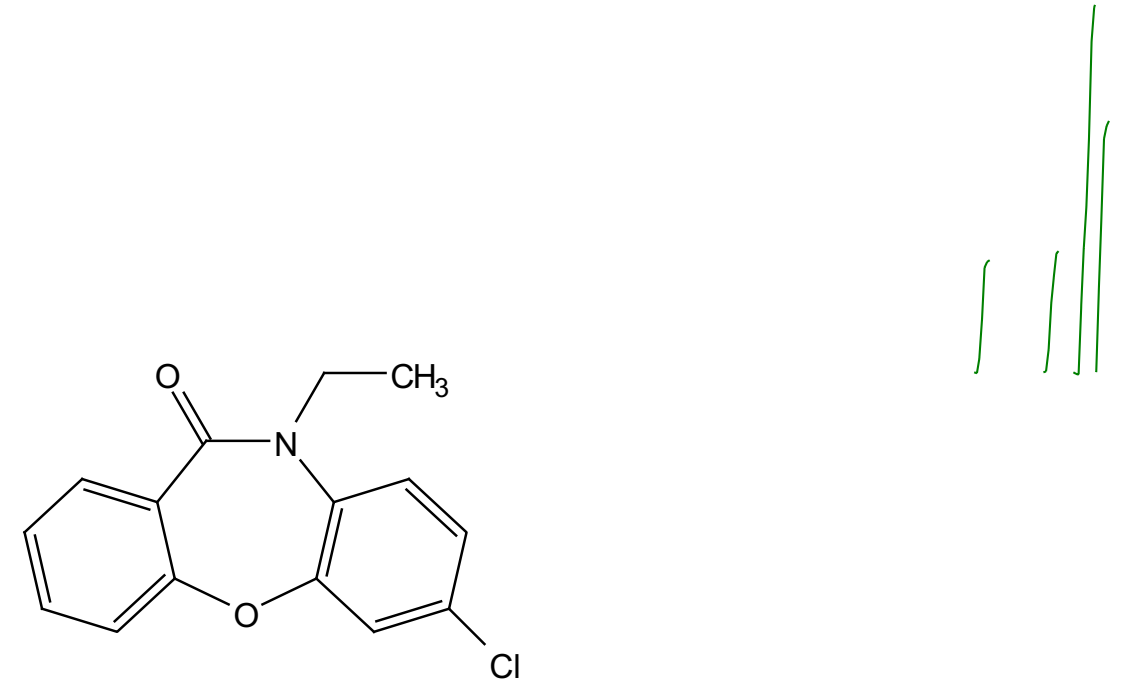

4

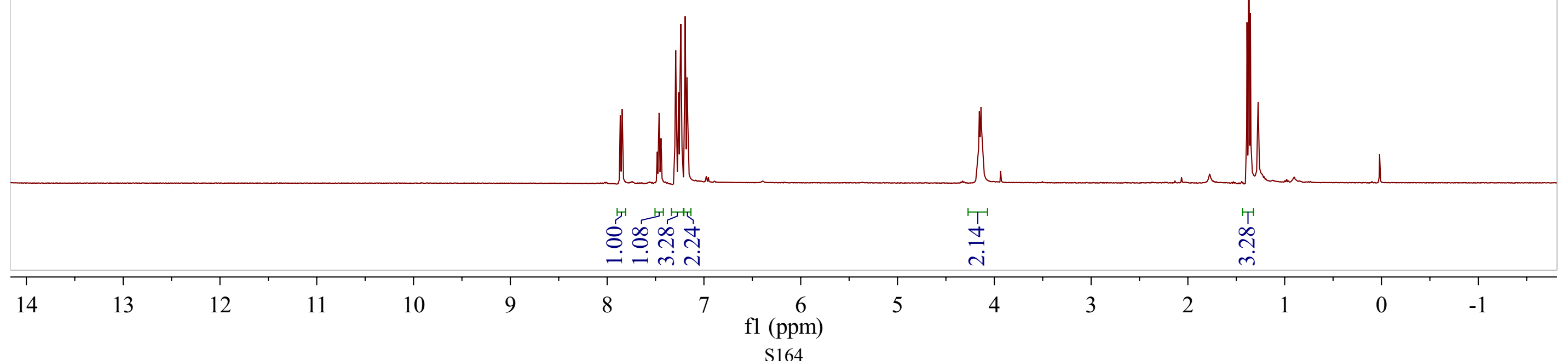




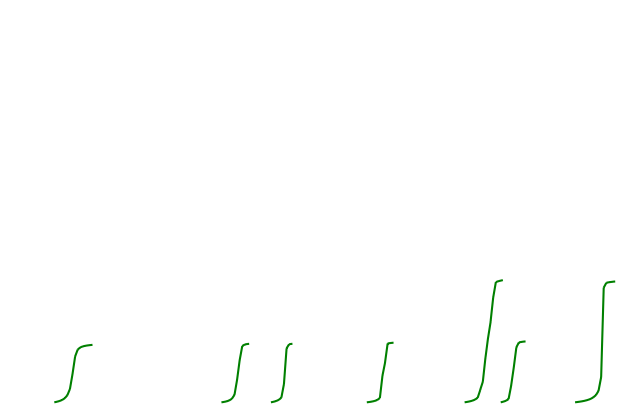

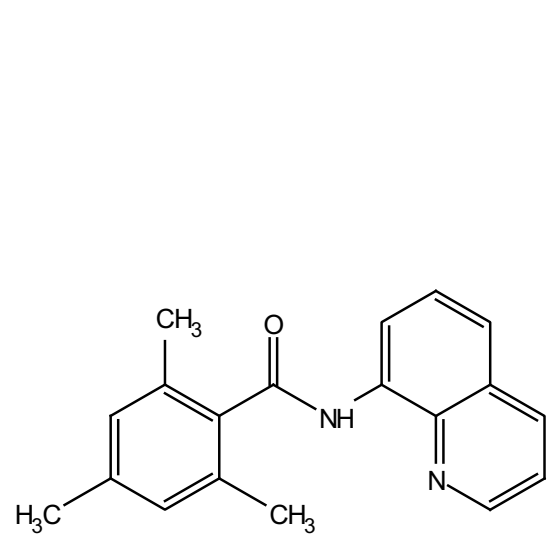

11

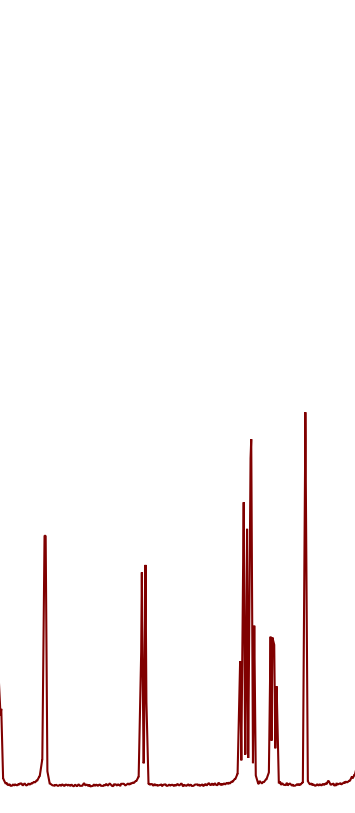

\begin{tabular}{|c|c|c|c|c|}
\hline 'T' & 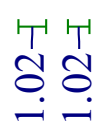 & 当 & 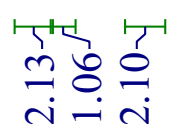 & 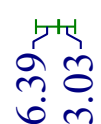 \\
\hline
\end{tabular}

13

12

10 fl (ppm)

S165 